$$
\begin{gathered}
\text { Aus dem Fachbereich Medizin } \\
\text { der Johann Wolfgang Goethe-Universität } \\
\text { Frankfurt am Main }
\end{gathered}
$$

betreut am

Zentrum der Chirurgie

Klinik für Mund-, Kiefer- und Plastische Gesichtschirurgie

Direktor: Prof. Dr. Dr. Dr. Robert Sader

\title{
Evaluation verschiedener Lehrformate in der Vermittlung allgemeinmedizinischer Basiskompetenzen innerhalb einer studentisch betriebenen Poliklinik
}

\author{
Dissertation \\ zur Erlangung des Doktorgrades der Medizin \\ des Fachbereichs Medizin \\ der Johann Wolfgang Goethe-Universität \\ Frankfurt am Main
}

vorgelegt von

Arda Manap

aus Berlin

Frankfurt am Main, 2020 
Dekan:

Referent/in:

Korreferent/in:

Tag der mündlichen Prüfung:
Prof. Dr. Stefan Zeuzem

Prof. Dr. Dr. Dr. Robert Sader

Prof. Dr. Miriam Rüsseler

03.05.2021 
Meiner Familie gewidmet 


\section{INHALTSVERZEICHNIS}

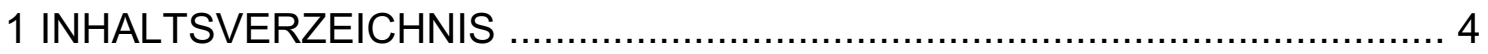

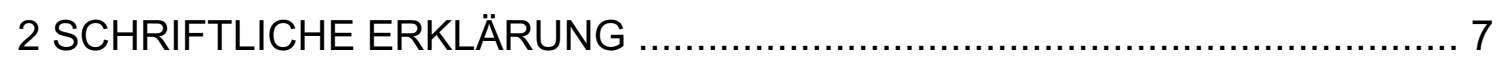

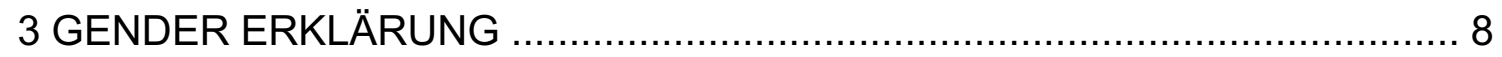

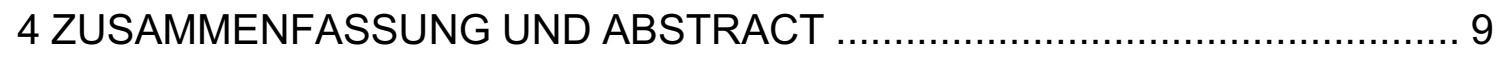

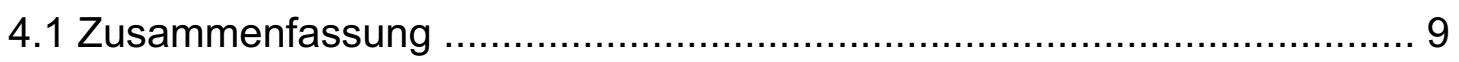

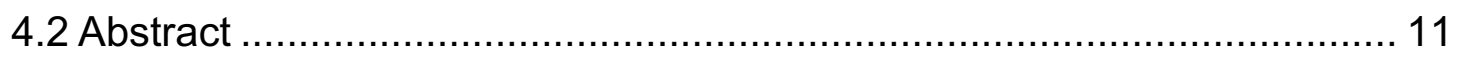

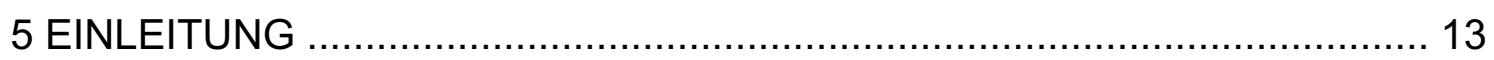

5.1 Gründung und Anspruch der Studentischen Poliklinik Frankfurt ............. 13

5.2 Konzeption der Student-run Free Clinics ................................................ 16

5.3 Peer-assisted Learning in der Medizin ................................................. 19

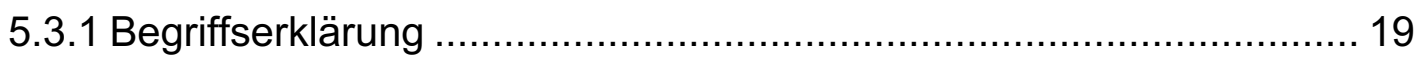

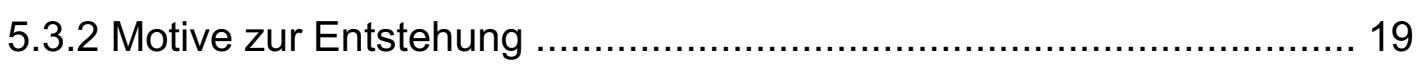

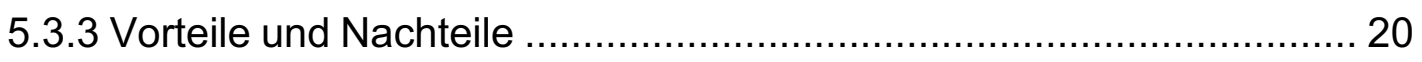

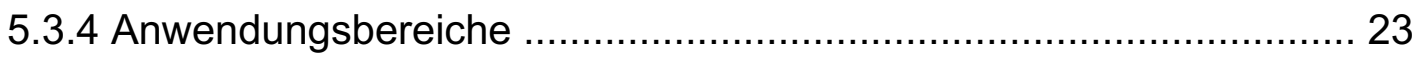

5.4 Virtual Patient Learning in der Medizin ................................................ 23

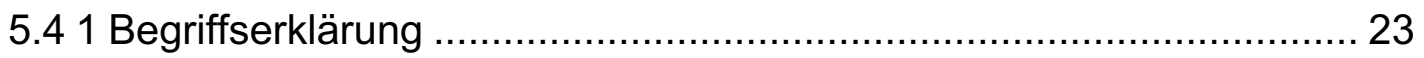

5.4.2 Motive zur Entstehung ………................................................. 25

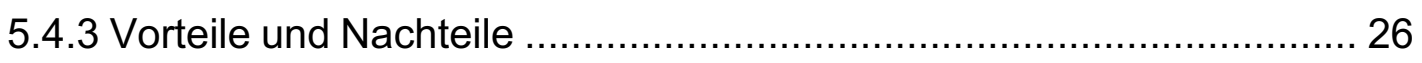

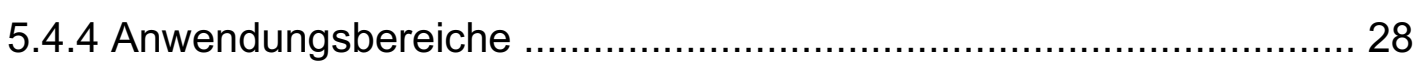

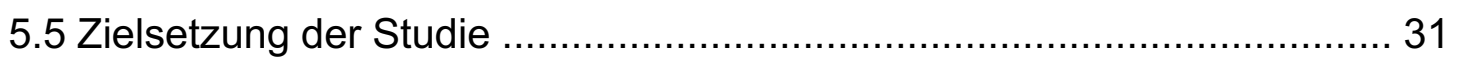

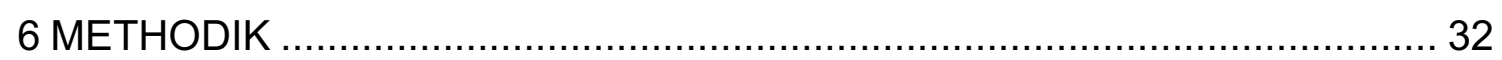

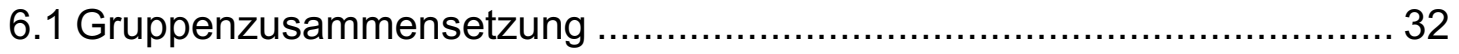

6.2 Aufbau des Wahlfachs der Studentischen Poliklinik Frankfurt ................. 33

6.2.1 Aufbau des Peer-assisted Learning Seminars ................................. 35

6.2.2 Aufbau des Virtual Patient Learning Seminars .................................. 38

6.2.3 Aufbau der Sprechstunde ........................................................... 41

6.2.4 Aufbau des curricularen Unterrichts ............................................. 42

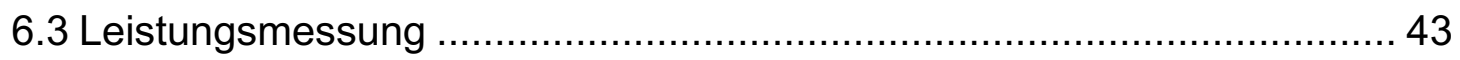

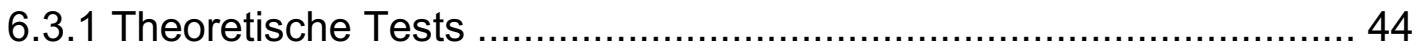

6.3.1.1 Theoretischer Langzeit-Test ................................................ 44

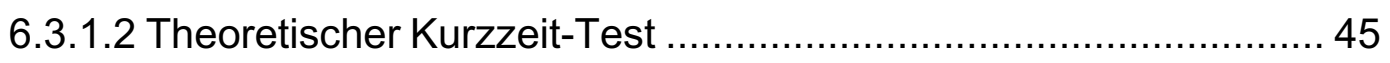

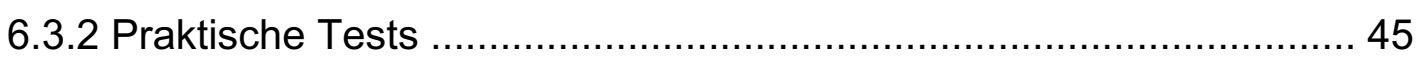




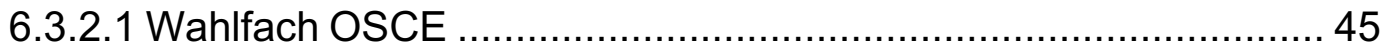

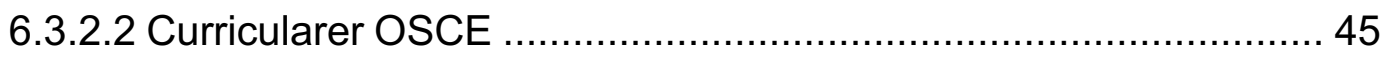

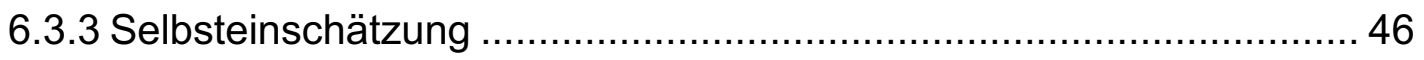

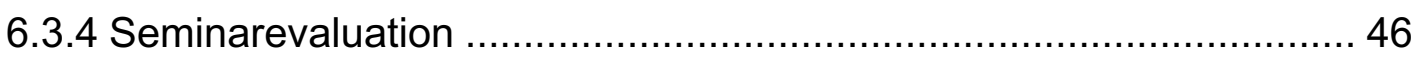

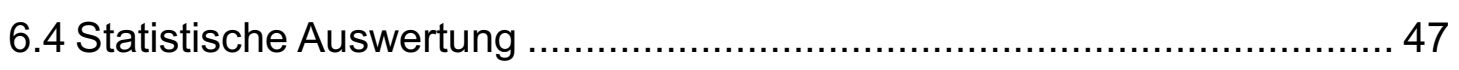

6.4.1 Auswertung der theoretischen Tests ........................................... 48

6.4.1.1 Auswertung des theoretischen Langzeit-Tests .......................... 48

6.4.1.2 Auswertung des theoretischen Kurzzeit-Tests ........................... 48

6.4.2 Auswertung der praktischen Tests ............................................... 49

6.4.2.1 Auswertung des Wahlfach OSCEs ........................................... 49

6.4.2.2 Auswertung des curricularen OSCEs ........................................ 49

6.4.3 Auswertung der Selbsteinschätzung ............................................. 49

6.4.4 Auswertung der Seminarevaluation .............................................. 49

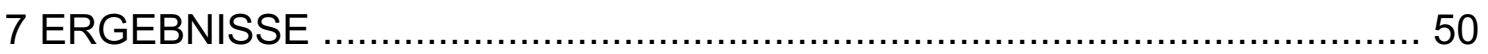

7.1 Demographische Daten der Studienteilnehmer.................................... 50

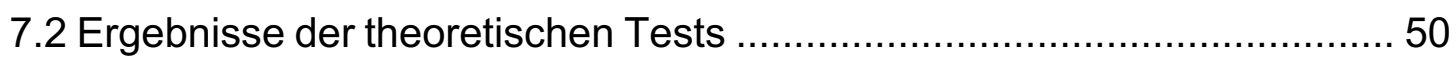

7.2.1 Ergebnisse des theoretischen Langzeit-Tests ............................... 50

7.2.2 Ergebnisse des theoretischen Kurzzeit-Tests .................................. 52

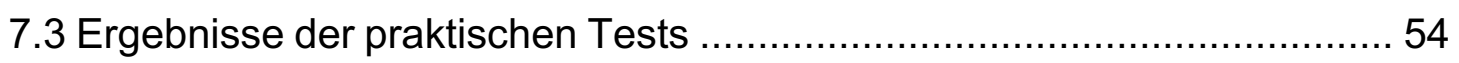

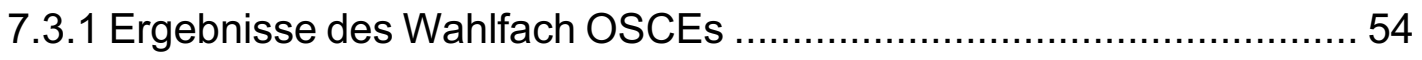

7.3.2 Ergebnisse des curricularen OSCEs ............................................. 54

7.4 Ergebnisse der Selbsteinschätzung ………................................... 55

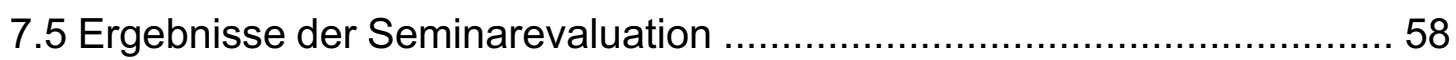

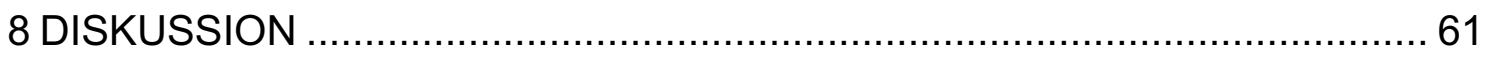

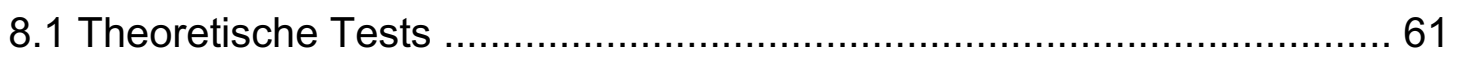

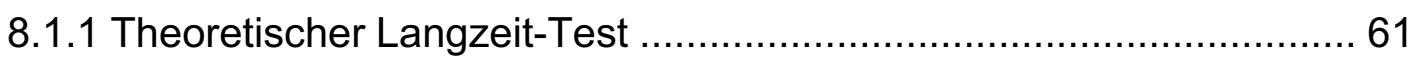

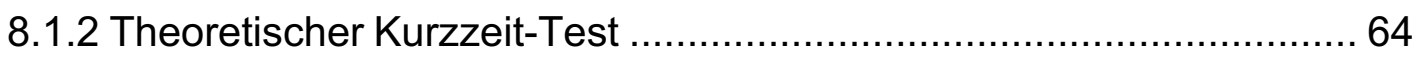

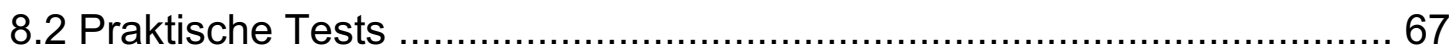

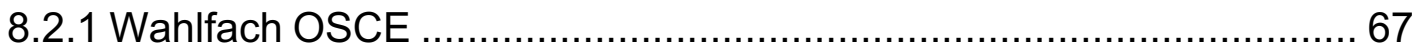

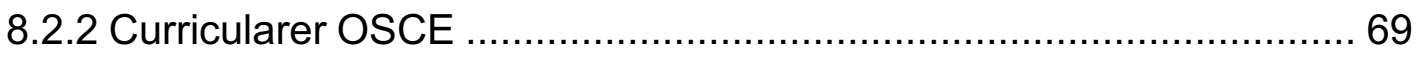

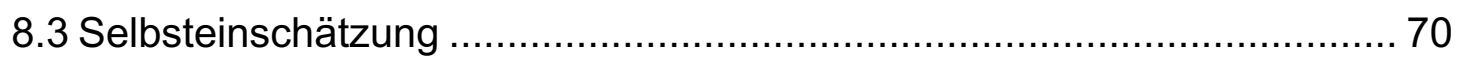

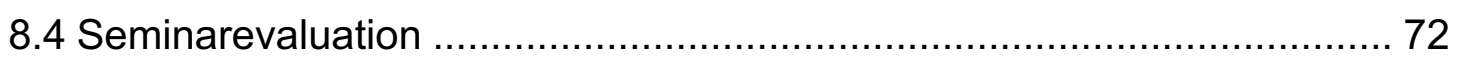

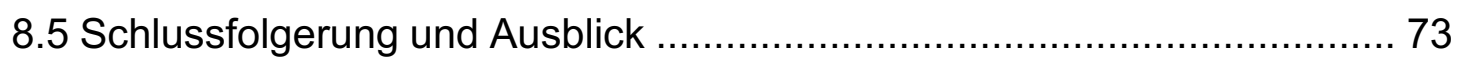

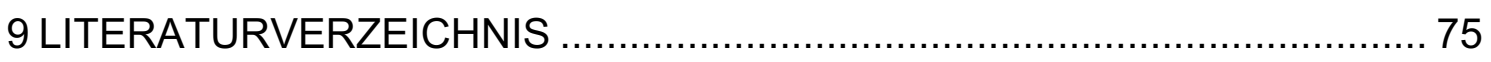




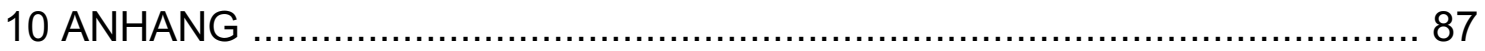

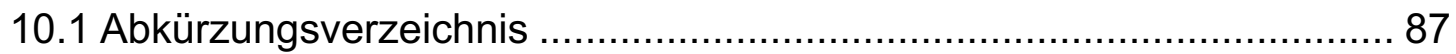

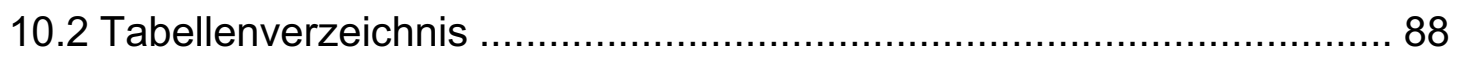

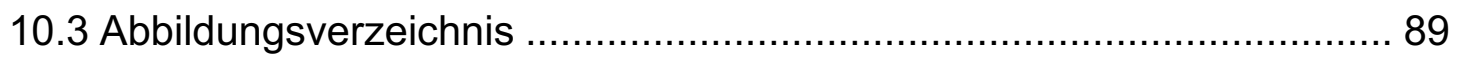

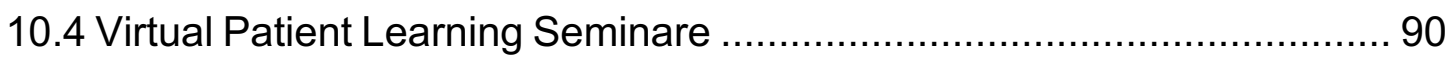

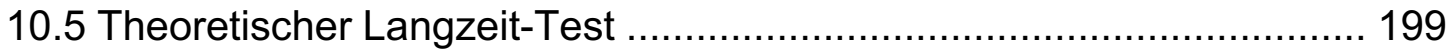

10.6 Theoretischer Kurzzeit-Test ..................................................... 203

10.7 Beispiel Bewertungsbogen im Wahlfach OSCE ............................... 208

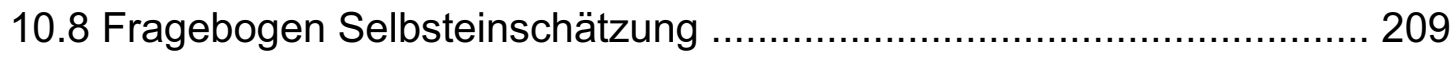

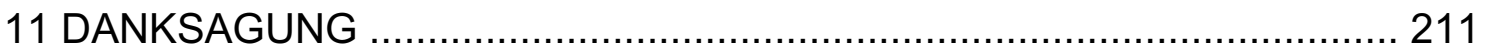




\section{SCHRIFTLICHE ERKLÄRUNG}

Ich erkläre ehrenwörtlich, dass ich die dem Fachbereich Medizin der Johann Wolfgang Goethe-Universität Frankfurt am Main zur Promotionsprüfung eingereichte Dissertation mit dem Titel

Evaluation verschiedener Lehrformate in der Vermittlung allgemeinmedizinischer Basiskompetenzen innerhalb einer studentisch betriebenen Poliklinik

in der Klinik für Mund-, Kiefer- und Plastische Gesichtschirurgie, Universitätsklinikum Frankfurt am Main unter Betreuung und Anleitung von Prof. Dr. Dr. Dr. Robert Sader mit Unterstützung durch Dr. Lukas B. Seifert ohne sonstige Hilfe selbst durchgeführt und bei der Abfassung der Arbeit keine anderen als die in der Dissertation angeführten Hilfsmittel benutzt habe. Darüber hinaus versichere ich, nicht die Hilfe einer kommerziellen Promotionsvermittlung in Anspruch genommen zu haben.

Ich habe bisher an keiner in- oder ausländischen Universität ein Gesuch um Zulassung zur Promotion eingereicht. Die vorliegende Arbeit wurde bisher nicht als Dissertation eingereicht.

Vorliegende Ergebnisse der Arbeit wurden in folgendem Publikationsorgan veröffentlicht:

Lukas B. Seifert, Arda Manap, Jasmina Sterz, Ferdinand M. Gerlach, Robert Sader, A comparison between virtual patient and peer-assisted learning in teaching basic medical knowledge and skills, Electronic Journal of e-Learning, Band 18, Seiten 40-56, 2020 


\section{GENDER ERKLÄRUNG}

Aus Gründen der leichteren Lesbarkeit und zur Erhaltung des Leseflusses wird in der vorliegenden Arbeit die männliche Sprachform verwendet. An dieser Stelle soll darauf hingewiesen sein, dass die ausschließliche Verwendung des Maskulinums als geschlechtsneutral zu betrachten ist und keinesfalls eine Benachteiligung des weiblichen Geschlechtes impliziert. 


\section{ZUSAMMENFASSUNG UND ABSTRACT}

\subsection{Zusammenfassung}

Hintergrund/Zielsetzung: Die Studentische Poliklinik Frankfurt (SP) ist die erste sogenannte Student-run Free Clinic in Deutschland. In ihr versorgen Studenten der Humanmedizin unter ärztlicher Aufsicht nicht-krankenversicherte Patienten. Vor der Tätigkeit in der SP müssen die Studenten ein intensives Vorbereitungsprogramm absolvieren. Dieses Programm ist seit Sommer 2013 als Wahlpflichtfach an der Medizinischen Fakultät der Goethe-Universität Frankfurt curricular verankert. Im Wintersemester 2016/2017 wurde zusätzlich zum bestehenden Peer-assisted Learning Kurs ein web-basierter Virtual Patient Learning Kurs eingeführt.

Ziel dieser Studie war es, die Wirksamkeit von Peer-assisted Learning mit Virtual Patient Learning im Erwerb allgemeinmedizinischer Grundkenntnisse und fertigkeiten zu vergleichen. Betrachtet wurden hierbei unterschiedliche Ebenen des Kompetenzerwerbs: theoretisches Wissen, praktisches Wissen und Selbstevaluation standen im Fokus der Studie.

Methoden: 51 Studenten des fünften Fachsemesters wurden randomisiert in eine Peer-assisted Learning Gruppe (PAL Gruppe; $n=20$ ), eine Virtual Patient Learning Gruppe (VPL Gruppe; $n=20)$ und eine Kontrollgruppe (KG, $n=11)$. Alle Gruppen absolvierten den curricularen Unterricht des ersten klinischen Semesters. Zusätzlich durchlief die PAL Gruppe das Wahlfach der SP im Peerassisted Learning Format. Die VPL Gruppe durchlief das Wahlfach der SP im web-basierten Format mit sogenannten virtuellen Patienten auf der e-Learning Plattform Lernbar der Goethe Universität Frankfurt.

Die Messung des Wissenserwerbs beinhaltete einen theoretischen Vortest und Nachtest (Langzeit-Test) mit je 24 Single-Choice Fragen und theoretische Kurzzeit-Tests nach jedem der Kasuistikseminare mit je fünf Single-Choice Fragen. Der praktische Kompetenzerwerb wurde durch eine curriculare und eine zum Wahlfach gehörende Objective Structured Clinical Examination (OSCE) nach Abschluss der Intervention gemessen. Außerdem schätzten die Studienteilnehmer ihren Wissens- und Kompetenzerwerb vor und nach Teilnahme am Wahlfach der SP mit Hilfe eines Fragebogens ein. Hierfür 
beantworteten sie 34 Fragen anhand einer sechsstufigen Likert-Skala ( 1 = sehr sicher; 6 = überhaupt nicht sicher).

Nach jedem Kasuistikseminar evaluierten die Studenten die jeweilige Kasuistik mit je fünf Fragen anhand einer sechsstufigen Likert-Skala (1 = ich stimme voll zu; 6 = ich stimme überhaupt nicht zu).

Das Signifikanzniveau wurde auf 0.05 festgelegt.

Ergebnisse: Im gesamten theoretischen Nachtest erwarben alle Gruppen (PAL, VPL und KG) einen signifikanten Wissenszuwachs (PAL $p<0.0001$; VPL $p<$ 0.0001; KG $p=0.0156$ ) verglichen mit dem theoretischen Vortest. In allen theoretischen Kurzzeit-Tests wies die VPL Gruppe ein signifikant besseres Ergebnis auf als die PAL Gruppe (Mittelwert PAL = $85.75 \%$; Mittelwert VPL = $90.57 \% ; p=0.0047)$.

Im Wahlfach OSCE zeigte sich kein signifikanter Unterschied zwischen der PAL und VPL Gruppe ( $p=0.5395)$. Im curricularen OSCE zeigte sich kein signifikanter Unterschied zwischen beiden Testgruppen und der KG ( $p=0.4263)$.

In der Selbsteinschätzung nach Intervention schätzte sich die PAL Gruppe in 31 von 34 Items signifikant besser ein als zuvor. Die VPL Gruppe schätzte sich in 25 Items und die KG in 16 der 34 Items signifikant besser ein als zuvor.

Die Kasuistikseminare wurden von der PAL und VPL Gruppe ähnlich bewertet. Die Mediane für die einzelnen Kasuistiken lagen bei 1 oder 2.

Zusammenfassung: Allgemeinmedizinische Grundkenntnisse und Fertigkeiten können mit VPL genauso effektiv vermittelt werden wie mit PAL. Aufgrund der Kosteneffizienz, einer hohen Reproduzierbarkeit und des frei wählbaren Umfangs bezüglich Bearbeitungsort-und Zeit, sollte VPL häufiger in der allgemeinmedizinischen Lehre im Rahmen von Student-run Free Clinics durchgeführt werden. Letztendlich kann dies zu einer verbesserten Behandlungsqualität und Patientenzufriedenheit führen.

Die VPL Seminare sollten dennoch weiterentwickelt werden und besonders im Hinblick auf Feedback an die Studenten moduliert und individualisierter gestaltet werden. 


\subsection{Abstract}

Background: The Studentische Poliklinik (SP) is the first so-called Student-run Free Clinic in Germany where supervised medical students provide free consultation hours to underserved and uninsured patients.

Before working in the consultation hours of SP, students must complete an intensive preparation program. In 2013 , this program has become a curricular elective subject at the Medical Faculty of Goethe-University Frankfurt.

In the winter semester 2016/2017, a web-based virtual patient learning course (VPL) was introduced in addition to the existing peer-assisted learning course (PAL). The aim of this study was to compare the effectiveness of PAL vs. VPL in acquiring basic medical knowledge and skills.

Different levels of competency acquisition were considered: theoretical knowledge, practical knowledge and self-evaluation.

Methods: 51 students of the fifth semester were randomly grouped into a peerassisted learning group (PAL, $n=20$ ), a virtual patient learning group (VPL, $n=$ 20 ) and a control group (KG, $n=11)$. All groups completed the curricular lessons. In addition, the PAL group went through the elective of the SP in the peer-assisted learning format. The VPL group went through the elective of the SP in the webbased format with so-called virtual patients on the e-learning platform Lernbar.

The performance measurement for the theoretical knowledge acquisition included a theoretical pretest and posttest (long-term test) with 24 single-choice questions and a short-term theoretical test after each seminar with five singlechoice questions.

The practical competence was measured by an elective subject belonging and a curricular Objective Structured Clinical Examination (OSCE).

In addition, the study participants evaluated their acquisition of knowledge and competency before and after the elective subject of the SP answering 34 questions each on a six-level Likert scale $(1=$ very confident; $6=$ not confident at all).

After each case study seminar, the students evaluated the case study with five questions each on a six-level Likert scale $(1=$ I totally agree; $6=$ I do not agree at all).

The significance level was set at 0.05 . 
Results: In the theoretical posttest, all groups (PAL, VPL and KG) acquired a significant increase in knowledge (PAL $p<0.0001$; VPL $p<0.0001 ; K G p=$ $0.0156)$ compared to the theoretical pretest. Considering all theoretical short-term tests together, the VPL group showed a significantly better result than the PAL group (mean PAL $=85.75 \%$; mean $\mathrm{VPL}=90.57 \% ; p=0.0047$ ).

An assessment of practical skills in the elective subject associated OSCE showed no differences between the PAL and VPL groups ( $p=0.5395)$. The curricular OSCE showed no significant difference between the two test groups and the control group $(p=0.4263)$.

In the self-assessment after the intervention, the PAL group assessed itself significantly better than before in 31 of 34 items. The VPL group assessed itself significantly better in 25 items and the KG in 16 of 34 items.

Overall, students assessed their learning experience and the comprehensibility of seminars as either "very good" or "good".

Conclusion: Basic medical knowledge and skills can be taught effectively using VPL or PAL. Given the cost-effectiveness, high reproducibility and freedom of time and place, VPL should be performed more often when teaching family medicine in student-run free clinics. Ultimately, this may result in enhanced treatment quality and patient satisfaction.

In future, further research is required about VPL seminars incorporating individualized student feedback. 


\section{EINLEITUNG}

Die vorliegende Arbeit vergleicht erstmals die beiden Lehrformate Peer-assisted Learning (PAL) und Virtual Patient Learning (VPL) im Rahmen einer Student-run Free Clinic in Deutschland. Der Einsatz von neuen Lehrmethoden in der medizinischen Ausbildung hat eine nicht zu unterschätzende Bedeutung für die Patienten-zentrierte Gesundheitsversorgung. In der modernen medizinischen Lehre soll der Lernende im Mittelpunkt stehen und selbstbestimmt lernen können. In dieser Arbeit soll der Nutzen von sogenannten virtuellen Patienten in einem web-basierten Lehrprogramm für die Vermittlung allgemeinmedizinischer Basiskompetenzen gezeigt werden. Die Arbeit behandelt dabei die Frage, ob virtuelle Patienten medizindidaktisch genauso effektiv sind wie das bereits etablierte Peer-assisted Learning Format.

Zunächst wird das Konzept der Studentischen Poliklinik Frankfurt (SP) skizziert und drauffolgend werden die Student-run Free Clinics in den USA betrachtet, um so grundlegende Informationen zum Verständnis der Arbeit zu geben. Anschließend werden die beiden Lehrformate Peer-assisted Learning und Virtual Patient Learning definiert und die Motive zur Entstehung dargestellt. Weiterhin werden ihre wesentlichen Vorteile und Nachteile beleuchtet. Darauf aufbauend wird das Ziel und der Anspruch der Arbeit formuliert. Im Methodenteil werden der genaue Ablauf des Wahlfachs und die Leistungsmessung der teilnehmenden Studenten erklärt. Dem schließen sich die Ergebnisse der durchgeführten Tests an und im letzten Abschnitt der Arbeit werden diese diskutiert.

\subsection{Gründung und Anspruch der Studentischen Poliklinik Frankfurt}

Die Studentische Poliklinik Frankfurt wurde im Sommer 2014 am Fachbereich Medizin der Goethe-Universität nach dreijähriger Vorarbeit durch den Studiendekan und eine engagierte Gruppe von Medizinstudenten in Kooperation mit dem Institut für Allgemeinmedizin und dem Gesundheitsamt der Stadt Frankfurt gegründet. ${ }^{1-3}$

Sie ist die erste studentisch organisierte und geführte Sprechstunde für nichtkrankenversicherte Menschen in Deutschland und wurde ähnlich dem Konzept der US-amerikanischen Student-run Free Clinics aufgebaut. ${ }^{2(\mathrm{p} 515)}$ Anlehnend an 
das Frankfurter Modell wurde im Februar 2018 eine weitere deutsche Studentische Poliklinik in Hamburg eröffnet.4,5 Die Eröffnung einer weiteren Studentischen Poliklinik in Hannover ist für 2020 geplant. $^{6}$

Zweimal wöchentlich bietet die Studentische Poliklinik in Frankfurt eine kostenlose und anonyme allgemeinmedizinische Sprechstunde für Menschen ohne Zugang zum regulären Gesundheitssystem an. Ergänzend zur medizinischen Versorgung wird seit Herbst 2017 in Kooperation mit der University of Applied Science Frankfurt eine Sozialberatung innerhalb der Sprechstunde angeboten.

Die Motive zur Gründung einer solchen medizinischen Einrichtung beruhen zum einen auf Zahlen des statistischen Bundesamtes, welche im Jahre 2011 insgesamt 137.000 Menschen als nicht krankenversichert meldeten. ${ }^{7}$ Kritisch muss hier betrachtet werden, dass nur befragte Personen in die Statistik aufgenommen wurden und man von einer weitaus höheren Dunkelziffer an nichtversicherten Menschen in Deutschland ausgehen kann. Hinzu kommt, dass Frankfurt als Ballungszentrum besonders betroffen ist. Projekte wie die Humanitäre Sprechstunde Frankfurt, die ebenfalls Patienten ohne Krankenversicherung eine medizinische Sprechstunde anbieten, bekräftigen die Nachfrage nach derartigen Projekten. ${ }^{8}$ Schade, Heudorf und Tiarks-Jungk ${ }^{8(p 472)}$ schlussfolgerten in ihrer Datenerfassung zur Humanitären Sprechstunde des Gesundheitsamtes Frankfurt im Jahre 2015: „Unsere Daten zeigen, dass der Bedarf zur Versorgung von Migranten ohne legalen Aufenthaltsstatus oder ohne Krankenversicherungsschutz steigt und diese Menschen auf humanitäre Angebote angewiesen sind."

Zum anderen wurde die studentische Initiative mit der Meinung, dass alle Menschen in Deutschland Zugang zu medizinischer Versorgung erhalten sollten, vom Dekanat tatkräftig unterstützt. Hinzu kommt, dass mit dieser Art von Projekt neue Möglichkeiten der Lehre ausgeschöpft werden sollten. Zusammenfassend füllt die Studentische Poliklinik neben der medizinischen Ausbildung eine klinische Lücke im deutschen Gesundheitssystem. Innerhalb der Sprechstunden werden die Studenten durch erfahrene Fachärzte für Allgemeinmedizin und Innere Medizin begleitet, wodurch eine qualitativ hochwertige Versorgung der Patienten gewährleistet ist. 
Als weitere Maßnahme zur Qualitätssicherung durchlaufen die Studenten vor ihrer Mitarbeit in der Sprechstunde der Studentischen Poliklinik ein ausführliches Vorbereitungsprogramm, welches seit Sommer 2013 als Wahlfach curricular am Fachbereich Medizin der Goethe-Universität Frankfurt akkreditiert ist. ${ }^{2(\mathrm{p} 515), 4}$ Das Wahlfach der Studentischen Poliklinik richtet sich an Studenten des ersten klinischen Semesters und wird seit dem Wintersemester 2013/2014 jährlich durchgeführt. ${ }^{9}$ Bis einschließlich des Wintersemesters 2019/2020 durchliefen bereits 267 Studenten das Wahlfach. Für die herausragende Idee und die Umsetzung, eine hausärztliche Versorgung praxisorientiert und selbstgerichtet zu vermitteln und damit sogar bedürftigen Patienten zielgerichtet zu helfen, erhielt die Studentische Poliklinik Frankfurt im Mai 2017 den hessischen Hochschulpreis für Exzellenz in der Lehre. ${ }^{10}$

Dieses Wahlfach steht im Mittelpunkt der vorliegenden Arbeit und wurde bis zum Wintersemester 2015/2016 im sogenannten Peer-assisted Learning Format durchgeführt.2(p516-517) Im Wintersemester 2016/2017 wurde erstmals parallel hierzu ein e-Learning Format mit sogenannten virtuellen Patienten eingeführt und ist seitdem Bestandteil des Wahlfachs. Dies soll im Methodenteil genauer betrachtet werden.

Die Studentische Poliklinik stellt eine hausärztlich orientierte Einrichtung dar. Folgend beinhaltet das Wahlfach häufige allgemeinmedizinische Beratungsanlässe als Lernziele. Wesentliche Versorgungsschwerpunkte liegen in der Prävention und Therapie von Erkrankungen wie der arteriellen Hypertonie, Herzkreislauferkrankungen, Diabetes Mellitus, Beschwerden des Bewegungsapparates und des Gastrointestinaltraktes, die gleichzeitig auch die häufigsten Konsultationsgründe in der hausärztlichen Versorgung widerspiegeln. Ziel der Studentischen Poliklinik ist zum einen somit eine präventive Arbeit, welche vor dem Hintergrund steigender Behandlungskosten von allgemeinmedizinischen Erkrankungen mehr denn je eine tragende Rolle in der medizinischen Ausbildung spielt. ${ }^{11-13}$ Zum anderen möchte das Projekt Medizinstudenten frühzeitig an die allgemeinmedizinische ambulante Versorgung heranführen und sie für die Lebenswirklichkeit und Probleme nichtkrankenversicherter Menschen in Frankfurt am Main sensibilisieren. 


\subsection{Konzeption der Student-run Free Clinics}

Student-run Free Clinics vereinen die Versorgung minder versorgter Menschen mit einer Lehrpraxis für Medizinstudenten, wie von der Society of Student-run Free Clinics $^{14}$ aus den USA beschrieben wird:

„A student-run free clinic is an institution that provides care at no cost to those in our society that may not otherwise be able to afford such services. These clinics are staffed by volunteers seeking opportunities to provide care to populations such as the uninsured and homeless while simultaneously enrich their education with real-life patient care. "

Mit dieser Definition einer Student-run Free Clinic identifiziert sich auch die Frankfurter Studentische Poliklinik. Die Patienten können dabei wohnungslos oder obdachlos sein, der entscheidende Faktor ist, dass sie nicht krankenversichert sind und innen nur durch eine studentisch geführte Poliklinik ein kostenloser Zugang zu medizinischer Versorgung ermöglicht wird.

Als Vorbild für zahlreiche Student-run Free Clinics in den USA dienten die Student-run Free Clinics der University of California San Diego, welche ab 1997 entstanden. ${ }^{15,16}$ Ellen Beck, Dozentin für Allgemeinmedizin an der University of California San Diego, trug maßgeblich zur Gründung und Etablierung dieser Kliniken bei. ${ }^{16} \mathrm{Ihr}$ zufolge ist Hauptziel der Einrichtungen in San Diego die Förderung und der Erhalt von Gesundheit durch kostenlose medizinische Versorgung, Gesundheitsbildung und Zugang zu sozialen Dienstleistungen. ${ }^{16(\text { (p208) }}$ Mittlerweile sind Student-run Free Clinics in den USA weit verbreitet und existieren an zahlreichen medizinischen Fakultäten. ${ }^{14,17-19} \mathrm{Am}$ jährlich stattfindenden Kongress der Society of Student-run Free Clinics nahmen beispielsweise im Jahre 2019 insgesamt 152 Kliniken teil. ${ }^{14}$

In einer Studie der American Association of Medical Colleges ${ }^{18(\mathrm{p} 2408)}$ wurde für den Zeitraum 2005 bis 2014 eine Verdopplung der Institutionen mit einer Studentrun Free Clinic beobachtet, sodass zu diesem Zeitpunkt an mehr als $75 \%$ aller medizinischen Fakultäten in den USA eine Student-run Free Clinic existierte. Die gleiche Studie fand heraus, dass die häufigsten in Student-run Free Clinics behandelten Krankheitsbilder Diabetes mellitus und Hypertonie seien, ${ }^{18(p 2408-2409)}$ sodass schnell deutlich wird, dass diese Kliniken, ähnlich wie die Studentische Poliklinik in Frankfurt, eine allgemeinmedizinische Grundversorgung anbieten. 
Die Student-run Free Clinics in den USA haben einen Mehrwert in vielerlei Hinsicht. Sie verbessern die Versorgung von marginalisierten Patientengruppen und leisten somit zunehmend einen wichtigen Beitrag zur Gesundheitsversorgung in Nordamerika. ${ }^{13,17,20} \mathrm{Zu}$ erwähnen sei hier die eindrückliche Summe von etwa 27,5 Millionen Menschen ohne Krankenversicherung im Jahre 2018 in den USA, was 8,5\% der USamerikanischen Bevölkerung entspricht und somit eine weit größere Dimension, verglichen zu Deutschland, einnimmt. ${ }^{21}$

Aber auch ein ökonomischer Nutzen lässt sich erkennen. In einer Studie von Arenas et al $^{13}$ konnte gezeigt werden, dass durch präventive Gesundheitsmaßnamen in einer Student-run Free Clinic Kosten eingespart werden könnten. Dies lässt sich möglicherweise auf die insgesamt niedrigen Kosten einer Student-run Free Clinic zurückführen, da die teilnehmenden Studenten ehrenamtlich arbeiten und die Ärzte meist nur eine geringe Bezahlung erhalten.

Student-run Free Clinics bieten einen Raum für das sogenannte Service Learning, welches eine Lernerfahrung beschreibt, die gemeinnützige Arbeit und Lehre mit definierten Lernzielen verknüpft. ${ }^{22}$ Das Service Learning Konzept der Student-run Free Clinics, welches demnach ehrenamtliches Engagement mit Lehre kombiniert, bietet Studenten die frühzeitige Möglichkeit praktische Fähigkeiten des Arztberufes zu erlernen. ${ }^{3(p 464)}$ In Student-run Free Clinics lernen Medizinstudenten drei essentielle Eigenschaften, die motivierend wirken und das Patienten-zentrierte Lernen fördern: Verantwortung, Authentizität und Zusammenarbeit. ${ }^{23(\mathrm{p} 3-5)}$ Die Lernumgebung in Student-run Free Clinics wird als authentisch beschrieben, da die Studenten echte Patienten betreuen und hierbei mit anderen Studenten und Ärzten zusammenarbeiten und gleichzeitig Verantwortung für die Behandlung der Patienten übernehmen. ${ }^{23(\mathrm{p} 4)}$ Verbunden damit ist, dass im Rahmen von Student-run Free Clinics die Autonomie von Medizinstudenten gefördert wird, ${ }^{12}$ was laut Williams und $\mathrm{Deci}^{24(\mathrm{p} 305)} \mathrm{zu}$ einem höheren Selbstwertgefühl der Studenten führt und mit größerem Interesse am Lernen verknüpft ist.

Die frühzeitige praktische Ausbildung und vor allem aber die langfristige Lernerfahrung mit einem langfristigen Patientenmanagement sind Stärken von Student-run Free Clinics. ${ }^{12}$ Dies wird auch seitens der Studenten gelobt. ${ }^{23,25}$ In 
der curricularen medizinischen Ausbildung in Deutschland ist eine solch langfristige Lernerfahrung nicht immer gegeben, zumal sich Famulaturen oder Praktika meist nur über einen Monat oder weniger erstrecken. Ein langfristiges Patientenmanagement kann so nicht erlernt werden. In Student-run Free Clinics können sich die Studenten über einen längeren Zeitraum engagieren und die langfristige Betreuung von Patienten übernehmen. In der Studentischen Poliklinik Frankfurt kann dies beispielsweise während des gesamten klinischen Studienabschnitts der Fall sein. So können die Studenten ihre Patienten teilweise über einen Zeitraum von vier Jahren behandeln und stehen somit ähnlichen Situationen und Herausforderungen gegenüber wie jenen von Hausärzten.

Weiterhin werden die Studenten für die Not marginalisierter Gruppen sensibilisiert und lernen die Bedeutung sozialer Verantwortung kennen. ${ }^{26}$ Durch die Fülle des curricularen Lehrplans an den medizinischen Fakultäten werden häufig die Bedeutung sozialer Einflussfaktoren für Gesundheit nicht ausreichend beleuchtet und marginalisierte Patientengruppen nicht thematisiert. In den Student-run Free Clinics lernen die Studenten also über reines medizinisches Wissen hinaus. Grundkenntnisse über Einflüsse von Gesellschaft und Umwelt auf die Gesundheit zu erlagen, wird auch von der Approbationsordnung für Ärzte vorgeschrieben. ${ }^{27}$ Den Student-run Free Clinics wird in der Vermittlung von Problematiken in der Gesundheitsversorgung eine besondere Rolle zuteil, da sich die Studenten aktiv mit anderen, teils stark benachteiligten Lebenssituationen der Patienten auseinandersetzen. ${ }^{26}$ Ein Großteil, der in Student-run Free Clinics ehrenamtlich arbeitenden Studenten engagiert sich, um Menschen in Not zu helfen. ${ }^{18(p 2408)}$ Dies ist eine sehr wertvolle empathische Eigenschaft für zukünftige Ärzte und kann im Rahmen von Student-run Free Clinics gefördert werden.

Seitens des Patienten ist ebenfalls ein positives Feedback zu beobachten. ${ }^{19,28(p 18)}$ Dass Studenten und Ärzte sich ausreichend Zeit nehmen, wird als sehr angenehm bewertet. ${ }^{28(p 17)}$ Möglicherweise ist dies zum einen auf die fehlende praktische Routine der Studenten und zum anderen auf die Lehre während den Sprechstunden zurückzuführen. Der Patient erfährt somit eine ausgesprochen breite Aufmerksamkeit und steht im Mittelpunkt. Studenten führen meist sehr detaillierte Anamnesen und körperliche Untersuchungen durch, was Patienten sehr wertzuschätzen scheinen. ${ }^{19(p 5)}$ 
Mit dem US-amerikanischen Vorbild der Student-run Free Clinics arbeitet die Studentische Poliklinik an ähnlichen Zielen. Neben allgemeinmedizinischem Wissen soll den Studenten die Wichtigkeit sozialer Einflussfaktoren, Teamarbeit und das Lernen miteinander beigebracht werden. Notwendig hierfür ist eine gezielte und ausführliche Ausbildung und Motivation der Studenten für die Sprechstundenarbeit der Studentischen Poliklinik.

\subsection{Peer-assisted Learning in der Medizin}

\subsubsection{Begriffserklärung}

Peer-assisted Learning (PAL) bedeutet zunächst frei aus dem Englischen übersetzt Peer-unterstütztes Lernen, wobei das Wort Peer unterschiedlich verstanden werden kann. Es kann sowohl Gleichaltriger oder Kollege als auch jemand mit dem gleichen Status/der gleichen Fähigkeit/Ausbildung bedeuten. ${ }^{29}$ Taheri et $\mathrm{al}^{30(\mathrm{p} 1)}$ zufolge unterrichten Personen einer gleichen Gruppe sich gegenseitig. Um den Begriff jedoch besser zu verstehen, eignet sich besonders die Definition von Boud et $\mathrm{al}^{31(p 413)}$, in der PAL als „[...] the use of teaching and learning strategies in which students learn with and from each other without the immediate intervention of a teacher." beschrieben wird. Das von- und miteinander Lernen ist dabei als Kernaspekt zu verstehen. Eine Durchsicht der Literatur ergab verschiedene Formen und Einsatzgebiete des PAL. ${ }^{30(p 1)}$ Meist bieten ältere Studenten ihren jüngeren Kollegen Lehr- und Lernunterstützung an oder übernehmen ganz die Dozententätigkeit. Diese Form entspricht der Rollenverteilung im Wahlfach der Studentischen Poliklinik. In der vorliegenden Arbeit sollen Peer-Tutoren Studenten darstellen, die sich in einem höheren Fachsemester befinden und eine Lehrtätigkeit übernehmen. Diese unterrichten die Studienteilnehmer, welche sich in einem jüngeren Fachsemester befinden.

\subsubsection{Motive zur Entstehung}

Gute klinische Ausbilder und deren Ausbildungsqualität sind fördernde Komponenten für medizinische Lehre. ${ }^{32(p 808)}$ Jedoch stehen den Dozenten und Professoren mit ihrer Lehrtätigkeit mehrere Herausforderungen im Weg. Neben der vorgesehenen Lehrtätigkeit sind sie zeitlich meist stark eingespannt und 
gehen weiteren Aufgaben, wie beispielsweise der klinischen Forschung nach. ${ }^{32,33(\mathrm{p} 59)}$ Zusätzlich zum Zeitmangel lastet auch ein finanzieller Druck auf den medizinischen Fakultäten, was darin resultiert, dass mehrere Studenten

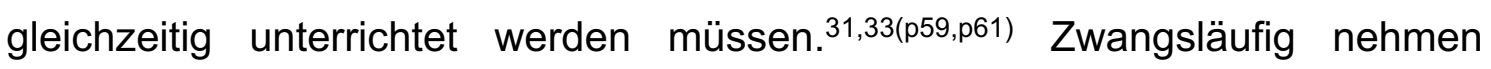
Studenten dann passive Rollen ein und sind nicht aktiv am Unterricht beteiligt. ${ }^{34,35}$ Auch die allgemeine Kompetenz zu Lehren scheint beim medizinischen Personal nicht immer gegeben zu sein. ${ }^{33(p 61)}$ Dem gegenüber ist zu erwähnen, dass eine medizindidaktische Ausbildung in Deutschland an mittlerweile mehreren Fakultäten in unterschiedlichster Form angeboten wird. ${ }^{36}$ Außerdem gewinnen didaktische Qualifizierungsmaßnahmen an Wert und werden bereits auch in einigen Habilitationsordnungen gelistet. ${ }^{36}$ Trotzdem spaltet sich die Meinung über obligatorische medizindidaktische Schulungen, sodass ein allgemein verbindliches Schulungsprogramm in Deutschland derzeit noch fehlt. ${ }^{36}$ Hinzu kommt, dass obligatorische didaktische Maßnahmen als zusätzliche Belastung gesehen werden. ${ }^{37}$ Eine einheitliche Qualifizierung an Lehrenden ist somit nicht immer gegeben.

All diese limitierenden Faktoren für gute Lehre ließen die Universitäten nach neuen Lehrstrategien streben. Das Konzept des Peer-assisted Learning entstand schließlich in den 1960er Jahren. ${ }^{34}$ Treibende Faktoren waren mangelnde Lehrkompetenzen, zeitliche Einschränkungen und finanzieller Druck an den Universitäten. Heute wird PAL in der medizinischen Ausbildung weltweit als akzeptierte Lehrmethode eingesetzt. ${ }^{38,39}$

\subsubsection{Vorteile und Nachteile}

Die zwischenmenschliche Nähe von studentischen Tutoren zu den Lernenden führt dazu, dass diese sich in einer angenehmen Lernatmosphäre befinden und so ein offener Austausch gefördert wird. ${ }^{31,38}$ Wenn ältere Studenten den jüngeren helfen ihre Wissenslücken zu füllen, geschieht dies häufig ohne jegliche Art der Wertung, ${ }^{40}$ was mögliche Ängste mindert und ebenfalls zu einer behüteten Lernumgebung beiträgt. Peer-Tutoren werden oft für zugänglicher gehalten als beispielsweise Ärzte und Professoren, da beide Parteien den gleichen „Status“ besitzen und Hierarchien in den Hintergrund rücken. Im PAL wird insbesondere Wert auf Zusammenarbeit anstelle Wettbewerb gelegt. ${ }^{31(p 415)}$ Das Fehlen einer 
klassischen Autoritätsperson lockert die Lernatmosphäre auf und Studenten können, ohne Angst etwas falsch zu machen, sich im Unterricht einbringen. Zudem lernen die Studenten im PAL das „Lernen wie man lernt“. Sie lernen eigene Lernbedürfnisse zu definieren, Gruppenarbeit selbst zu planen und somit kollektiv Verantwortung zu übernehmen. ${ }^{31(p 416)}$

Die Identifikation mit älteren Studenten scheint ein weiterer wichtiger Motivator zu sein. Sutkin et $\mathrm{al}^{41(\mathrm{p} 457)}$ zufolge geht eine exzellente Lehre über den normalen Unterricht hinaus und ist dadurch gekennzeichnet, dass die Studenten inspiriert, unterstützt und aktiv einbezogen werden. Die Identifikation mit dem Lehrer kann solch bereits erwähnte Inspiration für die Studenten darstellen. ${ }^{41(p 457)}$ So scheint es logisch, dass Studenten sich leichter mit anderen Studenten identifizieren als mit Hochschullehrern. Besonders wenn die Peer-Tutoren denselben Kurs bereits bestanden haben, ist eine indirekte Unterstützung der Studenten zu erkennen. ${ }^{42}$ Die Peer-Tutoren - insbesondere wenn sie den entsprechenden Kurs absolviert haben - sind sensibilisiert für die Bedürfnisse der Lernenden und können somit helfen neue Lernmethoden zu entwickeln, um die jeweiligen Kurse zu optimieren. ${ }^{43(\mathrm{p} 288)}$ Bugaj et $\mathrm{al}^{38}$ beschreiben in diesem Zusammenhang die sogenannte kognitive Kongruenz, welche sich auf die Annahme bezieht, dass studentische Tutoren und die unterrichteten Studenten eine ähnliche Wissensbasis aufweisen, ähnliche Lernerfahrungen teilen und damit auf der "gleichen Wellenlänge“ liegen. ${ }^{38}$ All diese nicht-kognitiven Eigenschaften von studentischen Tutoren scheinen eine besondere Rolle zu spielen und den Kern eines wertvollen klinischen Lehrers auszumachen. ${ }^{41(p p 457-458)}$ Trotzdem ist eine gute Vorbereitung der studentischen Tutoren notwendig, um bessere Lernergebnisse zu erzielen. ${ }^{44(p 64)}$

Der Einsatz von studentischen Tutoren zeigt finanziell einen klaren Vorteil. Zum einen ist die Bezahlung der Studenten kostengünstiger, gleichzeitig sind mehrere Tutoren in der Lage kleinere Studentengruppen zu unterrichten. Zum anderen entlastet dies auch die Dozenten, sodass diese mehr Zeit für die Planung und Vorbereitung ihrer Unterrichtsstunden investieren können. ${ }^{44(p p 64-65)}$

Durch den Einsatz von Peer-Tutoren werden zusätzlich zu den finanziellen Vorteilen die Studenten aktiver in den Unterricht miteinbezogen. ${ }^{34,43(\text { p284) Eine }}$ aktive Rolle im Lernprozess einzunehmen, ist herausfordernd für die Studenten. Sie setzen sich dann bewusster mit den Lerninhalten auseinander. 
Aus wirtschaftlicher und medizindidaktischer Sicht ist PAL eine stark für sich sprechende Lehrmethode. Jedoch ziehen nicht allein die Fakultäten und unterrichteten Studenten ihre Vorteile aus diesem Konzept. Vielmehr lernen die studentischen Tutoren durch das Lehren mit. ${ }^{34,38,44(p 65)}$ Passend dazu wird dies von Ali et al ${ }^{44(\mathrm{p} 65)}$ beschrieben: "Peer tutoring is a two way process and it is equally beneficial for the tutors and learners." Das „Lernen durch Lehren“ ist allerdings kein neues Phänomen. Schon im 17. Jahrhundert schrieb Johann Amos Comenius ${ }^{45(\mathrm{p} 117)}$ in seinem Werk Die große Didaktik: „Wer andere lehrt, der bildet sich selbst.“

Mit der gezielten Vorbereitung jüngere Studenten zu unterrichten, erfolgt die Durchsicht der jeweiligen Thematik erneut und meist mit einem besseren Verständnis. ${ }^{34}$ Die Tutoren festigen somit ihr zuvor gelerntes Wissen und hinterfragen Sachverhalte, die durch einen anderen Blickwinkel erst neu erkannt werden. Um Sachverhalte oder beispielsweise im medizinischen Kontext die körperliche Untersuchung zu unterrichten, müssen die Tutoren sich genauestens mit diesen Themen befasst haben. Verständnisfragen von anderen Studenten können dazu führen, dass bestimmte Inhalte neu aufgerollt und behandelt werden.

Auch werden die zwischenmenschlichen und kommunikativen Fähigkeiten der Tutoren gestärkt, ${ }^{34}$ sodass sie mehr Selbstsicherheit und Verantwortungsbewusstsein erlangen.

An dieser Stelle muss ein möglicher Nachteil im Peer-assisted Learning Format genannt werden. Studentische Tutoren haben individuelle Eigenschaften und vermitteln Sachinhalte womöglich unterschiedlich. So kann es sein, dass in der Vermittlung Inkongruenzen entstehen und die Wissensvermittlung nicht immer auf gleicher Ebene stattfindet. Hier zeigt sich noch einmal, dass das gezielte Schulen von Tutoren sehr wichtig ist, um eventuelle Ungleichheiten auszugleichen. Demgegenüber kann aber genau die individuelle Art zu Lehren auch vorteilhaft sein, da die unterrichteten Studenten ebenfalls unterschiedlich auf Tutoren reagieren und somit auch Studenten aktiviert werden können, die sonst eher passivere Rollen einnehmen.

Im PAL ist sowohl der Lernende als auch der Lehrende auf eine gute Zusammenarbeit angewiesen. So üben sich beide Parteien in Teamarbeit,, ${ }^{31(p 415)}$ welches als eines der Kernmerkmale für eine gute klinische Arbeit gilt. Die 
Partizipation von Medizinstudenten in Student-run Free Clinics lehrt ebenfalls eine teambasierte Patientenversorgung. ${ }^{26}$ So kann daraus abgeleitet werden, dass PAL und die Lehre in Student-run Free Clinics synergistisch wirken können und besonders dazu geeignet sind, Teamarbeit zu fördern.

\subsubsection{Anwendungsbereiche}

In den Student-run Free Clinics ist PAL ein integraler Bestandteil. 2,17,46 Der Großteil des Unterrichts an diesen Kliniken wird von Studenten durchgeführt und stellt somit Formen von PAL dar. ${ }^{17(p 355)}$ Der Studentenunterricht wird seitens der Studenten als eine der größten Stärken von Student-run Free Clinics gelobt. ${ }^{18(p 2408)}$

So lernen die Studenten im PAL insbesondere klinisch praktischen Fertigkeiten. ${ }^{47-49}$ Außerdem eignet sich das Lehrkonzept für das Erlernen der körperlichen Untersuchung und Anamneseerhebung. ${ }^{2(p p 518-521)}$

\subsection{Virtual Patient Learning in der Medizin}

\subsubsection{Begriffserklärung}

Virtual Patient Learning (VPL) kann aus dem Englischen frei als das Lernen mit virtuellen Patienten übersetzt werden. Kononowicz et $\mathrm{al}^{50(\mathrm{p} 4)}$ zufolge wurde der Begriff um virtuelle Patienten erstmals 1991 im Bereich der medizinischen Lehre eingeführt und ist seither zunehmender Gegenstand der wissenschaftlichen Literatur. Es wird beschrieben, dass verschiedene Definitionen und ein heterogenes Verständnis von virtuellen Patienten und deren Zweck insbesondere in der Lehre herrschen. ${ }^{50(p 1)}$

Um jedoch einer Erklärung des Begriffs näherzukommen, eignet sich die Definition der Association of American Medical Colleges ${ }^{51(p 7)} \mathrm{zu}$ virtuellen Patienten:

„A specific type of computer-based program that simulates real-life clinical scenarios; learners emulate the roles of health care providers to obtain a history, conduct a physical exam, and make diagnostic and therapeutic decisions." Virtuelle Patienten scheinen somit anders formuliert ein Computerprogramm darzustellen, in welchem reale klinische Szenarien simuliert werden und die 
Studenten die Rolle von Ärzten übernehmen um eigenständig eine Anamnese zu erheben, eine körperliche Untersuchung durchzuführen und selbstständig diagnostische und therapeutische Entscheidungen zu treffen. ${ }^{51(\mathrm{pp6} 6-8)}$

In der vorliegenden Arbeit ist unter virtuellen Patienten ein imaginärer Patient zu verstehen, der in einem web-basierten Fallseminar befragt, untersucht und behandelt wird. Der virtuelle Patient wird mithilfe von Bildmaterial und Textabschnitten illustriert.

Ellaway ${ }^{52\left(\mathrm{p}^{2}\right)}$ beschreibt mehrere Möglichkeiten der Anwendung von virtuellen Patienten im medizinischen Ausbildungssektor: Der Lernende kann demnach verschiedene Rollen einnehmen, wie zum Beispiel die des Arztes, aber auch die des Patienten oder von Angehörigen. Weiterhin bieten virtuelle Patienten laut Ellaway ${ }^{52(p 2)}$ unterschiedliche Lehrformen an. So kann zum Beispiel ein vorgeschriebenes Szenario die Studenten in Einzelschritten durch einen Fall führen oder die Studenten begutachten und bewerten einen bestehenden Patientenfall. ${ }^{52(\mathrm{p} 2)}$ Zusätzlich kann der Lernende unabhängig oder unter Anleitung eines Tutors arbeiten. ${ }^{52(\mathrm{p} 2)}$ Es sind somit verschiedene Einsatzmöglichkeiten von virtuellen Patienten mit einer breiten Auswahl an Umsetzungen gegeben. Die Heterogenität von eingesetzten virtuellen Patienten spiegelt sich auch in der Literatur wider. ${ }^{50,52(\mathrm{p} 1)}$

Interessant ist natürlich zu überlegen, welche Merkmale vorhanden sein müssen, um den Studenten erfolgreich Lerninhalte beizubringen. In einem Review von Issenberg et al $^{53(\mathrm{pp} 21-24)}$ wurden zehn Aspekte herausgearbeitet, die zum effektiven Lernen im Bezug zu medizinischen Simulationen beitragen. Diese Merkmale können jedoch zum Teil auch auf das Lernen mit virtuellen Patienten übertragen werden. ${ }^{51(\mathrm{p9})}$ Das Feedback ist, neben häufigen Wiederholungen und multiplen Lernstrategien, eines der Hauptkriterien für effektives Lernen. ${ }^{53(p 10)}$ Es sollte insbesondere in der klinischen Ausbildung von Medizinstudenten nicht vernachlässigt werden. ${ }^{54-59}$ Individuelles und konstruktives Feedback darf auch in web-basierten Lehrformaten mit virtuellen Patienten nicht fehlen. 


\subsubsection{Motive zur Entstehung}

Im deutschen Gesundheitswesen ist ein zunehmender ökonomischer Druck mit gesteigerten Fallzahlen und kürzeren Liegezeiten zu beobachten. ${ }^{60,61}$

Der praktische Unterricht am Patienten - unabhängig ob von Peer-Tutoren oder Ärzten durchgeführt - wird daher häufig als Belastung empfunden. Gleichzeitig spielen die Ansprüche an die Patientensicherheit mit der Sorge um Behandlungsfehler eine wichtige Rolle, so König et al. ${ }^{62(p 556)}$. Diese ökonomischen und ethischen Gründe tragen dazu bei, dass immer weniger Zeit und Motivation für Lehre am Patientenbett vorhanden ist. Eine zurückgehende Beteiligung der Medizinstudenten an der klinischen Praxis, welches sich aus Gründen der Patientensicherheit und steigender Zahl an Studenten ergibt, kann sich schnell nachteilig auf den Lernprozess auswirken. ${ }^{63}$ Hinzu kommt, dass vor allem junge Ärzte in Weiterbildung kaum medizindidaktische Kompetenz besitzen und insbesondere zu Beginn ihrer ärztlichen Tätigkeit mit sich selbst und dem Erwerb eigener Kenntnisse und Fertigkeiten beschäftigt sind. ${ }^{62(p 555-556)}$

Auch im Marburger Bund Monitor $2017^{64}$ wurde erfasst, dass eine flächendeckende Arbeitsüberlastung, Personalmangel und zunehmende bürokratische Hürden im klinischen Alltag im Mittelpunkt stünden. In einer Arbeit im Rahmen eines Didaktik-Trainings der Chirurgischen Arbeitsgemeinschaft Lehre untersuchte die Autorengruppe um König et al. ${ }^{62}$ die Hindernisse für klinische Lehrtätigkeit. Auch sie führten den Personalmangel als eines der Haupterschwernisse für klinische Lehre an. ${ }^{62(p 554, \mathrm{p} 556)}$ So ist schnell zu erkennen, dass im klinischen Alltag weniger Zeit für Lehre bleibt.

König et al. ${ }^{62(10555-556)}$ beschreiben in ihrem Artikel auch, dass oft keine geeigneten Lehrinfrastrukturen existieren (Bibliotheken, PC-Arbeitsplätze, Seminarräume etc.).

Seitens der Studenten scheint zusätzlich ein höherer Anspruch an die Lehre vorhanden zu sein. Die sogenannte Generation Y (Jahrgänge 1981 bis 1996) verlangt eine optimale Ausbildung und gute Supervision. ${ }^{65(\mathrm{p} 2)} \mathrm{Hinzu}$ kommt, dass diese Generation mit der Nutzung elektronischer Medien aufgewachsen ist und die Nutzung von Laptops und Smartphones zum Alltag und den natürlichen Lernressourcen gezählt werden. ${ }^{65(\mathrm{p} 5)}$ 
Zum einen aus der Not heraus die ökonomischen und ethischen Hindernisse zu umgehen und zum anderen, um der neuen Generation an Studenten gerecht zu werden, sind neue elektronische Lehrmethoden notwendig. Es erscheint nicht wunderlich, dass virtuelle Patienten als alternative Lehrmethode in der medizinischen Ausbildung immer beliebter werden. ${ }^{66(p 1001)}$ So beschreiben auch Consorti et al ${ }^{66(p 1001)}$ in ihrer Metaanalyse: „VPs [Virtual Patients, Anm. d. Verf.] can provide a way to overcome the reduced student access to real patients, as well as a structured and safe environment for students to practice."

\subsubsection{Vorteile und Nachteile}

Wie jedes Lehrformat in der medizinischen Lehre, haben auch virtuelle Patienten zahlreiche Vor-und Nachteile, die in der Tabelle 1 zusammengefasst werden. Ein starkes Argument zur Nutzung von virtuellen Patienten ist der leichte Zugang und die Unabhängigkeit. ${ }^{51(p 7)}$ Gerade durch die neue Generation von Studenten wird der leichte Zugang deutlich hervorgehoben: das Lernen orientiert sich stark an elektronischen Medien. ${ }^{65(\mathrm{p} 2)}$ Der Association of American Medical Colleges ${ }^{51(\mathrm{p} 6)}$ zufolge umgehen moderne Technologien wie das Lernen mit virtuellen Patienten den „Platzmangel und die Tutor-Verfügbarkeit“. So können die Studenten an jedem Ort lernen, ohne auf freie Räumlichkeiten angewiesen zu sein. ${ }^{67}$ Zudem besteht eine zeitliche Unabhängigkeit. Die Studenten können in ihrer individuellen Lerngeschwindigkeit die Lerneinheiten bearbeiten und sich für bestimmte Themen, falls nötig, mehr Zeit nehmen. ${ }^{67}$ So können die individuellen Lernvoraussetzungen des Anwenders berücksichtigt werden. ${ }^{68}$ Die Menge an Lehrinhalten kann leicht erhöht werden und Interessen vertieft werden.

Darüber hinaus verlagern virtuelle Patienten den Fokus der Inhalte von einer „Lehrer-zentrieten“ auf eine „Lerner-zentriete“ Perspektive. ${ }^{69(p 156)}$ Dies bedeutet, dass die Studenten aktive Entscheidungen und Handlungen durchführen, anstatt passiv einem Fallbeispiel beizuwohnen. ${ }^{70,71(p 1592)}$ Der Lernende, als „aktiver Teilnehmer“ anstelle eines „passiven Zuschauers“, ist eines der zehn genannten Aspekte von Issenberg et $\mathrm{al}^{53(\mathrm{pp} 22-23)}$ für effektives Lernen. Ferner kann der Lernende neben der Rolle des Arztes verschiedene weitere Rollen einnehmen, wie beispielsweise die des Patienten oder von Angehörigen und so seinen Horizont erweitern. ${ }^{52(\mathrm{p} 2)}$ 
Die neue Art des Lernens mit virtuellen Patienten bedeutet auch, dass Studenten ermutigt werden, Verantwortung für medizinische Entscheidungen zu übernehmen. Dabei können die Studenten Eigenverantwortung und Selbstständigkeit erlernen. Daraus kann, wie bereits beschrieben, die Motivation zu Lernen steigen. ${ }^{23(p p 7-8)}$ Trotzdem müssen die Studenten auch eine gewisse Selbstdisziplin zeigen.

Consorti et al ${ }^{66(p 1005)}$ erläutern zudem in ihrer Metaanalyse: „In being challenging for the learner, VPs [Virtual Patients, Anm. d. Verf.] are believed to foster an active learning and hence they should be consistently compared to other active methods of teaching/learning, particularly if the intervention is addressed to the acquisition of clinical competence and not to factual knowledge." Virtuelle Patienten fördern somit durch ihre herausfordernde Art ein aktives Auseinandersetzen mit den Lehrinhalten. Gormley et al $^{72}$ schlussfolgerten, dass die Herausforderung als Kliniker zu agieren und Verantwortung für echte Fälle zu übernehmen eines der besten Wege sei zu lernen.

Virtuelle Patienten bieten den Studenten ein sicheres Lernumfeld, ${ }^{66(p 1001)}$ dies ist eine weitere wichtige Eigenschaft für effektives Lernen. ${ }^{53(p 10)}$ Sowohl alltägliche Situationen als auch seltene kritische Ereignisse können in einer sicheren und kontrollierten Umgebung einstudiert werden ohne negative Konsequenzen zu fürchten. ${ }^{51(\mathrm{pp} 5-6)}$

Den Studenten bleibt überlassen, ob sie in Gruppenarbeit oder einzeln lernen, wodurch ebenfalls den individuellen Bedürfnissen nachgegangen wird. Weiterhin bieten virtuelle Patienten eine gute Visualisierung. Beispielsweise können mehr Inhalte im direkten Blickfeld erfasst werden oder der Lernprozess angezeigt werden, um den Studenten eine bessere Struktur mitgeben zu können.

Weiterhin sind laufende Kosten geringer und eine größere Anzahl an Studenten kann gleichzeitig unterrichtet werden. Beispielsweise fallen die Kosten für mehrere Tutoren weg. Anzumerken ist hier jedoch, dass anfangs zum Aufbau und Etablierung von Lerneinheiten mit virtuellen Patienten ein höherer zeitlichinhaltlicher Aufwand notwendig sind. 51(p7)

Ebenfalls ist hervorzuheben, dass mit virtuellen Patienten eine standardisierte Lehrqualität und somit eine einheitlich vergleichbare Lehrerfahrung aller Teilnehmer gewährleistet werden kann. ${ }^{67}$ Die Lehrqualität schwankt somit nicht 
von Tutor zu Tutor, was als möglicher Nachteil im Peer-assisted Learning Format genannt wurde.

Ein weiterer Punkt, der für den Einsatz von virtuellen Patienten spricht, ist die weitaus größere Menge an Fällen, die ein Student durcharbeiten kann als es womöglich in seiner normalen klinischen Ausbildung der Fall sein würde. ${ }^{73}$ Virtuelle Patienten bieten somit in der medizinischen Ausbildung eine sehr einzigartige Möglichkeit in qualitativer und quantitativer Weise die Studenten strukturiert in der klinischen Entscheidungsfindung zu schulen. ${ }^{66,73}$

\begin{tabular}{|l|ll|}
\hline \multicolumn{1}{|c|}{ Vorteile } & \multicolumn{1}{c|}{ Nachteile } \\
\hline - Räumliche Unabhängigkeit & $-\quad \begin{array}{l}\text { Praktische Fertigkeiten können } \\
\text { nicht direkt beurteilt werden }\end{array}$ \\
- Zeitliche Unabhängigkeit & - Selbstdisziplin notwendig \\
- Eigenverantwortlichkeit und & - Bildschirmarbeit ersetzt keinen \\
- Selbstständigkeit & echten Patienten \\
- Bichere Lernumgebung & - Anfänglich hoher inhaltlicher \\
- Herausforderung & Aufwand \\
- Standardisierte Qualität & \\
- Geringe laufende Kosten & \\
- Größere Anzahl an & \\
& Patientenszenarien als in der & \\
klinischen Ausbildung möglich & \\
\hline
\end{tabular}

Tabelle 1: Vorteile und Nachteile von virtuellen Patienten

\subsubsection{Anwendungsbereiche}

Trotz einer beträchtlichen Anzahl an ersten Veröffentlichungen über virtuelle Patienten, wurden nur wenige Studien in randomisiertem und kontrolliertem Rahmen durchgeführt. ${ }^{67,74,75}$ In einer ersten Metaanalyse von Cook et al ${ }^{71(p 1599)}$ konnte gezeigt werden, dass das Lehrangebot mit virtuellen Patienten zusätzlich zum bestehenden curricularen Unterricht einen Zuwachs an Wissen, klinischem Denken und Fertigkeiten mit sich brachte. Dies ist unter anderem mit den zuvor genannten zahlreichen Vorteilen zu erklären. Cook et $\mathrm{al}^{71(\mathrm{p} 1600)}$ schlussfolgerten: „Evidence does not indicate superiority of virtual patients over other training methods, but allowing for the uncertainty of the $\mathrm{Cl}$ [confidence interval, Anm. d. Verf.] and imperfections of the outcome measures, they may be noninferior in 
some instances." Demnach sind virtuelle Patienten den traditionelleren Lehrmethoden nicht unterlegen. ${ }^{63(\mathrm{p} 1600)}$. Dies wirft jedoch die Frage in den Raum, ob sie nicht auch eine alternative Form darstellen könnten.

Die Metaanalyse von Consorti et al ${ }^{66(p 1002)}$ ging dieser Fragestellung tiefgründiger nach und konnte 12 randomisiert kontrollierte Studien finden, die virtuelle Patienten zu medizinischen Lehrzwecken nutzten. Die Analyse der Studien zeigte dabei einen klaren positiven Effekt für virtuelle Patienten, verglichen mit anderen Lehrmethoden. ${ }^{66(p 1005)}$ Sie stellten zudem fest, dass virtuelle Patienten als Ergänzung zum bestehenden Lehrangebot, aber auch als Alternative sehr geeignet waren. ${ }^{66(p 1005)}$

In der zuvor genannten Definition der Association of American Medical Colleges $^{51(\mathrm{p} 7)}$ steht vor allem das klinische Denken (Clinical Reasoning), welches eine der Kernkompetenzen von Ärzten dargestellt, im Vordergrund und nimmt einen zentralen Platz in der medizinischen Ausbildung ein. ${ }^{76-78}$ Es ist daher verständlich, dass sich ein erheblicher Teil, der in der medizinischen Ausbildung eingesetzten virtuellen Patienten, auf den Erwerb von Fähigkeiten zum Clinical Reasoning konzentriert. ${ }^{50,71,79-81}$

Der Begriff Clinical Reasoning lässt sich schwer in das Deutsche übersetzen und wurde in dieser Arbeit als klinisches Denken vereinfacht. Hierunter lassen sich „Denkprozesse von klinisch tätigen Personen verstehen, die darauf abzielen, eine klinische Entscheidung zu treffen".82 Diese Denkprozesse und Entscheidungen zielen darauf ab Diagnosen zu stellen. ${ }^{83}$ Virtuelle Patienten eignen sich, um den Weg von Symptomen zur Diagnose-Stellung und damit das klinische Denken zu lehren. Dies lässt sich darauf zurückführen, dass virtuelle Patienten die Möglichkeit bieten eine sehr große Bandbreite an Patientenszenarien durchzugehen. ${ }^{73}$ So können sehr viele diagnostische Wege und Entscheidungsfindungen durchdacht werden.

Neben dem klinischen Denken finden sich jedoch auch einige Studien zum Nutzen von virtuellen Patienten in den Bereichen Kommunikationsfähigkeit und Anamneseerhebung. ${ }^{74,84,85}$

Um die Nutzung von elektronischen Lehrangeboten an medizinischen Fakultäten zu betrachten, eignet sich die Plattform Learning Resource Server Medizin $(\text { LRSMed })^{86}$, welche als Suchmaschine für multimediale Lehr-und Lernmodule in der Medizin genutzt werden kann. Mit dieser Suchmaschine sind alle im Web frei 
verfügbaren Lehr-und Lernmodule abrufbar, die sowohl deutschsprachige als auch englischsprachige Module einschließen. ${ }^{87}$ Der Homepage zufolge wurden zur letzten Aktualisierung im Juli 2014 insgesamt 1818 kostenfreie Lehr-und Lernangebote registriert. ${ }^{86}$ Die Angebote benötigen keine lokalen Installationen oder proprietären Komponenten, sodass sie online problemlos zugänglich sind. ${ }^{86}$ Bei der Suche nach Häufigkeiten der Ressourcen in den jeweiligen klinischen Fachgebieten zeigte sich, dass die Innere Medizin (Endokrinologie, Gastroenterologie, Hämatologie und Onkologie, Infektiologie, Kardiologie, Nephrologie, Pneumologie und Rheumatologie) mit insgesamt 506 Suchergebnissen am weitaus häufigsten vertreten ist. Auch die Radiologie (186 Ergebnisse), Allgemeinmedizin (123 Ergebnisse) und Pädiatrie (103 Ergebnisse) waren häufig vertretene klinische Fächer. Jedoch handelte es sich um jegliche Formen elektronischer Module, sodass auch Audiodateien, Lehrbücher oder Bildmaterial hinzuzählten. Etwa die Hälfte der Lehrangebote waren elektronische Lehrbücher oder Skripte. ${ }^{87} \mathrm{Um}$ eine Idee über die Menge an deutschsprachigen Modulen zu erhalten, die virtuellen Patienten am nächsten kommen, wurden in der Suchmaschine als Anwendungstypen „Fallbeispiele“, „Simulationen“ und "Virtuelle Darstellung“ eingegeben. Zusätzlich wurde die Suche auf deutschsprachige Ergebnisse im Fachgebiet der Allgemeinmedizin eingeschränkt. Diese Suche ergab sechs Ergebnisse, die anschließend genauer inspiziert wurden. Besonders interessant und erwähnenswert ist der INMEDA Simulator $^{88}$ der CompuGroup Medical Deutschland AG, TELEMED ${ }^{89}$, welche eine webbasierte e-Learning Plattform darstellt. Dieser Simulator stellt eine graphisch gestaltete Klinik mit verschiedenen Fachdisziplinen dar, in welcher der Benutzer virtuelle Patienten behandelt. ${ }^{89}$ Besonders ansprechend ist die visuelle Gestaltung dieses Online-Portals. Um Patienten zu behandeln, kann der Nutzer im Eingangsbereich der virtuellen Klinik auf der Beschilderung neben den Aufzügen eine der 17 Fachrichtungen wählen, gelangt dann in den Wartebereich und anschließend in den Untersuchungsraum, um dort einen virtuellen Patientenfall zu bearbeiten. ${ }^{88} \mathrm{Im}$ Untersuchungsraum werden dem Benutzer schließen die Ziele des Falls genannt und der Benutzer kann Anamnese und körperliche Untersuchungen durchführen. Falls nötig kann eine weitere Diagnostik veranlasst werden. ${ }^{88}$ Es stehen dabei Expertenmeinungen, Hinweise und visuelle Hilfen zur Verfügung, die den Benutzer durch den Fall führen. ${ }^{88}$ 
Der Simulator fand dahingehend so positive Resonanz, dass er beispielsweise auch von der Baden-Württembergischen Landesärztekammer als zertifizierte Fortbildung zur Ärztlichen Leichenschau angeboten wurde. ${ }^{90}$ Der Simulator kann von jedem Computer genutzt werden und benötigt keine lokale Installation, sodass er auch gut von zuhause aus genutzt werden kann und jederzeit zur Verfügung steht. Er ist eine der wenigen Anwendungen mit virtuellen Patienten in Deutschland, die benutzerfreundlich und vor allem interaktiv eine medizinische Lehrmethode darstellen.

Insgesamt scheint das Angebot an virtuellen Patienten im deutschsprachen Raum jedoch noch nicht gut erforscht zu sein. In der Literaturrecherche konnte bisher keine Anwendung von virtuellen Patienten zur Lehrezwecken im Setting von Student-run Free Clinics gefunden werden.

\subsection{Zielsetzung der Studie}

Ziel der vorliegenden Arbeit ist der Vergleich der beiden Lehrformate Peerassisted Learning und Virtual Patient Learning im Rahmen einer Student-run Free Clinic. Eine gründliche Sichtung der bisherigen Literatur zum Thema zeigte keine Studien, die diesem Vergleich nachgingen. Somit zeigt diese Arbeit eine erste Gegenüberstellung der beiden Lehrformate in einer Student-run Free Clinic. Betrachtet werden verschiedene Dimensionen des Lernerfolgs der Studenten. Untersucht wird, ob es einen Unterschied im theoretischen oder praktischen Wissens- und Kompetenzerwerb gibt. Die Studenten schätzen zudem ihre erworbenen Kompetenzen subjektiv ein. Außerdem werden die beiden Lehrmethoden von den Studenten bewertet. Die genauen Methoden werden im folgenden Abschnitt genauer betrachtet.

Die Hypothese der vorliegenden Arbeit ist, dass web-basiertes Lernen mit virtuellen Patienten im Wahlfach der Studentischen Poliklinik genauso effektiv ist, wie das bereits etablierte Peer-assisted Learning Format. 


\section{METHODIK}

\subsection{Gruppenzusammensetzung}

51 Studenten des ersten klinischen Semesters im Fach Humanmedizin (fünftes Fachsemester) wurden in eine Peer-assisted Learning Studiengruppe (PAL Gruppe; $n=20$ ) und eine Web-basierte Virtual Patient Learning Studiengruppe (VPL Gruppe; $n=20$ ) randomisiert (Abb. 1). Eine Kontrollgruppe (KG; $n=11$ ) absolvierte nur den curricularen Unterricht des ersten klinischen Semesters (Abb. 1). Alle Studienteilnehmer erklärten sich mit ihrer Anmeldung für das Wahlfach der Studentischen Poliklinik Frankfurt bereit, an der Studie und der anonymen Auswertung ihrer Prüfungsergebnisse und Evaluationen teilzunehmen.

Die zwei Studiengruppen durchliefen im Wintersemester 2016/2017 im Zeitraum Oktober 2016 bis April 2017 das Wahlfach der Studentischen Poliklinik zusätzlich zum curricularen Unterricht des Fachbereichs Medizin der Goethe-Universität.

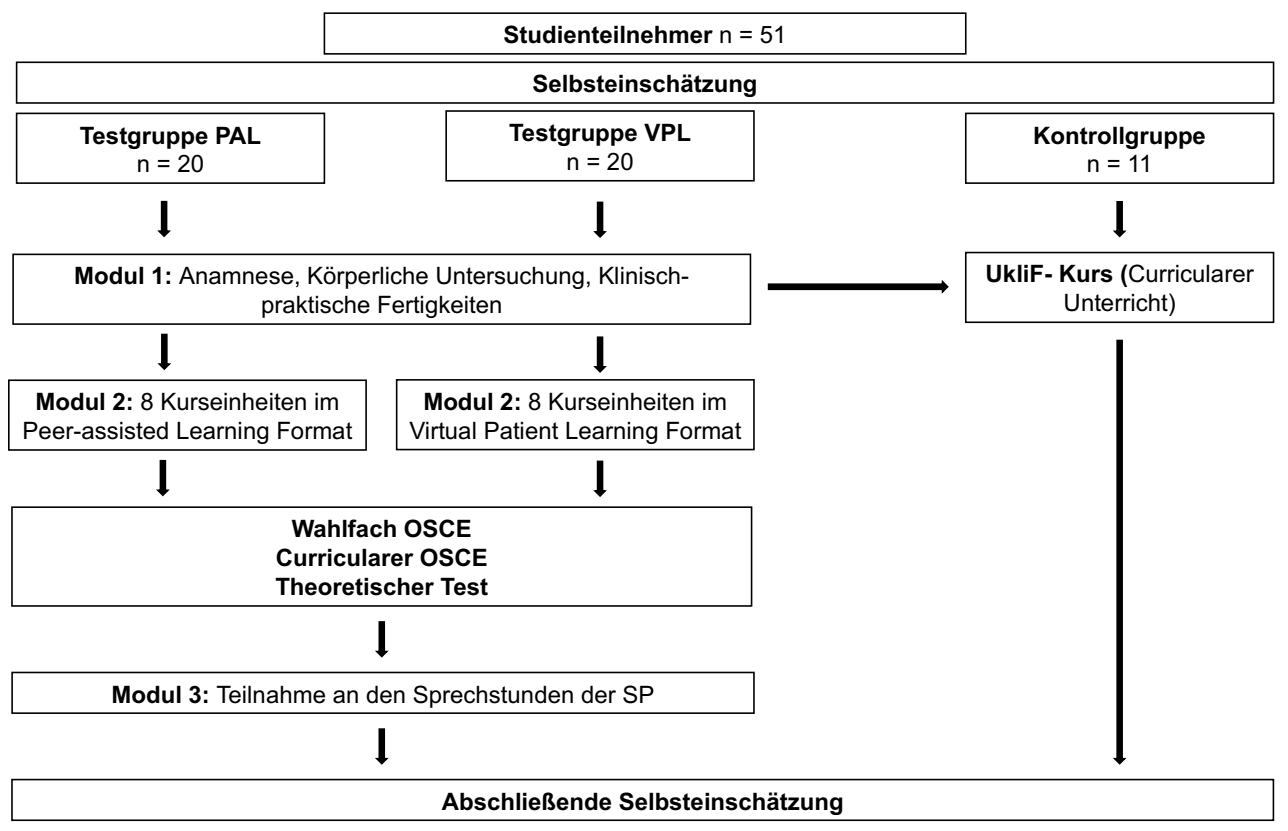

Abbildung 1: Studiendesign 


\subsection{Aufbau des Wahlfachs der Studentischen Poliklinik Frankfurt}

Das Wahlfach der Studentischen Poliklinik setzte sich aus drei unterschiedlichen Modulen zusammen (Abb. 1, Tab. 2).

Zunächst wurden beide Testgruppen $(n=40)$ im ersten Modul an einem zweitägigen Kurs in klinisch-praktischen Fertigkeiten, in den Grundlagen der körperlichen Untersuchung und der Anamneseerhebung geschult (Abb. 1, Tab. 2). Die Vermittlung der Lehrinhalte erfolgte durch erfahrene und geschulte Tutoren höherer Semester und in Kleingruppen (ein Tutor für drei bis vier Studenten). Jeder Student hatte die Gelegenheit unter Anleitung selbstständig die unterschiedlichen Untersuchungsmethoden zu erlernen und einzuüben.

Der Kurs umfasste sechs eineinhalbstündige Unterrichtseinheiten zu folgenden Bereichen: Untersuchung des Bewegungsapparates, Kardiale und pulmonale Untersuchung, Abdominelle Untersuchung, SchwangerschaftsvorsorgeUntersuchung, klinisch-praktische Fertigkeiten, strukturierte Anamneseerhebung und Kontakt und psychosozialer Umgang mit Personen ohne Krankenversicherung (Tab. 2).

Das zweite Modul (Kasuistikseminar) bestand aus acht, wöchentlich stattfindenden Lerneinheiten zu ausgewählten allgemeinmedizinischen Beratungsanlässen (Tab. 2). Diese wurden anhand einer fiktiven PatientenKasuistik mit dazugehöriger Anamneseerhebung, Untersuchungstechniken, differentialdiagnostischen Abwägungen und möglichen Therapieformen unterrichtet. Unter dem Begriff Kasuistik soll in dieser Arbeit eine medizinische Fallbetrachtung verstanden werden.

Das Seminar wurde für die PAL Gruppe im Peer-assisted Learning Stil gehalten. Die Tutoren wurden vor Beginn der Seminare inhaltlich und didaktisch geschult. Die VPL Gruppe hingegen absolvierte die gleichen Kasuistiken über die eLearning Plattform Lernbar der Goethe Universität Frankfurt. ${ }^{91,92}$

Die Kasuistikseminare bezogen sich auf folgende Leitsymptome: Anhaltender Husten, Bauchschmerz, Dyspnoe und Leistungsminderung, Kopfschmerz, Kreuzschmerz, Polyurie (Diabetes), Schwangerschaft und arterielle Hypertonie (Tab. 2).

Nach einer schriftlich-theoretischen Prüfung (Kapitel 6.3.1) und einer praktischen Prüfung (Kapitel 6.3.2) in Form einer Objective Structured Clinical Examination 
(OSCE), ${ }^{93-95}$ konnten die Studenten im Rahmen des dritten Wahlfachmoduls aktiv an der Sprechstunde der Studentischen Poliklinik teilnehmen (Abb. 1, Tab. 2). Dabei absolvierten sie mindestens einen Termin an der Patientenanmeldung/ Rezeption, um möglichst viele organisatorische und administrative Aufgabenbereiche der Sprechstunde kennenzulernen und mindestens drei Termine als sogenannte Juniors an der Seite von erfahreneren Studenten (Seniors).

\begin{tabular}{|c|c|c|}
\hline Modul & $\begin{array}{c}\text { Art der } \\
\text { Durchführung }\end{array}$ & Lernziele \\
\hline \multirow{6}{*}{1} & \multirow{6}{*}{$\begin{array}{l}\text { Peer-assisted } \\
\text { Learning Format } \\
\text { (Kleingruppen von 3-4 } \\
\text { Studenten pro Tutor) }\end{array}$} & $\begin{array}{l}\text { Untersuchung Wirbelsäule, Hüftgelenk, } \\
\text { Kniegelenk, Sprunggelenk, Schultergelenk, } \\
\text { Dermatome und Kennmuskeln }\end{array}$ \\
\hline & & Kardiopulmonale Untersuchung \\
\hline & & Abdominelle Untersuchung \\
\hline & & $\begin{array}{l}\text { Routineuntersuchung in der Schwangerschaft, } \\
\text { Mutterpass ausstellen/ führen, }\end{array}$ \\
\hline & & $\begin{array}{l}\text { Legen eines Venenverweilkatheters, } \\
\text { Blutentnahme, Einzelknopfnaht }\end{array}$ \\
\hline & & $\begin{array}{l}\text { Anamnese/ Gesprächsführung/ Psychosozialer } \\
\text { Umgang mit Menschen ohne } \\
\text { Krankenversicherung }\end{array}$ \\
\hline \multirow{8}{*}{2} & \multirow{8}{*}{$\begin{array}{l}\text { Peer-assisted } \\
\text { Learning Format oder } \\
\text { Virtual Patients } \\
\text { Learning Format }\end{array}$} & $\begin{array}{l}\text { Anhaltender Husten - Tuberkulose und } \\
\text { Differentialdiagnosen }\end{array}$ \\
\hline & & $\begin{array}{l}\text { Bauchschmerz - Chronische Gastritis und } \\
\text { Differentialdiagnosen }\end{array}$ \\
\hline & & $\begin{array}{l}\text { Belastungsdyspnoe und Leistungsminderung - } \\
\text { Anämieformen und Differentialdiagnosen }\end{array}$ \\
\hline & & $\begin{array}{l}\text { Kopfschmerz - Primäre Kopfschmerzen und Red } \\
\text { Flags }\end{array}$ \\
\hline & & $\begin{array}{l}\text { Kreuzschmerz- Unkomplizierte Kreuzschmerzen } \\
\text { und Red Flags }\end{array}$ \\
\hline & & $\begin{array}{l}\text { Polyurie - Diabetes mellitus Typ I und II, } \\
\text { Metabolisches Syndrom }\end{array}$ \\
\hline & & $\begin{array}{l}\text { Schwangerschaft - Vorsorge-I } \\
\text { Routineuntersuchungen, Mutterpass, } \\
\text { Komplikationen in der Schwangerschaft }\end{array}$ \\
\hline & & Arterielle Hypertonie \\
\hline
\end{tabular}




\begin{tabular}{|l|l|l|}
\hline \multirow{5}{*}{3} & $\begin{array}{l}\text { Symptomorientiere Anamnese und körperliche } \\
\text { Untersuchung }\end{array}$ \\
\cline { 3 - 3 } & $\begin{array}{l}\text { Selbstständige } \\
\text { Durchführung als } \\
\text { Junior angeleitet } \\
\text { durch einen Senior } \\
\text { oder Arzt }\end{array}$ & $\begin{array}{l}\text { Erhebung Vitalparameter (Blutdruck, Puls, } \\
\text { Temperatur), Messung des Blutzuckers, } \\
\text { Blutentnahme }\end{array}$ \\
\cline { 3 - 3 } & EKG - Erstellen und Befunden \\
\cline { 3 - 4 } & Patientenvorstellung \\
\cline { 3 - 4 } & Befundbesprechung, Therapieplanung \\
\cline { 3 - 4 } & $\begin{array}{l}\text { Administrative Tätigkeiten - Patientenaufnahme, } \\
\text { Erstellen von Rezepten und Überweisungen, } \\
\text { Terminvereinbarung }\end{array}$ \\
\hline
\end{tabular}

Tabelle 2: Lernziele der einzelnen Wahlfachmodule der Studentischen Poliklinik

\subsubsection{Aufbau des Peer-assisted Learning Seminars}

Die Studenten der PAL Gruppe wurden einmal wöchentlich in einer Gruppe von maximal zehn Studenten im Rahmen einer gemeinsamen Fallbesprechung durch Tutoren betreut. Die Tutoren befanden sich in einem höheren Fachsemester und wurden zuvor zur Thematik geschult. Das Tutorenteam setzte sich aus vier Studenten zusammen, von denen jeder zwei Seminare hielt. Ein Seminar erstreckte sich in etwa über zwei Stunden. Unterstützend zur Vermittlung der Lehrinhalte wurden Präsentationen mithilfe von Microsoft Power Point ${ }^{96}$ erstellt. Zu Beginn des Seminars wurde ein fiktiver Patientenfall mit einem bestimmten Leitsymptom vorgestellt (Abb. 2). Zu jedem Fall erhob ein Student eine strukturierte Anamnese, wobei der Tutor den Patienten simulierte. Die Anamneseerhebung wurde schließlich in der Gruppe gemeinsam besprochen und gegebenenfalls komplettiert. Der durchführende Student bekam Feedback durch Kommilitonen und durch den Tutor. In dieser Phase des Seminars wurden die Kursteilnehmer trainiert auf bestimmte anamnestisch erhobene Schlüsselworte des Patienten zu achten und die entsprechende körperliche Untersuchung einzuleiten. 


\section{In deine Praxis...}

...kommt eine 67-jährige Patientin. Bisher kennst du sie noch nicht. Zunächst möchtest du eine detaillierte Anamnese durchführen.

Die Patientin heißt Anna Gramm und auf deine offene Frage hin, weshalb sie sich in deiner Praxis vorstellt, fängt sie an zu berichten:

„Ich habe starke Bauchschmerzen seit 3 Monaten. Ich habe bereits Ibuprofen genommen, das hat hin und wieder etwas geholfen. Allerdings fühle ich mich immer schwächer. Oft bin ich sehr müde und schaffe es kaum die Treppen hoch. Und nun ist der Schmerz viel stärker."

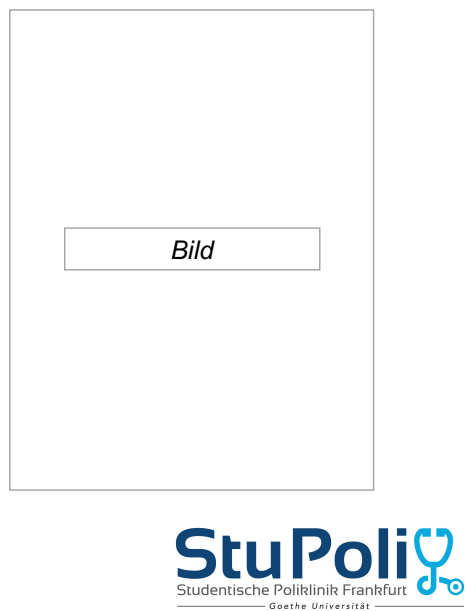

Abbildung 2: Einführende Folie zum PAL Seminar Bauchschmerz

Ein weiterer Student führte daraufhin eine symptomorientierte körperliche Untersuchung an einem anderen Kursteilnehmer durch.

Aufbauend auf Modul 1 konnten die Studenten somit ihre zuvor erworbenen praktischen Fertigkeiten vertiefen. Neben den praktischen Kompetenzen im Bereich der Anamneseerhebung und der symptomorientierten körperlichen Untersuchung, erarbeiteten sich die Studenten gemeinsam und anhand der weiteren Patientenkasuistik wichtige Differentialdiagnosen und ein sinnvoll diagnostisches Vorgehen. Verschiedene diagnostische Methoden wurden hinsichtlich ihrer klinischen Relevanz gemeinsam in der Gruppe besprochen. In der Kasuistik mit dem Thema Bauchschmerz beispielsweise wurden unter anderem die abdominelle Sonographie (Abb. 3) und Gastroduodenoskopie besprochen. Um den Studenten außerdem eine Vorstellung über die Symptomentstehung und Klinik der behandelten Erkrankungen zu geben, wurden grundlegende pathophysiologische Mechanismen (hier im Beispiel „Bauchschmerz“ und Gastritis die Grundlagen der Entzündung) vermittelt. Mit der Reihenfolge „Leitsymptom/ Klinik - Anamnese und körperliche Untersuchung Differentialdiagnosen und Verdachtsdiagnose - weiterführende Diagnostik und Überlegung der Pathophysiologie“" wurden die Studenten an das klinische Denken und Handeln herangeführt. 


\section{Sonogramm der Patientin}

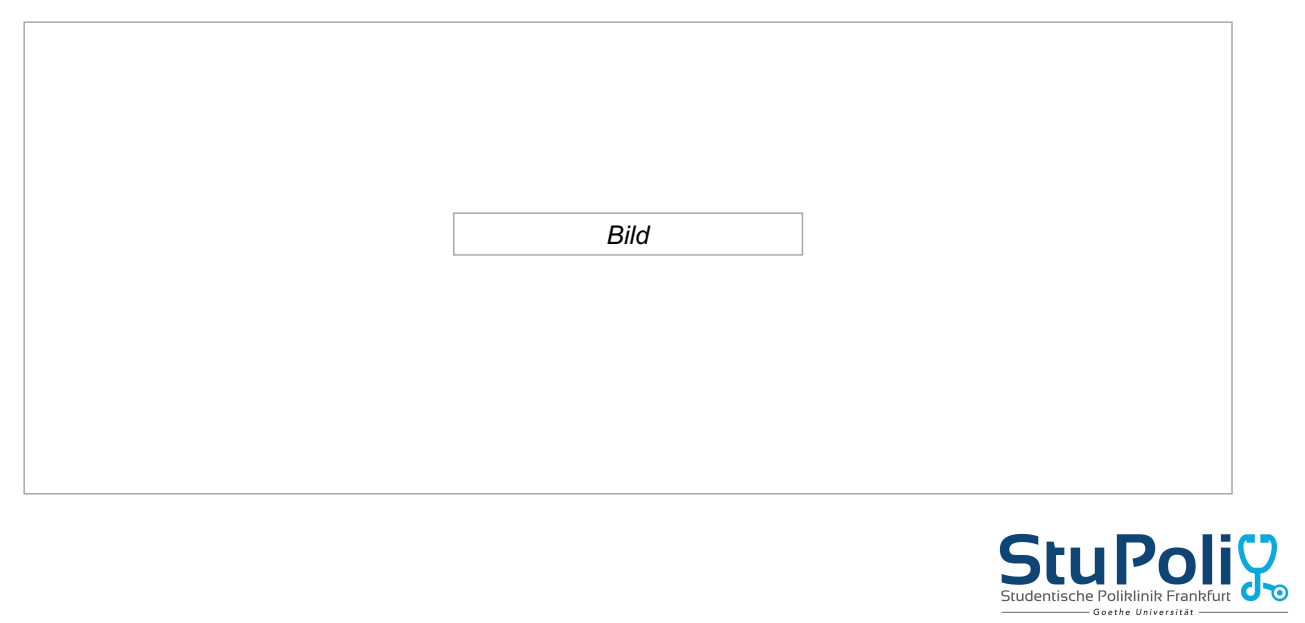

Abbildung 3: Weiterführende Diagnostik im PAL Seminar Bauchschmerz

Die Teilnehmer der PAL Gruppe erarbeiteten sich weiterhin die geeigneten Therapien zu den behandelten Krankheiten (Abb. 4).

Am Ende eines jeden Seminars beantworteten die Studenten fünf thematisch passende Single-Choice Fragen, um den kurzzeitigen Lernerfolg zu messen und den Studenten ein Feedback über die erlernten Inhalte zu geben. Die Fragen waren größtenteils dem Fragenkatalog des Instituts für medizinische und pharmazeutische Prüfungsfragen (IMPP) entnommen. ${ }^{97}$

\section{H. Pylori Eradikationstherapie}

\begin{tabular}{|lllll|}
\hline Therapieform & PPI & AB1 & AB2 & Bemerkung \\
\hline French Triple & Pantoprazol & Clarithromycin & Amoxicillin & Hohe Eradikationsrate \\
\hline Italian Triple & Pantoprazol & Clarithromycin & Metronidazol & $\begin{array}{l}\text { Geringere } \\
\text { Eradikationsrate, } \\
\text { anwendbar bei } \\
\text { Penicillinallergie }\end{array}$ \\
\hline $\begin{array}{l}\text { Bismuth- } \\
\text { Quadrupeltherapie }\end{array}$ & Pantoprazol & Tetracyclin & Metronidazol & + Bismuth \\
& & & \\
& & & \\
\hline
\end{tabular}

Diese Drei Therapieformen sind die derzeitigen Erstlinientherapien!

\section{StuPolig}

Abbildung 4: Folie zur Therapie im PAL Seminar Bauchschmerz 


\subsubsection{Aufbau des Virtual Patient Learning Seminars}

Die Teilnehmer der VPL Gruppe absolvierten das zweite Wahlfachmodul online auf der e-Learning Plattform Lernbar der Goethe-Universität Frankfurt (Abb. 5). ${ }^{91,92}$ Dabei wurde den Studenten wöchentlich ein virtueller Patientenfall zur Bearbeitung freigeschaltet. Die Lehrinhalte waren identisch zu den PAL Patientenfällen (Abb. 5).

Der Zeitraum und Umfang der Bearbeitung blieb den Studenten individuell überlassen und wurde lediglich auf den Bearbeitungszeitraum innerhalb der jeweiligen Woche begrenzt. Damit waren verschieden lange Bearbeitungszeiten und Bearbeitungsgeschwindigkeiten möglich. Zusätzlich konnten bestimmte Themenbereiche durch die Studenten persönlich vertieft betrachtet werden.

Außerdem wurde den Studenten freigestellt sich in Gruppen zusammenzusetzten oder allein die virtuellen Patienten-Kasuistiken zu bearbeiten. Der Ort der Bearbeitung war ebenso frei wählbar. Der Aufbau jedes Falles bezog sich auf das reguläre Kasuistikseminar im PAL Stil und begann somit ebenfalls mit einem Fallbeispiel (Abb. 6). Es entstanden nur geringfügige Unterschiede im Aufbau der Kasuistik, um die fehlende Interaktion mit einem studentischen Tutor auszugleichen.

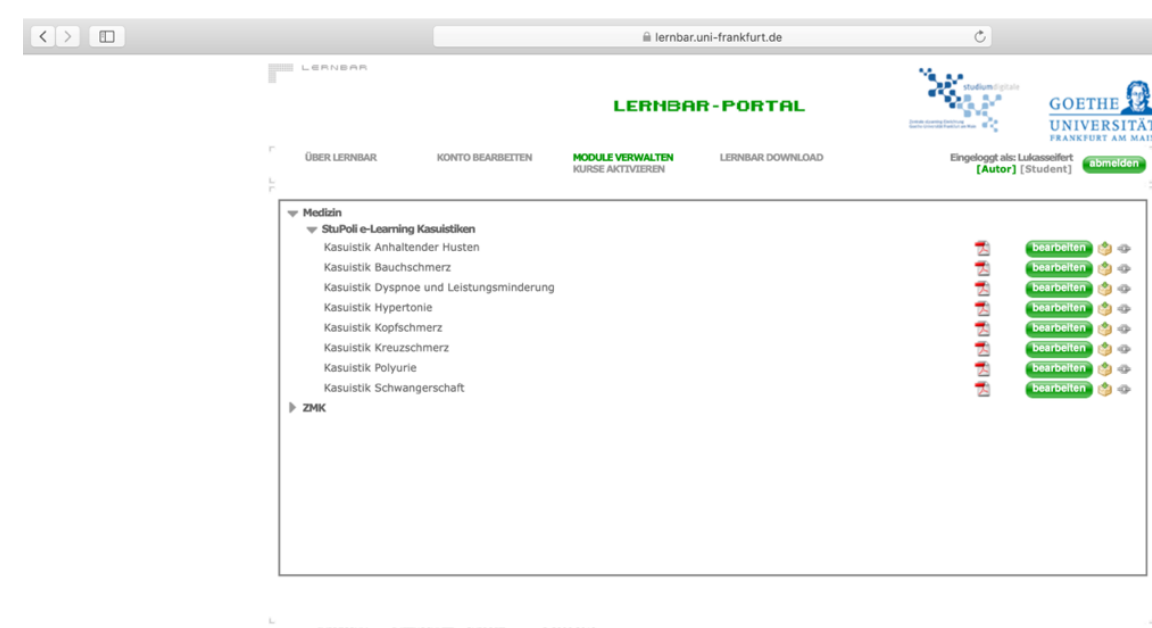

Abbildung 5: e-Learning Plattform Lernbar - Kasuistiken 

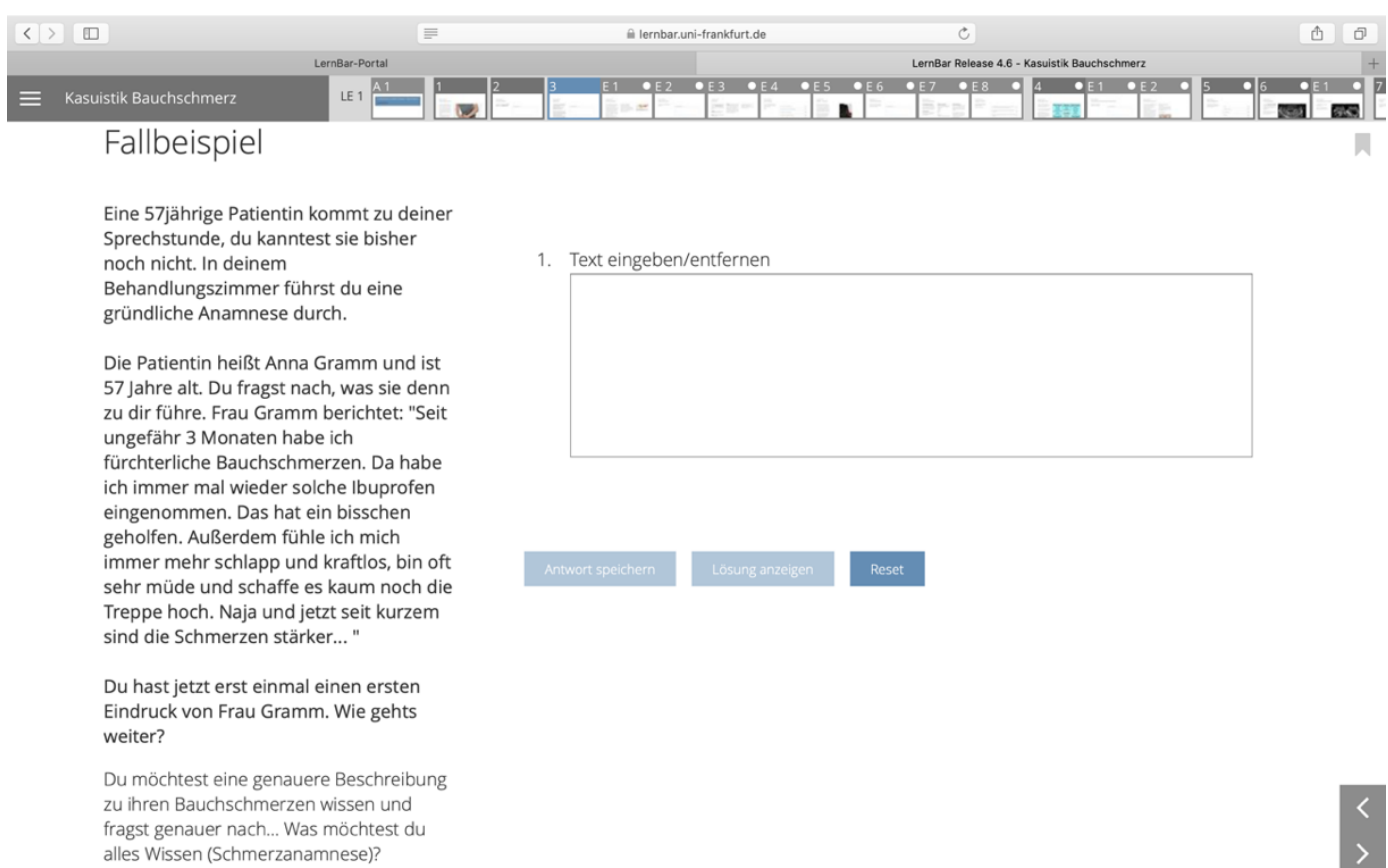

Eine 57jahrige Patientin kommt zu dein

Behandlungszimmer führst du ein

gründliche Anamnese durch.

immer mehr schlapp und kraftlos, bin oft

fragst genauer nach. Was möchtest du

alles Wissen (Schmerzanamnese)

Abbildung 6: Einführung in das VPL Seminar Bauchschmerz

Um die Prozedur von Anamnese und körperlicher Untersuchung möglichst informativ darzustellen, wurden entsprechend Zwischenfragen gestellt. Unter anderem wurden Freitextaufgaben oder Single -und Multiple-Choice Fragen genutzt (Abb. 7, Abb. 8). Anschließend wurden die Lösungsansätze diskutiert und verschiedene Antworten auf Richtigkeit geprüft. Wurde die Frage richtig beantwortet, so wurden die Studenten gelobt und ermutigt. Außerdem wurde erklärt weshalb die Antwort richtig war. Falls Fragen nicht korrekt beantwortet wurden, folgte eine Erklärung zur gewählten Antwort (Abb. 8). Somit konnten die Studenten nachvollziehen, worin ihr eventueller Fehler bestand oder ob sie zum Beispiel an alle relevanten Differentialdiagnosen gedacht hatten. Diese Form von Feedback sollte die Funktion des Tutors aus dem PAL Seminar simulieren. 


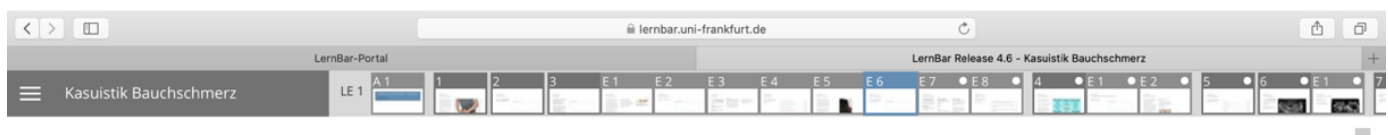

Kasuistik Bauchschmerz

noch mehr Anamnese

Was möchtest du in deiner Anamnese noch

alles wissen? Zähle alle Punkte auf, die du

Frau Gramm fragst.
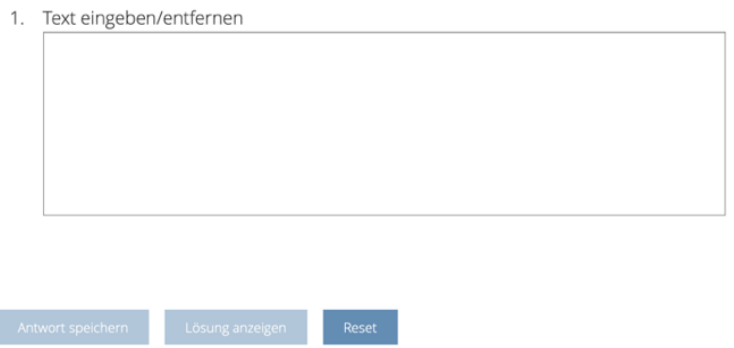

Abbildung 7: Freitextaufgabe aus dem VPL Seminar Bauchschmerz

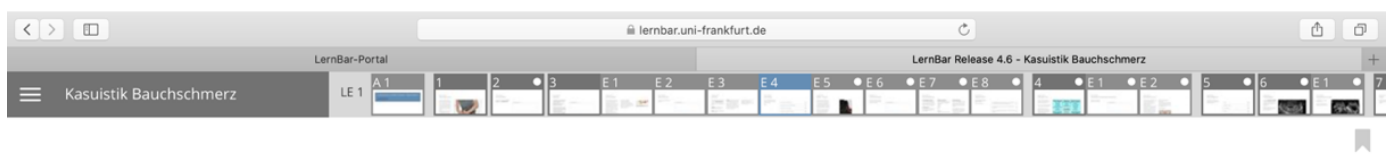

Kasuistik Bauchschmerz

Zwischenfrage

Wie nennt man die Beschreibung des Stuhls von Frau Gramm? Und für was könnte dies sprechen?

VTipp

Frau Gramm hat berichtet, dass ihr Stuhl schwarz sei.

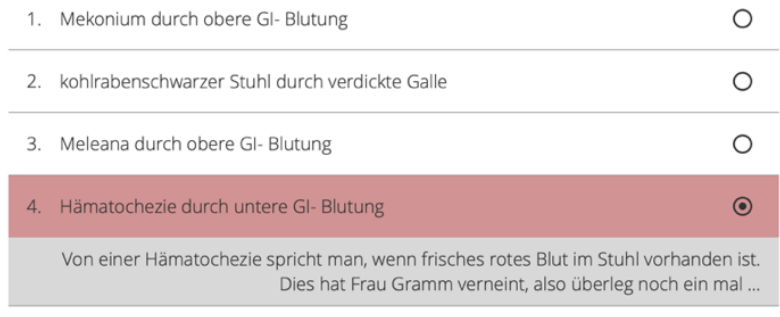

5. Meleana durch eingedickte Galle und bakterielle Entzündung

O

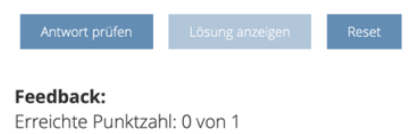

Abbildung 8: Angegebene Falschantwort mit dazugehöriger Erklärung im VPL Seminar Bauchschmerz

Durch den Wechsel von Fragen und Informationen zum Fallbeispiel und zu den jeweiligen allgemeinmedizinischen Themen, konnten sich die Studenten durch das Seminar arbeiten und die relevanten Lerninhalte aneignen. 
Um den praktischen Anteil des regulären Kasuistikseminars zu ersetzen wurden Blickdiagnosen und Lehrvideos zu den körperlichen Untersuchungen verwendet (Abb. 9). Außerdem wurde auf eventuelle weiterführende Literatur hingewiesen. Am Ende jeder Kasuistik wurden den Studenten ebenfalls die fünf Single-Choice Fragen gestellt wie in der PAL Gruppe, um den kurzzeitigen Lernerfolg zu messen und den Studenten ein Feedback zu geben.

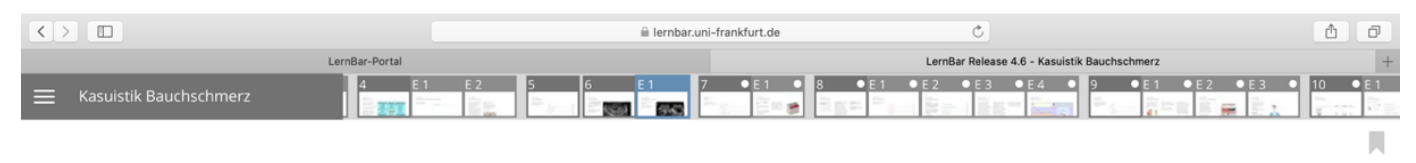

Kasuistik Bauchschmerz

Sonographie von Frau Gramm

Frau Gramm hat keinen normalen Sonograpie-Befund.

Man erkennt eine angeschwollene Magenschleimhaut und Ulzerationen

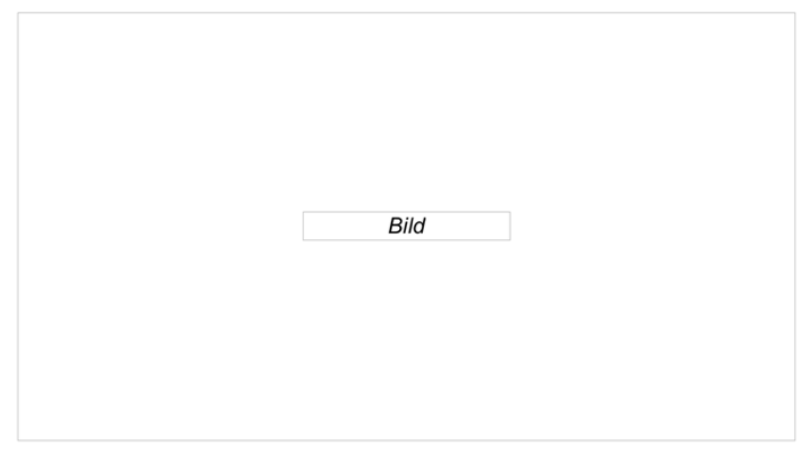

Abbildung 9: Ultraschallbild Abdomen im VPL Seminar Bauchschmerz

\subsubsection{Aufbau der Sprechstunde}

Die Sprechstunde der Studentischen Poliklinik (Modul 3) fand in den Räumlichkeiten des Gesundheitsamtes Frankfurt statt.

Eine Sprechstunde war auf vier Stunden ausgelegt und teilte sich in drei Abschnitte auf. Im ersten Teil - vor der eigentlichen Sprechstunde mit Patienten

- fand eine Vorbesprechung der einbestellten Patienten statt. Hier wurden Laborbefunde, Vorstellungsgründe und mögliches weiteres Vorgehen besprochen. Zusätzlich erfolgte eine 30 bis 40-minütige Lehreinheit (Teaching) zu ausgewählten allgemeinmedizinischen Themen. Das Teaching wurde von einem Studenten eines höheren Semesters gehalten und richtete sich insbesondere an die Wahlfachteilnehmer, die als sogenannte Juniors fungierten. 
Nach der Vorbesprechung fand der zweite Teil der Sprechstunde statt. Dieser stellte die eigentliche Sprechstunde, in der Patienten aufgenommen wurden, dar. An der Anmeldung arbeitete ein Student mit dem sogenannten Clinic Manager (studentischer Leiter der Sprechstunde) zusammen und nahm die Patienten auf. Erfragt wurden unter anderem die Patientendaten und der Vorstellungsgrund. Die Aufgaben an der Anmeldung umfassten organisatorische und administrative Funktionen (Tab. 2). Zwei Teams aus je einem Junior und einem so genannten Senior (Studenten ab dem 8. Fachsemester) untersuchten anschließend die Patienten in einem der zwei Untersuchungszimmer. Gemeinsam wurde eine ausführliche Anamnese erhoben, die Patienten wurden zielorientiert körperlich untersucht und gegebenenfalls weiterführende diagnostische Methoden angewandt (Tab. 2). Gemeinsam wurden eine Verdachtsdiagnose und mögliche Differentialdiagnosen ermittelt und Therapieoptionen besprochen. Die Studenten stellten den Patienten anschließend einem Lehrarzt vor und besprachen mit inm den weiteren Behandlungsverlauf des Patienten.

Nach Behandlung aller Patienten erfolgte eine Nachbesprechung im gesamten Team, welches den dritten Abschnitt der Sprechstunde darstellt. Die Juniors führten dabei zu jedem ihrer Patienten eine Patientenvorstellung durch (Tab. 2). Offen gebliebene Fragen konnten hier geklärt werden. Gemeinsam wurde das weitere Prozedere der einzelnen Patienten besprochen.

\subsubsection{Aufbau des curricularen Unterrichts}

Im ersten klinischen Semester Medizin (fünftes Fachsemester) erhielten die Studenten einführende Veranstaltungen, welche folgende Vorlesungen beinhalteten: Einführung Chirurgie, Einführung Innere Medizin, Erste Ärztliche Hilfe (1.Teil), Radiologie und Strahlenschutz/ Strahlentherapie, Hygiene/ Mikrobiologie/ Virologie, Allgemeine Pathologie, Allgemeine Pharmakologie und Toxikologie. ${ }^{98}$ Pflichtseminare wurden in Biomathematik, Hygiene/ Mikrobiologie/ Virologie, Radiologie und Strahlenschutz, Einführung in die Pathologie, Allgemeine Pharmakologie und Toxikologie (Grundlagen der Pharmakologie einschl. Theoretische Pathophysiologie) und in der Inneren Medizin als Untersuchungskurs klinischer Fächer (UkliF) gehalten. ${ }^{98}$ Im UkliF erlernten die Studenten innerhalb von acht Kursterminen unter Anleitung eines Arztes die 
Erhebung einer Anamnese, Patientenvorstellung, allgemeine Untersuchung, Vitalzeichenbestimmung, Untersuchung von Kopf/ Hals, Untersuchung von Thorax/ Lunge, Untersuchung des Lymphsystems, Herz- KreislaufUntersuchung, Untersuchung des Abdomens und Rückens, Untersuchung des Gefäßsystems, Untersuchung des Bewegungsapparates und die Untersuchung des Nervensystems. Während dieser Termine erlernten die Studenten zum einen die vorab aufgezählten Untersuchungsmethoden in Kleingruppen untereinander, zum anderen mussten sie Patienten untersuchen und vorstellen.

Die theoretische Leistungsüberprüfung der Studenten des ersten klinischen Semesters erfolgte schließlich durch eine Semesterabschlussklausur, welche Single-Choice Fragen zu den Fächern Innere Medizin (35 Fragen), Chirurgie (20 Fragen), Hygiene/ Mikrobiologie/ Virologie (30 Fragen), Allgemeine Pathologie (30 Fragen), Biometrie (10 Fragen), Erste Ärztliche Hilfe (10 Fragen) und Radiologie/ Strahlenschutz (30 Fragen) umfasste. ${ }^{99}$

Mithilfe eines OSCEs wurde der praktische Kompetenzerwerb geprüft. Der OSCE wurde als Parcours mit standardisierten Stationen aufgebaut, wobei für jede Station standardisierte Aufgaben absolviert wurden. Die Aufgaben hierbei spiegelten ärztliche Tätigkeiten wider und sollten möglichst objektivierbar sein, um von sogenannten Observern (Dozenten) bewertet zu werden. ${ }^{93}$ Dieser curriculare OSCE beinhaltete insgesamt sechs Stationen: eine Anamneseerhebung, zwei Untersuchungen der Inneren Medizin (Angiologie, Gastroenterologie, Hämatologie, Kardiologie, Pneumologie), eine neurologische Untersuchung, eine Patientenvorstellung und eine sogenannte Flurstation/ Feedbackstation.

\subsection{Leistungsmessung}

Um einen Wissenszuwachs objektiv messen zu können und um die Studenten möglichst optimal auf ihren Einsatz in der Sprechstunde der Studentischen Poliklinik vorzubereiten, wurden verschiedene theoretische und praktische Leistungsüberprüfungen angewandt. Zudem wurden die Studenten gebeten ihren eigenen Wissenszuwachs subjektiv anhand eines standardisierten Fragebogens zu bewerten. 

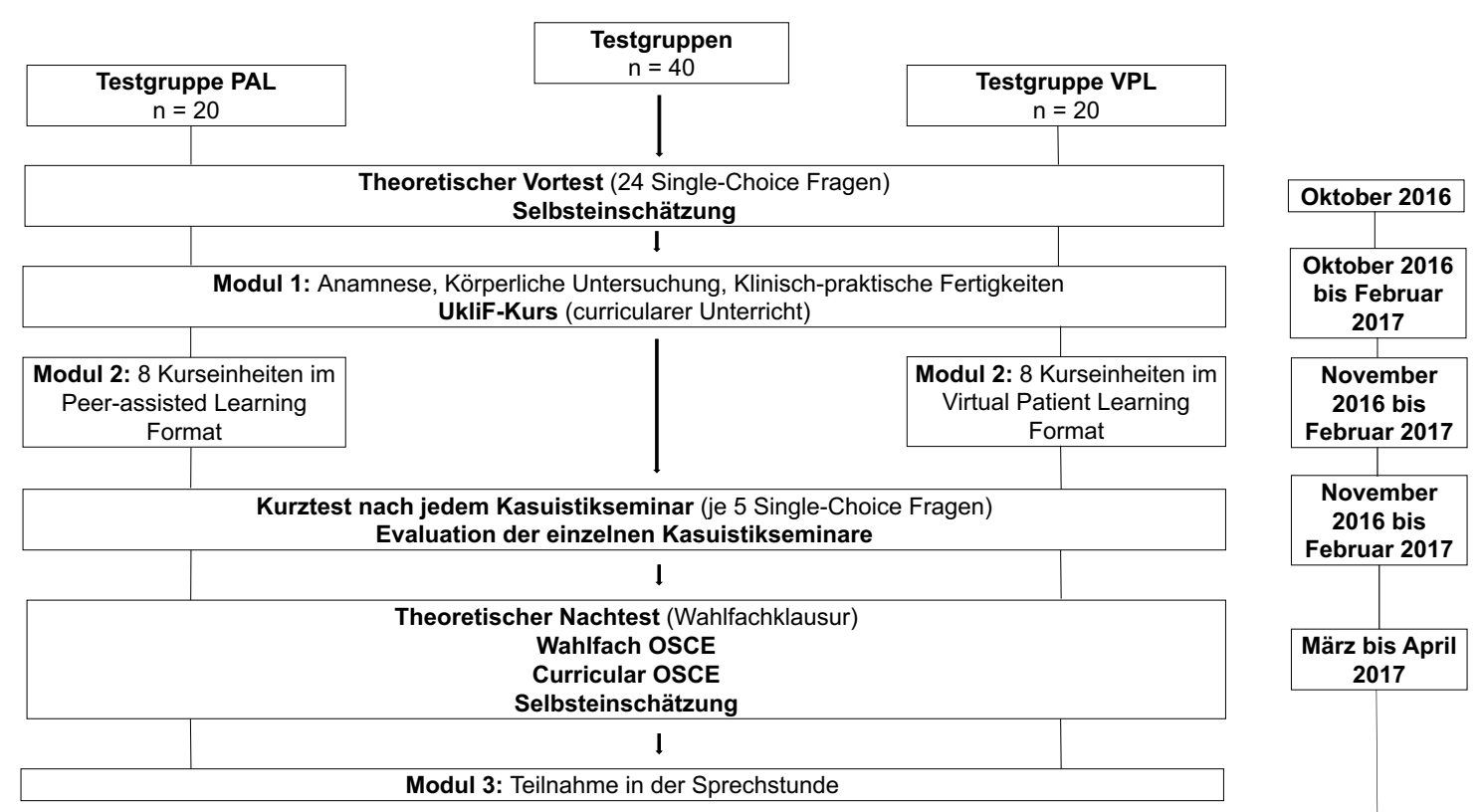

Abbildung 10: Überblick Wahlfach und Leistungsmessung mit Zeitverlauf

\subsubsection{Theoretische Tests}

\subsubsection{Theoretischer Langzeittest}

Vor Beginn der Wahlfachmodule absolvierten die Studenten beider Testgruppen und der Kontrollgruppe einen theoretischen Vortest zur Ermittlung des aktuellen Wissensstandes (Abb. 10). Dabei wurden 24 Single-Choice Fragen zu den Lernzielen der Kasuistikseminare gestellt. Jeweils drei Fragen bezogen sich auf eines der acht Themenkomplexe des Wahlfachmoduls 2. Diese 24 Single-Choice Fragen wurden im Vorausgang durch drei Experten (davon zwei Master of Medical Education) inhaltlich geprüft und in der vorangegangenen WahlfachKohorte getestet und validiert. Der Vortest wurde als Fragebogen mit dem Online Programm Google Forms durchgeführt. ${ }^{100}$ Für jede Frage wurde eine Bearbeitungszeit von 90 Sekunden vorgesehen. Die Gesamtzeit des Testes betrug 36 Minuten.

Nach Abschluss der ersten beiden Wahlfachmodule absolvierten die Studenten aller Gruppen erneut einen formativen theoretischen Test (Nachtest), der inhaltlich identisch zu dem Vortest war (Abb. 10). Sinn dieser Prüfung war, den langfristigen Lernerfolg und die Wissensretention über den Beobachtungszeitraum zu messen (Abb. 10). Der Nachtest fand in einem 
Seminarraum statt. Für jede Frage wurde eine Bearbeitungszeit von 90 Sekunden vorgegeben. Die Gesamtzeit betrug 36 Minuten. Die Fragen wurden in einer Power-Point Präsentation präsentiert und online mit Smartphones/ Tablets von den Studenten beantwortet.

\subsubsection{Theoretischer Kurzzeit-Test}

Neben der Langzeit-Wissensprüfung wurde auch das kurzzeitig erworbene Wissen getestet. Während des zweiten Wahlfachmoduls erfolgte unmittelbar nach jedem Fallseminar ein Kurztest zur jeweiligen Thematik, welcher fünf Single-Choice Fragen beinhaltete und sowohl in der PAL Gruppe als auch in der VPL Gruppe durchgeführt wurde (Abb. 10). Die Fragen wurden dem Fragenkatalog des IMPP entnommen. ${ }^{97}$ Die PAL Gruppe beantwortete die Fragen im Seminarraum, in welchem auch zuvor die Kasuistik stattfand. Die VPL Gruppe beantwortete die Fragen online und es erfolgte keine Zeitbegrenzung. Um gleiche Bedingungen für die PAL Gruppe zu schaffen, gab es auch in dieser Gruppe keine Zeitbegrenzung zur Beantwortung der Fragen. Zum Seminarthema Bauchschmerz wurde aufgrund von technischen Problemen kein Kurztest erhoben.

\subsubsection{Praktische Tests}

\subsubsection{Wahlfach OSCE}

Beide Testgruppen wurden zusätzlich zu der theoretischen Leistungsüberprüfung in einem OSCE in ihren praktischen Fertigkeiten geprüft (Abb. 10). Dieser Wahlfach OSCE lehnte sich an den curricularen OSCE an und beinhaltete insgesamt vier Stationen. Die Teilnehmer durchliefen zwei Untersuchungs-Stationen, eine Anamnese-Station und eine Feedback-Station.

\subsubsection{Curricularer OSCE}

Der curriculare OSCE der Studienteilnehmer wurde ebenfalls zur Leistungsmessung ausgewertet. Dieser OSCE beinhaltete insgesamt sechs Stationen (Abschnitt 6.2.4). 
In beiden OSCEs wurden die Studenten anhand vorher festgelegter Checklisten zur fachlichen Kompetenz (A-Note mit 70\%iger Wertung) und zur sozialen Kompetenz (B-Note mit 30\%iger Wertung) beurteilt.

\subsubsection{Selbsteinschätzung}

Darüber hinaus wurden die Studienteilnehmer gebeten vor und nach Absolvieren des Wahlfachs einen standardisierten Fragebogen zur Selbsteinschätzung ihrer Fähigkeiten im Bereich allgemeinmedizinischer Basiskompetenzen auszufüllen. Der Fragebogen beinhaltete 34 Fragen in den Bereichen „körperliche Untersuchung verschiedener Organsysteme“ (elf Fragen), „Kommunikation“ (sechs Fragen), „Klinisch-praktische Fertigkeiten“ (sieben Fragen), „Klinisches Denken“ (sechs Fragen) und „Therapie“ (vier Fragen) und lehnte sich inhaltlich an das Logbuch für Studenten im Praktischen Jahr aus dem Institut für Allgemeinmedizin der Goethe Universität Frankfurt an. ${ }^{101}$ Jede Frage wurde in Form einer sechs-stufigen Likert- Skala gestellt ( 1 = sehr sicher; 6 = überhaupt nicht sicher). Diese Selbsteinschätzung wurde vor und nach Durchlaufen aller drei Module erfasst. Die Selbstevaluation diente der subjektiven Einschätzung des Kompetenzerwerbs. Sie wurde als Online-Fragebogen mit Google Forms durchgeführt. ${ }^{100}$

\subsubsection{Seminarevaluation}

Nach jedem Seminar wurden die Studenten beider Testgruppen gebeten einen Evaluationsbogen mit je fünf Fragen in Form einer sechs-stufigen Likert-Skala zu beantworten ( 1 = ich stimme voll zu; 6 = ich stimme überhaupt nicht $z u$ ). Es wurden stets die gleichen fünf Fragen nach jedem Seminar gestellt:

1. "Ich bewerte die Kasuistik mit..."

2. "Ich habe viel lernen können in der Kasuistik."

3. „Ich konnte dem Fallbeispiel gut folgen."

4. "Ich weiß jetzt nicht mehr als vorher."

5. "Die Stoffmenge war angemessen." 
Die Studenten bewerteten damit die Seminare und konnten ihren persönlichen Lerneffekt angeben. Dieser Evaluationsbogen wurde ebenfalls mit Google Forms durchgeführt. 100

\subsection{Statistische Auswertung}

Für die statistische Auswertung und graphische Darstellung wurden Microsoft Office 2016 für Mac, Redmond Washington USA, ${ }^{96}$ und Graphpad Prism 7 für Mac, Graphpad Software, La Jolla California USA genutzt. ${ }^{102}$

Für die Prüfung auf signifikante Unterschiede dienten folgende Tests: WilcoxonTest (Wilcoxon signed-rank Test), Mann-Whitney-U-Test (Wilcoxon rank-sum Test) und Kruskal-Wallis-Test (Tab. 3).

Alle drei Tests gehören zur Gruppe von nicht-parametrischen Verfahren, die Unterschiede hinsichtlich der zentralen Tendenz oder des Mittelwerts zwischen zwei oder mehr Gruppen untersuchen. ${ }^{103}$

Das Signifikanzniveau wurde vor der Auswertung auf 5\% (0.05) festgelegt.

\begin{tabular}{|l|l|}
\hline $\begin{array}{l}\text { Wilcoxon-Test } \\
\text { (Vergleiche innerhalb } \\
\text { einer Gruppe) }\end{array}$ & $\begin{array}{l}\text { Vortest-Nachttest-Vergleich im theoretischen Langzeit- } \\
\text { Test für jeweils jede Gruppe }\end{array}$ \\
\cline { 2 - 2 } & $\begin{array}{l}\text { Vorher-Nachher-Vergleich in der Selbsteinschätzung für } \\
\text { jeweils jede Gruppe }\end{array}$ \\
\hline $\begin{array}{l}\text { Kruskal-Wallis-Test } \\
\text { (Vergleiche zwischen } \\
\text { mehr als zwei Gruppen) }\end{array}$ & $\begin{array}{l}\text { Gruppenvergleich PAL vs. VPL vs. KG für jeweils den } \\
\text { Vortest und Nachtest }\end{array}$ \\
\cline { 2 - 2 } $\begin{array}{l}\text { Mann-Whitney-U-Test } \\
\text { (Vergleiche zwischen zwei } \\
\text { Gruppen) }\end{array}$ & $\begin{array}{l}\text { Gruppenvergleich PAL vs. VPL vs. KG im curricularen } \\
\text { OSCE }\end{array}$ \\
\cline { 2 - 2 } & Kurzzeit-Test \\
\cline { 2 - 2 } & $\begin{array}{l}\text { Gruppenvergleich PAL vs. VPL im Wahlfach OSCE } \\
\text { Kasuistikseminare }\end{array}$ \\
\hline
\end{tabular}

Tabelle 3: Übersicht der für die Leistungsmessung genutzten Tests 


\subsubsection{Auswertung der theoretischen Tests}

\subsubsection{Auswertung des theoretischen Langzeit-Tests}

Zur Prüfung auf signifikante Unterschiede im Vortest zum Nachtest wurde für jeweils jede Gruppe (PAL, VPL und KG) der Wilcoxon-Test genutzt. Er diente zur Darstellung von signifikanten Unterschieden zwischen dem Vortest und Nachtest innerhalb der jeweiligen Gruppe. Der Wilcoxon-Test testet, ob die zentralen Tendenzen zweier abhängiger Stichproben verschieden sind. ${ }^{103}$ Abhängige Stichproben sind gegeben, wenn es eine Messwiederholung gibt. Die Messwerte stammen somit von der gleichen Person vor und nach Intervention. Folgende Fragestellungen wurden für den Test genannt: Erreichen Studenten nach Durchlaufen des VPL Seminars höhere Ergebnisse im Langzeit-Wissenserwerb? Erreichen Studenten nach Durchlaufen des PAL Seminars höhere Ergebnisse im Langzeit-Wissenserwerb?

Für die Prüfung signifikanter Unterschiede zwischen der PAL Gruppe, VPL Gruppe und KG wurde der Kruskal-Wallis-Test jeweils für den Vortest und Nachtest genutzt. Der Kruskal-Wallis-Test testet, ob sich zentrale Tendenzen mehrerer unabhängiger Stichproben unterscheiden, ${ }^{103}$ er diente zum Vergleich der Gruppen untereinander. In der vorliegenden Arbeit wurde er für folgende Fragestellung herangezogen:

Unterscheiden sich PAL, VPL und KG bezüglich ihres allgemeinmedizinischen Wissens (jeweils im Vortest und Nachtest)?

\subsubsection{Auswertung des theoretischen Kurzzeit-Tests}

Zur Prüfung signifikanter Unterschiede zwischen PAL Gruppe und VPL Gruppe im theoretischen Kurzzeit-Test wurde der Mann-Whitney-U-Test genutzt. Dieser testet, ob die zentralen Tendenzen zweier unabhängiger Stichproben verschieden sind. ${ }^{103}$ Folgende Fragestellung veranschaulicht den Test: Zeigen Studenten der PAL Gruppe höhere Ergebnisse im kurzzeitigen Wissenserwerb als die VPL Gruppe? 


\subsubsection{Auswertung der praktischen Tests}

\subsubsection{Auswertung des Wahlfach OSCEs}

Für die Prüfung auf signifikante Unterschiede zwischen der PAL Gruppe und VPL Gruppe im Wahlfach OSCE wurde der Mann-Whitney-U-Test genutzt. Folgende Fragestellung veranschaulicht den Test: Zeigen Studenten der PAL Gruppe höhere Ergebnisse in praktischen Fertigkeiten als die VPL Gruppe?

\subsubsection{Auswertung des curricularen OSCEs}

Für die Auswertung des curricularen OSCES und den Vergleich aller drei Gruppen (PAL, VPL, KG) wurde der Kruskal-Wallis-Test genutzt. Folgende Fragestellung veranschaulicht den Test: Unterscheiden sich PAL, VPL und KG (drei Gruppen) bezüglich ihrer praktischen Fertigkeiten im curricularen OSCE?

\subsubsection{Auswertung der Selbsteinschätzung}

Für die Prüfung auf signifikante Unterschiede in der Selbsteinschätzung wurde für jeweils jede Gruppe (PAL, VPL und KG) der Wilcoxon-Test genutzt. Dieser Test diente der Auswertung des subjektiven Kompetenzerwerbs innerhalb jeweils einer Gruppe. Folgende Fragen stellte sich im Test: Schätzen sich Studenten nach Durchlaufen des VPL Seminars besser ein hinsichtlich allgemeinmedizinischer Grundfertigkeiten als vorher? Schätzen sich Studenten nach Durchlaufen des PAL Seminars besser ein hinsichtlich allgemeinmedizinischer Grundfertigkeiten als vorher?

\subsubsection{Auswertung der Seminarevaluation}

Für die Prüfung auf signifikante Unterschiede zwischen den beiden (PAL, VPL) Testgruppen wurde in der Evaluation der einzelnen Kasuistikseminare der MannWhitney-U-Test genutzt. Folgende Fragestellung veranschaulicht den Test: Zeigen sich Unterschiede in der Bewertung der Kasuistikseminare? 


\section{ERGEBNISSE}

\subsection{Demographische Daten der Studienteilnehmer}

Die PAL Gruppe setzte sich zusammen aus 17 weiblichen und drei männlichen Teilnehmern. Sechs der 20 Gruppenmitglieder (30\%) hatten bereits in einem medizinischen Beruf gearbeitet. Der Großteil der PAL Gruppe war im Alter von 21-23 Jahren (70\%) (Tab. 4). Die VPL Gruppe setzte sich zusammen aus 14 weiblichen und sechs männlichen Teilnehmern. Fünf der 20 Gruppenmitglieder (25\%) hatten bereits in einem medizinischen Beruf gearbeitet. Mit 65\% (13 Teilnehmer von 20) zeigte auch die VPL Gruppe den Großteil im Alter von 21-23 Jahren (Tab. 4). Die KG $(n=11)$ setzte sich nur aus weiblichen Teilnehmern zusammen. Auch in dieser Gruppe war der Großteil 21-23 Jahre alt (64\%, Tab. 4). Einer der 11 Gruppenmitglieder hatte bereits in einem medizinischen Beruf gearbeitet.

\begin{tabular}{|l|c|c|c|}
\hline Altersspanne (Jahre) & PAL & VPL & KG \\
\hline $\mathbf{1 8 - 2 1}$ & $20 \%$ & $15 \%$ & $27 \%$ \\
\hline $\mathbf{2 1 - 2 3}$ & $70 \%$ & $65 \%$ & $64 \%$ \\
\hline $\mathbf{2 4 - 2 6}$ & $0 \%$ & $10 \%$ & $9 \%$ \\
\hline $\mathbf{2 7 - 2 9}$ & $5 \%$ & $5 \%$ & $0 \%$ \\
\hline Über 30 & $5 \%$ & $5 \%$ & $0 \%$ \\
\hline
\end{tabular}

Tabelle 4: Altersverteilung der Studiengruppen

\subsection{Ergebnisse der theoretischen Tests}

\subsubsection{Ergebnisse des theoretischen Langzeit-Tests}

48 von 51 Studenten $(P A L=20$, Antwortrate $=100 \% ; \mathrm{VPL}=20$, Antwortrate $=$ $100 \% ; \mathrm{KG}=8$; Antwortrate $=72,7 \%$ ) absolvierten den theoretischen Vortest. Der Nachtest wurde von 47 Studenten absolviert $(P A L=20$. Antwortrate $=100 \%$; VPL $=20$. Antwortrate $=100 \% ; K G=7$, Antwortrate $=63,6 \%$ ).

Es zeigte sich in beiden Testgruppen ein signifikanter Wissenszuwachs im Nachtest im Vergleich zum Vortest (Tab. 5, Abb. 11). Beide Gruppen erzielten einen $\mathrm{p}$-Wert von $<0.0001$ für den gesamten Test (Tab. 5).

In der nach Themenbereichen durchgeführten Item Analyse des theoretischen Langzeit-Tests erzielte die PAL Gruppe in sieben von acht Items einen 
signifikanten Wissenszuwachs, während die VPL Gruppe in sechs von acht Themen einen signifikanten Wissenszuwachs erzielte (Tab. 5).

Die KG erzielte im theoretischen Langzeit-Test ebenfalls einen signifikanten Unterschied ( $p=0.0156$; Tab. 5). Betrachtet man die Item Analyse des theoretischen Langzeit-Tests, konnte die KG jedoch nur in einem der acht Items einen signifikanten Unterschied zwischen Vortest und Nachtest erzielen (Thema „Bauchschmerz“, $p=0.0313)$. Der Vergleich des Vortestes zwischen allen drei Studiengruppen zeigte keinen signifikanten Unterschied $(p=0.1207 ;$ Tab. 6, Abb. 11). Alle Studienteilnehmer präsentierten sich somit zu Beginn der Studie mit einem ähnlichen Wissensstand. In der vergleichenden Analyse aller Studiengruppen im Nachtest war ein signifikanter Unterschied auszumachen ( $p$ < 0.0001; Tab. 6, Abb. 11). Die PAL Gruppe erzielte einen Mittelwert von 95,62\%, die VPL Gruppe einen Mittelwert von 94,17\% im Nachtest (Tab. 5). Die KG erreichte insgesamt einen Mittelwert von 65,48\% im Nachtest (Tab. 5).

\begin{tabular}{|l|c|c|c|c|c|c|c|c|c|}
\hline Item & $\begin{array}{c}\text { PAL } \\
\text { vor } \\
(\%)\end{array}$ & $\begin{array}{c}\text { PAL } \\
\text { nach } \\
(\%)\end{array}$ & $\begin{array}{c}\text { PAL } \\
\mathbf{p}-\text { Wert }\end{array}$ & $\begin{array}{c}\text { VPL } \\
\text { vor } \\
(\%)\end{array}$ & $\begin{array}{c}\text { VPL } \\
\text { nach } \\
(\%)\end{array}$ & $\begin{array}{c}\text { VPL } \\
\mathbf{p} \text { - Wert }\end{array}$ & $\begin{array}{c}\text { KG } \\
\text { vor } \\
(\%)\end{array}$ & $\begin{array}{c}\text { KG } \\
\text { nach } \\
(\%)\end{array}$ & $\begin{array}{c}\text { KG } \\
\mathbf{p} \text { - Wert }\end{array}$ \\
\hline $\begin{array}{l}\text { Gesamter } \\
\text { Test }\end{array}$ & 58.75 & 95.62 & $<0.0001$ & 53.55 & 94.17 & $<0.0001$ & 53,65 & 65.48 & 0.0156 \\
\hline $\begin{array}{l}\text { Bauch- } \\
\text { schmerz }\end{array}$ & 20.00 & 96.67 & $<0.0001$ & 25.56 & 90.00 & $<0.0001$ & 4.76 & 33.33 & 0.0313 \\
\hline Hypertonie & 91.67 & 96.67 & 0.4531 & 79.44 & 98.33 & 0.1250 & 76.19 & 100.00 & 0.1250 \\
\hline $\begin{array}{l}\text { Husten } \\
\text { Kreuz- } \\
\text { schmerz }\end{array}$ & 46.67 & 93.33 & $<0.0001$ & 37.78 & 98.33 & $<0.0001$ & 47.62 & 76.19 & 0.1250 \\
\hline Dyspnoe & 66.67 & 100.00 & 0.0001 & 71.11 & 100.00 & 0.0005 & 57.14 & 66.67 & 0.5000 \\
\hline $\begin{array}{l}\text { Schwanger } \\
\text {-schaft }\end{array}$ & 60.00 & 85.00 & 0.0029 & 32.78 & 86.67 & $<0.0001$ & 52.38 & 52.38 & $>0.9999$ \\
\hline $\begin{array}{l}\text { Kopf- } \\
\text { schmerz }\end{array}$ & 48.33 & 100.00 & $<0.0001$ & 24.44 & 88.33 & $<0.0001$ & 42.86 & 38.10 & 0.5000 \\
\hline Polyurie & 85.00 & 98.33 & 0.0156 & 71.67 & 95.00 & 0.0859 & 90.48 & 95.24 & $>0.9999$ \\
\hline
\end{tabular}

Tabelle 5: Vergleich von Vortest und Nachtest in den einzelnen Studiengruppen (PAL, VPL und KG) nach erfolgter thematischer Unterteilung und Item Analyse der bearbeiteten Testfragen 


\begin{tabular}{|l|c|}
\hline Miteinander verglichene Studiengruppe & p-Wert \\
\hline $\begin{array}{l}\text { Vortest PAL vs. VPL vs. KG } \\
\text { (Kruskal- Wallis- Test) }\end{array}$ & 0.1207 \\
\hline $\begin{array}{l}\text { Nachtest PAL vs. VPL vs. KG } \\
\text { (Kruskal- Wallis- Test) }\end{array}$ & $<0.0001$ \\
\hline
\end{tabular}

Tabelle 6: Vergleich von PAL, VPL und KG untereinander im Vortest und Nachtest
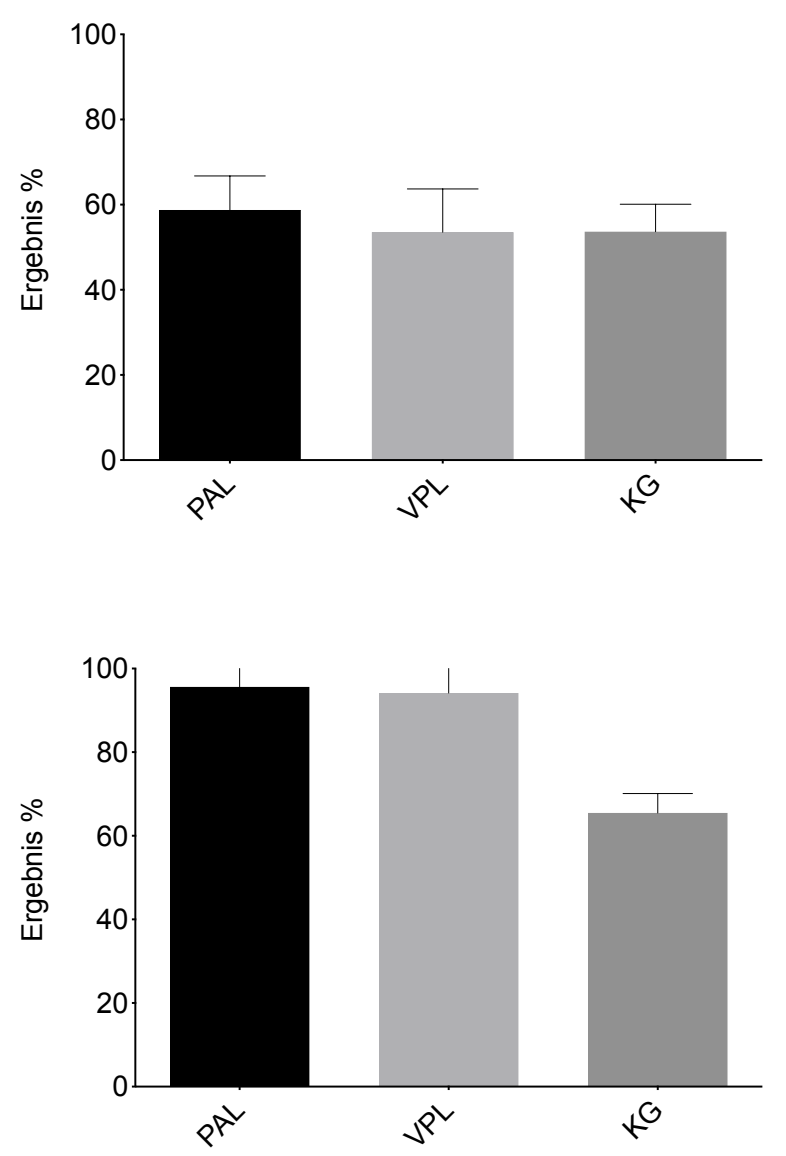

Abbildung 11: Vergleich aller Studiengruppen im Vortest (links) und Nachtest (rechts)

\subsubsection{Ergebnisse des theoretischen Kurzzeit-Tests}

$\mathrm{Zu}$ sieben der acht Kasuistik Seminar-Themen wurden Kurztests durchgeführt. 40 von 40 möglichen Studenten nahmen teil $(P A L=20$, Antwortrate $=100 \%$ VPL $=20$, Antwortrate $=100 \%$ ).

Betrachtet man alle Kurztests gemeinsam, so wies die VPL Gruppe ein signifikant besseres Ergebnis auf als die PAL Gruppe (Mittelwert PAL $=85.75 \%$; Mittelwert VPL $=90.57 \% ; p=0.0047 ;$ Tab. 7, Abb. 12). 
In der vergleichenden Analyse der einzelnen Kurztests miteinander, erzielte die VPL Gruppe in fünf von sieben Fällen prozentual ein besseres Ergebnis als die PAL Gruppe (Tab. 7). Jedoch zeigte sich nur in einem der Fälle ein signifikant besseres Ergebnis zugunsten der VPL Gruppe (Kasuistik „Belastungsdyspnoe und Leistungsminderung: $p=0.0116)$.

\begin{tabular}{|l|c|c|c|}
\hline Item & Mittelwert PAL (\%) & Mittelwert VPL (\%) & P-Wert \\
\hline Gesamter Test & 85.75 & 90.57 & 0.0047 \\
\hline Hypertonie & 88.00 & 87.00 & 0.8060 \\
\hline Husten & 82.00 & 86.00 & 0.6032 \\
\hline Kreuzschmerz & 87.00 & 86.00 & 0.8889 \\
\hline Dyspnoe & 81.00 & 94.00 & 0.0116 \\
\hline Schwangerschaft & 91.00 & 96.00 & 0.1760 \\
\hline Kopfschmerz & 85.00 & 92.00 & 0.2121 \\
\hline Polyurie & 86.32 & 93.00 & 0.1394 \\
\hline
\end{tabular}

Tabelle 7: Vergleich der Kurztestergebnisse zwischen PAL und VPL; zur Kasuistik Bauchschmerz wurde kein Kurztest durchgeführt

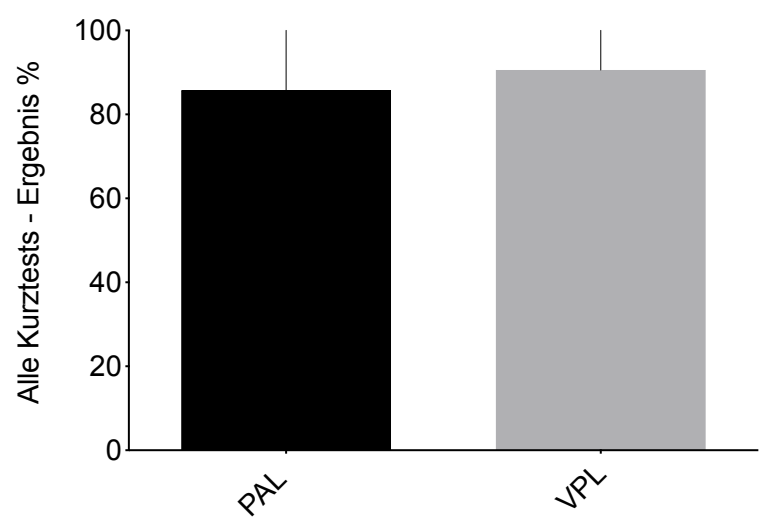

Abbildung 12: Ergebnis aller Kurztests in PAL und VPL 


\subsection{Ergebnisse der praktischen Tests}

\subsubsection{Ergebnisse des Wahlfach OSCEs}

Am Wahlfach OSCE nahmen 39 von 40 Studenten teil (PAL $n=20$, Antwortrate $=100 \% ;$ VPL $n=19 ;$ Antwortrate $=95 \%$ ).

Im Vergleich ergaben sich keine signifikanten Leistungsunterschiede zwischen der PAL Gruppe und VPL Gruppe ( $p=0.5395 ;$ Tab. 8; Abb. 13). Die Item Analyse der einzelnen Prüfungsstationen ergab ebenfalls keinen signifikanten Unterschied zwischen den beiden Testgruppen (Tab. 8). Die PAL Gruppe erreichte durchschnittlich 80,26\% und die VPL Gruppe 79,30\% (Tab. 8).

\begin{tabular}{|l|c|c|c|c|c|}
\hline Station & $\begin{array}{c}\text { PAL } \\
\text { Mittelwert } \\
\%\end{array}$ & $\begin{array}{c}\text { PAL } \\
\text { Median } \\
\text { Note }\end{array}$ & $\begin{array}{c}\text { VPL } \\
\text { Mittelwert } \\
\%\end{array}$ & $\begin{array}{c}\text { VPL } \\
\text { Median } \\
\text { Note }\end{array}$ & p-Wert \\
\hline Anamnese & 86.76 & 2 & 84.30 & 2 & 0.1545 \\
\hline Feedback & 69.50 & 5 & 72.11 & 5 & 0.6420 \\
\hline Untersuchung Abdomen & 90.23 & 1 & 90.39 & 2 & 0.8709 \\
\hline $\begin{array}{l}\text { Untersuchung Kardiologie } \\
\text { oder Pulmologie }\end{array}$ & 91.02 & 1.5 & 70.38 & 3 & 0.346 \\
\hline Gesamt & 80.26 & 2 & 79.30 & 2 & 0.5395 \\
\hline
\end{tabular}

Tabelle 8: Ergebnisse im Wahlfach OSCE

\subsubsection{Ergebnisse des curricularen OSCEs}

Am curricularen OSCE beteiligten sich 50 von 51 Studenten (PAL $n=20$, Antwortrate $=100 \% ;$ VPL $n=19$, Antwortrate $=95 \% ; K G n=11$, Antwortrate $=$ $100 \%$ ). Es zeigte sich im gesamten OSCE kein signifikanter Unterschied zwischen beiden Testgruppen und der Kontrollgruppe ( $p=0.4263$; Tab. 9; Abb. 13). Die PAL Gruppe erreichte einen Mittelwert von $82,6 \%$, die VPL Gruppe $81,44 \%$ und die KG $85,06 \%$ (Tab. 9).

Die Einzelanalyse der untersuchten OSCE-Stationen ergab in einer von sechs Stationen einen signifikanten Unterschied zwischen beiden Testgruppen und der Kontrollgruppe. Nur in der Station „Untersuchung Kardiologie/ Angiologie/ Pulmologie" zeigte die Kontrollgruppe eine signifikant bessere Leistung $(p=$ 0.0322; Tab. 9). 


\begin{tabular}{|l|c|c|c|c|}
\hline Station & $\begin{array}{c}\text { PAL } \\
\text { Mittelwert } \\
\%\end{array}$ & $\begin{array}{c}\text { VPL } \\
\text { Mittelwert } \\
\%\end{array}$ & $\begin{array}{c}\text { KG } \\
\text { Mittelwert } \\
\%\end{array}$ & p-Wert \\
\hline Gesamt & 82.6 & 81.44 & 85.06 & 0.4263 \\
\hline Anamnese & 83.45 & 81.29 & 81.92 & 0.5574 \\
\hline $\begin{array}{l}\text { Untersuchung } \\
\text { (Gastroenterologie oder } \\
\text { Hämatologie) }\end{array}$ & 88.34 & 85.75 & 87.29 & 0.8201 \\
\hline $\begin{array}{l}\text { Untersuchung (Kardiologie, } \\
\text { Angiologie oder Pulmologie) }\end{array}$ & 81.12 & 85.95 & 92.69 & 0.0322 \\
\hline Untersuchung Neurologie & 88.00 & 79.83 & 89.44 & 0.1233 \\
\hline Patientenvorstellung & 91.58 & 89.77 & 91.82 & 0.3741 \\
\hline Flurstation & 63.14 & 66.05 & 67.21 & 0.7941 \\
\hline
\end{tabular}

Tabelle 9: Ergebnisse im Curricularen OSCE

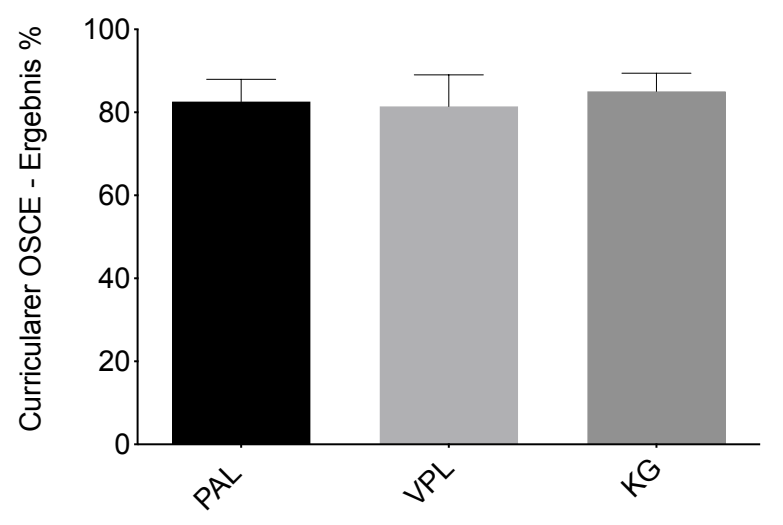

Abbildung 13: Ergebnisse im Curricularen OSCE

\subsection{Ergebnisse der Selbsteinschätzung}

An der Selbsteinschätzung nahmen vor Studiendurchführung 51 von 51 Studenten teil (PAL $n=20$, Antwortrate $=100 \%$; VPL $n=20$, Antwortrate $=100 \%$; $K G n=11$, Antwortrate $=100 \%$ ). Nach Abschluss des Wahlfachs nahmen 43 von 51 Studenten an der Selbsteinschätzung teil $(P A L n=20$, Antwortrate $=100 \%$; VPL $n=15$, Antwortrate $=75 \% ; K G n=8 ;$ Antwortrate $=72,7 \%$ ).

In 31 von 34 untersuchten Items schätze sich die PAL Gruppe signifikant besser ein als vorher (Tab. 10). Die VPL Gruppe schätze sich in 25 der 34 untersuchten 
Items signifikant besser ein als zuvor (Tab. 10). Die KG gab an, sich in 16 der 34 Items signifikant sicherer zu fühlen (Tab. 10).

In den elf untersuchten Fragen zur Kompetenz in der körperlichen Untersuchung zeigten sich in allen drei Gruppen ähnliche Ergebnisse (Tab. 10): Die PAL Gruppe zeigte in elf von elf Items, die VPL Gruppe in neun von elf und die KG in zehn von elf Items ein signifikant besseres Ergebnis (Tab. 10). Im Bereich Kommunikation wies die PAL Gruppe in fünf von sechs und die VPL Gruppe in drei von sechs Items eine signifikant bessere Selbsteinschätzung auf (Tab. 10). Die KG hingegen konnte sich nur in einem Bereich der Kommunikation signifikant verbessern (Tab. 10).

In den klinisch-praktischen Fertigkeiten schätze sich die PAL Gruppe in fünf von sieben und die VPL Gruppe in vier von sieben Items signifikant besser ein als zuvor (Tab. 10). Die KG schätze sich in diesem Bereich in keinem der ltems sicherer ein als vor Studienbeginn (Tab. 10).

Im Bereich Klinisches Denken schätze sich die PAL Gruppe in sechs von sechs Items signifikant besser ein (Tab. 10). Die VPL Gruppe schätzte sich in fünf Items und die KG in drei der sechs Items signifikant besser ein (Tab. 10).

Im Bereich Therapie zeigten sowohl die PAL Gruppe als auch die VPL Gruppe in vier der vier gefragten Items eine signifikant bessere Selbsteinschätzung nach Abschluss des Wahlfachs (Tab. 10). Die KG hingegen schätzte sich nur in zwei der vier Items signifikant besser ein (Tab. 10).

\begin{tabular}{|l|c|c|c|c|c|c|c|c|c|}
\hline Untersuchung & $\begin{array}{c}\text { PAL } \\
\text { vor }\end{array}$ & $\begin{array}{c}\text { PAL } \\
\text { nach }\end{array}$ & p-Wert & $\begin{array}{c}\text { VPL } \\
\text { vor }\end{array}$ & $\begin{array}{c}\text { VPL } \\
\text { nach }\end{array}$ & p-Wert & $\begin{array}{c}\text { KG } \\
\text { vor }\end{array}$ & $\begin{array}{c}\text { KG } \\
\text { nach }\end{array}$ & p-Wert \\
\hline Herz-Kreislauf & 4,25 & 1,75 & $<0.0001$ & 4,3 & 1,93 & 0.0002 & 4,73 & 2,38 & 0.0078 \\
\hline Abdomen & 4,65 & 1,6 & $<0.0001$ & 5,25 & 1,93 & $<0.0001$ & 5,45 & 2,38 & 0.0078 \\
\hline Lunge & 4,35 & 1,8 & $<0.0001$ & 4,5 & 1,87 & 0.0006 & 4,45 & 1,63 & 0.0078 \\
\hline Bewegungsapparat & 4,3 & 2,9 & 0.0009 & 4,6 & 3,4 & 0.0454 & 4,73 & 3,5 & 0.0156 \\
\hline Gefäße & 4,5 & 2,75 & 0.0006 & 4,8 & 2,53 & 0.0017 & 5,27 & 2,13 & 0.0078 \\
\hline $\begin{array}{l}\text { Lymphatische } \\
\text { Organe }\end{array}$ & 4,85 & 2,05 & $<0.0001$ & 4,9 & 2,47 & 0.0004 & 5,18 & 2,25 & 0.0078 \\
\hline Haut & 4,3 & 3,1 & 0.0331 & 4,5 & 3,6 & 0.2129 & 4,91 & 3,38 & 0.0078 \\
\hline Nervensystem & 3,5 & 2,45 & 0.0083 & 3,75 & 2,47 & 0.0674 & 3,91 & 1,75 & 0.0078 \\
\hline Augen/HNO & 4,25 & 3,05 & 0.0015 & 4,7 & 2,8 & 0.0010 & 4,91 & 2,88 & 0.0078 \\
\hline $\begin{array}{l}\text { Krebsvorsorge } \\
\text { Schwangerschafts- } \\
\text { Untersuchung }\end{array}$ & 5,05 & 3,35 & 0.0004 & 5,6 & 3,87 & 0.0024 & 5,64 & 4,38 & 0.0078 \\
\hline
\end{tabular}

Tabelle 10a: Selbsteinschätzung vorher und nachher in der PAL Gruppe, VPL Gruppe und KG - Untersuchung 


\begin{tabular}{|l|c|c|c|c|c|c|c|c|c|}
\hline Kommunikation & $\begin{array}{c}\text { PAL } \\
\text { vor }\end{array}$ & $\begin{array}{c}\text { PAL } \\
\text { nach }\end{array}$ & p-Wert & $\begin{array}{c}\text { VPL } \\
\text { vor }\end{array}$ & $\begin{array}{c}\text { VPL } \\
\text { nach }\end{array}$ & p-Wert & $\begin{array}{c}\text { KG } \\
\text { vor }\end{array}$ & $\begin{array}{c}\text { KG } \\
\text { nach }\end{array}$ & p-Wert \\
\hline $\begin{array}{l}\text { Anamnese- } \\
\text { Erhebung }\end{array}$ & 3,15 & 1,7 & 0.0004 & 3,1 & 1,53 & 0.0009 & 3,09 & 2,25 & 0.2500 \\
\hline $\begin{array}{l}\text { Psychische } \\
\text { Ursachen für } \\
\text { Erkrankungen } \\
\text { erkennen }\end{array}$ & 3,25 & 2,65 & 0.1808 & 3,75 & 2,87 & 0.0776 & 4,36 & 5,38 & 0.1563 \\
\hline $\begin{array}{l}\text { Gesprächsführung } \\
\text { mit Patienten }\end{array}$ & 2,25 & 1,5 & 0.0090 & 2,4 & 1,53 & 0.0972 & 2,45 & 1,88 & 0.2188 \\
\hline $\begin{array}{l}\text { Diätberatung } \\
\text { Impfberatung }\end{array}$ & 4,7 & 3,4 & 0.0017 & 4,6 & 2,8 & 0.0029 & 5,27 & 4,25 & 0.0938 \\
\hline $\begin{array}{l}\text { Überbringen } \\
\text { schlechter } \\
\text { Nachrichten }\end{array}$ & 3,7 & 2,65 & 0.0288 & 3,9 & 3,6 & 0.5156 & 3,91 & 3,25 & 0.2656 \\
\hline
\end{tabular}

Tabelle 10b: Selbsteinschätzung vorher und nachher in der PAL Gruppe, VPL Gruppe und KG - Kommunikation

\begin{tabular}{|l|c|c|c|c|c|c|c|c|c|}
\hline $\begin{array}{l}\text { Klinisch- } \\
\text { praktische } \\
\text { Fertigkeiten }\end{array}$ & $\begin{array}{l}\text { PAL } \\
\text { vor }\end{array}$ & $\begin{array}{c}\text { PAL } \\
\text { nach }\end{array}$ & p-Wert & $\begin{array}{l}\text { VPL } \\
\text { vor }\end{array}$ & $\begin{array}{l}\text { VPL } \\
\text { nach }\end{array}$ & p-Wert & $\begin{array}{c}\text { KG } \\
\text { vor }\end{array}$ & $\begin{array}{c}\text { KG } \\
\text { nach }\end{array}$ & p-Wert \\
\hline Blutdruckmessung & 1,15 & 1,15 & $>0.9999$ & 1,5 & 1,07 & 0.1094 & 1,55 & 1,25 & 0.3750 \\
\hline $\begin{array}{l}\text { EKG (Schreiben, } \\
\text { Befunden) }\end{array}$ & 4,1 & 2,95 & 0.0023 & 3,95 & 2,67 & 0.0232 & 3,91 & 3,0 & 0.0625 \\
\hline $\begin{array}{l}\text { Abdominelle } \\
\text { Sonographie }\end{array}$ & 5,35 & 3,1 & $<0.0001$ & 5,3 & 3,47 & 0.0009 & 5,27 & 4,63 & 0.0625 \\
\hline $\begin{array}{l}\text { Blutentnahme/ } \\
\text { Venenverweilkanüle }\end{array}$ & 4,85 & 2,75 & 0.0002 & 4,75 & 3,2 & 0.0963 & 5,18 & 4,75 & 0.7500 \\
\hline $\begin{array}{l}\text { I.m.-Injektion und } \\
\text { Impfung }\end{array}$ & 3,85 & 3,25 & 0.2007 & 4,75 & 3,4 & 0.0479 & 4,09 & 3,88 & 0.3750 \\
\hline $\begin{array}{l}\text { Urin-Stix } \\
\text { Hygienisches } \\
\text { Arbeiten }\end{array}$ & 3,4 & 2,25 & 0.0312 & 4,25 & 1,67 & 0.0022 & 4,0 & 3,38 & 0.2188 \\
\hline
\end{tabular}

Tabelle 10c: Selbsteinschätzung vorher und nachher in der PAL Gruppe, VPL Gruppe und KG - Klinisch-praktische Fertigkeiten 


\begin{tabular}{|l|c|c|c|c|c|c|c|c|c|}
\hline Klinisches Denken & $\begin{array}{l}\text { PAL } \\
\text { vor }\end{array}$ & $\begin{array}{c}\text { PAL } \\
\text { nach }\end{array}$ & p-Wert & $\begin{array}{c}\text { VPL } \\
\text { vor }\end{array}$ & $\begin{array}{c}\text { VPL } \\
\text { nach }\end{array}$ & p-Wert & $\begin{array}{c}\text { KG } \\
\text { vor }\end{array}$ & $\begin{array}{c}\text { KG } \\
\text { nach }\end{array}$ & p-Wert \\
\hline $\begin{array}{l}\text { Einleitung korrekter } \\
\text { Diagnostik }\end{array}$ & 4,9 & 2,65 & $<0.0001$ & 4,8 & 2,73 & 0.0002 & 5,09 & 3,63 & 0.0156 \\
\hline $\begin{array}{l}\text { Interpretation } \\
\text { Laborparameter }\end{array}$ & 4,6 & 2,65 & $<0.0001$ & 4,65 & 2,6 & 0.0013 & 4,91 & 2,75 & 0.0156 \\
\hline $\begin{array}{l}\text { Therapieplan- } \\
\text { Erstellung }\end{array}$ & 5,4 & 3,2 & $<0.0001$ & 5,5 & 3,27 & 0.0002 & 5,45 & 4,63 & 0.0625 \\
\hline $\begin{array}{l}\text { Differentialdiagnosen } \\
\text { abwägen }\end{array}$ & 4,95 & 2,75 & $<0.0001$ & 5,2 & 2,87 & 0.0010 & 5,55 & 3,75 & 0.0156 \\
\hline $\begin{array}{l}\text { Organisation einer } \\
\text { Patientenversorgung }\end{array}$ & 4,15 & 3,05 & 0.0411 & 4,75 & 3,73 & 0.2573 & 4,55 & 3,5 & 0.0859 \\
\hline Mutterpass ausstellen & 5,2 & 3,8 & 0.0083 & 5,9 & 3,87 & 0.0007 & 5,55 & 5,38 & 0.1250 \\
\hline
\end{tabular}

Tabelle 10d: Selbsteinschätzung vorher und nachher in der PAL Gruppe, VPL Gruppe und KG - Klinisches Denken

\begin{tabular}{|l|c|c|c|c|c|c|c|c|c|}
\hline Therapie & $\begin{array}{c}\text { PAL } \\
\text { vor }\end{array}$ & $\begin{array}{c}\text { PAL } \\
\text { nach }\end{array}$ & p-Wert & $\begin{array}{c}\text { VPL } \\
\text { vor }\end{array}$ & $\begin{array}{c}\text { VPL } \\
\text { nach }\end{array}$ & $\begin{array}{c}\text { p- } \\
\text { Wert }\end{array}$ & $\begin{array}{c}\text { KG } \\
\text { vor }\end{array}$ & $\begin{array}{c}\text { KG } \\
\text { nach }\end{array}$ & $\begin{array}{c}\text { p- } \\
\text { Wert }\end{array}$ \\
\hline $\begin{array}{l}\text { Bluthochdruck } \\
\text { medikamentös } \\
\text { einstellen }\end{array}$ & 5,3 & 2,65 & $<0.0001$ & 5,65 & 2,8 & 0.0001 & 5,64 & 4,5 & 0.0313 \\
\hline $\begin{array}{l}\text { Diabetes } \\
\text { medikamentös } \\
\text { einstellen }\end{array}$ & 4,85 & 2,6 & $<0.0001$ & 5,25 & 2,8 & 0.0010 & 5,55 & 4,75 & 0.1250 \\
\hline $\begin{array}{l}\text { Kreuzschmerzen } \\
\text { leitliniengerecht } \\
\text { therapieren }\end{array}$ & 4,95 & 3,1 & $<0.0001$ & 5,7 & 3,07 & 0.0002 & 5,82 & 5,5 & 0.2500 \\
\hline $\begin{array}{l}\text { Tuberkulose } \\
\text { Management } \\
\text { (Diagnostik und } \\
\text { Therapie) }\end{array}$ & 5,45 & 3,05 & $<0.0001$ & 5,6 & 3,2 & 0.0066 & 5,73 & 4,75 & 0.0313 \\
\hline
\end{tabular}

Tabelle 10e: Selbsteinschätzung vorher und nachher in der PAL Gruppe, VPL Gruppe und KG - Therapie

\subsection{Ergebnisse der Seminarevaluation}

An den einzelnen Evaluationen zu den Kasuistikseminaren nahmen 40 von 40 Studenten teil $(\mathrm{PAL} n=20$, Antwortrate $=100 \%$; VPL $\mathrm{n}=20$, Antwortrate $=$ $100 \%)$.

Zu jeder Kasuistik wurden folgende fünf Fragen beantwortet

1. "Ich bewerte die Kasuistik mit..."

In keiner der acht Kasuistiken zeigte sich ein signifikanter Unterschied (Tab.

11). Beide Testgruppen bewerteten alle Kasuistiken im Median mit der Schulnote 1 oder 2 (Tab. 11). 
2. „Ich habe viel lernen können in der Kasuistik.“

In sieben von acht Kasuistiken zeigte sich kein signifikanter Unterschied zwischen beiden Testgruppen. Nur in der Kasuistik „Schwangerschaft“ schätzte die VPL Gruppe ihren Wissenszuwachs signifikanten höher ein ( $p=$ 0.0254; Tab. 11). Auch hier wurden die Kasuistiken mit dem Median 1 oder 2 bewertet (Tab. 11).

3. „Ich konnte dem Fallbeispiel gut folgen.“

Auch hier zeigte sich in sieben von acht Kasuistiken kein signifikanter Unterschied zwischen beiden Testgruppen, mit Ausnahme der Kasuistik „Schwangerschaft“ in der erneut ein signifikanter Unterschied zugunsten der VPL Gruppe gemessen werden konnte (PAL Median = 1; VPL Median = 2; $p$ = 0.0076; Tab. 11).

4. "Ich weiß nicht viel mehr als vorher."

In fünf von acht Kasuistiken war kein signifikanter Unterschied zu messen. Die drei Kasuistiken „Kreuzschmerz“, „Dyspnoe und Leistungsminderung“ und „Polyurie“ wiesen einen signifikanten Unterschied zugunsten der PALGruppe auf.

5. "Die Menge an Lehrinhalt war angemessen."

In allen acht Kasuistiken war kein signifikanter Unterschied zu messen. Die Frage wurde in den Kasuistiken im Median mit einer Schulnote von 1 oder 2 bewertet (Tab. 11).

\begin{tabular}{|l|c|c|c|c|c|c|c|c|c|}
\hline \multirow{2}{*}{ Item } & \multicolumn{3}{|c|}{$\begin{array}{l}\text { "Ich bewerte die } \\
\text { Kasuistik mit..." }\end{array}$} & \multicolumn{2}{c|}{$\begin{array}{c}\text { "Ich habe viel lernen } \\
\text { können in der } \\
\text { Kasuistik.“ }\end{array}$} & \multicolumn{2}{|c|}{$\begin{array}{c}\text { "Ich konnte dem } \\
\text { Fallbeispiel gut } \\
\text { folgen.“ }\end{array}$} \\
\cline { 2 - 10 } & PAL & VPL & p-Wert & PAL & VPL & p-Wert & PAL & VPL & p-Wert \\
\hline Bauchschmerz & 1 & 2 & 0.0837 & 2 & 2 & 0.5350 & 1 & 2 & 0.3128 \\
\hline Hypertonie & 2 & 2 & $>0.9999$ & 2 & 2 & 0.2604 & 1 & 1 & 0.8953 \\
\hline Husten & 1 & 1 & $>0.9999$ & 2 & 1 & 0.1845 & 1 & 1 & 0.5497 \\
\hline Kreuzschmerz & 1.5 & 2 & 0.8987 & 1.5 & 2 & 0.5084 & 1 & 1 & 0.1818 \\
\hline Dyspnoe & 2 & 2 & 0.7146 & 2 & 2 & 0.3566 & 1 & 1 & 0.2411 \\
\hline Kopfschmerz & 1 & 2 & 0.1408 & 1 & 1 & $>0.9999$ & 1 & 1 & 0.6308 \\
\hline Schwangerschaft & 2 & 2 & 0.1666 & 2 & 1.5 & 0.0254 & 1 & 2 & 0.0076 \\
\hline Diabetes & 1 & 2 & 0.2353 & 2 & 2 & 0.3955 & 1 & 1 & 0.7610 \\
\hline
\end{tabular}

Tabelle 11a: Seminarevaluationen der PAL Gruppe und VPL Gruppe (Mediane und pWerte) 


\begin{tabular}{|l|c|c|c|c|c|c|}
\hline \multirow{2}{*}{ Item } & \multicolumn{3}{|c|}{$\begin{array}{c}\text { "Ich weiß nicht viel } \\
\text { mehr als vorher." }\end{array}$} & \multicolumn{3}{|c|}{$\begin{array}{c}\text { "Die Menge an } \\
\text { Lehrinhalt war } \\
\text { angemessen." }\end{array}$} \\
\cline { 2 - 7 } & PAL & VPL & p-Wert & PAL & VPL & p-Wert \\
\hline Bauchschmerz & 6 & 5 & 0.1430 & 2 & 1 & 0.6374 \\
\hline Hypertonie & 5.5 & 5 & 0.0876 & 1.5 & 1.5 & $>0.9999$ \\
\hline Husten & 6 & 5 & 0.0963 & 2 & 1 & 0.5174 \\
\hline Kreuzschmerz & 6 & 5 & 0.0080 & 1 & 1 & 0.8428 \\
\hline Dyspnoe & 6 & 4 & 0.0059 & 2 & 1.5 & 0.5222 \\
\hline Kopfschmerz & 6 & 5.5 & 0.1100 & 1 & 1 & 0.5735 \\
\hline Schwangerschaft & 5 & 5 & 0.5983 & 1 & 1 & $>0.9999$ \\
\hline Diabetes & 6 & 4 & 0.0038 & 1 & 1 & 0.3461 \\
\hline
\end{tabular}

Tabelle 11b: Seminarevaluationen der PAL Gruppe und VPL Gruppe (Mediane und pWerte) 


\section{DISKUSSION}

Ziel der vorliegenden Studie war es, PAL und VPL erstmals im Hinblick auf den Erwerb von allgemeinmedizinischen Basiskompetenzen innerhalb einer studentisch betriebenen Poliklinik zu vergleichen. Insgesamt zeigen die Ergebnisse der vorliegenden Arbeit keinen signifikanten Unterschied zwischen den zwei Unterrichtsformaten im Hinblick auf einen langfristigen theoretischen Wissenszuwachs und den Erwerb praktischer Fertigkeiten. Die Qualität, der Inhalt und die Verständlichkeit der PAL Seminare und VPL Seminare wurden von den Studenten ebenfalls ähnlich bewertet. Ein signifikanter Unterschied zugunsten der VPL Gruppe ergab sich lediglich im kurzfristigen theoretischen Wissenszuwachs.

In der Einschätzung ihrer allgemeinmedizinischen Grundkenntnisse und Fähigkeiten zeigten sich die Studenten der PAL Gruppe deutlich selbstsicherer als die der VPL Gruppe und der KG.

\subsection{Theoretische Tests}

\subsubsection{Theoretischer Langzeit-Test}

Im theoretischen Vortest erzielten alle drei Gruppen sehr ähnliche Ergebnisse, sodass davon ausgegangen werden kann, dass sie ein gleichwertiges Wissensniveau zu Beginn der Studie besaßen und so das Vergleichen der Gruppen für den Nachtest möglich ist.

Im theoretischen Nachtest zeigten sowohl die PAL Gruppe als auch die VPL Gruppe insgesamt einen Wissenszuwachs von jeweils fast $40 \%$ und schnitten somit gleich gut ab. Diese Ergebnisse deuten darauf hin, dass der Einsatz von virtuellen Patienten für die Vermittlung allgemeinmedizinischen Grundwissens ebenso effektiv ist wie das häufig in Student-run Free Clinics eingesetzte PAL Format.

Auch die KG zeigte einen signifikanten Wissenszuwachs im theoretischen Nachtest, welcher jedoch nur etwa 10\% betrug. Der Wissenszuwachs der KG ist vermutlich auf den curricularen Unterricht zurückzuführen, welcher unter anderem einführende Veranstaltungen in der Inneren Medizin, Chirurgie, Pharmakologie und Mikrobiologie beinhaltete und somit ähnliche 
Krankheitsbilder wie im Wahlfach der Studentischen Poliklinik thematisierte. ${ }^{98}$ Insbesondere die Leitsymptome und dazugehörigen Kasuistik-Themen Bauchschmerz (Gastritis), Anhaltender Husten (Tuberkulose), arterielle Hypertonie, Dyspnoe und Leistungsminderung (Anämie) und Polyurie (Diabetes) waren Gegenstand des curricularen Unterrichts. Die Themenkomplexe Schwangerschaft, Kopfschmerz und Kreuzschmerz wurden nicht unterrichtet. Dies lässt sich in den theoretischen Langzeit-Ergebnissen der KG wiedererkennen. So zeigte sich kein Wissenszuwachs zum Thema Schwangerschaft und in den Themenbereichen Kreuzschmerz und Kopfschmerz verschlechterte sich die KG sogar.

Sowohl die PAL Gruppe als auch die VPL Gruppe hingegen zeigten in den drei genannten Themen einen signifikanten Wissenszuwachs. So lässt sich möglicherweise erklären, dass die KG durch den curricularen Unterricht einen Wissenszuwachs in denjenigen Themenbereichen erzielen konnte, die auch im Wahlfach der Studentischen Poliklinik relevant waren.

Warum aber die beiden Testgruppen insgesamt besser abschnitten als die KG, lässt sich auf unterschiedliche Weise begründen. Sowohl durch das PAL als auch durch das VPL Format wird die aktive Rolle der Studenten gefördert.41,43,70,71

Eine aktive Rolle im Lernprozess zu übernehmen bedeutet, dass die Studenten selbstständig Entscheidungen treffen und auf ihr zuvor angeeignetes Wissen zurückgreifen oder sich neues Wissen aneignen müssen. Sie sind dadurch Situationen exponiert, in denen sie auf Fragen des Tutors oder des e-Learning Programms antworten müssen. Der Lernprozess wird durch die Studenten mitgesteuert und rückt den Lernenden in den Mittelpunkt. Zum einen ist dies im PAL Format zu beobachten. Hierzu schreiben Boud et $\mathrm{al}^{31(\mathrm{p} 416)}$, dass die Studenten im PAL ihre eigenen Lernbedürfnisse definieren und lernen wie man lernt.

Andererseits trifft dies auch auf das VPL Format zu. Das e-Learning Programm Lernbar mit integrierten virtuellen Patienten erfordert, ähnlich wie die von Bryce et $\mathrm{al}^{70(\mathrm{p} 9)}$ beschriebenen e-Learning Kurse, einen aktiven Umgang mit Informationen und deren Zusammenführung: „It provides a relevant context for learning, is problem-based, requires the user to actively handle and synthesise information, [...]“. Das aktive Einbeziehen der Studenten und die direkte Kommunikation und Interaktion mit ihnen sind Faktoren, die für effektives Lernen 
vordergründig sind. So schreiben auch Sutkin et al ${ }^{41(p 457)}$ in ihrer Studie zur Frage, was einen guten klinischen Lehrer in der Medizin ausmacht:

„This study suggests that excellent teaching, although multifactorial, transcends ordinary teaching and is characterized by inspiring, supporting, actively involving and communication with students. These activities produce an emotional arousal in the students."

In den Kasuistiken der PAL Gruppe und der VPL Gruppe wurde die Autonomie der Studenten und auch das selbstgesteuerte Lernen gefördert. Diese zwei Elemente scheinen auch laut Irby et al $^{104}$ Kernpunkte für ein effektives Lernen zu sein.

Bryce et $\mathrm{al}^{70(\text { p20) }}$ beschrieben zusätzlich, dass mit den virtuellen Patientenfällen die Studenten die Krankheitsbilder ganzheitlich kennenlernen können: „It could be argued that because students can work through a complete patient problem, including test ordering, to reach a diagnosis, they are able to build a more integrated clinical picture of an illness." Dies war auch in den Kasuistiken der PAL Gruppe und VPL Gruppe der Fall, da vom Leitsymptom, über Differentialdiagnosen bis $\mathrm{zu}$ der korrekten Therapie die einzelnen Krankheitsbilder in ihrer Gesamtschau erarbeitet wurden. Im curricularen Unterricht an echten Patienten kann dies nicht immer realisiert werden. Nur selten betreuen die Studenten Patienten von Anfang bis Ende.

Wie diese Quellen, deuten auch unsere Ergebnisse darauf hin, dass die beiden Lehrformate mit hoher Qualität das eigenständige Auseinandersetzen mit der jeweiligen Thematik in einer Lerner-zentrierten Form fördern und so ein Wissenszuwachs über einen längeren Zeitraum erreicht werden kann. Besonders hervorzuheben ist, dass die Studenten sich durch das PAL Format und VPL Format bewusster mit den Lerninhalten auseinandersetzen, da die aktive Einbeziehung ihres Wissens und Handelns herausfordernd wirkte. Die integrative Teilnahme der Studenten am Lehrprozess kann dazu geführt haben, dass sie motiviert waren mehr zu leisten und kann einen möglichen Grund für den Wissenszuwachs darstellen.

Ein weiterer motivierender Faktor kann das Wissen darüber sein, dass die Studenten sich nach Teilnahme am Seminar in der Sprechstundenarbeit der Studentischen Poliklinik engagieren und dort echten Patientenkontakt haben 
würden. Die theoretische Vorbereitung im PAL Seminar und im VPL Seminar wurde daher wahrscheinlich sehr ernst genommen.

Hinzu kommt, dass die präsentierten Fallbeispiele realistisch gestaltet waren und den Studenten als Vorschau auf die Sprechstundenarbeit dienten. Schutte et $\mathrm{al}^{23(\text { pp.3-6) }}$ beschrieben in diesem Zusammenhang die drei Eigenschaften Verantwortung, Authentizität und Zusammenarbeit, die im Rahmen von Studentrun Free Clinics lernmotivierend wirken. Diese Erkenntnis von Schutte et al ${ }^{23(p 7)}$ unterstützt die Annahme, dass die authentischen Fallbeispiele in den PAL Seminaren und VPL Seminaren lernfördernd wirkten.

Da der Nachtest als benotete Klausur im Wahlfach der Studentischen Poliklinik verwendet wurde, kann davon ausgegangen werden, dass die Studenten eine gute Leistung erbringen wollten. Dies könnte ebenfalls einen Einfluss auf die Ergebnisse der theoretischen Langzeitprüfung gehabt haben. Über die Art einer Bewertung und der daraus resultierenden Leistung wurde in früheren Studien ein klarer Zusammenhang gefunden. Raupach et al $^{105(\mathrm{p} 2)}$ zufolge können formative Bewertungen (Feedback) einen geringeren Lernanreiz erzeugen als summative (benotende Bewertungen). Insbesondere gilt dies für Bewertungen, welche wie im Falle des theoretischen Nachtests, am Ende des Kurses den Lernerfolg zusammenfassen und unabhängig vom zuvor angewandten Lehrformat tendenziell eine Leistungssteigerung der Studenten aufweisen. ${ }^{105(p 9)}$

\subsubsection{Theoretischer Kurzzeit-Test}

In der Gesamtschau erzielte die VPL Gruppe in den theoretischen Kurzeit-Tests ein signifikant besseres Ergebnis als die PAL Gruppe. Dieser signifikante Unterschied kann mehrere Ursachen haben.

In den Seminaren der VPL Gruppe konnten sich die Studenten innerhalb der Kasuistik frei im Patientenfall bewegen und so vergessene Informationen erneut aufrufen. Zudem wurden elektronische Verweise zu weiterführender Literatur bereitgestellt. Darüber hinaus ist zu berücksichtigen, dass sich die PAL-Seminare in der Regel über maximal zwei Stunden erstreckten, während die VPL Seminare keine zeitliche Begrenzung hatten. In diesem Zusammenhang ist die zeitliche Flexibilität als Vorteil von e-Learning mit virtuellen Patienten hervorzuheben. Auch Fleetwood et $\mathrm{al}^{67(\mathrm{p} 102)}$ betonen dies im Rahmen ihrer Studie zu einem 
computer-unterstützten medizinischen Lernprogramm mit den Worten „It allows students to self-pace their learning, soliciting expert information as needed and spending as little or as much time on any given case as the learner believes appropriate."

Auch Krüger-Brand ${ }^{68}$ schrieb im Ärzteblatt: „Den Studenten ermöglichen sie [computergestützte Lernsysteme, Anm. d. Verf.] einen zeit-und ortsunabhängigen Zugang zu Lerninhalten und ein selbstbestimmtes interaktives Aneignen des Lernstoffes im Selbststudium“. Individuelle Lerngeschwindigkeiten und Lernvoraussetzungen werden somit berücksichtigt, ${ }^{67}$ und könnten die besseren Ergebnisse der VPL Gruppe erklären.

Unterstützt wird diese These auch von Tworek et $\mathrm{al}^{69(\mathrm{p} 156)}$, die zeigen konnten, dass virtuelle Patienten den Fokus der Inhalte in eine Lerner-zentrierte Perspektive fördern. Wie diese Quellen, lässt sich auch aus unseren Ergebnissen schlussfolgern, dass keiner der Studenten während der VPL Seminare übergangen wurde, da niemand über- oder unterfordert war. Jeder Student konnte eigenständig sein Lerntempo und sein Lernpensum bestimmen.

Die Lerner-zentrierte Perspektive wird auch weiter von Fleetwood et al ${ }^{67}$ betont. So heißt es zum einen: „Computer-based Learning can adapt to students“ crowded schedules, enabling students to access the program at their convenience from diverse locations (including rotation sites and their homes)“.67(p102) Die Studenten der VPL Gruppe konnten ebenfalls individuell bestimmen, wann sie den jeweiligen Fall bearbeiten wollten. Möglicherweise waren sie zu den selbstgewählten Bearbeitungszeitpunkten konzentrierter.

Zum anderen schreiben sie zu den Vorteilen von computer-unterstütztem Lernen: „It overcomes the problem of variability in small-group quality, as when a smallgroup facilitator is unfocused or gives misinformation, or when group participants have interpersonal issues that impede learning. ${ }^{67(p 102)}$ Der Lernprozess ist somit individuell vom Lernenden abhängig, welcher im Mittelpunkt des Geschehens steht und nicht von Tutor und Peer-Gruppe, die den Lernfortschritt auch negativ beeinflussen könnten.

Durch Berücksichtigung der individuellen Lernvoraussetzungen in der VPL Gruppe wurde zudem die Selbstständigkeit der Studenten gefördert. Dies wiederum unterstützt die aktive Auseinandersetzung mit den Themen und eigenen Interessen nachzugehen. 
Das bessere Abschneiden der VPL Gruppe in den theoretischen Kurzzeit-Tests könnte zudem durch den in der Literatur erwähnten testing effect erklärt werden. ${ }^{106,107(p 181)}$ Dieser Effekt beschreibt, dass Prüfungen neben der Bewertung von Studenten auch lernfördernd wirken können. ${ }^{106}$ Dabei wird unterschieden zwischen der direkten und indirekten Auswirkung von Prüfungen. ${ }^{106,107(p p 181-182)}$ Larsen, Butler und Roediger ${ }^{106(p p 959-960)}$ erklären den direkten Effekt dadurch, dass Studenten sich besser an gelehrte Themen erinnern, wenn sie wissen, dass hierzu ein Test stattfindet. Der indirekte Effekt beschreibt, dass das häufigere Durchführen von Tests die Lernzeit von Studenten erhöht und bessere Lernstrategien entwickelt werden. ${ }^{106(p 960)}$ Möglicherweise ist dieser testing effect aber teilweise auch auf die PAL Gruppe anwendbar, da den Teilnehmern dieser Gruppe auch bewusst war, dass am Ende eines jeden Kasuistikseminars ein Kurzzeit-Test erfolgen würde. Der Unterschied ist jedoch, dass während des Seminars keine schriftlichen Zwischenfragen gestellt wurden und die Dauer des Seminars begrenzt war. Die VPL Gruppe hingegen hatte die Möglichkeit sich intensiver auf die Tests vorzubereiten, da keine zeitliche Begrenzung gegeben war. Außerdem enthielten die Kasuistikseminare der VPL Gruppe zwischenzeitliche Single -und MultipleChoice-Fragen. Teilweise ähnelten diese Fragen denen am Ende des Seminars, sodass auch hier ein Zusammenhang möglich ist.

Ein weiterer Aspekt, den man bedenken sollte, wird von Kerres et al ${ }^{108(p 1)}$ als sogenannter Neuigkeitseffekt beschrieben: „Tatsächlich können neue Medien zu einer Steigerung der Motivation bei Lernenden beitragen. Diese Beobachtung beruht insbesondere auf dem sogenannten „Neuigkeitseffekt“ [...]“.108(p1) Durch ein ansprechend gestaltetes Design und ein didaktisch gut aufbereitetes neues Lernformat, kann es sein, dass die Studenten der VPL Gruppe etwas motivierter waren, die Fälle zu bearbeiten als die Studenten der PAL Gruppe. Jedoch scheint der Effekt „[...] eher von kurzer Dauer [...]“ zu sein, ${ }^{108(p 1)}$ und kann daher nicht vollständig die besseren Ergebnisse der VPL Gruppe erklären. Vielmehr sind die zuvor genannten Elemente des e-Learnings, die die individuellen Voraussetzungen des Lernenden berücksichtigen, der wahrscheinlichere Grund für den besseren kurzzeitigen Wissenszuwachs. 


\subsection{Praktische Tests}

\subsubsection{Wahlfach OSCE}

Die Ergebnisse im Wahlfach OSCE zeigten einen ähnlichen praktischen Kompetenzerwerb der PAL Gruppe und der VPL Gruppe. Dass das Erlernen praktischer Fertigkeiten mittels Peer-assisted Learning effektiv ist, konnte bereits mit vorherigen Studien gezeigt werden und wurde auch von Seifert et $\mathrm{al}^{2}$ im Rahmen des Wahlfachs der Studentischen Poliklinik Frankfurt untersucht. Hierzu schrieben $\mathrm{sie}^{2(\mathrm{p} 521)}$ :

"These studies support our assumption that the use of trained peer teachers, in addition to curricular teaching by physicians, may result in a large increase in theoretical knowledge and improve clinical skills. The results of our study quantify this increase for the first time in a curricular setting and in the context of an SRFC [Student-run Free Clinics, Anm. d. Verf.] project."

In der vorliegenden Arbeit konnte zusätzlich gezeigt werden, dass das VPL Format für das Erlernen klinisch praktischer Fertigkeiten, der körperlichen Untersuchung und Anamneseerhebung ebenso effektiv ist. Man kann annehmen, dass die in den VPL Seminaren enthaltenen Videos und Bilder die Peer-Tutoren der PAL Gruppe ersetzen könnten. In der Literatur ist die Meinung bezüglich der Effektivität des Erlernens von praktischen Fertigkeiten anhand von Videos gespalten. Karimi Mouneghi et al ${ }^{109(\mathrm{p} 29)}$ beispielsweise konnten in ihrer Studie folgendes resümieren:

"Results show that training through video is not as effective as demonstration. The students' grades, however, were high enough in both ways. Video-based educational method can be a suitable substitution when we are not able to perform the demonstrating method or when it is not cost-effective."

Auch Devi, Khandelwal und Das ${ }^{110}$ meinen, dass es keinen Ersatz für die direkte klinische Demonstration gibt, aber sehen den video-unterstützten Unterricht als mögliche Ergänzung zu der traditionellen Lehre am Krankenbett.

Einige Studien konnten jedoch belegen, dass das Lernen mit Videos als Grundlage effektiv in der medizinischen Ausbildung sein kann. ${ }^{111,112(p 5-6)}$ „Audiovisual material provides a rich medium for teaching and learning. Video 
can effectively communicate complex information to a student and, if used creatively, can become a powerful expressive tool." wird von Tayade et al ${ }^{111(p 16)}$ argumentiert. Sie führten eine Studie durch, in der Studenten im Fach Radiologie zu wichtigen thorakalen Notfällen mittels Videos geschult wurden und fanden heraus, dass das Video-unterstütze Lernen eine effektive Lehrmethode ist. ${ }^{111(p 17)}$ Die Studenten waren der Meinung, dass Video-unterstütztes Lernen den Merkprozess der unterrichteten Inhalte und das Abrufen von neuem Wissen unterstützt und vor allem den Lernprozess fördert, da es eine abwechslungsreiche Lehrmethode darstellt. ${ }^{111(p 15)}$

Schwerdtfeger et al ${ }^{112(\mathrm{p} 2)}$ untersuchten die Wirksamkeit eines video-unterstützten 4-Stufen-Ansatzes. Der sogenannte 4-Stufen-Ansatz wird zur Vermittlung von klinisch-praktischen Fertigkeiten in Kursen zu Reanimation und Trauma eingesetzt. ${ }^{112(\mathrm{p} 2)}$ Eine Kontrollgruppe absolvierte den Kurs im traditionellen Format, in welchem die klinischen Fertigkeiten während der ersten beiden Schritte von einem Ausbilder vorgezeigt wurden. In der Studiengruppe wurde der Ausbilder jedoch durch Videos ersetzt. ${ }^{112(p p 2-3)}$ Anschließend absolvierten beide Gruppen einen OSCE, in welchem beide Gruppen ähnlich gute Ergebnisse ohne signifikante Unterschiede erzielten. ${ }^{112(\mathrm{pp} 3-4)}$ Darüber hinaus bewertete die Studiengruppe den relativen Wissenszuwachs nach dem Kurs höher als die Kontrollgruppe. ${ }^{112(\mathrm{p} 3)}$ Wie diese Studien lassen auch unsere Ergebnisse annehmen, dass das Erlernen von praktischen Fertigkeiten und insbesondere der körperlichen Untersuchung mit Videomaterial in den VPL Seminaren genauso effektiv ist wie im PAL Seminar mithilfe von Peer-Tutoren. An dieser Stelle ist außerdem der Gedanke aufzugreifen, dass sowohl die PAL Gruppe als auch die VPL Gruppe im Modul 1 des Wahlfachs (Tab. 2) das gleiche Training erhielten, was ebenfalls einen Grund für das ähnlich gute Abschneiden beider Gruppen darstellen könnte.

Eine Fragestellung, die noch weiterer Untersuchungen bedarf, ist jedoch, ob den Studenten Feedback fehlte und wie sicher sie sich - unabhängig von den hier vorliegenden objektiven Ergebnissen - fühlten. Dies wird im Abschnitt zur Selbsteinschätzung weiter diskutiert. 


\subsubsection{Curricularer OSCE}

Im curricularen OSCE schnitten sowohl die PAL Gruppe und VPL Gruppe als auch die KG ähnlich gut ab. Es zeigte sich somit kein signifikanter Unterschied zwischen den drei Gruppen. Bei der OSCE-Station „Untersuchung Kardiologie, Angiologie oder Pulmologie" erzielte die KG sogar ein signifikant besseres Ergebnis. So stellt sich die Frage, warum die KG genauso gut oder teilweise besser war als die Testgruppen, die ein zusätzliches praktisches Training absolviert hatten. Die Bedeutung einer Prüfung, insbesondere in der medizinischen Ausbildung, beeinflusst das Lernen und das Lernumfeld von Studenten. ${ }^{113(\mathrm{p} 7)}$ In der Literatur wird das Phänomen Assessment drives Learning ${ }^{14,115(\text { p202) }}$ beschrieben, welches aus dem Englischen interpretiert bedeutet, dass Bewertungen das Lernen vorantreiben. Im engeren Sinne können wir aus den hier präsentierten Ergebnissen davon ausgehen, dass die notengebende Abschluss-Prüfung in Form eines curricularen OSCEs die Studenten dazu motiviert hat, intensiver für die Prüfung zu lernen. So schreiben beispielsweise auch Wormald et al. ${ }^{115(\mathrm{p} 202)}$ in ihrer Arbeit zur Untersuchung über Motivationsgründe ein Fach zu erlernen "The results of this survey indicate a significant link between the weighting of a subject within an assessment scheme and medical student's reported motivation towards learning the subject."

Kromann, Jensen und Ringsted führten eine Studie zur Auswirkung einer finalen Prüfung nach absolviertem Reanimationskurs durch und schreiben hierzu116(p24): "This study suggests that testing as a final activity in a resuscitation skills course increases learning outcome compared with an equal amount of time spent in practice. [...] Accordingly, our study on skills learning [...] corresponds on the testing effect and indicates that the testing effect can be reproduced in skills learning."

Demzufolge ist es möglich, dass auch der curriculare OSCE in dieser Arbeit die Studenten aller drei Gruppen beeinflusst hat und ähnliche leistungsstarke Ergebnisse resultierten.

Weiterhin muss auch erwähnt werden, dass die Stichprobengröße der KG mit nur elf Teilnehmern sehr gering und somit eventuell nicht repräsentativ genug war. Offen bleibt die Frage, ob die Gender-Verteilung einen Einfluss auf die Ergebnisse haben könnte und die teilweise besseren Ergebnisse der KG erklären 
könnte. Die KG hatte ausschließlich weibliche Teilnehmer während die PAL Gruppe einen männlichen Anteil von 15\% und die VPL Gruppe von 30\% hatte. In einigen Studien wurde bereits gezeigt, dass Frauen in klinisch-praktischen Fertigkeiten besser abschneiden als Männer. ${ }^{117-120}$ Ferguson et al ${ }^{118}$ konnten in einer systematischen Literaturdurchsicht zeigen, dass Frauen in klinischen Prüfungen während der medizinischen Ausbildung besser abschnitten als Männer. „Our study shows women perform better than men across a variety of skills and disciplines [...]" schlussfolgerten beispielsweise auch Haist et al ${ }^{117(p 196)}$. Dies könnte somit möglicherweise erklären, dass die KG mindestens genau so gute Ergebnisse im curricularen OSCE erzielte wie die beiden Testgruppen obwohl sie kein zusätzliches Training erhielten. Dennoch sollte dies eher hintergründig betrachtet werden, da die Stichprobengröße der Gruppen relativ klein war.

\subsection{Selbsteinschätzung}

Die Teilnahme am Wahlfach der Studentischen Poliklinik führte zu einer besseren Selbsteinschätzung der Studenten bezüglich ihrer allgemeinmedizinischen Grundkompetenzen als die alleinige Teilnahme am curricularen Unterricht. Sowohl die PAL Gruppe als auch die VPL Gruppe erhielten durch die Wahlfachteilnahme zusätzliches und qualitätsreiches Training. Ferner engagierten die Studenten sich ehrenamtlich in der Sprechstunde der Studentischen Poliklinik und übernahmen hier Verantwortung, was ihr Selbstwertgefühl und, damit assoziiert, ihre Sicherheit in den befragten Kompetenzen erklären kann. Die Teilnahme an einer Student-run Free Clinic, wie der Studentischen Poliklinik Frankfurt, fördert Stephens et $\mathrm{al}^{12}$ zufolge die Autonomie der Studenten. Williams et $\mathrm{al}^{24(\mathrm{p} 306)}$ betonen die Wichtigkeit der Autonomie in der medizinischen Ausbildung und argumentieren:

"These findings highlight the importance of experimenting with the promotion of autonomy-supportive rather than controlling climates in medical schools as a way of facilitating students' autonomous motivation and, in turn, improving their learning, performance, and psychological wellbeing." 
Auch im Wahlfach und insbesondere während der Sprechstundenarbeit der Studentischen Poliklinik wurde die Autonomie der Studenten gefördert und könnte unter anderem die gute Selbsteinschätzung beider Wahlfachgruppen erklären. Die frühe praktische Erfahrung in einem behüteten Rahmen, die der KG fehlte, bekräftigt zusätzlich die subjektiv gewonnene Kompetenz der Studenten der PAL und VPL Gruppe.

Es bleibt offen, warum die PAL Gruppe sich in sechs befragten Items signifikant besser einschätze als die VPL Gruppe. Ein möglicher Grund hierfür ist das Lehrformat. In der PAL Gruppe wurden die Teilnehmer durch Peer-Tutoren geschult. Diese fungierten als Vorbilder und eine Identifikation mit innen liegt sehr nahe. ${ }^{41(p 457)}$ Vor allem Tutoren, die das Wahlfach bereits absolviert hatten, können motivierend wirken. ${ }^{42\left(\mathrm{p}^{221}\right)}$ Die bereits erwähnte und von Bugaj et al ${ }^{38}$ beschriebene kognitive Kongruenz scheint die bessere Selbsteinschätzung der Studenten in der PAL Gruppe ebenfalls zu unterstützen und den wesentlichen Unterschied zur VPL Gruppe auszumachen. Die Teilnehmer der VPL Gruppe wurden ähnlich der PAL Gruppe aktiv in die Kasuistikseminare einbezogen, was somit ein intensiveres Auseinandersetzen mit der Thematik bedeutete und ein effektives Lernen förderte. ${ }^{70,72}$ Zusätzlich wurde versucht in den VPL Seminaren durch Feedback während der Fälle die Tutor-Komponente zu ersetzen. Trotzdem kann der Grund für die unterschiedliche Selbsteinschätzung fehlendes mündliches Feedback durch einen Peer-Tutor über die Leistung der Studenten sein. Verschiedene Studien hoben die Bedeutung von strukturiertem Feedback in der klinischen Ausbildung hervor. 54,58,59,121,122 Tworek et al ${ }^{69(p p 155-156)}$ warfen in einer Studie auf, dass das Lernen mit virtuellen Patienten womöglich kein effektives Feedback geben könne. In der vorliegenden Arbeit wurde versucht so viel Feedback während der Bearbeitung eines VPL Seminars zu geben wie möglich. Diese Form von Feedback orientierte sich am adaptiven Feedback, wie von Tworek et al ${ }^{69(p 157)}$ vorgeschlagen wurde. Hierbei kommen mehrere Feedback-Ebenen, wie beispielsweise die schrittweise Aufdeckung der richtigen Diagnose oder die Bereitstellung von Expertenmeinungen, vor. ${ }^{69(p 157)}$

Trotzdem fehlte in den VPL Seminaren die mündliche und persönliche Komponente von Peer-Tutoren, sodass zum einen die Identifikationsmöglichkeit mit älteren Studenten wegfiel und andererseits kann angenommen werden, dass 
das virtuelle Feedback den Studenten nicht so nahe ging wie Feedback von „Angesicht zu Angesicht“.

Diese Überlegung zur Klärung der vorliegenden Ergebnisse dieser Arbeit wird auch von Williams et $\mathrm{al}^{24(\mathrm{p} 304)}$ weiter unterstützt. So meinen $\mathrm{sie}^{24(\mathrm{p} 304) \text { : }}$

„Autonomy-supportive instructors may hold high standards, set limits, make recommendations, and give honest feedback, just as controlling instructors do these things in an understanding, encouraging, nonjudgmental style rather than a harsh, demanding, critical style."

So wird zum einen noch einmal die Wichtigkeit der Autonomie in der medizinischen Ausbildung hervorgehoben. Zum anderen meinen Williams et $\mathrm{al}^{24(\mathrm{p} 304)}$, dass das Feedback-Geben in einer Autonomie-fördernden Art movierend für Studenten sein kann. Diese Form von Feedback wurde auch in der PAL Gruppe angeboten und kann somit möglicherweise die bessere Selbsteinschätzung widerspiegeln.

Zusammenfassend ist die Teilnahme am Wahlfach der Studentischen Poliklinik und dabei vor allem die aktive Beteiligung in der Sprechstundenarbeit fördernd für eine verbesserte Selbsteinschätzung. Die unterschiedlichen Lehrformate differenzieren anschließend zusätzlich die Auswirkungen auf die Selbsteinschätzung. So spielt die kognitive Kongruenz der PAL Gruppe mit direktem mündlichem Feedback der Peer-Tutoren die wahrscheinlich entscheidende Rolle und kann zu einer höheren Selbsteinschätzung führen.

Objektiv betrachtet gibt es zwar keinen signifikanten Unterschied im Bereich des langzeitigen Wissen-und Kompetenzerwerbs zwischen der PAL Gruppe und der VPL Gruppe, trotzdem muss das VPL Format hinsichtlich individuelleren Feedbacks in Zukunft verbessert werden.

\subsection{Seminarevaluation}

Die ausgewerteten Evaluationen zu den Kasuistikseminaren zeigen, dass die PAL Gruppe und VPL Gruppe hinsichtlich Verständlichkeit des Fallbeispiels, Menge an Lehrinhalten und neugewonnenem Wissen gleich zufrieden waren. Anhand dieser Ergebnisse lässt sich schlussfolgern, dass bei guter Aufbereitung von virtuellen Patientenfällen die Peer-Tutoren ersetzt werden können. 
Außerdem zeigt sich, dass durch den schrittweisen Aufbau der Seminare in den VPL Gruppen die Inhalte verständlich und ähnlich der PAL Seminare waren. So konnte anhand vieler Hilfsfunktionen, wie beispielsweise Expertenmeinungen oder Erläuterungen von Falschantworten zu den Zwischenfragen eine Art roter Faden erkannt werden, der die Teilnehmer durch die Thematik führte, so wie es die Peer-Tutoren zur Aufgabe hatten. Wie virtuelle Patientenfälle am besten aufbereitet werden, wurde auch von der American Association for Medical Colleges $^{51(p 12)}$ auf die Prinzipien von Gagné und Piaget ${ }^{123}$ angewandt. Einige dieser Vorschläge wurden in den VPL Seminaren des Wahlfachs ebenfalls umgesetzt. So wird unter anderem vorgeschlagen, dass in den Fallseminaren Multiple-Choice Fragen präsentiert und Feedback angeboten werden sollte. ${ }^{51(\mathrm{p} 13)}$ Zudem sollte zu Beginn einer Kasuistik ein Aufmerksamkeit erregendes Medium wie beispielsweise ein Bild eingesetzt werden und klare Lernziele definiert werden, was ebenfalls in den VPL Fällen umgesetzt wurde. ${ }^{51(\mathrm{p} 13)}$ Ferner wurde die Idee, dass am Ende einer Lerneinheit die Studenten anhand von MultipleChoice Fragen zum zuvor erlernten Material „geprüft“ werden sollten, in dieser Arbeit umgesetzt. ${ }^{51(\mathrm{p} 13)}$

Insgesamt scheint es, dass die virtuellen Patientenfälle im Wahlfach der Studentischen Poliklinik gut aufbereitet und von den Studenten angenommen wurden.

\subsection{Schlussfolgerung und Ausblick}

Die vorliegende Arbeit vergleicht erstmals den Einsatz von virtuellen Patienten mit der traditionelleren Lehrmethode Peer-assisted Learning im Rahmen einer Student-run Free Clinic.

Die Ergebnisse dieser Studie bestätigen die Hypothese, dass Seminare im VPL Format genauso effektiv sind wie Seminare im PAL Format und zeigen, dass beide Unterrichtsformen in Student-run Free Clinics zur Vermittlung allgemeinmedizinischer Basiskompetenzen genutzt werden können.

Da es nur wenige kontrolliert randomisierte Studien zu VPL und der medizindidaktischen Wirksamkeit gibt, ist zu erwähnen, dass diese Arbeit einen Anstieg des kurz-und langfristigen Wissens zeigen konnte. Darüber hinaus können grundlegende praktische Fertigkeiten wie Anamnese-Erhebung und die 
körperliche Untersuchung anhand von web-basierten Fällen mit virtuellen Patienten vermittelt werden.

Die VPL Seminare wurden von den Studenten gut angenommen und sind seither fester Bestandteil des Wahlfachs der Studentischen Poliklinik Frankfurt. Seit dem Wintersemester 2017/2018 werden die Studenten jedoch gemischt im PAL und VPL Format unterrichtet. Zwei Gruppen mit je 20 Teilnehmern erhalten jeweils vier Seminare im PAL Format und weitere vier im VPL Format. So wird zum einen gewährleistet, dass alle Studenten die gleiche Ausbildung erhalten und zum anderen ein vielseitiger Lehransatz genutzt, der ein effektives Lernumfeld fördert. ${ }^{124}$ Hinzu kommt, dass die neue Generation von Studenten blended learning, also eine Kombination von traditionellen Lehrmethoden und e-Learning mittlerweile als selbstverständlich sieht. ${ }^{65(\mathrm{p} 2)}$ Die Studenten haben also die Erwartung verschiedene Medien für den Kompetenzerwerb zu nutzen. Virtuelle Patienten bieten hier in Kombination mit dem Peer-assisted Learning vielfältige Möglichkeiten.

Durch die Kombination von PAL und VPL können pro Jahr 40 Studenten am Wahlfach der Studentischen Poliklinik teilnehmen, fast doppelt so viele wie zu Anfängen des Wahlfachs. ${ }^{2(p 515)}$ Trotzdem ist die gleiche Anzahl an Tutoren tätig, sodass sich eine gewisse Kosteneffizienz zeigt. Natürlich ist, wie im einleitenden Abschnitt der Arbeit erklärt wurde, anfänglich der inhaltliche Aufwand für das Erstellen von virtuellen Patientenfällen hoch. ${ }^{51(p 7)}$ Dies zeigte sich auch im Rahmen des Wahlfachs der Studentischen Poliklinik. Zu Beginn mussten die Fälle entwickelt und zugänglich gemacht werden. Erst mit den folgenden Jahrgängen ist ein geringerer Aufwand zu erkennen gewesen und spricht somit für den Einsatz von virtuellen Patienten.

Die Unabhängigkeit in Ort und Zeit und die Lerner-zentrierte Lehre sind in Kombination mit den Ergebnissen dieser Arbeit Faktoren, die stark für den häufigeren Einsatz von virtuellen Patienten in der Vermittlung von allgemeinmedizinischem Grundwissen und Fertigkeiten im Rahmen von Studentrun Free Clinics sprechen. In Zukunft müssen die virtuellen Patientenfälle jedoch individuelleres Feedback enthalten und müssen kontinuierlich weiterentwickelt werden, um den wechselnden Ansprüchen der Studenten von heute gerecht zu werden. 


\section{LITERATURVERZEICHNIS}

1. Goethe Universität Jahrbuch 2014. Studentische Poliklinik Helfen und Lernen.

https://www.muk.uni-frankfurt.de/58232553/jahresbericht_2014_deu.pdf. Accessed February 9, 2019.

2. Seifert LB, Schaack D, Jennewein L, et al. Peer-assisted learning in a student-run free clinic project increases clinical competence. Med Teach. 2016;38(5):515-522.

3. Seifert LB, Manap A, Reimold P, et al. Die Studentische Poliklinik in Frankfurt. Z Allg Med. 2018;94(11):461-466.

4. StuPoli Hamburg. http://www.stupoli-hamburg.de. Accessed December 16, 2019.

5. Drexler R, Fröschle F, Predel C, et al. Establishing a student-run free clinic in a major city in Northern Europe: a 1-year experience from Hamburg, Germany. J Public Health (Bangkok). December 2019. doi:10.1093/pubmed/fdz165.

6. Medizinische Hochschule Hannover - Patientenversorgung. https://www.mhh.de/allgmed/patientenversorgung. Accessed April 5, 2020.

7. Statistisches Bundesamt. Sozialleistungen - Angaben zur Krankenversicherung (Ergebnisse des Mikrozensus) 2011. Fachserie 13 R 11. 2012.

https://www.destatis.de/GPStatistik/servlets/MCRFileNodeServlet/DEHeft _derivate_00010239/2130110119004.pdf. Accessed February 11, 2019.

8. Schade M, Heudorf U, Tiarks-Jungk P. Die Humanitäre Sprechstunde in Frankfurt am Main: Inanspruchnahme nach Geschlecht, Alter und Herkunftsland. Gesundheitswesen. 2015;77(7):466-474.

9. Wahlfach der Studentischen Poliklinik Frankfurt. Goethe Universität Frankfurt.

https://qis.server.uni-

frankfurt.de/qisserver/rds?state=verpublish\&status=init\&vmfile=no\&publis hid=281575\&moduleCall=webInfo\&publishConfFile=weblnfo\&publishSub Dir=veranstaltung. Accessed December 19, 2019. 
10. Hessisches Ministerium für Wissenschaft und Kunst.

https://wissenschaft.hessen.de/presse/pressemitteilung/hochschulpreisfuer-exzellenz-der-lehre-ehrt-herausragende-lehr-und. Accessed September 29, 2017.

11. Löllgen $\mathrm{H}$. Primärprävention kardialer Erkrankungen: Stellenwert der körperlichen Aktivität. Dtsch Arztebl Int. 2003;100(15):A987-996.

12. Stephens L, Bouvier N, Thomas D, \& Meah Y. Voluntary Participation in a Medical Student-Organized Clinic for Uninsured Patients Significantly Augments the formal Curriculum in Teaching Underrepresented Core Competencies. Journal of Student-Run Clinics, 2015;1(1). https://studentrunfreeclinics.org/journalsrc.org/index.php/jsrc/article/view/ 5. Accessed December 19, 2019.

13. Arenas DJ, Lett LA, Klusaritz H, Teitelman AM. A Monte Carlo simulation approach for estimating the health and economic impact of interventions provided at a student-run clinic. PLoS One. 2017;12(12):e0189718.

14. Society of Student Run Free Clinics. Romoting and supporting the existence of student-run clinics at every health sience center. https://www.student-runfreeclinics.org/. Accessed November 25, 2019.

15. Wikipedia. UCSD Student-Run Free Clinic Project. https://en.wikipedia.org/wiki/UCSD_StudentRun_Free_Clinic_Project\#Nationwide_impact. Accessed March 24, 2019.

16. Beck E. The UCSD Student-Run Free Clinic Project: Transdisciplinary Health Professional Education. J Health Care Poor Underserved. 2005;16(2):207-219.

17. Simpson SA, Long JA. Medical student-run health clinics: Important contributors to patient care and medical education. J Gen Intern Med. 2007;22(3):352-356.

18. Smith S, Thomas R, Cruz M, Griggs R, Moscato B, Ferrara A. Presence and Characteristics of Student-Run Free Clinics in Medical Schools. JAMA. 2014;312(22):2407-2410.

19. Lu KB, Thiel B, Atkins CA, et al. Satisfaction with Healthcare Received at an Interprofessional Student-run Free Clinic: Invested in Training the Next Generation of Healthcare Professionals. Cureus. 2018.e2282 
20. Campbell DJ, Gibson K, O'Neill BG, Thurston WE. The role of a studentrun clinic in providing primary care for Calgary's homeless populations: A qualitative study. BMC Health Serv Res. 2013;13:277.

21. Bureau USC. Health Insurance Coverage in the United States: 2018. https://www.census.gov/library/publications/2019/demo/p60-267.html. Accessed March 24, 2020.

22. Seifer SD. Service-learning: Community-campus partnerships for health professions education. Acad Med. 1998;73(3):272-277.

23. Schutte T, Tichelaar J, Donker E, Richir MC, Westerman M, van Agtmael MA. Clarifying learning experiences in student-run clinics: a qualitative study. BMC Med Educ. 2018;18(1):244.

24. Williams GC, Deci EL. The importance of supporting autonomy in medical education. Ann Intern Med. 1998;129:303-308.

25. Schutte T, Tichelaar J, Dekker RS, van Agtmael MA, de Vries TPGM, Richir MC. Learning in student-run clinics: A systematic review. Med Educ. 2015;22(6):683-698.

26. Lie DA, Forest CP, Walsh A, Banzali Y, Lohenry K. What and how do students learn in an interprofessional student-run clinic? An educational framework for teambased care. Med Educ Online. 2016;21(1):31900.

27. Approbationsordnung für Ärzte. ÄApprO 2002. https://www.gesetze-im-internet.de/_appro_2002/BJNR240500002.html. Accessed March 27, 2018.

28. Ellett JD, Campbell JA, Gonsalves WC. Patient satisfaction in a studentrun free medical clinic. Fam Med. 2010;42(1):16-18.

29. Pons Wörterbuch. Pons Web Site. https://de.pons.com/übersetzung/englisch-deutsch/Peer. Accessed March 11, 2020.

30. Taheri M, Amini M, Delavari S, Bazrafkan L, Mazidimoradi J. Effect of Peer Assisted Learning (PAL) education on knowledge, attitude and behavior related to prevention and control of diabetes. BMC Res Notes. 2019;12(1):227.

31. Boud D, Cohen R, Sampson J. Peer Learning and Assessment. Assess Eval High Educ. 1999;24(4):413-426.

32. Shaterjalali M, Yamani N, Changiz T. Who are the right teachers for 
medical clinical students? Investigating stakeholders\&rsquo; opinions using modified Delphi approach. Adv Med Educ Pract. 2018;9(1):801809.

33. Busari JO, Prince KJAH, Scherpbier AJJA, Van der Vleuten CPM, Essed GGM. How residents perceive their teaching role in the clinical setting: $A$ qualitative study. Med Teach. 2002;24(1):57-61.

34. Whitman NA, Fife JD. Peer Teaching: To Teach Is To Learn Twice. No.4. Washington, D.C.: Association for the Study of Higher Education; 1988.

35. Renkl A. Lernen Durch Lehren: Zentrale Wirkmechanismen beim kooperativen Lernen. Wiesbaden: Deutscher Universitätsverlag; 1997.

36. Lammerding-Köppel M, Fabry G, Hofer M, Ochsendorf F, Schirlo C. Hochschuldidaktische Qualifizierung in der Medizin: I. Bestandsaufnahme. GMS Z Med. 2006;23(4):1-5.

37. Fabry G, Hofer M., Ochsendorf F., Schirlo C., Breckwoldt J., Lammerding-Köppel M.author). Hochschuldidaktische Qualifizierung in der Medizin III: Aspekte der erfolgreichen Implementierung von Qualifizierungsangeboten Ein Positionspapier des GMA-Ausschusses Personal- und Organisationsentwicklung für die medizinische Lehre der Gesellschaft für M. GMS Z Med Ausbild. 2008;25(2):Doc84.

38. Bugaj TJ, Blohm M, Schmid C, et al. Peer-assisted learning (PAL): skills lab tutors' experiences and motivation. BMC Med Educ. 2019;19(1):353.

39. Blohm M, Lauter J, Branchereau S, et al. 'Peer-assisted learning'(Pal) in the skills-lab - an inventory at the medical faculties of the federal republic of Germany. GMS Z Med Ausbild. 2015;32(1):Doc10.

40. Choudhury N, Khanwalkar A, Kraninger J, Vohra A, Jones K, Reddy S. Peer mentorship in student-run free clinics: The impact on preclinical education. Fam Med. 2014;46(3):204-208.

41. Sutkin G, Wagner E, Harris I, Schiffer R. What makes a good clinical teacher in medicine? A review of the literature. Acad Med. 2008;83(1):452-466.

42. Siddiqui S, Siddiqui S, Mustafa Q, Rizvi AF, Hossain IT. The benefits of a peer-assisted mock PACES. Clin Teach. 2018;15(3):221-225.

43. Sobral DT. Peer tutoring and student outcomes in a problem-based course. Med Educ. 1994;28(4):284-289. 
44. Ali N, Anwer M, Abbas J. Impact of Peer Tutoring on Learning of Students. J Stud Manag Plan. 2015;1(2):61-66.

45. Comenius JA. Große Didaktik: Die Vollständige Kunst, Alle Menschen Alles Zu Lehren. 9th ed. (Flitner A, ed.). Stuttgart: Klett-Cotta; 2000.

46. Meah YS, Smith EL, Thomas DC. Student-run health clinic: Novel arena to educate medical students on system-based practice. Mt Sinai J Med. 2009;76(4):344-356.

47. Field M, Burke JM, McAllister D, Lloyd DM. Peer-assisted learning: A novel approach to clinical skills learning for medical students. Med Educ. 2007;41(4):411-418.

48. Tolsgaard MG, Gustafsson A, Rasmussen MB, HØiby P, Müller CG, Ringsted C. Student teachers can be as good as associate professors in teaching clinical skills. Med Teach. 2007;29(6):553-557.

49. Burke J, Fayaz S, Graham K, Matthew R, Field M. Peer-assisted learning in the acquisition of clinical skills: A supplementary approach to musculoskeletal system training. Med Teach. 2007;29(6):577-582.

50. Kononowicz AA, Zary N, Edelbring S, Corral J, Hege I. Virtual patients What are we talking about? A framework to classify the meanings of the term in healthcare education. BMC Med Educ. 2015;15(1):1-7.

51. Association of American Medical Colleges. Effective Use of Educational Technology in Medical Education:Summary Report of the 2006 AAMC Colloquium on Educational Technology. Washington, DC: AAMC 2007.

52. Ellaway R. Modeling Virtual Patients and Virtual Cases Modeling Virtual Patients and Virtual Cases. Whats the Problem? http://meld.medbiq.org/primers/virtual_patients_cases_ellaway.htm. Accessed January 5, 2019.

53. Issenberg SB, McGaghie WC, Petrusa ER, Gordon DL, Scalese RJ. Features and uses of high-fidelity medical simulations that lead to effective learning: A BEME systematic review. Med Teach. 2005;27(1):10-28.

54. Ende J. Feedback in Clinical Medical Education. JAMA J Am Med Assoc. 1983;250(6):777-781.

55. Bienstock JL, Katz NT, Cox SM, Hueppchen N, Erickson S, Puscheck EE. To the point: medical education reviews-providing feedback. Am J 
Obstet Gynecol. 2007;196(6):508-513.

56. Hewson MG, Little ML. Giving feedback in medical education: Verification of recommended techniques. J Gen Intern Med. 1998;13(2):111-116.

57. Kunz K, Burkert M, Heindl F, Schüttpelz-Brauns K, Giesler M. The frequency of using certain feedback methods in the teaching of medicine: A survey of teachers at the medical faculties in baden-wurttemberg. GMS J Med Educ. 2019;36(4):Doc45.

58. Van De Ridder JMM, Stokking KM, McGaghie WC, Ten Cate OTJ. What is feedback in clinical education? Med Educ. 2008;42(2):189-197.

59. Cantillon P, Sargeant J. Giving feedback in clinical settings. BMJ. 2008;337:a1961

60. RKI. Einzelkapitel: Wie haben sich Angebot und Inanspruchnahme der Gesundheitsversorgung verändert? In: Gesundheit in Deutschland. Gesundheitsberichterstattung des Bundes. Robert-Koch-Institut; 2015; 303-374. Doi: 10.17886/rkipubl-2015-003-5

61. Dohmen A, Fiedler M. Ökonomisierung im Gesundheitswesen: Betriebswirtschaftlicher Erfolg als Unternehmensziel. Dtsch Arztebl. 2015;112(9):A364-366 https://www.aerzteblatt.de/archiv/168344/Oekonomisierung-imGesundheitswesen-Betriebswirtschaftlicher-Erfolg-alsUnternehmensziel.\%20Accessed\%20December\%203,\%202019. Accessed December 3, 2019.

62. König S, Stieger P, Sippel S, et al. Train-the-Trainer: Professionalisierung der Lehre im klinischen Alltag - Selbsteinschätzung Lehrender zur didaktischen Kompetenz und den Rahmenbedingungen des Unterrichts. Zentralblatt für Chir. 2019;144(06):551-559

63. Dornan T, Hadfield J, Brown M, Boshuizen H, Scherpbier A. How can medical students learn in a self-directed way in the clinical environment? Design-based research. Med Educ. 2005;39(4):356-364.

64. Marburger Bund. MB-Monitor 2017. https://www.marburger-bund.de/mb-monitor-2017. Accessed January 8, 2019.

65. Schmidt K, Schmidt C, Meyer J, Liebeneiner J. Generation Y Rekrutierung, Entwicklung und Bindung einer neuen Generation von 
Ärzten. DMW - Dtsch Medizinische Wochenschrift. 2012;137(S 03). doi:10.1055/s-0032-1323461

66. Consorti F, Mancuso R, Nocioni M, Piccolo A. Efficacy of virtual patients in medical education: A meta-analysis of randomized studies. Comput Educ. 2012;59(3):1001-1008.

67. Fleetwood J, Vaught W, Feldman D, Gracely E, Kassutto Z, Novack D. MedEthEx Online: A Computer-Based Learning Program in Medical Ethics and Communication Skills. Teach Learn Med. 2000;12(2):96-104.

68. Krüger-Brand HE. E-Learning in der Medizin vor dem Durchbruch. Dtsch Arztebl. 2002;99(22):A1491-1493.

https://www.aerzteblatt.de/archiv/31832/E-Learning-in-der-Medizin-Vordem-Durchbruch. Accessed 15 January, 2020.

69. Tworek J, Coderre S, Wright B, McLaughlin K. Virtual patients: ED-2 band-aid or valuable asset in the learning portfolio? Acad Med. 2010;85(1):155-158.

70. Bryce DA, King NJ, Graebner CF, Myers JH. Evaluation of a Diagnostic Reasoning Program (DxR): Exploring Student Perceptions and Addressing Faculty Concerns. J Interact Media Educ. 1998;98(1):1-34.

71. Cook DA, Erwin PJ, Triola MM. Computerized Virtual Patients in Health Professions Education: A Systematic Review and Meta-Analysis. Acad Med. 2010;85(10):1589-1602.

72. Gormley GJ, McGlade K, Thomson C, McGill M, Sun J. A virtual surgery in general practice: Evaluation of a novel undergraduate virtual patient learning package. Med Teach. 2011;33(10):e522-527.

73. Ellaway RH, Poulton T, Smothers V, Greene P. Virtual patients come of age. Med Teach. 2009;31(8):683-684.

74. Janda MS, Mattheos N, Nattestad A, et al. Simulation of patient encounters using a virtual patient in periodontology instruction of dental students: Design, usability, and learning effect in history-taking skills. Eur J Dent Educ. 2004;8(3):111-119.

75. Kumta SM, Tsang PL, Hung LK, Cheng JCY. Fostering critical thinking skills through a web-based tutorial programme for final year medical students- A randomized controlled study. J Educ Multimed Hypermedia. 2003;12(3):267-273. 
76. Higgs J, Jensen GM, Loftus S, Christensen N. Clinical Reasoning in the Health Professions. ElsevierHealth Sciences; 2018.

77. Hege I, Kononowicz AA, Berman NB, Lenzer B, Kiesewetter J. Advancing clinical reasoning in virtual patients - development and application of a conceptual framework. GMS J Med Educ. 2018;35(1):Doc12

78. Lateef F. Clinical Reasoning: The Core of Medical Education and Practice. Intern Emerg Med. 2018;1(2):1015.

79. Botezatu M, Hult H, Tessma MK, Fors U. Virtual patient simulation: Knowledge gain or knowledge loss. Med Teach. 2010;32(7):562-568.

80. Kandasamy T, Fung K. Interactive Internet-based cases for undergraduate otolaryngology education. Otolaryngol - Head Neck Surg. 2009;140(3):398-402.

81. Kerfoot BP, Baker $\mathrm{H}$, Jackson TL, et al. A multi-institutional randomized controlled trial of adjuvant Web-based teaching to medical students. Acad Med. 2006;81(3):224-230.

82. Klemme B, Siegmann G. Clinical Reasoning: Therapeutische Denkprozesse Lernen. 2nd ed. Stuttgart: Georg Thieme Verlag; 2014.

83. Amey L, Donald KJ, Teodorczuk A. Teaching clinical reasoning to medical students. Br J Hosp Med. 2017;78(7):399-401.

84. Deladisma AM, Cohen M, Stevens A, et al. Do medical students respond empathetically to a virtual patient? Am J Surg. 2007;193(6):756-60.

85. Vash JH, Yunesian M, Shariati M, Keshvari A, Harirchi I. Virtual patients in undergraduate surgery education: A randomized controlled study. ANZ J Surg. 2007;77(1-2):54-59.

86. Learning Resource Server Medizin. LRSMed Web site. http://www.Irsmed.de/index.xsql. Accessed November 19, 2017.

87. Stausberg J, Kühnl A. E-Learning in der Medizin: Erweitertes Angebot. Dtsch Arztebl. 2013;110(37):A1692 https://www.aerzteblatt.de/archiv/145856/E-Learning-in-der-MedizinErweitertes-Angebot. Accessed November 19, 2017.

88. INMEDEA Simulator. http://www.inmedea-simulator.net/med/scene/entry? Accessed December 22, 2019. 
89. CompuGroup Medical Deutschland AG. TELEMED Website.

https://www.cgm.com/de/arzt_zahnarzt/it_infrastruktur_de/telemed/telem ed_online_loesungen/telemed_inmedea_simulator/der_inmedea_simulat or.de.jsp. Accessed December 22, 2019.

90. CompuGroup Medical Deutschland AG. Anwenderbericht zur CME Fortbildung mit dem INMEDA Simulator. https://www.cgm.com/media/cgm_de/documents/products__solutions_1 2/physicians_1/telemed/datenblaetter/inmedea/2015_13_11/2015-1113_telemed_Inmedea_Anwenderbericht_LAeKBaWue_Rev1_0_DE.pdf Accessed December 22, 2019.

91. Goethe-Universität. Studium digitale Website. https://www.studiumdigitale.uni-frankfurt.de/59593095/LernBar-2015? Accessed January 13, 2020.

92. LernBar-Portal. LernBar Website. https://lernbar.uni-frankfurt.de/. Accessed January 13, 2020.

93. Goethe-Universität. Prüfungsverwaltung Fachbereich 16. https://www.unifrankfurt.de/59951446/Prüfungsverwaltung_Fachbereich_16. Accessed February 3, 2019.

94. Harden RM, Downie WW, Stevenson M, Wilson GM. Assessment of Clinical Competence using Objective Structured Examination. Br Med J. 1975;1(5955):447-451.

95. Harden RM, Gleeson FA. Assessment of clinical competence using an objective structured clinical examination (OSCE). Med Educ. 1979;13(1):41-54.

96. Microsoft Office 365 (for Mac) [computer program]. Version 16.35. Redmond, WA: Microsoft Corp.; 2020

97. Institut für Medizinische und Pharmazeutische Prüfungsfragen. IMPP Website.

http://impp.de/start.html. Accessed February 3, 2019.

98. Goethe-Universität. Kursabfolge im klinischen Studium. https://www.uni-frankfurt.de/72609594/Studienverlauf.pdf. Accessed February 16, 2020.

99. Goethe-Universität. Fächerlisten Semesterabschlussklausuren. 
https://www.uni-

frankfurt.de/74834668/Faecherliste_gesamt_ab_WS1819.pdf.

Accessed February 16, 2020.

100. Google Formulare: Kostenlos Umfragen erstellen und analysieren. https://www.google.de/intl/de/forms/about/. Accessed February 5, 2019.

101. Brust FG. Fachbereich Medizin Logbuch für das Praktische Jahr Wahlfachtertial: Allgemeinmedizin.

https://www.uni-

frankfurt.de/69554441/PJ_Logbuch_Allgemeinmedizin_Version_6_0.p Accessed February 19, 2020.

102. GraphPad Prism (for Mac) [computer program]. Version 7.0. San Diego, CA: GraphPad Software; 2018

103. Methodenberatung Universität Zürich. UZH Web Site. https://www.methodenberatung.uzh.ch/de.html. Accessed March 24, 2020

104. Irby DM, Ramsey PG, Gillmore GM, Schaad D. Characteristics of effective clinical teachers of ambulatory care medicine. Acad Med. 1991;66(1):54-55.

105. Raupach T, Brown J, Anders S, Hasenfuss G, Harendza S. Summative assessments are more powerful drivers of student learning than resource intensive teaching formats. BMC Med. 2013;11(5):61.

106. Larsen DP, Butler AC, Roediger HL. Test-enhanced learning in medical education. Med Educ. 2008;42(10):959-966.

107. Roediger HL, Karpicke JD. The Power of Testing Memory: Basic Research and Implications for Educational Practice. Perspect Psychol Sci. 2006;1(1):181-210.

108. Kerres M, Witt $\mathrm{C}$ de, Stratmann J. E-Learning. Didaktische Konzepte für erfolgreiches Lernen. In Schwuchow K, Guttman J eds. Jahrbuch Personalentwicklung \& Weiterbildung 2003. Neuwied:Luchterhand Verlag. 2002.1-14.

109. Karimi Mouneghi H, Derakhshan A, Vlai N, Mortazavi F. The effectiveness of video-based education on gaining practical learning skills in comparison with demonstrating method's effectiveness among university students. J Med Educ. 2003;4(1):27-30. 
110. Devi B, Khandelwal B, Das M. Comparison of the effectiveness of videoassisted teaching program and traditional demonstration on nursing students learning skills of performing obstetrical palpation. Iran J Nurs Midwifery Res. 2019;24(2):118-123.

111. Tayade A, Tayade S, Chalak A, Srivastava T. The impact of Video Assisted learning (VAL) on slow learners. Int $J$ Biomed Adv Res. 2018;9(1):13-18.

112. Schwerdtfeger K, Wand S, Schmid O, et al. A prospective, blinded evaluation of a video-assisted "4-stage approach" during undergraduate student practical skills training. BMC Med Educ. 2014;14(1):104.

113. Shumway JM, Harden RM. AMEE Guide No.25: The assessment of learning outcomes for the competent and reflective phyicia. Med Teach. 2003;25(6):569-584.

114. Van Der Vleuten CPM. The assessment of professonal competence: Developments, research and practical implications. Adv Heal Sci Educ. 1996;1(1):41-67.

115. Wormald BW, Schoeman S, Somasunderam A, Penn M. Assessment drives learning: An unavoidable truth? Anat Sci Educ. 2009;2(5):199-204.

116. Kromann CB, Jensen ML, Ringsted C. The effect of testing on skills learning. Med Educ. 2009;43(1):21-27.

117. Haist SA, Witzke DB, Quinlivan S, Murphy-Spencer A, Wilson JF. Clinical skills as demonstrated by a comprehensive clinical performance examination: Who performs better - Men or women? Adv Heal Sci Educ. 2003;8(3):189-199.

118. Ferguson E, James D, Madeley L. Factors associated with success in medical school: Systematic review of the literature. Br Med J. 2002;324(7343):952-957.

119. Woolf K, Haq I, McManus IC, Higham J, Dacre J. Exploring the underperformance of male and minority ethnic medical students in first year clinical examination. Adv Heal Sci Educ. 2008;13(5):607-616.

120. Haq I, Higham J, Morris R, Dacre J. Effect of ethnicity and gender on performance in undergraduate medical examinations. Med Educ. 2005;39(11):1126-1128.

121. Archer JC. State of the science in health professional education: 
Effective feedback. Med Educ. 2010;44(1):101-108.

122. Ruesseler M, Sterz J, Bender B, Hoefer S, Walcher F. The effect of video-assisted oral feedback versus oral feedback on surgical communicative competences in undergraduate training. Eur J Trauma Emerg Surg. 2017;43(4):461-466.

123. Strauss S. Learning Theories of Gagne and Piaget: Implications for Curriculum Development. Teach Coll Rec. 1972;74(1):81-102.

124. Benedict N. Virtual patients and problem-based learning in advanced therapeutics. Am J Pharm Educ. 2010;74(8).143-148. 
10 ANHANG

10.1 Abkürzungsverzeichnis

Abb. Abbildung

OSCE Objective Structured Clinical Examination

PAL Peer-assisted Learning

SP Studentische Poliklinik

Tab. Tabelle

UkliF Untersuchungskurs der klinischen Fächer

VPL Virtual Patients Learning 


\subsection{Tabellenverzeichnis}

Tabelle 1 Vorteile und Nachteile von virtuellen Patienten

Tabelle 2 Lernziele der einzelnen Wahlfachmodule der Studentischen Poliklinik

Tabelle 3 Übersicht der für die Leistungsmessung genutzten Tests

Tabelle 4 Altersverteilung der Studiengruppen

Tabelle 5 Vergleich von Vortest und Nachtest in den einzelnen Studiengruppen

(PAL, VPL und KG) nach erfolgter thematischer Unterteilung und Item Analyse der bearbeiteten Testfragen

Tabelle 6 Vergleich von PAL, VPL und KG untereinander im Vortest und Nachtest

Tabelle 7 Vergleich der Kurztestergebnisse zwischen PAL und VPL

Tabelle 8 Ergebnisse im Wahlfach OSCE

Tabelle 9 Ergebnisse im Curricularen OSCE

Tabelle 10a Selbsteinschätzung vorher und nachher in der PAL Gruppe, VPL Gruppe und KG - Untersuchung

Tabelle 10b Selbsteinschätzung vorher und nachher in der PAL Gruppe, VPL Gruppe und KG - Kommunikation

Tabelle 10c Selbsteinschätzung vorher und nachher in der PAL Gruppe, VPL Gruppe und KG - Klinisch-praktische Fertigkeiten

Tabelle 10d Selbsteinschätzung vorher und nachher in der PAL Gruppe, VPL Gruppe und KG - Klinisches Denken

Tabelle 104 Selbsteinschätzung vorher und nachher in der PAL Gruppe, VPL Gruppe und KG - Therapie

Tabelle 11a Seminarevaluationen der PAL Gruppe und VPL Gruppe (Mediane und $p$-Werte)

Tabelle 11b Seminarevaluationen der PAL Gruppe und VPL Gruppe (Mediane und $p$-Werte) 


\subsection{Abbildungsverzeichnis}

Abbildung $1 \quad$ Studiendesign

Abbildung 2 Einführende Folie zum PAL Seminar Bauchschmerz

Abbildung $3 \quad$ Weiterführende Diagnostik im PAL Seminar Bauchschmerz"

Abbildung $4 \quad$ Folie zur Therapie im PAL Seminar Bauchschmerz

Abbildung 5 e-Learning Plattform Lernbar-Kasuistiken

Abbildung $6 \quad$ Einführung in das VPL Seminar Bauchschmerz

Abbildung $7 \quad$ Freitextaufgabe aus dem VPL Seminar Bauchschmerz

Abbildung $8 \quad$ Angegebene Falschantwort mit dazugehöriger Erklärung im VPL Seminar Bauchschmerz

Abbildung $9 \quad$ Ultraschallbild Abdomen im VPL Seminar Bauchschmerz Abbildung 10 Überblick Wahlfach und Leistungsmessung mit Zeitverlauf Abbildung $11 \quad$ Vergleich aller Studiengruppen im Vortest und Nachtest Abbildung 12 Ergebnis aller Kurztests im PAL Seminar und VPL Seminar Abbildung 13 Ergebnisse im Curricularen OSCE 
10.4 Virtual Patient Learning Seminare

Kasuistik Anhaltender Husten

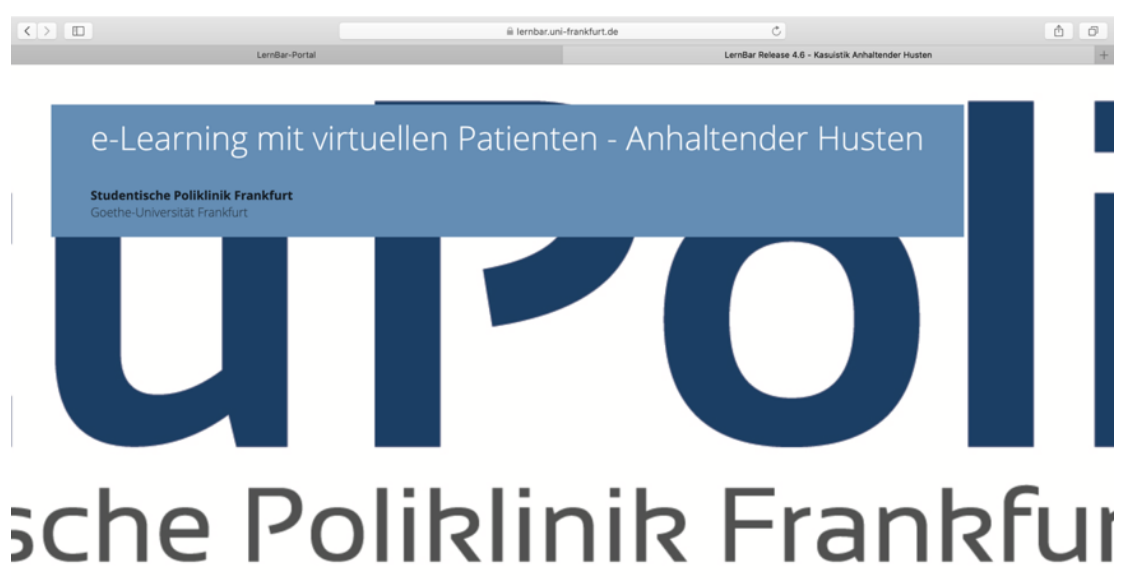

\section{Goethe Universität KURS STARTEN}

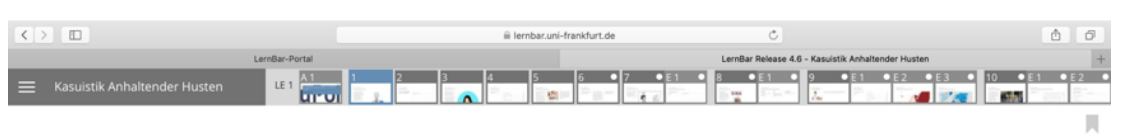

Kasuistik - Anhaltender Huste

Thema: Anhaltender Husten

Hallo, in dieser Online- Kasuistik wirst du

dich mit dem Leitsymptom Husten

Geschattgen. Zunachst bekommst du ein

Farbeispiet vorgestellitund anhand dieses

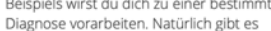

jede Menge Informationen und zu

Fragen die dir cabe

Bild

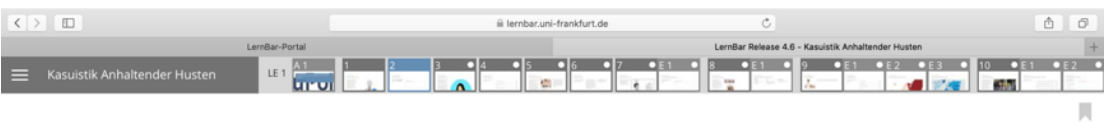

Kasuistik- Anhaltender Husten
Persönliche Daten

Bitte schreibe in das Feld deinen Vor-und
Namen und deine Matrikelnummer 


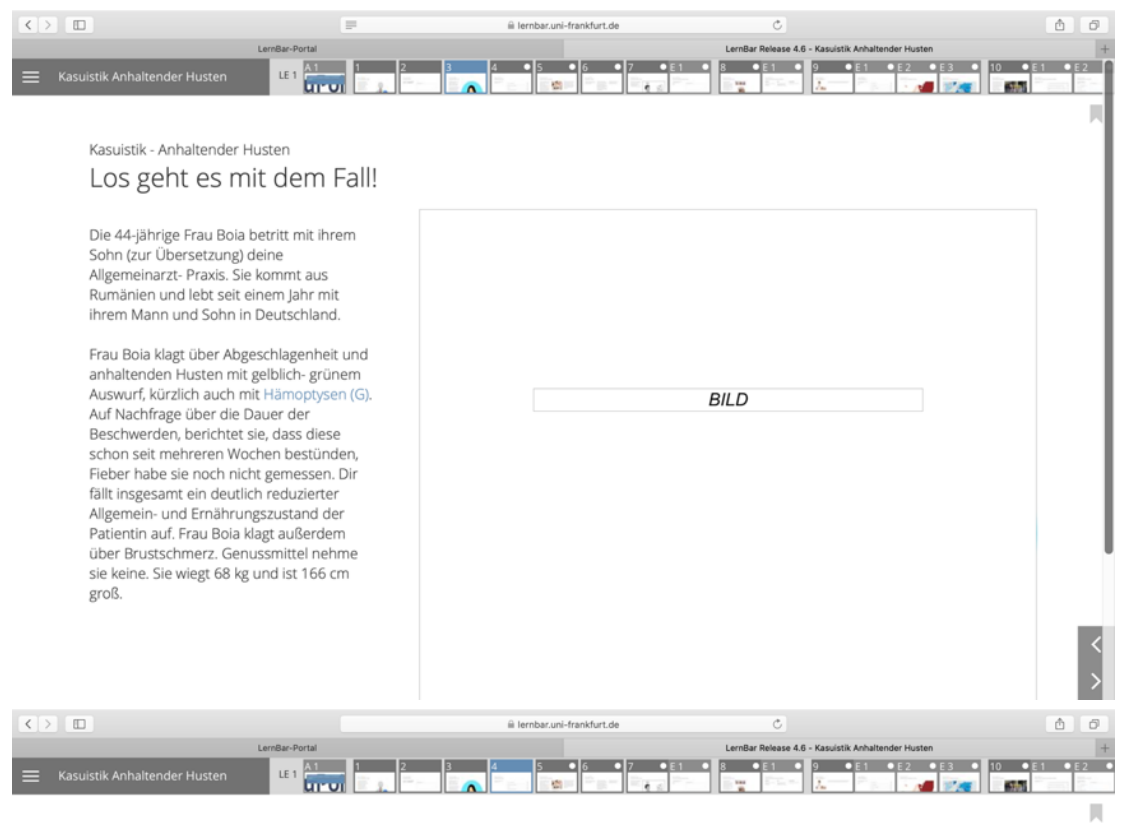

Kasuistik - Anhaltender Husten

Erweiterte Anamnese

Was solltest du in deiner Anamnese

unbedingt noch fragen?

\begin{tabular}{ll} 
1. psychische Erkrankungen & $\square$ \\
\hline 2. Vorerkrankungen und Medikamente & $\square$ \\
\hline 3. Allergien & $\square$ \\
\hline 4. NachtschweiB und Gewichtsverlust & $\square$
\end{tabular}

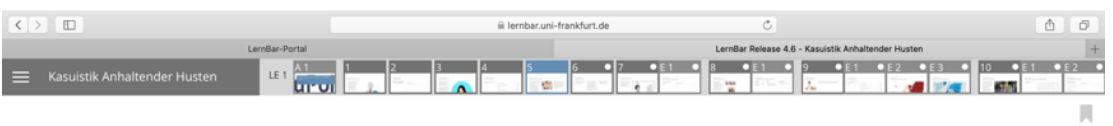

Kasuistik - Anhaltender Huster

Ergebnisse deiner Anamnese

Sehr gut. Du solltest auf jeden Fall noch nach Nachtschweiiß und Gewichtsverlus fragen, um so die B-Symptomatik

(G)ollständig zu erheber

Die B-Symptomatw ist also eine wichtige

Symptomentrias bestenend aus

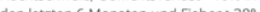

ist typisch bei Morbus. Hodgkin Non-

Hodgkin- Lymphomen und anderen

Malignomen, kommt aber auch vor bei der

Tuberkulose und bei entzündlichen

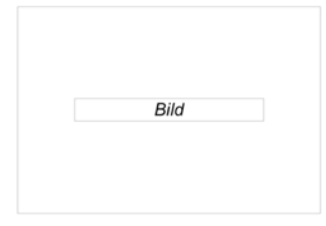

Folgendes konntest du also herausfinden:

teinen Gewichtsverlust von

$\mathrm{kg}$ in 4 Monaten gehabt, sie hat keinen

Vorerkrkrankungen leidet sie unter Diabetes

mellitus Typ 2 und einer Hypothyreose

immt Metformin und L-Thyroxin

Auch die Frage nach Allergien,

Medilamenteneinnahme ist sehr wichtig

Sie können Hinweise auf die vorliegende

Symptomatik geber 


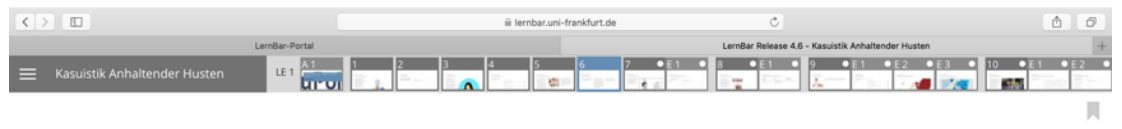

Kasuistik - Anhaltender Huste

Klassifikation Husten

Je nach Dauer des Hustens spricht mar von akutem Husten (<8 Wochen) oder von chronischem ( $>8$ Wochen)

Eine weitere Einteilung erfolgt nach Produktivem oder unproduktivem Husten. man, wenn der Patient Auswurf hat.

welchen man genauer differenzieren kann

Weili-glasiger Auswur deutet z. B. auf ein

virale Infektion hin, gelblich- grune

Auswurt eher auf eine bakterielle Infektion
Frau Boia hat also zusammenfassend einen

(blutiges Aushusten)

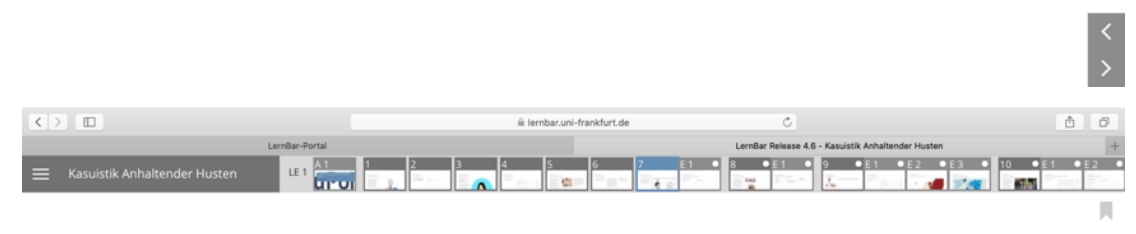

Kasuistik - Anhaltender Husten

Körperliche Untersuchung

Nach der gründlichen Anamnese beginnst

du mit der korperilichen Untersuchun

konzentrieren? Welche Untersuchungen

stehen im Vordergrund?

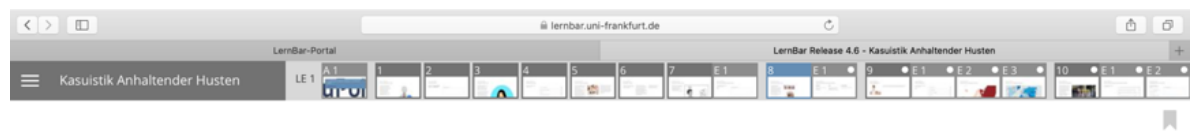

Kasuistik - Anhaltender Husten

Körperliche Untersuchung

Unser Augenmerk liegt auf der

Untersuchung der Lunge.

Irotzdem solite man eine vollständige

korpertiche Untersuchung durchfuhren.

abdominelle Untersuchung und die

Hier ist ein Lehnideo zur

Lungenuntersuchung (mmow) eingefügt.

Schaue es dir ganz genau an.

Da wir eine Patientin haben, die mit dem Leitsymptom Husten kommt, solltest du

auch einmal die wichtigsten

Lymphknotenstationen ( $(w w w)$

untersuchen. Duachtest dabei auf: Große

erschieblichkeit, Schmerz Konsistenz.

Bild

Bild 


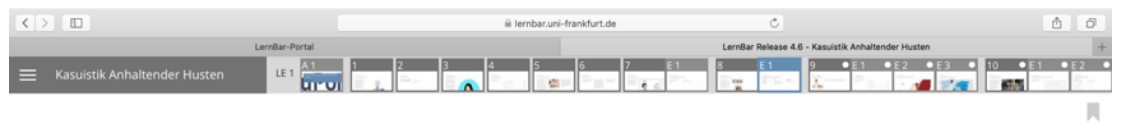

Kasuistik - Anhaltender Husten

Ergebnisse der körperlichen Untersuchung

Vitalparamter

Blutdruck beidseits bei $110 / 70 \mathrm{mmHg}$ Temperatur $38.8^{\circ} \mathrm{C}$
Pulmo

beidseits vesikuläres Atemgeräusch, Rasselgeräusche rechts basal, hyposonoret
Lymphnotenstatus

keine vergrößerten Lymphknoten tastbar

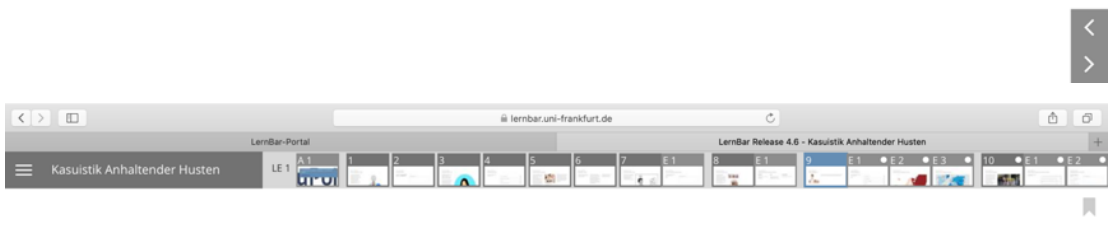

Kasuistik - Anhaltender Husten

Differentialdiagnosen
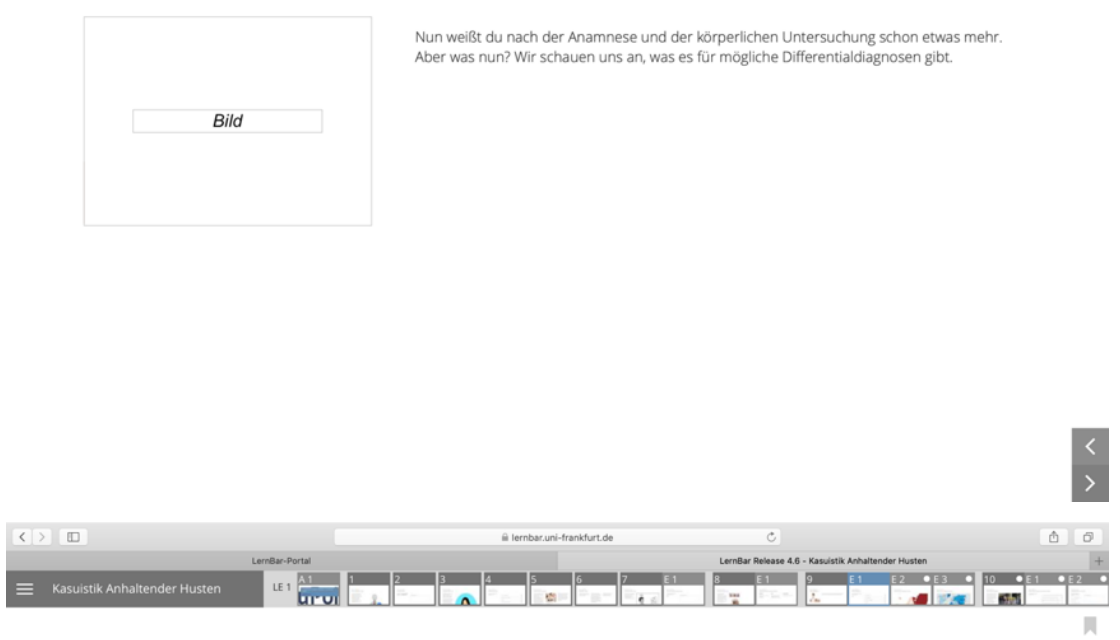

Kasuistik - Anhaltender Husten

Differentialdiagnosen

Was denkst du gehört zu den möglichen

VTipp

\begin{tabular}{lc} 
1. Bronchial-Carcinom & $\square$ \\
\hline 2. Akuter Atemwegsinfekt & $\square$ \\
\hline 3. ACE-Hemmer-Unverträglichkeit & $\square$ \\
\hline 4. Tuberkulose & $\square$ \\
\hline 5. COPD & $\square$ \\
\hline 6. Malignes Lymphom & $\square$
\end{tabular}

Denknocheinma

der körperlichen Untersuchung nach. Auch

die Anamnese kann Hinweise geben.

Malignes Lymphom

$\square$ 


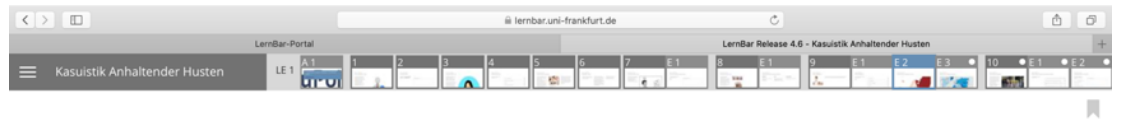

Kasuistik - Anhaltender Husten

Vorkommen von Tuberkulose in Europa

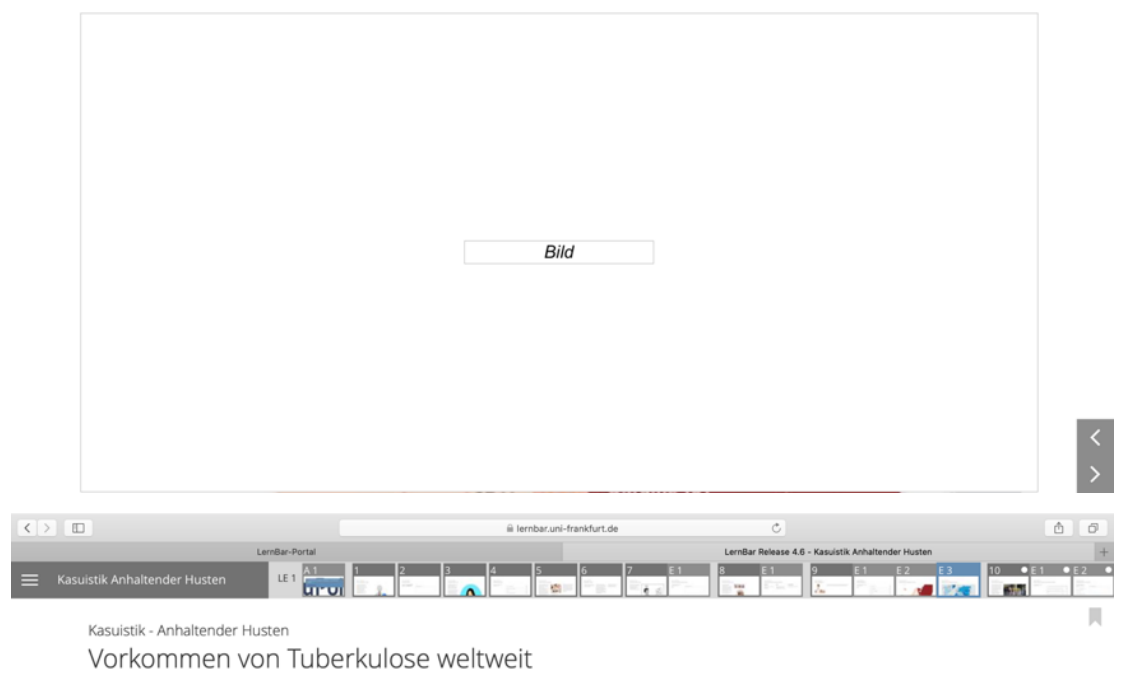

Vorkommen von Tuberkulose weltweit

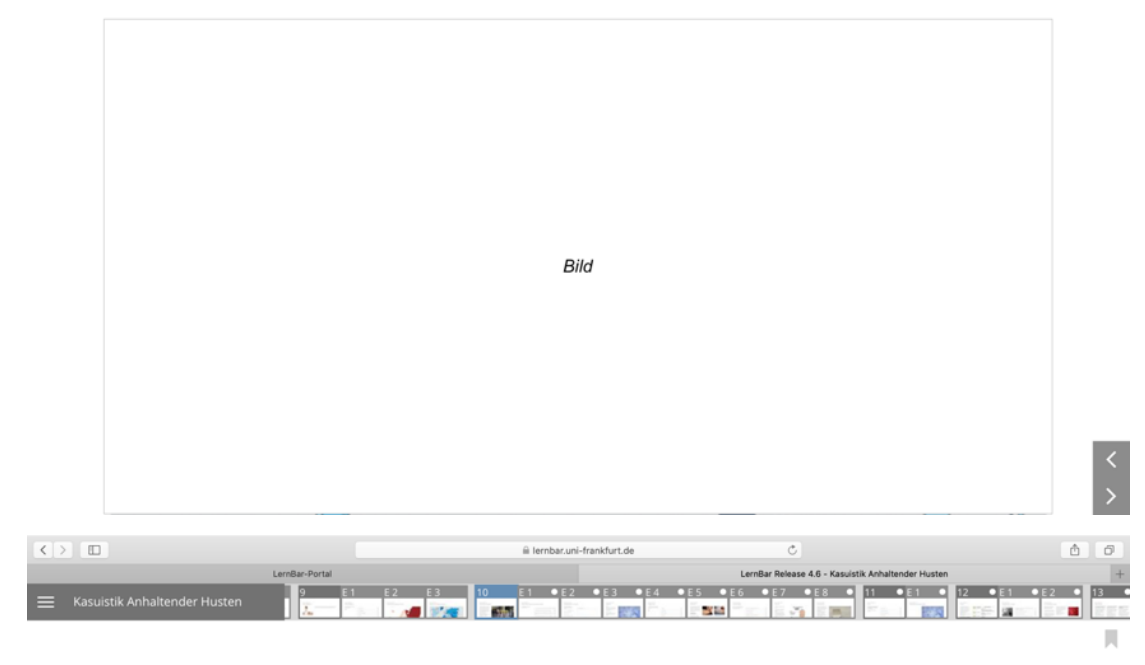

Kasuistik - Anhaltender Husten

Weiterfrührende Diagnostik

Nun sind wir soweit, dass wir mehrere

Diterentialdiagnosen kennen. Anamnese

hindeuten Wichtig ist doss wir an eine

"Tbc" (-Tuberkulose) denken! of kann

diese zunächst beschwerdeffei verlaufen

oder eine uncharakteristische Symptomatix

Uuberlege nun weiche Diagnostik du weiter

einleitest um deinen Verdacht zu

Bild

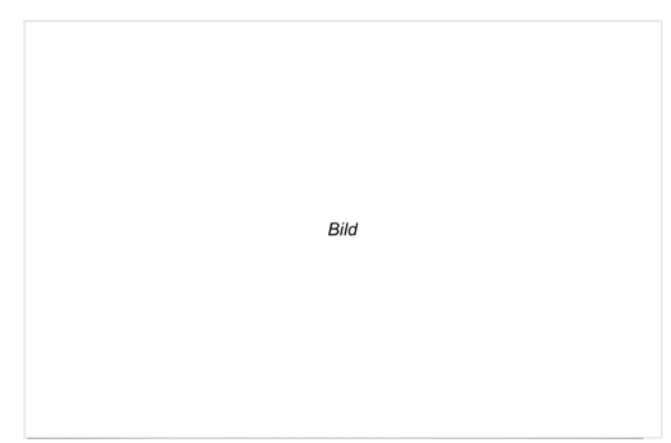




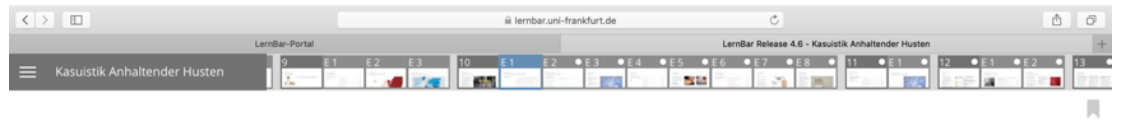

Kasuistik - Anhaltender Husten

Welche Untersuchungen sind als nächstes indiziert?

Welche diagnostischen Methoden

möchtest du einleiten?

\begin{tabular}{l} 
1. Ich führe eine Blutentnahme durch und lasse zusätzlich eine Blutkultur machen. \\
\hline 2. Ein Röntgen- Thorax leite ich neben einer Sputumuntersuchung ein. \\
\hline $\begin{array}{l}\text { 3. Tuberkulin- Hauttest und Quantiferontest dürfen in meiner Diagnostik nicht } \\
\text { fehlen. }\end{array}$ \\
\hline 4. Ich sonographiere die Lunge und leite eine Stuhl- und Urin- Diagnostik ein. \\
\hline 5. Sobald der Verdacht auf Tbc besteht, leite ich den Patienten an einen Spezialisten \\
(Infektiologe) weiter.
\end{tabular}

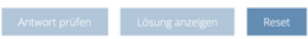

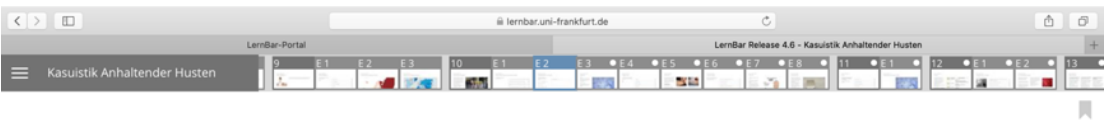

Kasuistik - Anhaltender Husten

Auffällige Blutwerte von Frau Boia

Folgende Ergebnisse zeigt die

Blutuntersuchung (Referenzwerte in

Klammern).

Hämoglobin (G) 105 g/l (136-172 g/l)

Erythrozyten 3,8/pl (4,3-5,9/pl)

MCV 75 กी (81-100 ff

Leukozyten 3800//1 (4000 - 10000/w)

CRP $31 \mu g /(<5 \mu g /)$

Außerdem ist die BSG

(Blutsenkungsgeschwindigkeit) etwas

Wie interpretiertst du diese Werte?
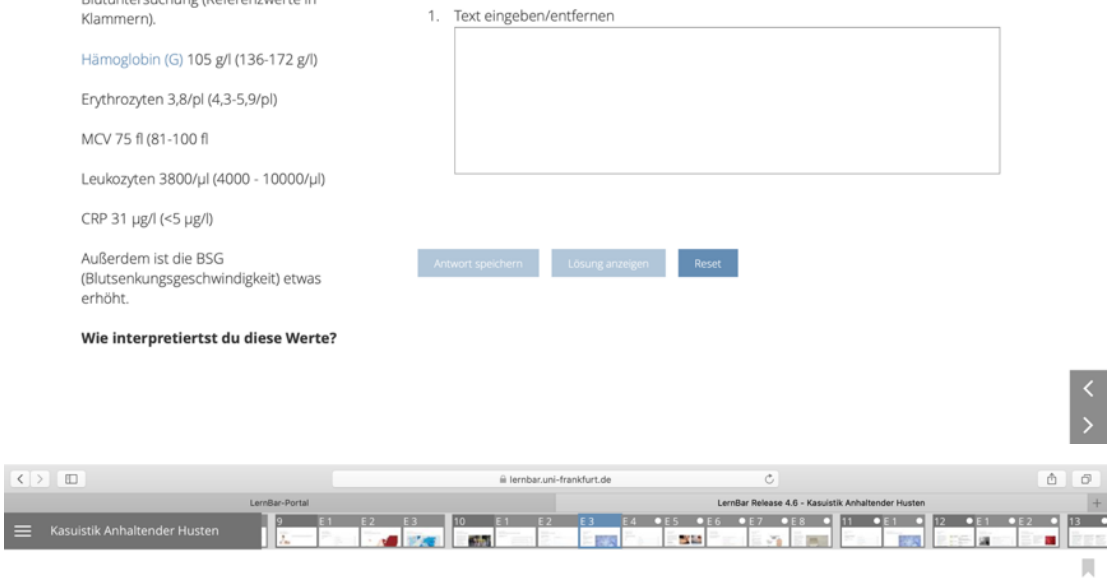

Kasuistik - Anhaltender Husten

Ergebnisse

Blutbild:

Patientin hat eine mikrozyatre

Anamie, einen leicht erhöhten CRP. Wer

eukozyopenie.

Blutkultur und Sputum

Direkte Erregernachweise aus Sputum

(Mikroskople und Kultur) sind positi 


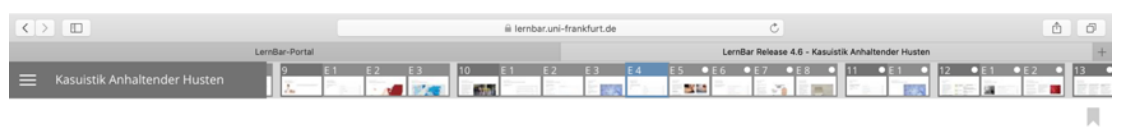

Kasuistik - Anhaltender Husten

Röntgen-Thorax

Hier siehst du nun das a.p. Röntgenbild

Du siehst sogenannten Kavernen in beider

Lungen.

\begin{tabular}{lc} 
1. Emphysem & 0 \\
\hline 2. Lungen-Carcninom & 0 \\
\hline 3. Offene Tuberkulose & 0 \\
\hline 4. Lungenzysten & 0 \\
\hline 5. Lungenabszess & 0
\end{tabular}

Wie lautet deine Diagnose?

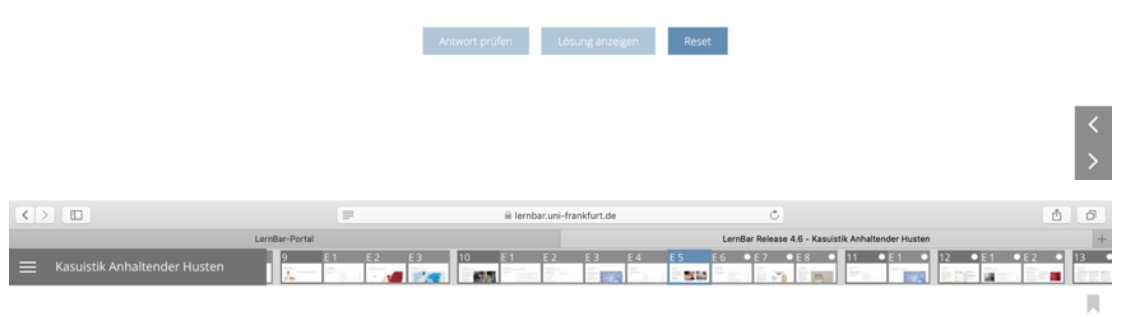

Kasuistik - Anhaltender Husten

Weitere Tests

Nachfolgend werden zwei Tests vorgestelt Beide Tests sind spezifische Test zum

Nachweis der Foc thetekion, Jedoch ist eine aktive von einer latenten Erkrankung unterscheiden kanr.

Tuberkulin-Hauttest
(nach Mendel-Mantoux, THT):

Intrakutan werden in den Unterarm

(Beugeseite) abgewandete Tuberkutin-

Proteine inizizert. Nach 48 - 72 Stunden wird

der Durchmesser der Induration an der

markierten Injektionsstelle beurteilt $(\tau$ - Zell.

Reaktion auf das Antigen). Indurationen

uber >

indurationen $>15 m$ mals hochpositiv (t)

mit Blasenbildung bewertet. Es wird nur
die Induration und nicht die Hautrötung

beurteilt
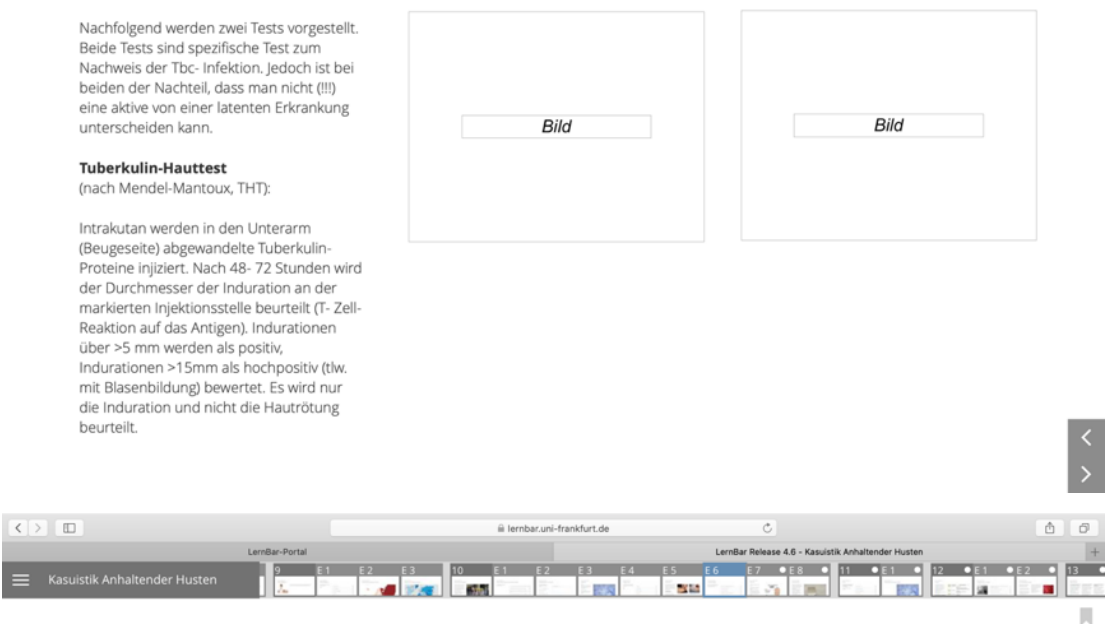

Kasuistik - Anhaltender Husten

Tuberkulin-Hauttest

Beim THT ist nur eine geringe Sensitivita

für falsch-n negative Werte sein?

\begin{tabular}{lc} 
1. Infektion mit M. tuberkulosis liegt 3 Wochen zurück & O \\
\hline 2. Immunsuppression (z.B. HIV) & 0 \\
\hline 3. Einnahme von Antibiotika & 0 \\
\hline 4. Desinfektion des Hautareals vor Testdurchfürung & 0 \\
\hline 5. Superinfektion mit Streptokokken & 0
\end{tabular}

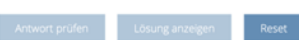




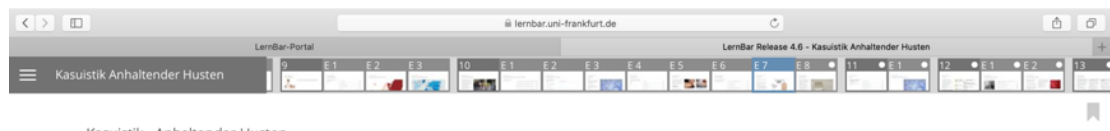

Ittender Husten

Exkurs HIV

HIV kann man als Risikofaktor für die TbC betrachten:

Wettweit betrachtet ist Tuberkulose die

Túnerte Todesursache bera

wechselseitig den Krankheitsverlauf Vor

allem spielt die Koinzidenz von HIV und Tbe

in den 3. Welt- Ländern eine wichtige Rolle

und sollte nicht unterschätzz werder.

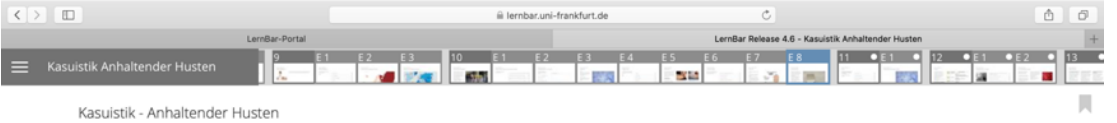
Kasuistik - Anhaltender Husten
Quantiferon-Test

Bei diesem Test wird mittels EUSA die

Menge an Interferon-y gemessen, die von
den T-Zellen des Patienten bei Kontaks mit

Antigenen des Tuberkuloseerregers

ausgeschüttet wird.

Hierfür erfolgt eine Blutentnahme in 3

speziellen Röhrchen. Zeigt sich im

Testrohrchen ein erhonter wert is

Auseinandersetzung mit Tuberkylose und

somit fưr eine Infektion.

Vorteil ist bei diesem Test, dass es eine

hōhere Sensitvităta als der Tuberkulin-

Hauttest hat.

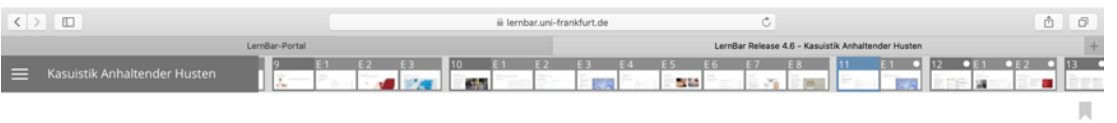

Kasuistik - Anhaltender Huster

Exkurs

Hier ein kleiner Einschub zu den Erregern

haben.

Welche sind die Haupterreger der

Tuberkulose?

\begin{tabular}{ll} 
1. Mycobacterium bovis & 0 \\
\hline 2. Mycobacterium tuberculosis & 0 \\
\hline 3. Bacillus Calmette-Guérin (BCG) & 0 \\
\hline 4. Viridae tuberculosis & 0 \\
\hline 5. diverse gramnegative Stäbchen & 0
\end{tabular}

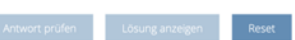




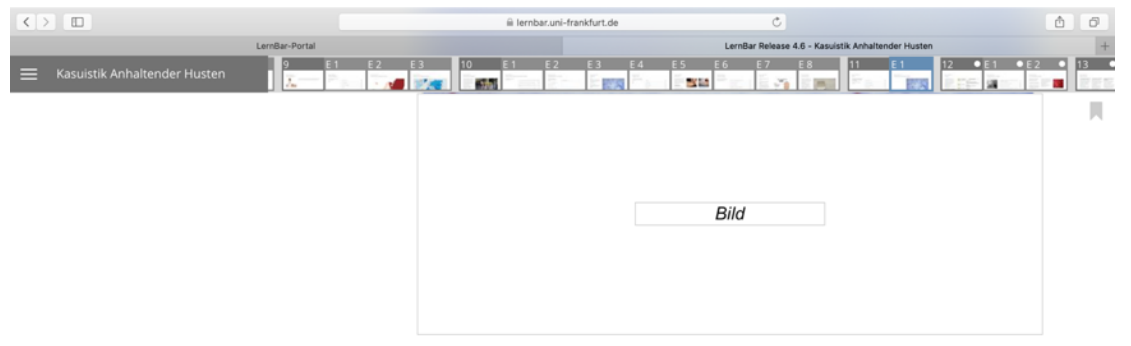

Die verschiedenen Unterklassen der Tuberkulose-Erreger werden zum Mycobacterium. uberculosis-Komplex zusammengefasst. Mykobakterien sind grampositive säurefeste aerobe Der Haupterreger (>95\%) ist dabei M. tuberculosis.

M. tuberculosis Komplex:

M. tuberculosis

M. bovis

M. africanum
M. canetti

M. microt

M. mungi

M. pinnipedi (nicht menschenpathogen)

Bacillus Calmette-Guerin (BCG-Impistamm, stammt von M. bovis ab, löst keine Tbc aus, wird trotzdem zum Komplex gezăh t'

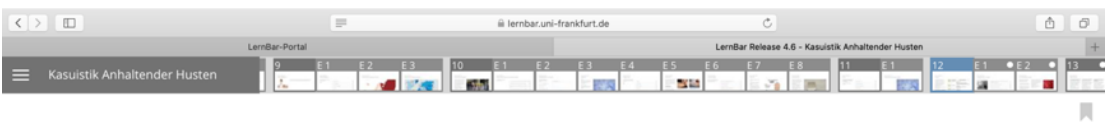

Kasuistik - Anhaltender Husten

Therapie

Es ist eine Standardtherapie für die unkomplizierte Toc vorhanden, die eine versieht Du kannst dind. 6 Monaten Antituberkulotika mit "PREIS" oder mit "in Russland steht ein Panzer" "jeder

Anfangsbuchstabe stent fur en

ungen müssen isoliert

Standardtherapie der unkomplizierten

Tbc bei Erwachsenen:

Das Therapieschema sieht für die ersten 2

Monate eine Kombination von Isoniazid -

Die Wein t pyrazinamid t thamoutor

Gabe von Isoniazid und Pifampicin D

musst nicht die komplette Tabelle mit den

Nebenwirkungen können, aber mindestens

die Wirkstoffe für die Standardtherapie

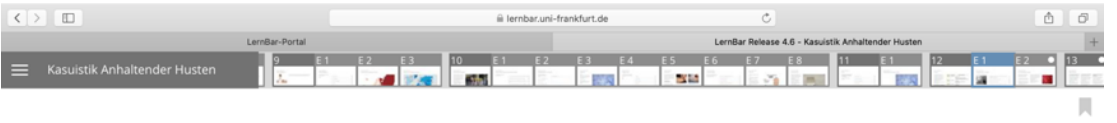

Kasuistik - Anhaltender Husten

Was gehört noch zur Therapie?

Was darfst du auf keinen Fall vergessen?

Bild

\begin{tabular}{lc}
$\begin{array}{l}\text { 1. Jeder Patient, der mit Tbc infiziert ist, muss für } 6 \text { Monate isoliert in einem } \\
\text { Krankenhaus behandelt werden. }\end{array}$ & 0 \\
\hline 2. Namentliche Meldung an das Gesundheitsamt & 0 \\
\hline 3. Mitbehandlung aller Kontaktpersonen! & 0 \\
\hline 4. Mitbehandlung von "mir selbst", da ich den Patienten untersucht habe. & 0
\end{tabular}




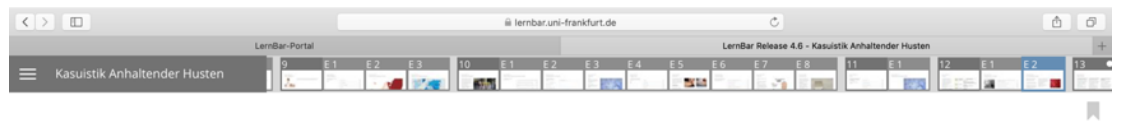

Kasuistik - Anhaltender Husten

Cave: Multiresistente Erreger

Single-drug-resistant Tuberculosis (SDR-TB):

gegen Isoniazid (INH) oder(l) Rifampicin

Multi-drug-resistant Tuberculosis (MDR-TB):

gegen INH und() RMP resistenter Keim

Mōgliche Gründe dafür sind eine

$\begin{array}{ll}\text { Extensively-drug-resistant Tuberculosis } & \begin{array}{l}\text { Werkstoffkonzentration, } 2 . B \text {. be } \\ \text { mangelnder Compliance oder nich }\end{array}\end{array}$

Winerchinolore

und mindestens ein weiteres Zweitrang

mangelnder Compliance oder nich

Bild

Kasuistik - Anhaltender Husten

Röntgen- Thorax mit fortgeschrittener Lungen- Tbc

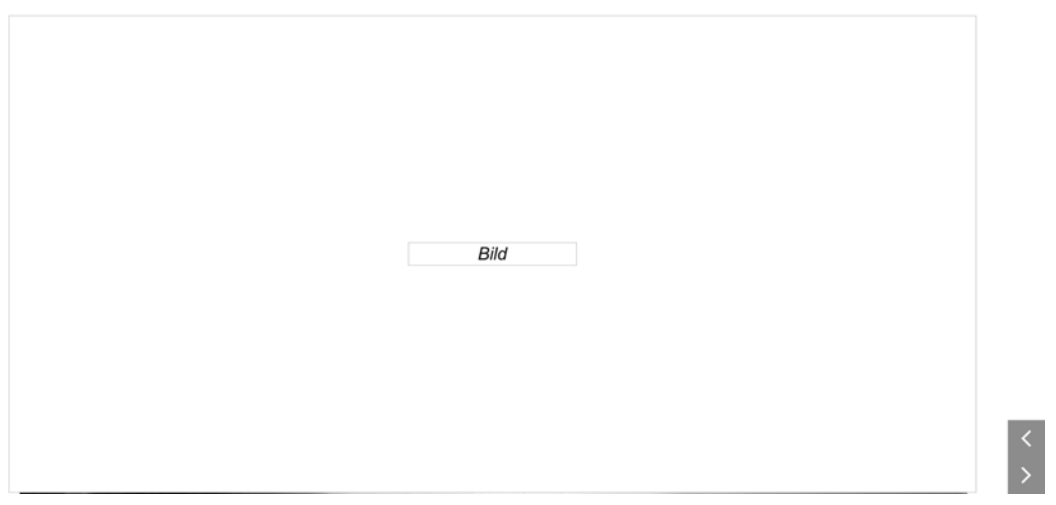




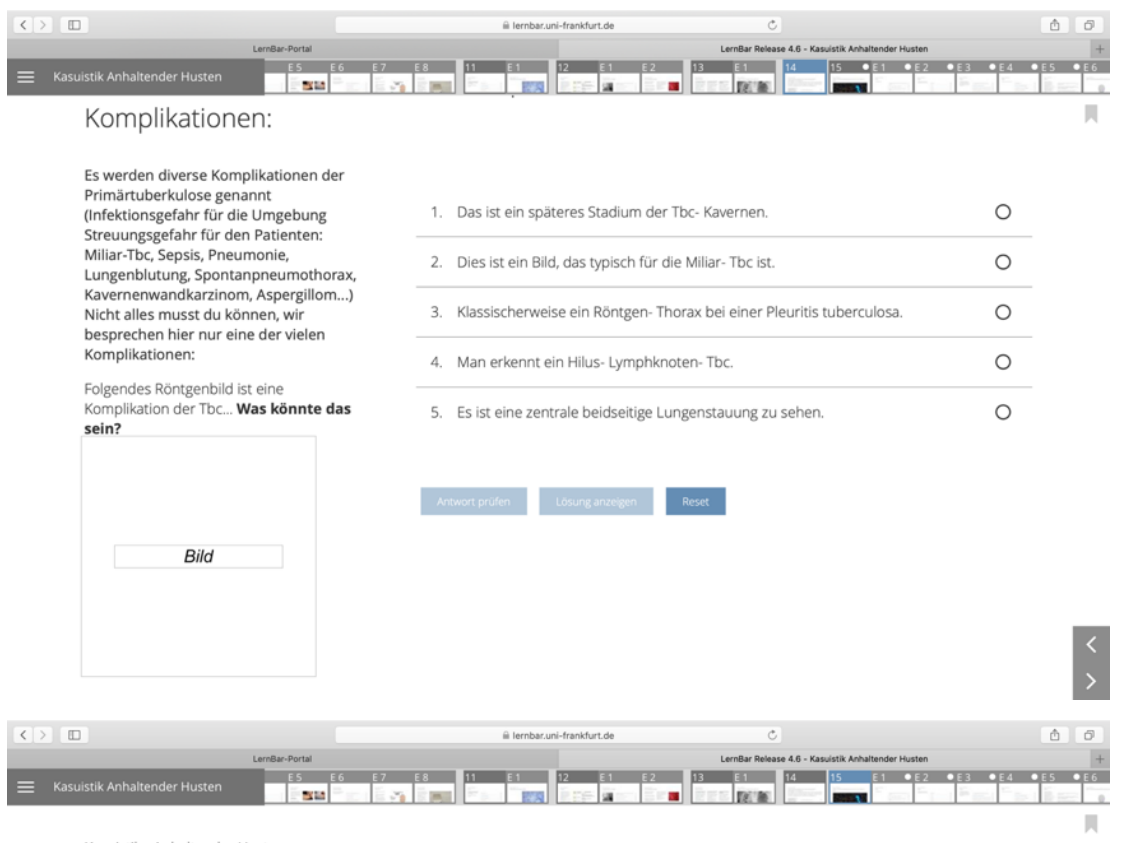

Kasuistik - Anhaltender Husten

Super, nun ist die Kasuistik abgeschlossen. Zum Schluss noch 5 Fragen zum üben :)

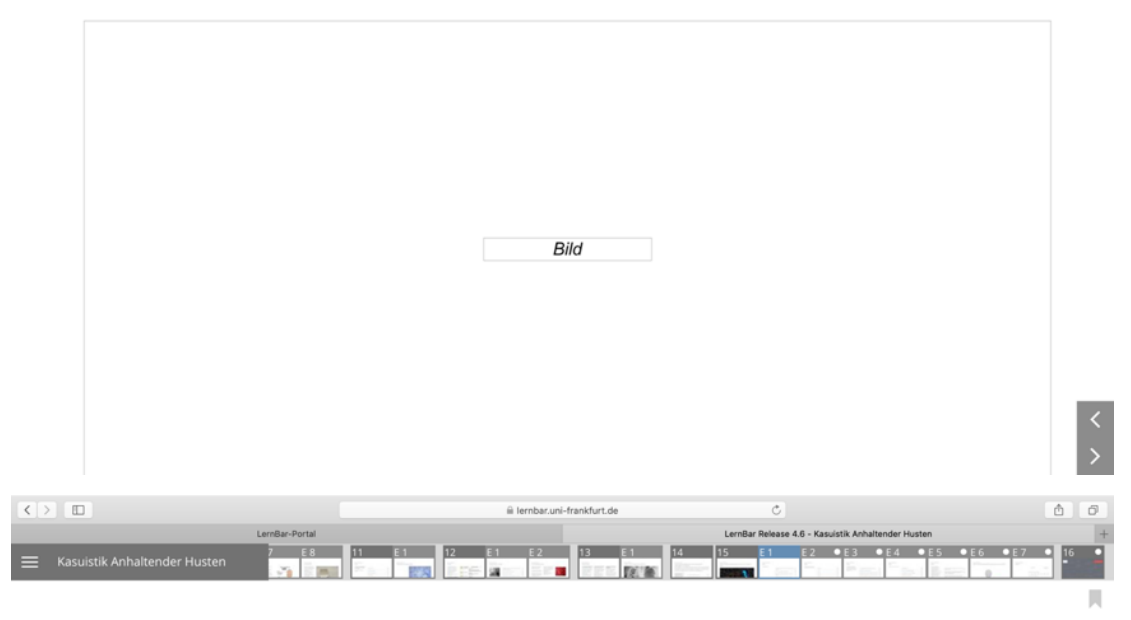

Frage 1

Welche Aussage trifft zu?

\begin{tabular}{l} 
1. Der THT erlaubt die Unterscheidung zwischen latenter Infektion und aktiver \\
Erkrankung. \\
\hline 2. Der THT ist als Routinetest bei Gesunden geeignet. \\
\hline 3. Die BCG-Impfung wird heutzutage noch eingeschränkt von der STIKO empfohlen. 0 \\
\hline \begin{tabular}{l} 
4. Bei im Ausland erworbener Tuberkulose besteht keine namentilche Meldepflicht. \\
\hline 5. Bei HIV- positiven Patienten kommt es beim THT zu falsch-negativen \\
Ergebnissen.
\end{tabular} \\
\hline
\end{tabular}




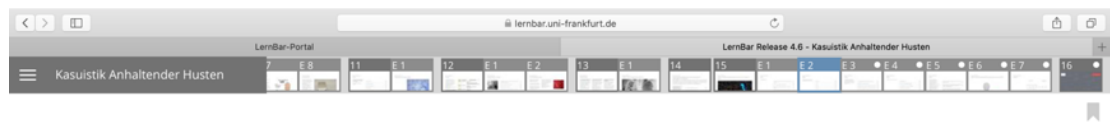

Kasuistik - Anhaltender Husten

Frage 2

Welches Antibiotikum gehört nicht zum

Standardtherapieschema bei Tuberkulose?

\begin{tabular}{lc} 
1. Moxifloxacin & 0 \\
\hline 2. Isoniazid & 0 \\
\hline 3. Rifampicin & 0 \\
\hline 4. Pyrazinamid & 0 \\
\hline 5. Ethambutol & 0
\end{tabular}

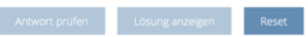

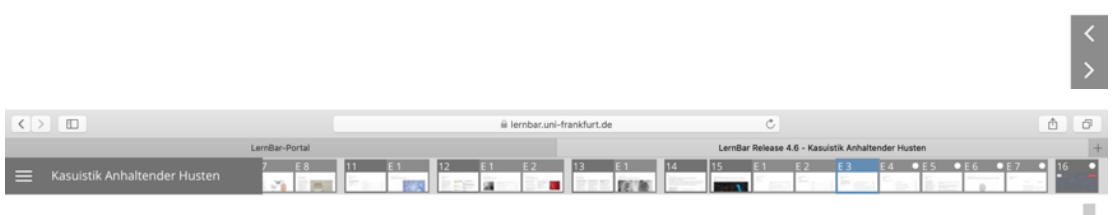

Kasuistik - Anhaltender Husten

Frage 3

Welche der genannten Maßnahmen komm

Krankeghausstation bel inem Parienter

eine offene Lungentuberkulose

\begin{tabular}{ll}
\hline 1. Isolierung des Patienten durch Unterbringung im Einzelzimmer & O \\
\hline 2. Meldung an das zuständige Gesundheitsamt & 0 \\
\hline 3. spezielle Entsorgung der mit infektiösem Material kontaminierten Abfallle & 0 \\
\hline 4. umgehende BCG-Impfung bei den Tuberkulin- positiven Kontaktpersonen & 0 \\
\hline $\begin{array}{l}\text { 5. Röntgenuntersuchung des Thorax bei Kontaktpersonen, bei denen im aktuellen } \\
\text { zeitlichen Zusammenhangs eine Tuberkulinkonversion stattgefunden hat. }\end{array}$ & 0
\end{tabular}

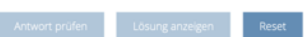

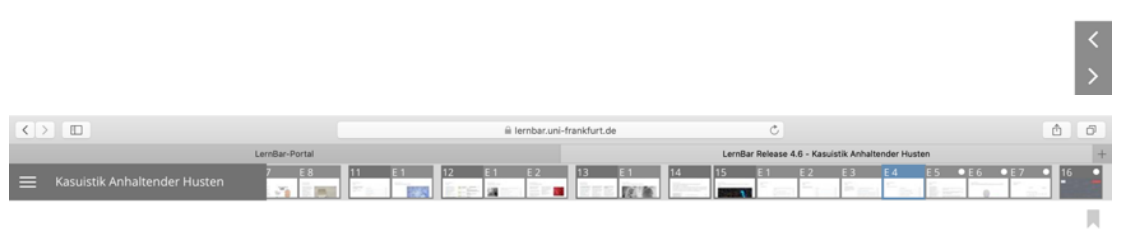

Kasuistik - Anhaltender Huster

Frage 4

Was trifft nicht zur Miliar- Tuberkulose zu?

\begin{tabular}{lc} 
1. Befällt häuffig die Lunge. & O \\
\hline 2. Nicht selten kommt es zur Hepatosplenomegalie. & 0 \\
\hline 3. In der Regel kommt es zu einem isolierten Organbefall. & 0 \\
\hline 4. Nebennieren können auch betroffen sein. & 0 \\
\hline 5. Es gibt auch eine meningeale Form der Miliar- Tuberkulose. & 0 \\
&
\end{tabular}




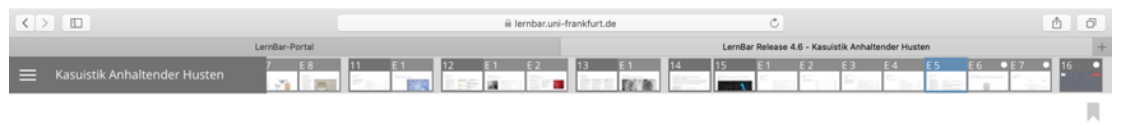

Kasuistik- Anhaltender Husten

Frage 5

Während eines europäischen

erin in der

Notfallaufnahme eines grö̉ere

notfallmäßigen Aufnahmeuntersuchung

eines stark betrunkenen

Festivaltellinehmers aus Osteuropa hustet

dieser die PJlerin mehrmals an. Am

Folgetag efratrin diese, dass bei inm der

trifft am

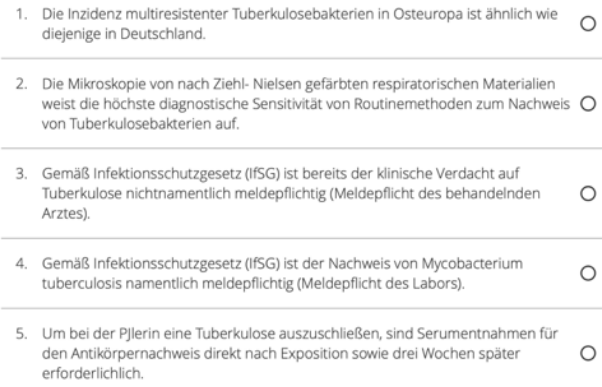

2. Die Mikroskopie von nach Ziehl- Nielsen gefärbten respiratorischen Materialien weist die höchste diagnostische Sensitivität von Routinemethoden zum Nachweis von Tuberkulosebakterien auf. 3. GemäB Infektionsschutzzesetz (IfSG) ist bereits der klinische Verdacht auf
Tuberkulose nichtnamentlich meldepflichtig (Meldepflicht des behandelnden Arztes)

4. Gemäß Infektionsschutzgesetzz (IISG) ist der Nachweis von Mycobacterium

5. Um bei der PJlerin eine Tuberkulose auszuschließen, sind Serumentnahmen für erforderlichlich

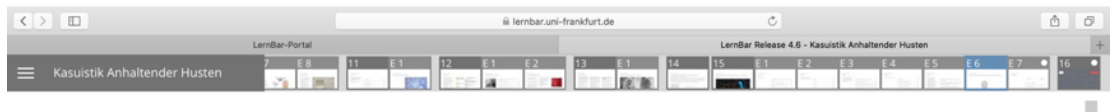

Kasuistik - Anhaltender Husten

Super! Du hast die Kasuistik geschafft! Bitte evaluiere noch kurz die Kasuistik!

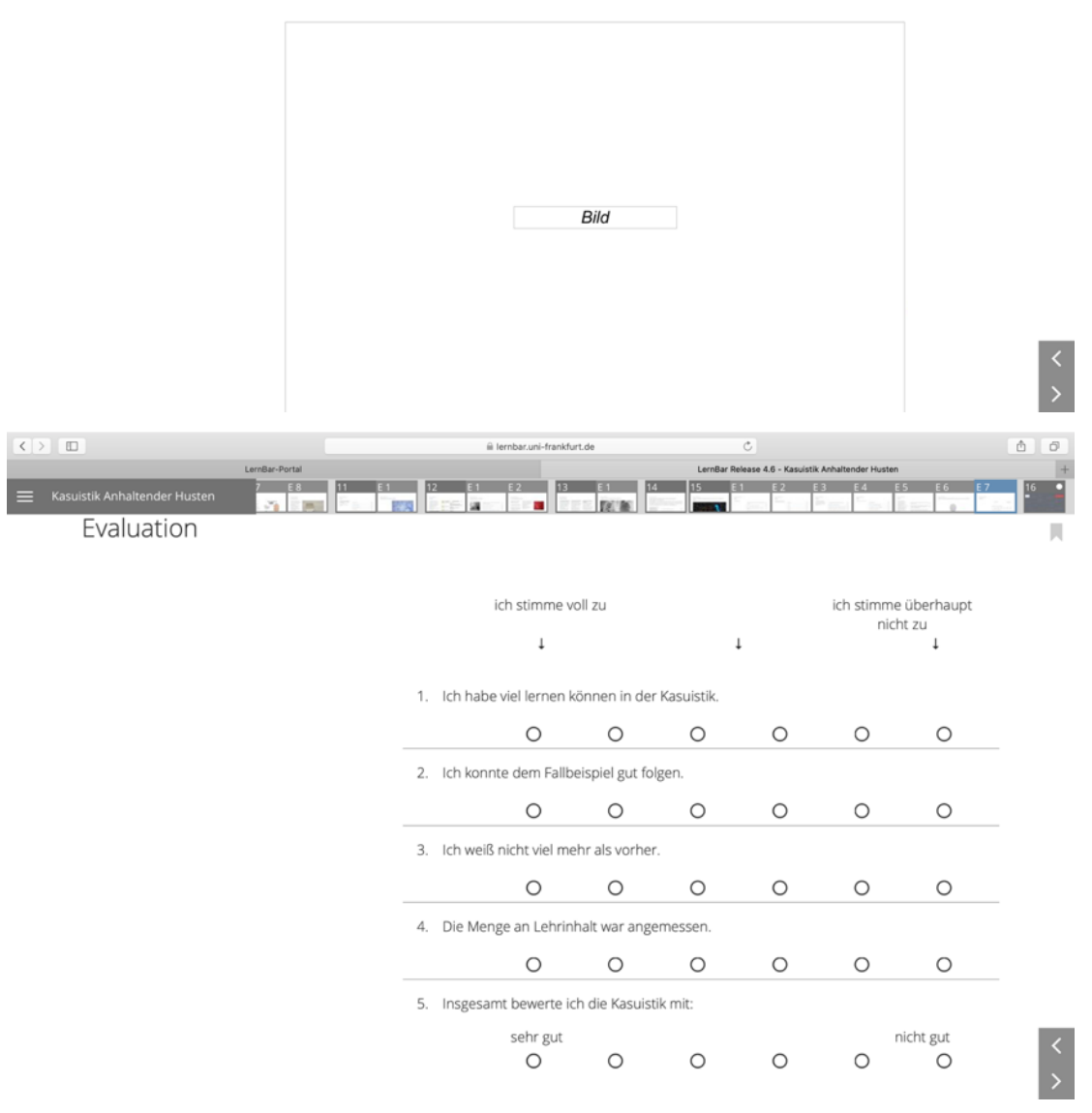




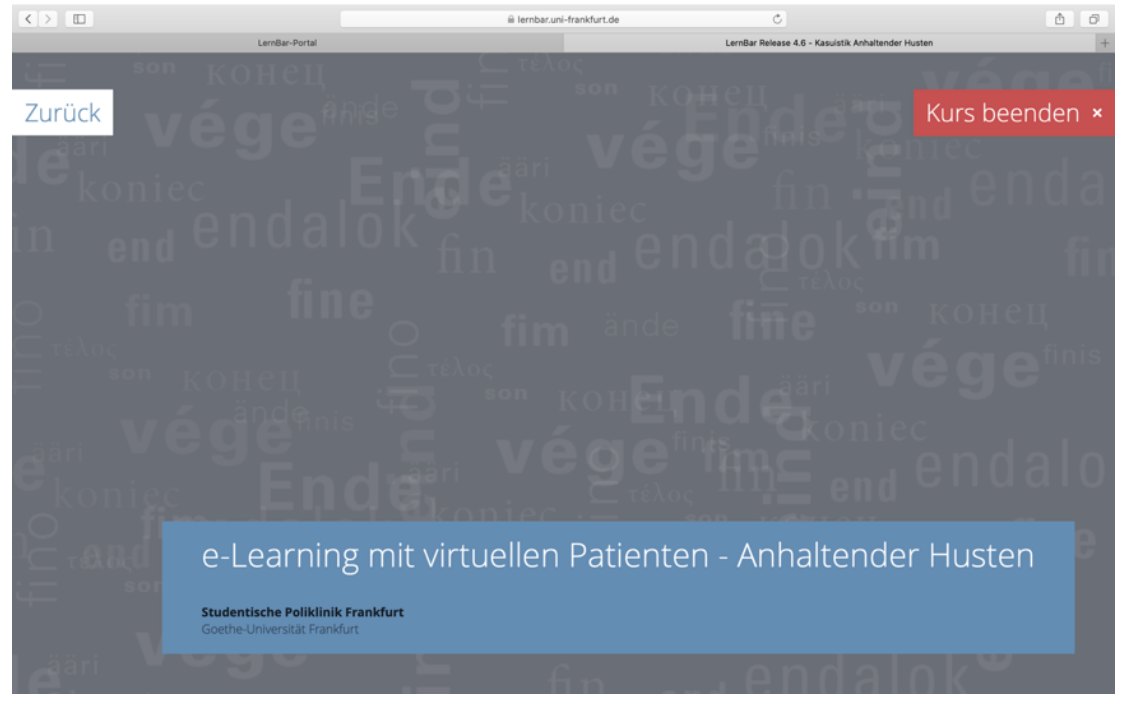


Kasuistik Bauchschmerz

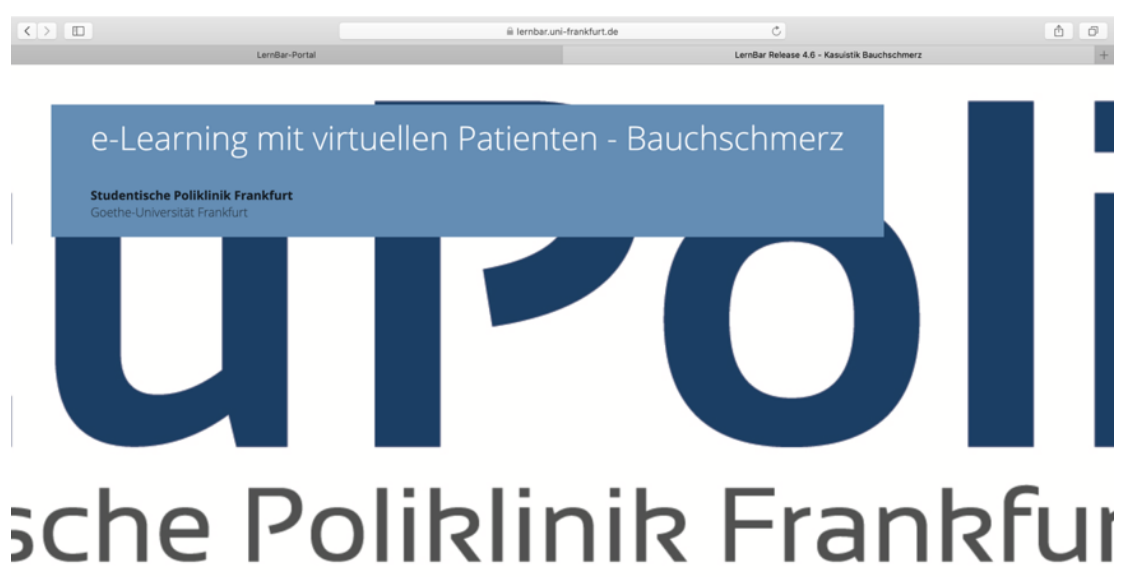

Goethe Universität KURS STARTEN

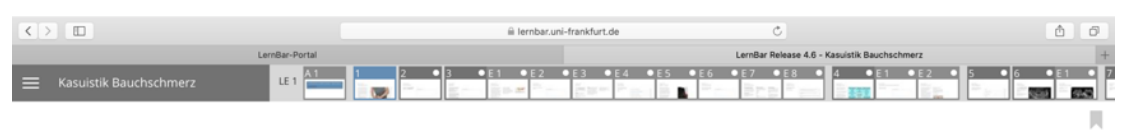

Kasuistik Bauchschmerz

Thema: Bauchschmerz

Hallo und willkommen zur Kasuistik

Bauchschmerz! Hinter Bauchschmerzen

stehen, wichtig ist hier natürlich (wie

immer) eine sehr genaue Anamnese un

die Ursache etwas einzugrenzen. Ist der

Bauchschmenz akut oder chronisch? Wo

und und.

th dieser Kasuistik wirst du ternen wekhe

Patentenaussagen dir bestimmte Hinweise

geben können und was für diagnostische

Mittel dir zur Verfugung stehen.

unserem Fallbeispiel! Vorher gib bitte noch

deinen Namen und deine Matirkelnummer

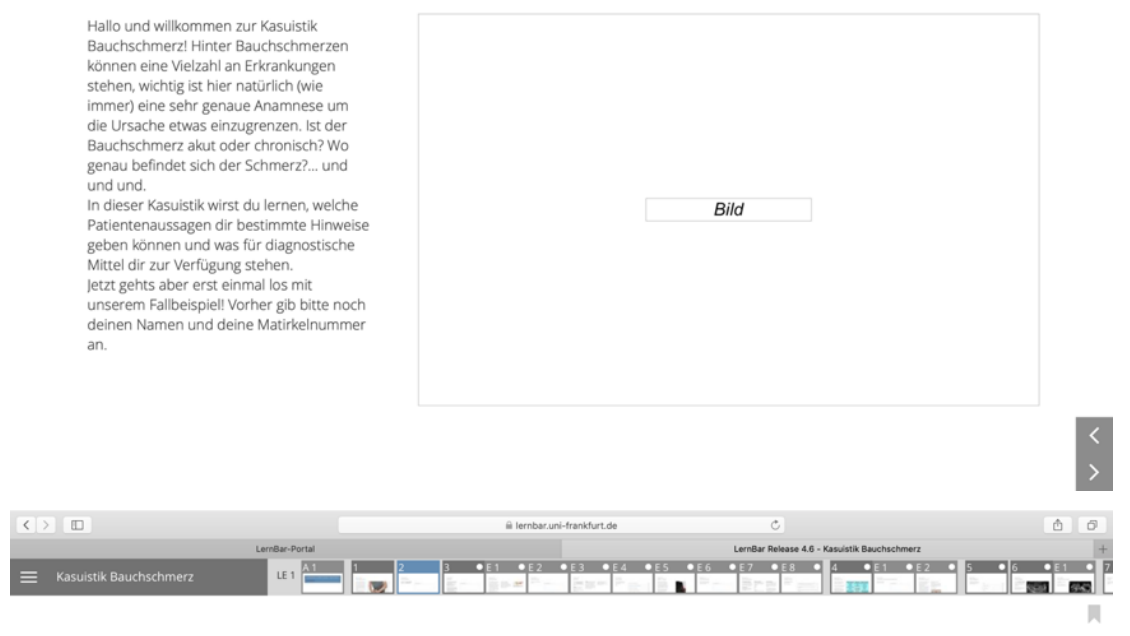

Kasuistik Bauchschmerz

Persönliche Daten

Bitte schreibe in das Feld deinen Vor-und

Namen und deine Matrikelnummer. 


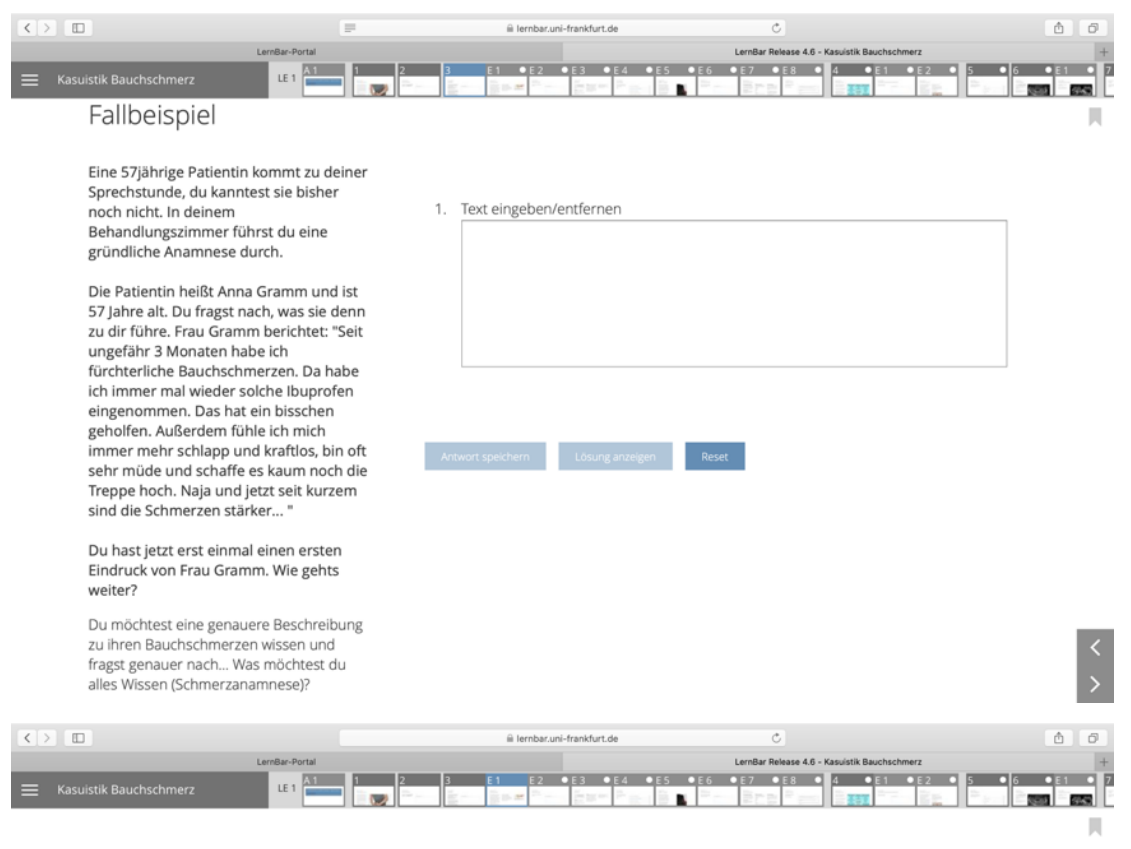

Kasuistik Bauchschmerz

Schmerzanamnese

Der Schmerz besteht seit 3 Monaten,

wobei er eher zugenommen hat und set

verschlechterung zu beobande ist.

keine Kausailtăt bekannt. Der Schmerz ist

im Oberbauch lokalisiert, jedoch nicht noch

genauer lokalisierbar. Es ist keine

Ausstrahlung zu beobachten. Auf einer

Skala von 1 - 10 nennt Frau Gramm die 4 as

Schmerzintensitat,

Der Schmerz wird als brennend und dump

besser oder schlechter wird, erzählt die

Patientin, dass es nach dem Essen besser

werde, davor eher schlechter sei:

Damit sind alle 7 Attribute zur

Schmerzanamnese abgehakt:

Schmerzlokalisation, Ausstrahlung

der Schmerzen, zeitlicher Zusammenhar

Faktoren der Verschlimmerung'
Erleichterung, Kausalität)

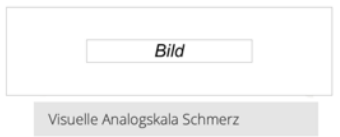

Visuelle Analogskala Schmerz

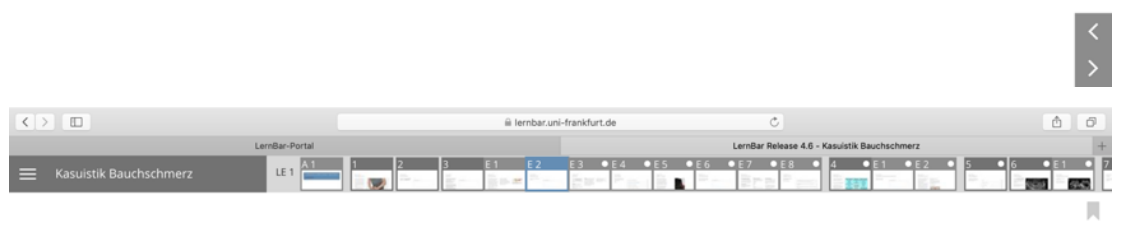

Kasuistik Bauchschmerz

Weitere Anamnese

Nenne nun weittere wichtige Anteile der
Anamnese.

1. Text eingeben/entfernen 


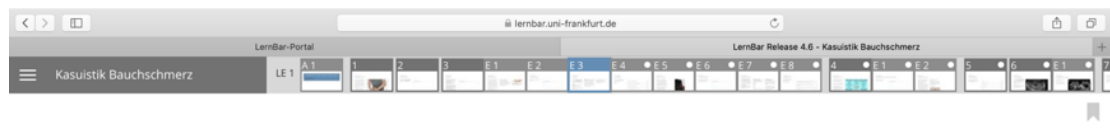

Kasuistik Bauchschmerz

Anamnese

Beim Leitsymptom Bauchschmerz solltest

duimmer die vegetalive Anamnese

oben nach unten" durch die Organsysteme

arbeiten

Abdomen: Hier effragst du alles was den

or $\operatorname{trin}$ ? Sind Schmerzen oder andere

Beschwerden beim Toilettengang

abfragen (NachtschweiB, Fieber,

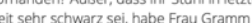

fall nicht vorhanden ist.

Kopf/Hals: Schwindel, Kopfschmerzen

keine anter

rungen werden vernein

ubel sei ihr ab und zu schon.

Thorax: es bestehen keine Atemnot oder

Druck auf der Brust

\section{,}




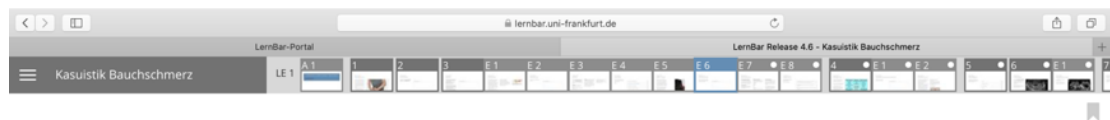

Kasuistik Bauchschmerz

noch mehr Anamnese

Was möchtest du in deiner Anamnese noch

alles wissen? Zähle alle Punkte auf, die du

Frau Gramm fragst.
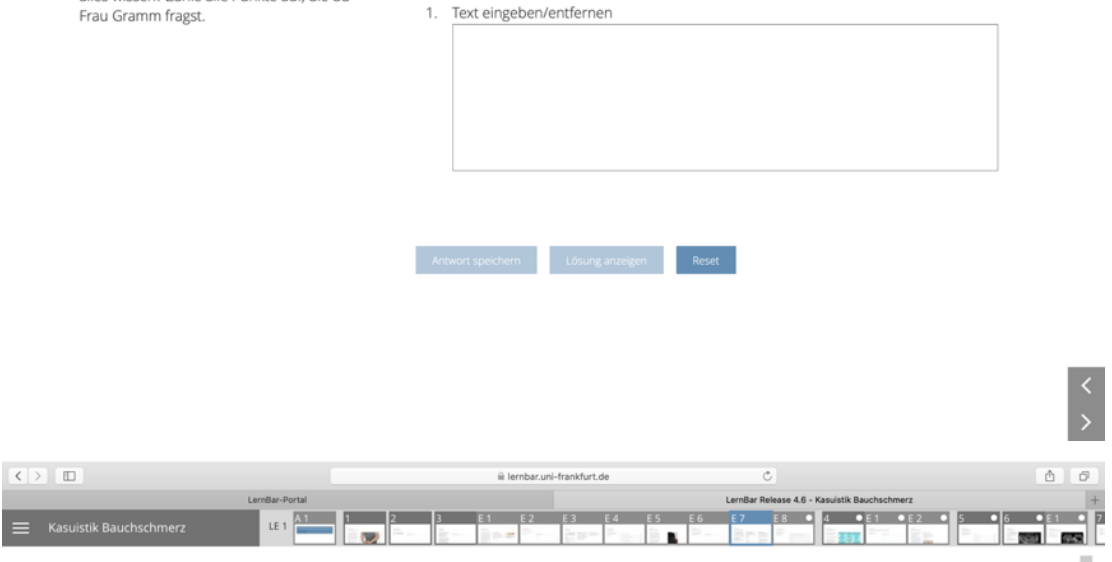

Kasuistik Bauchschmerz

Das kommt dabei raus

Vorerkrankungen/ Operationen:

Fru Gramm leidet an Diabetes mellitus Ty

2, ist Hypentonimetin und habe auch

Thymektomie 1993 keine Operationen.

Familienanamnese:

Der Vater starb im Alter von 67 Jahren a

einem Atersheim.
Medikamentenanamnese

Dust Glück und Frau Gramm hat ihren Du hast Gluck und Frau Gramm hat inren

Metformin

- Thyrox

Enalapr

HCT

ASS

Clopidogrel
Genussmittelanamnese

wruu Gramm trinkt "gelegentich ein Glas

Außerdem raucht sie seit 25 tahren eine Packung Zigaretten täglich. Drogen nehme sie keine.

Zur Vollståndigkeit erhebst du noch die Sozilalanamnese, in der du ertahrst, dass

und 3 Kinder hat (alle wurden per und 3 Kinder hat (ante wurden $p$

\section{,}




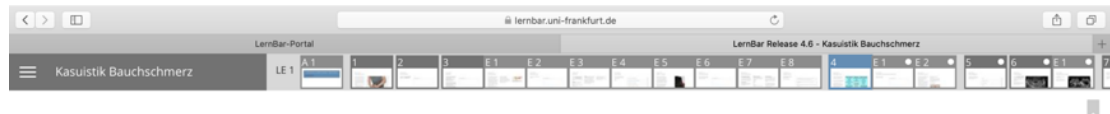

Kasuistik Bauchschmer

Weiter geht es mit...

Korrekt, der nächste Schritt ist die

die abdominelle Untersuchung.

Hier ist ein Video (nww) zur abdomineller

enau an.

Bei der Inspektion (hier vorweg

Narben, Hernien, Venenzeichnung und

Behaarungstyp (Bauchglatze z.B.). Frau

Gramm ist in einem guter

Allgemeinzustand (AZ) und

Ernāhrungszustand (EZ). AuBer einer

keine pathologischen Befunde 74

erkennen

c

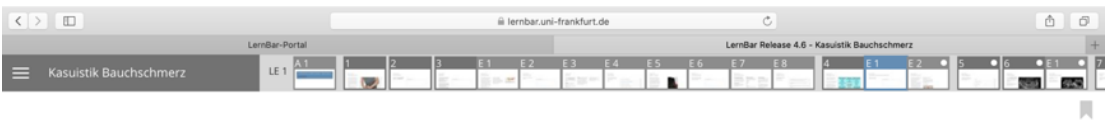

Kasuistik Bauchschmerz

Normalbefund der abdominellen Untersuchung

Nenne den Normalbefund der

Untersuchung:

1. Text eingeben/entfernen
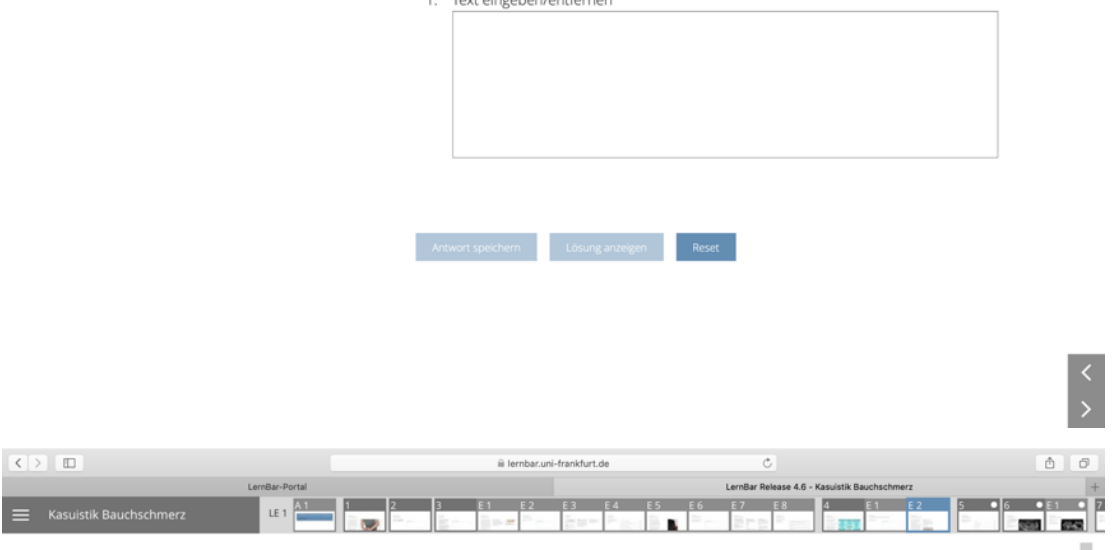

Kasuistik Bauchschme

Befund bei Frau Gramm

Du hörst regelrechte Darmgeräusche (DG) über allen 4 Quadranten und einen

tympanischen Klopischall uber alien 4

Normalbefund der Fall.

Untersuchung):

keine Resistenzen oder Damforderungen

autasten dephnherenus ist normal.

deutichen Druckschmerz mit zusatzlich

Walzen oder Resstenzen ts stbar, Leber

und Milz sind ohne pathologischen Befund

Das Nierenlager ist tastbar, jedoch kein

Klopfschmerz vorhanden. Zudem sind kein

Hernien tastbar oder durch Husten

Abdominelle Regionen:

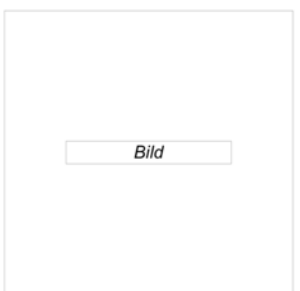




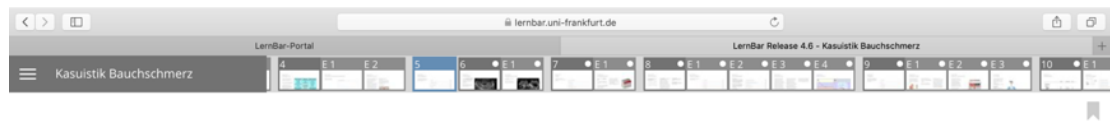

Kasuiststik Bauchschmerz

Differentialdiagnosen

Mit Hintergrund der Anamnese und

(d)

dir Differentialdiagnosen...

\begin{tabular}{lc} 
1. Anämie & $\square$ \\
\hline 2. Ulcus ventricull/ duodeni & $\square$ \\
\hline 3. untere Gl- Blutung & $\square$ \\
\hline 4. Herzinfarkt & $\square$ \\
\hline 5. akute oder chronische Gastritis & $\square$ \\
\hline 6. Cholezystitis & $\square$
\end{tabular}

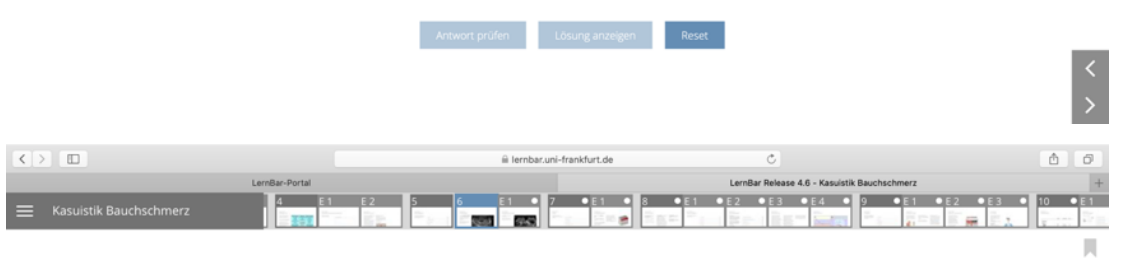

Kasuistik Bauchschmerz

Verdachtsdiagnose

Du hast den Verdacht auf eine Gastritis

und wahlst die Sonographie um noch mal

mit diesem diagnostischen Mitte!

bestâtigen lässt.

Schaue auch in diesem Video ( $\mathrm{nmw}$ ) dir

gerne die Sonographie des Abdomens an,
wenn du interessiert bist.

Bild

Normale Sonographie des Abdomens

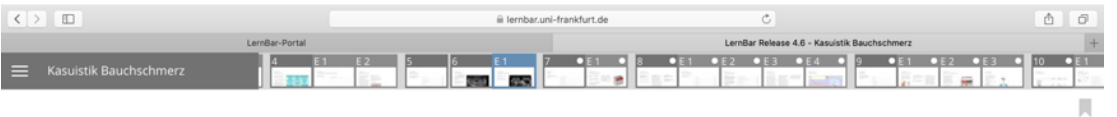

Kasuistik Bauchschmerz

Sonographie von Frau Gramm

Frau Gramm hat keinen normalen

Sonograpie-Befund

Man erkennt eine angeschwollene

Magenschleimhaut und Ulzerationen.

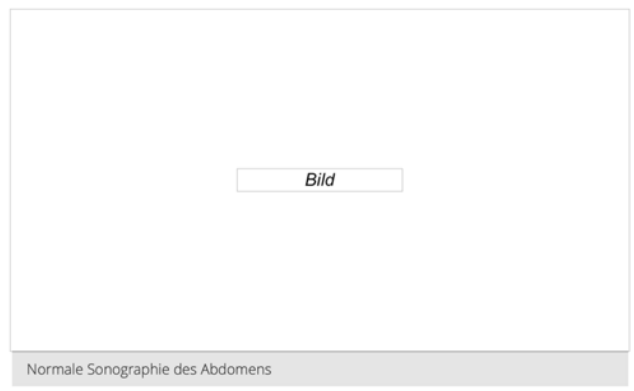




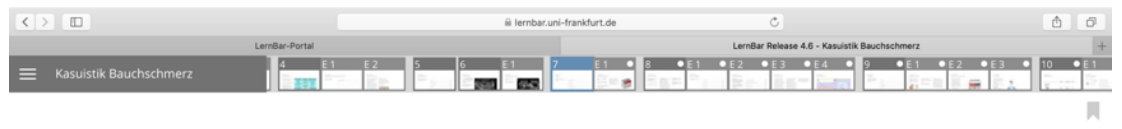

Kasuistik Bauchschmerz

Therapie

Was könntest du Frau Gramm

thre Beschwerden

verbessern?

\begin{tabular}{lc} 
1. Clarythromycin $500 \mathrm{mg}$ & 0 \\
\hline 2. Iberogast Tropfen & 0 \\
\hline 3. Omeprazol $40 \mathrm{mg}$ & 0 \\
\hline 4. Arzneitee (Magen- Darm- Tee) & 0 \\
\hline 5. Pantoprazol $500 \mathrm{mg}$ & 0
\end{tabular}

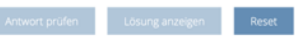

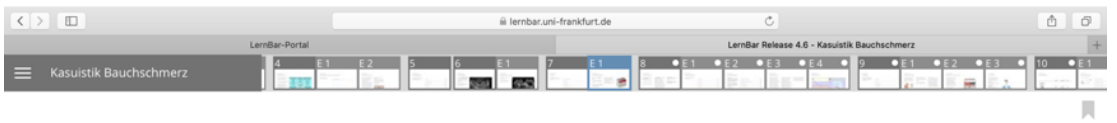

Kasuistik Bauchschmerz

Kurzfristige Behandlung ...

Die korrekte Wahl wäre: Omeprazol 40mg

Du kannst natürlich auch Pantoprazol

verschreiber jedoch ist die Dosierur viel

zu hoch!

a du aber nicht nur symptomatisch

möchtes

Vorher aber denks du noch mal gründlich

Clarythromycin kommt bei der Behandlung

einer Gastritis auf jeden Fall in Frage, hilft

jecractich sorore

Der Magen- Darm- Tee und Iberogast

(pflanzliches Heilmittel) sind eher

sekundäre Wahl, da die Schmerzen schnel

und gezielt mit einem Protoneninhibitor

uber die chronische Gastritis nach...

Bild
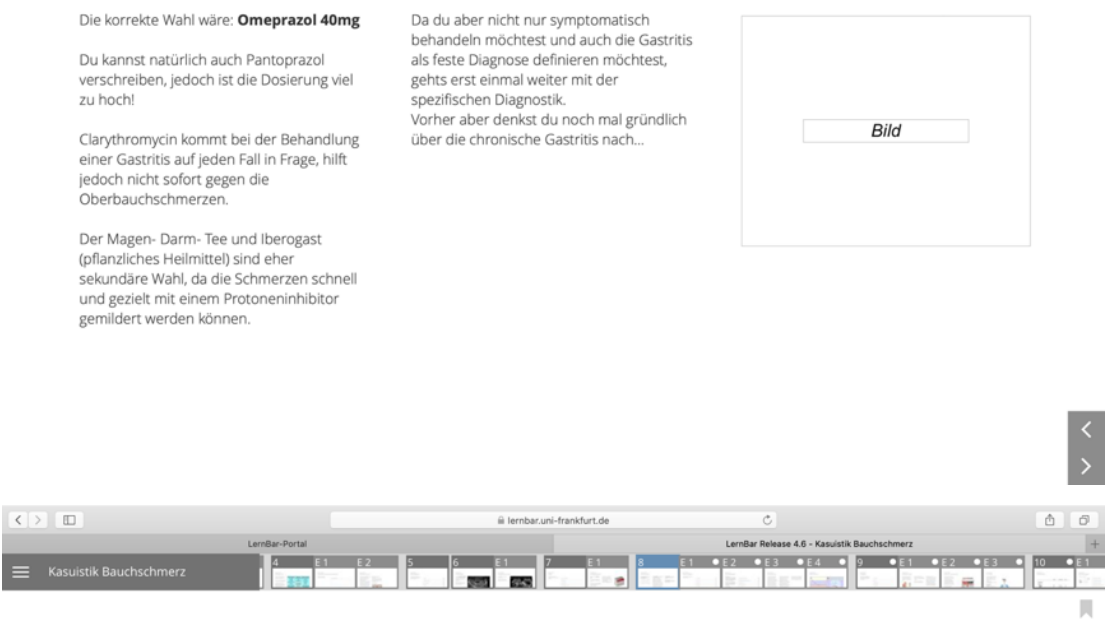

Kasuistik Bauchschmerz

Chronische Gastritis Die chronische Gastritis wird durch die
ABC- Klassifikation eingeteilt:

Typ A- Gastritis = Autoimmungastritis

Typ B- Gastritis = Bakterielle Gastritis

Typ- C- Gastritis = Chemische Gastritis
Die Typ- A- Gastritis hat eine unbekannte Atiologie, fest steht jedoch, dass Matoanthorper die Belegzetien im Belegzellen sind bekannterweise verantwortlich für die Produktion der Magensäure und des Intrinsic Factors. Im weiteren Verlauf kann es zum Vitamin- $B$ 12-Manget kommen, Eine Typ-A-Gastris ist daner haufig mit ener perniziosen

ist im Prinzziper eine (ene pernizose Anamie Intrinsic Factor-Mangels bei TypA Gastritis.
Die Typ- B- Gastritis wird meist durch eine

Die Typ-c-Gastritis wird durch direkte Nikotin u.a. verursacht. 


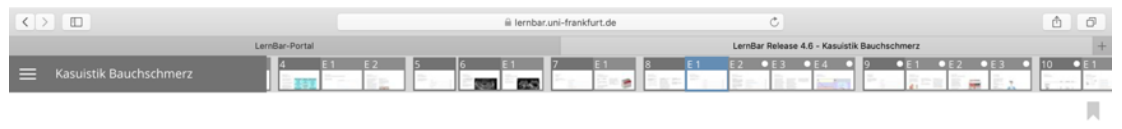

Kasuistik Bauchschmerz

Was ist am häufigsten?

Was denkst du ist die häufigste Form der chronischen Gastritis?

\begin{tabular}{lc} 
1. Typ A mit 50\% & 0 \\
\hline 2. Typ B mit 50\% & 0 \\
\hline 3. Typ C mit 55\% & 0 \\
\hline 4. Typ B mit 80\% & 0 \\
\hline 5. Typ A mit $80 \%$ & 0
\end{tabular}

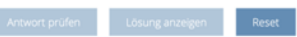

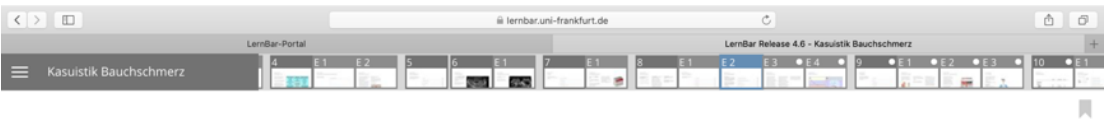

Kasuistik Bauchschmerz

Neue Erweiterungsseite

Zur Frage vorher:

Deastritis macht mit ca. $80 \%$ den

Hauptanteil aus, die Typ C Gastritis ist zu
ca. $15 \%$ vertreten und ganz am Ende gibt

es noch zu 5\% die Typ A Gastritis.

Das vorkommen von HP. Besiedlungen

korreliert in etwa mit dem Alter, im Alter

Welches der folgenden

Antwortmöglichkeiten trägt NICHT

unmittelbar zum Pathomechanismus einer

\begin{tabular}{lc} 
1. erhöhte Säurebildung im Magen & $\square$ \\
\hline 2. deszendierene Ausbreitung der Entzündung & $\square$ \\
\hline 3. Ureasebildung durch HP & $\square$ \\
\hline 4. Ammoniakschutzfilm des HP & $\square$ \\
\hline 5. Hypochlorhydrie & $\square$
\end{tabular}

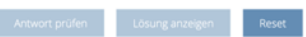

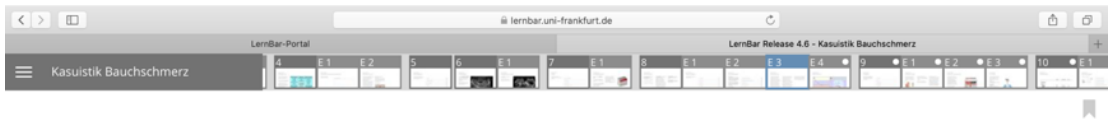

Kasuistik Bauchschmerz

Pathomechanismus

Zunächst einmal wird die bakterielle

Gastritis hauptsachilch durch eine Infektion

pylori (HP) verursacht. Helicobacter pylo

hat die Eigenschaft eine Urease zu

produzieren, welche durch Spaltung vo

Harnstoff Ammoniak entstehen lässt.

Dadurch wird eine Art Schutzfilm für das

HP gebilidet, da das Ammoniak den $\mathrm{pH}$ -

Wertum das bakentum herm ethont. So

Magerleben dien dieu.

Im weiteren Verlauf werden im Magen

meniger Muzine produziert, dafür aber

mentsane un es komint zur entzandun

aszendierender Ausbreitung Es kommt 7

Verschiebung der Antrum-Korpus- Grenze

nach oben und die Zahl der Belegzellen

nimmt ab. Durch Atrophie der

Drusenkörper/ Belegzellen kommt es zur

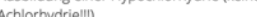

Achlorhydrie!l!)

Dafigsten im Antrum lostelisitis auch an
Die gesuchte Antwort zur vorherigen Frage

ist also die Falschaussage "deszendierende

nämlich eine aszendierende Ausbretist 


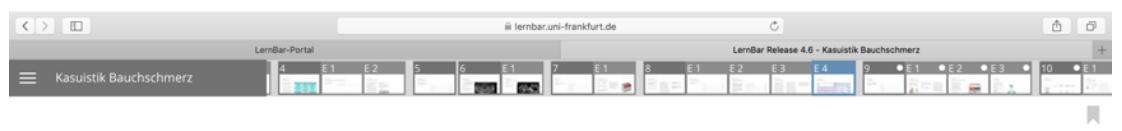

Kasuistik Bauchschmerz

Pathomechanismus

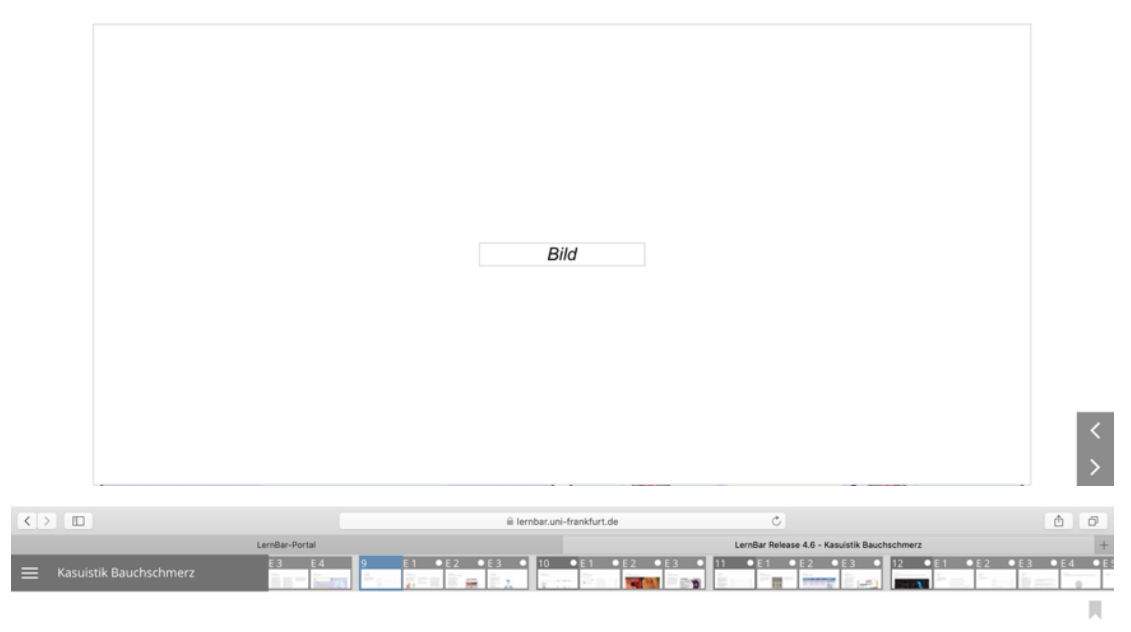

Kasuistik Bauchschmerz

HP-Diagnostik

Welches der folgenden diagnostischen

Unerscheiduch am wenigsten fur die

B-Gastritis?

\begin{tabular}{lc} 
1. C13 Atemtest & $\square$ \\
\hline 2. Guajak- Test & $\square$ \\
\hline 3. Urease Schnelltest & $\square$ \\
\hline 4. D-Xylolose- Test & $\square$ \\
\hline 5. Blutbild & $\square$
\end{tabular}

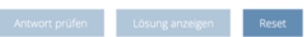

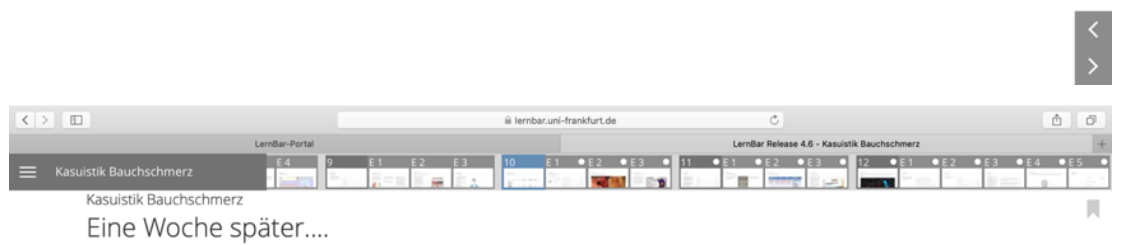

Eine Woche später.

\begin{tabular}{|c|c|c|c|c|}
\hline & & Resultat & Referenzbereich & Einheit \\
\hline Hämatologie & & & & \\
\hline $\begin{array}{l}\text { BLUTBILD } \\
\text { Erythrozyten } \\
\text { Hämoglobin } \\
\text { Hämatokrit } \\
\text { Mittleres Zellvolumen (MCV) } \\
\text { MMttleres Zelliămoglobin (MCH) } \\
\text { Mittlere korpuskuläre Hämoglobin } \\
\text { Konzentration (MCHC) } \\
\text { Erythrozyten Verteilungsbreite } \\
\text { Thromboyyten } \\
\text { Mittleres Thrombozytenvolumen } \\
\text { Leukozyten }\end{array}$ & $\begin{array}{l}\downarrow \\
\vdots \\
\vdots \\
\vdots\end{array}$ & $\begin{array}{c}3.4 \\
6,7 \\
32 \\
55.3 \\
16.6 \\
33.3 \\
14.3 \\
306 \\
9.9 \\
18.58\end{array}$ & $\begin{array}{r}4.4=5.8 \\
13.5=18.0 \\
40.0=52.0 \\
78.0=98.0 \\
27.0=33.0 \\
32.0=36.0 \\
11.0=16.0 \\
150=350 \\
7.0=13.0 \\
4.0=10.0\end{array}$ & $\begin{array}{l}\mathrm{Tn} \\
\mathrm{g} / \mathrm{dl} \\
\% \\
\mathrm{fl} \\
\mathrm{pg} \\
\mathrm{g} / \mathrm{dl} \\
\% \\
\mathrm{G} / 1 \\
\mathrm{fl} \\
\mathrm{G} / \mathrm{l}\end{array}$ \\
\hline
\end{tabular}




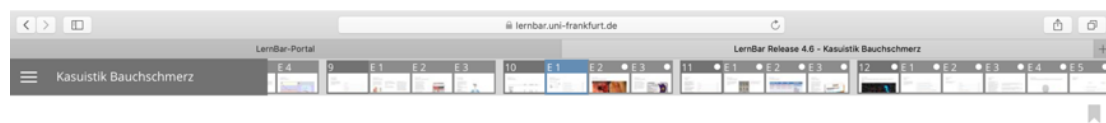

Kasuistik Bauchschmerz

Neue Erweiterungsseite

Was erkennst du im Blutbild?

\begin{tabular}{ll} 
1. mikrozyäre, hypochrome Anämie & 0 \\
\hline 2. normozytäre, normochrome Anämie & 0 \\
\hline 3. makrozyăre, hyperchrome Anămie & 0 \\
\hline 4. Leukozytopenie als Ursache für eine Anămie & 0 \\
5. massive Erythrozytose & 0
\end{tabular}

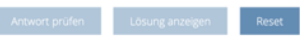

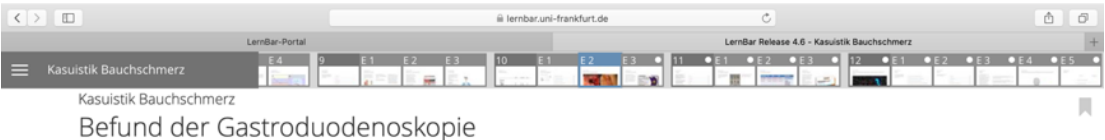

Befund der Gastroduodenoskopie

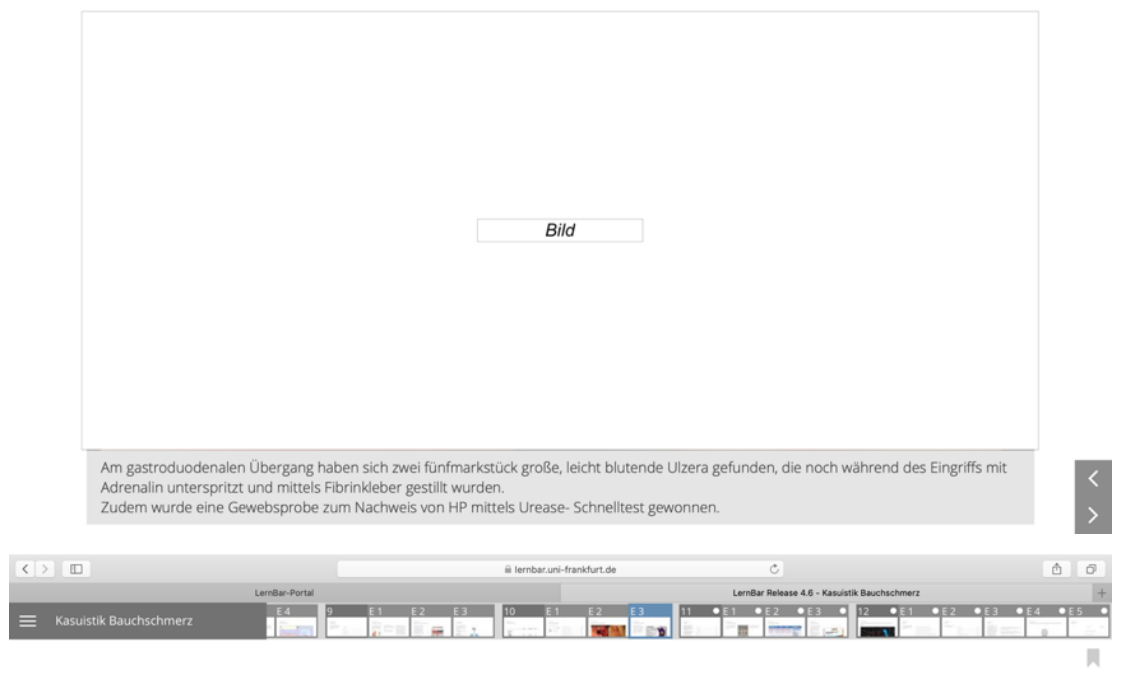

Kasuistik Bauchschmerz

Helicobacter-Urease-Test

Beim Urease-Schnelltest werden

endoskopisch gewonnene Gewebeproben

arease des HP füht über den

Harnstoffs zu einer Freisetzung von

Ammoniak sowie zu einem pH-Anstieg, der

mittels Farbumschlag nachgewiesen und so

einen Hinweis auf eine HP-Infektion liefern 


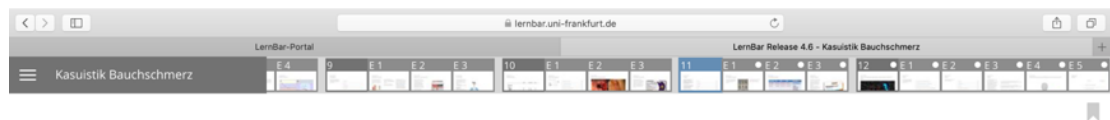

Kasuistik Bauchschmerz

Therapie

Zusammenfassend weiißt du nun: Frau

Gramm hat eine B. Gastritis mit

weißt das Blutbild der Patientin auf eine

Eisenmangelanămie hin.

\begin{tabular}{ll} 
1. Amoxicillin & 0 \\
\hline 2. Clarychromycin & 0 \\
\hline 3. Ciprofloxacin & 0 \\
\hline 4. Bismuth & 0 \\
\hline 5. Metronidazol & 0
\end{tabular}

Du leitest die Eradikationstherapie ein

und vereinbarst mit frau Gramm eine Erfolgskontrolle in 2 Wochen.

Welche der folgenden Medikamente gehört nicht zur Eradikationstherapie bei HP.

5. Metronidazol

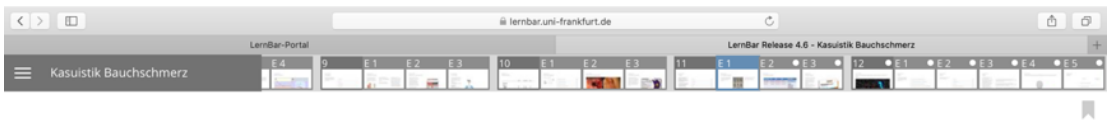

Kasuistik Bauchschmerz

HP-Eradikationstherapie

Erstlinientherapien sind die sogenannten

Triple Therapien. Oft wird Pantoprazol as

verschrieben.

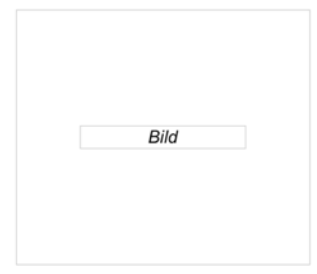

Auch Bismuth kann zum Einsatz kommen:

+ Tetracyclin + Metronidazol

Frech Triple:

Italian Triple:

$\mathrm{PPl}+$ Clarithromycin + Metronidazo

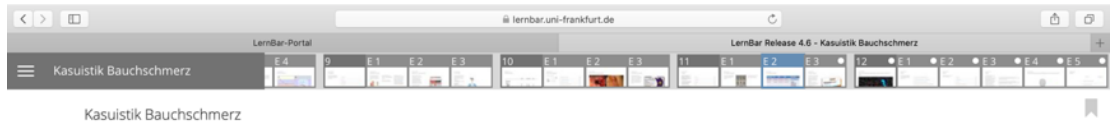

HP-Eradikationstherapie

\begin{tabular}{|c|c|c|c|c|}
\hline Therapieform & PPI & AB1 & AB2 & Bemerkung \\
\hline French Triple & Pantoprazol & Clarithromycin & Amoxicillin & Hohe Eradikationsrate \\
\hline Italian Triple & Pantoprazol & Clarithromycin & Metronidazol & $\begin{array}{l}\text { Geringere } \\
\text { Eradikationsrate, } \\
\text { anwendbar bei } \\
\text { Penicillinallergie }\end{array}$ \\
\hline $\begin{array}{l}\text { Bismuth- } \\
\text { Quadrupeltherapie }\end{array}$ & Pantoprazol & Tetracyclin & Metronidazol & + Bismuth \\
\hline
\end{tabular}

Diese Drei Therapieformen sind die derzeitigen Erstlinientherapien! 


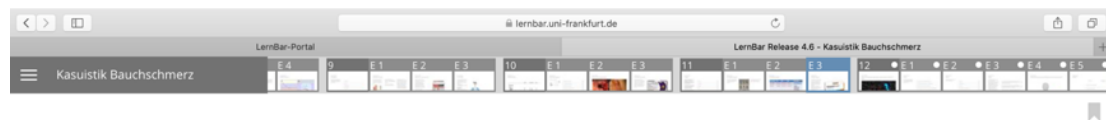

Kasuistik Bauchschmerz

Ende gut, alles gut...?

Nach 10 Tagen kommt Frau Gramm, wie vereinbart, zu dir in die Sprechstunde. De

weitestgehend zurückgegangen und au

der schwarze Stuhl ist kaum noch da.

Außerdem ist sie auch nicht mehr so

abgeschlagen

Oa sezte die Antibiotika ab, aber

zwei Wochen Pantoprazol einzunetmen

bis die Entzündung vollständig

zurückgegangen ist.
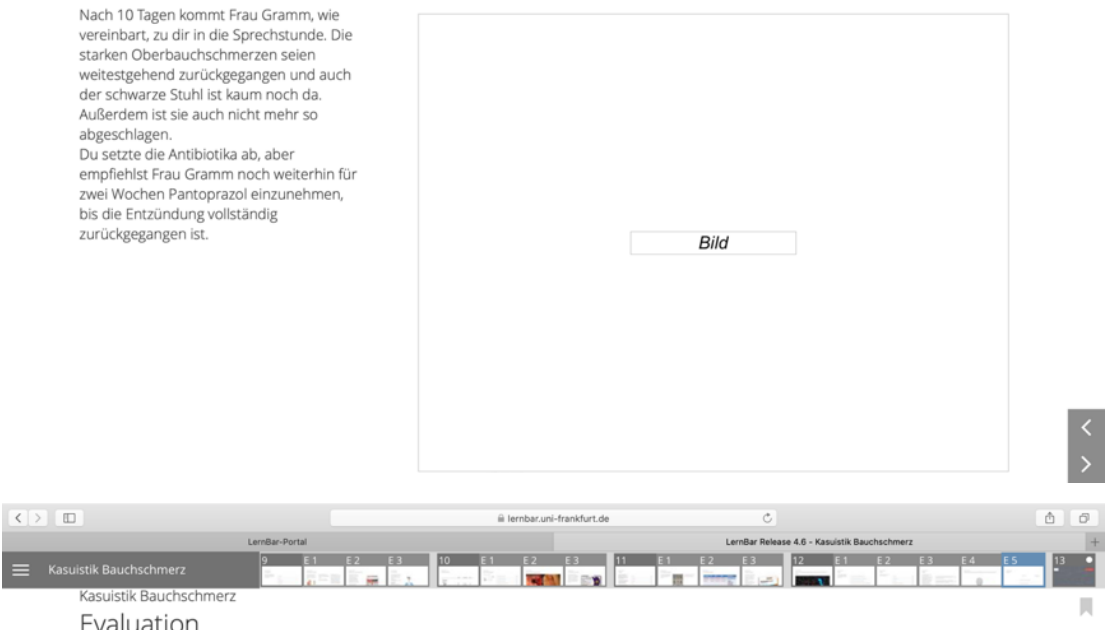

Evaluation
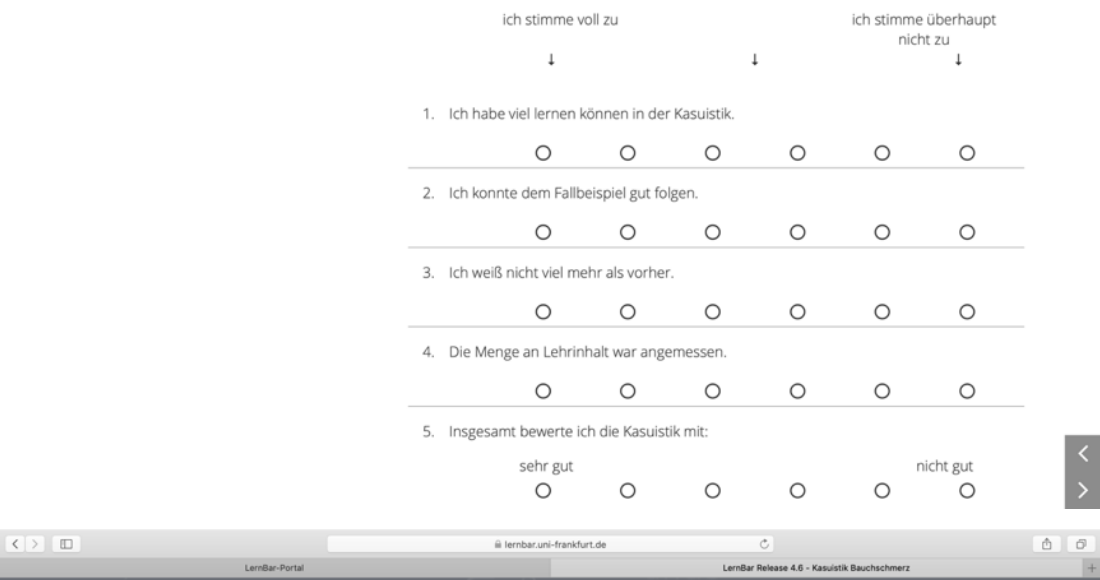

e-Learning mit virtuellen Patienten - Bauchschmerz Studentische Polikkinik Frankfurt 
Kasuistik Dyspnoe und Leistungsminderung

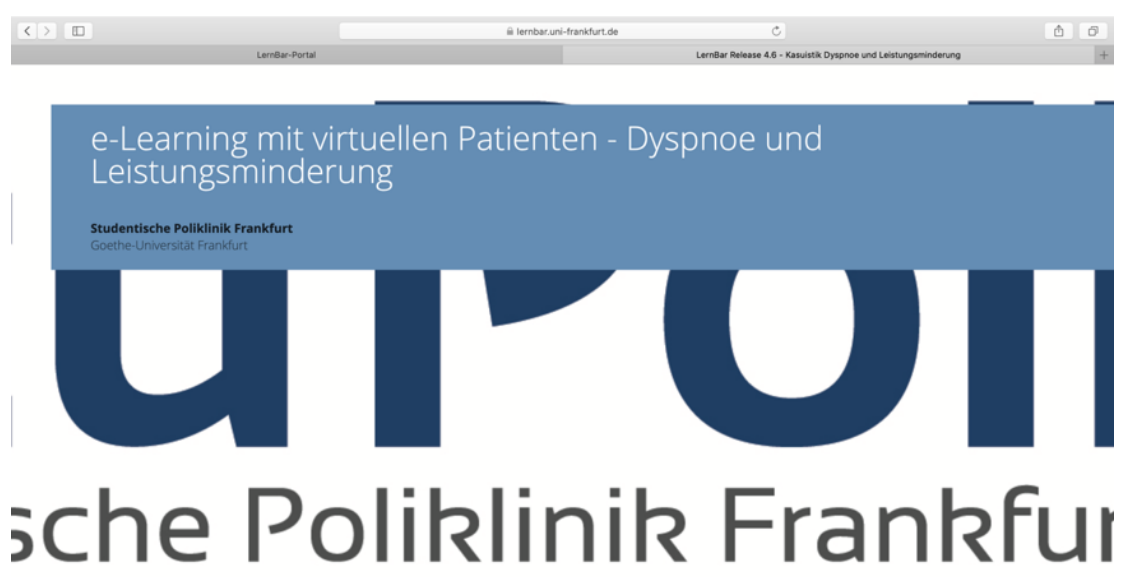

\section{Goethe Universität KURS STARTEN}

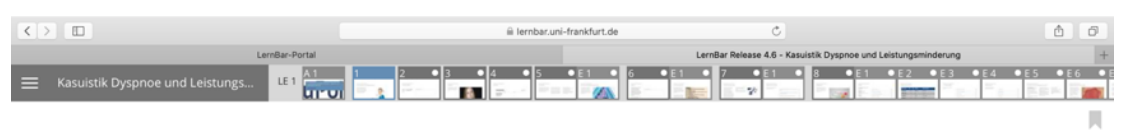

Kasuistik Dyspnoe und Leistungsminderung

Thema: Dyspnoe und Leistungsminderung

Wilkommen zur heutigen Kasuistik über

Dyspnoe und Leistungsminderung.

We

dich zu einer bestim beschaftigen und

durcharbeiten wirst. Viel Spaß!

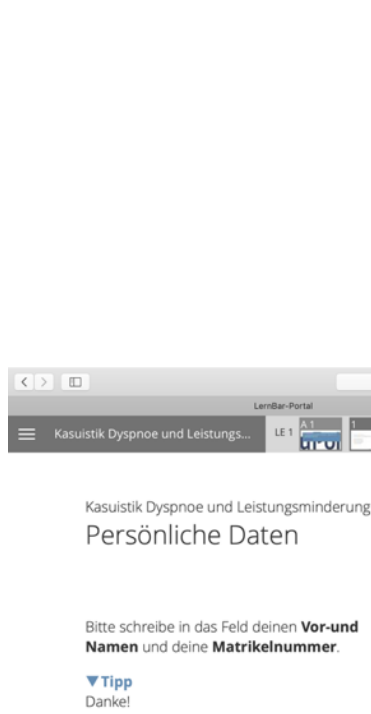

VTipp
Dankel

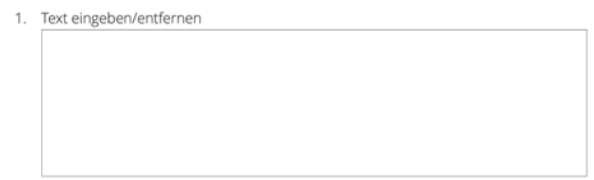




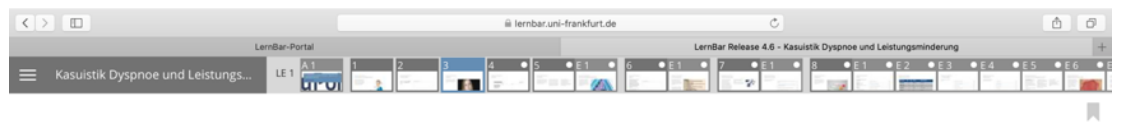

Kasuistik Dyspnoe und Leistungsminderung

Fallbeispiel

Eine junge Dame sucht zum ersten mal

deine Aigemeinarzpraxis in Frankfurt aut

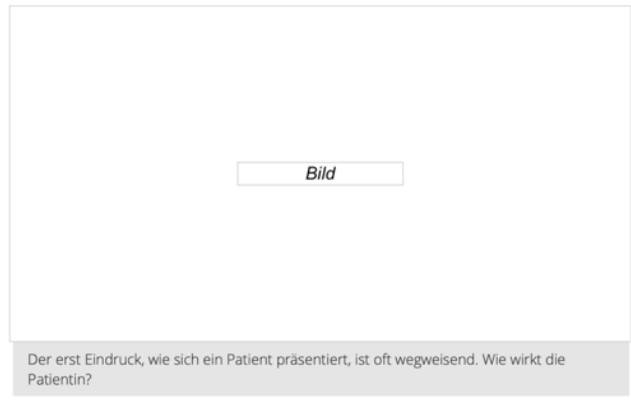

Der erst Einaru
Patientin?
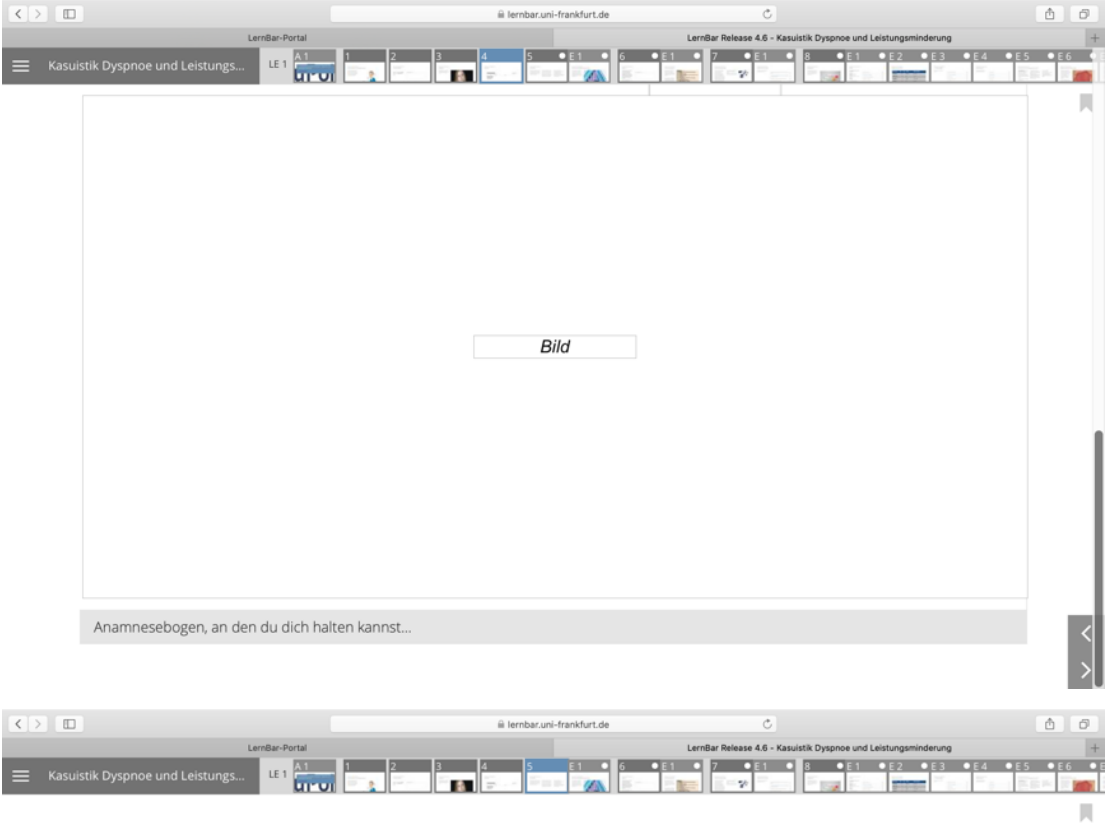

Kasuistik Dyspnoe und Leistungsminderung

Die Patientin...

Unsere Patientin heilst Maria Schmidt und

ist 27 Jahre alt. Dir allt auf, dass sie etwas

offene Frage hin was sie denn zum Arz

"Ich bekomme in letzter Zeit, wenn ich

michnict ment so fit wie sonst. Auch

eergauf fahren muss, bekomme ich sehr

schnell zu wenig Lutt... Und tagsuuber bin

zur Ärztin führe, antwortet sie:

ich etwas schlapp und oft sehr müde:

nese: Sie raucht nicht,

trinkt nur gelegentilich Alkoho.

Frau Schmidt leidet an keinen

Asthma. Außerdem nehme die Patientin

keine Medikamente ein... 


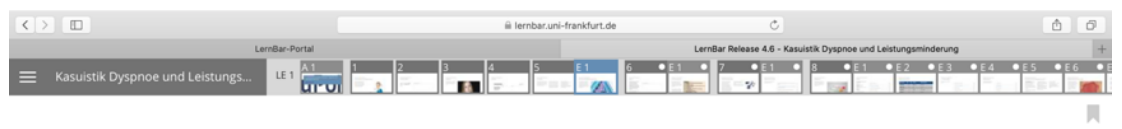

Kasuistik Dyspnoe und Leistungsminderung

Achtung bei der Medikamentenanamnese...

Bei (jungen) Frauen wird oft vergessen nach

selbsterwähnen diges nicht Trotzdem

gehören die Kontrazeptiva zur

Medikamentenanamnese. Auch deine

Patientin Maria Schmidt nimmt ein orales

Kontrazeptivum ein

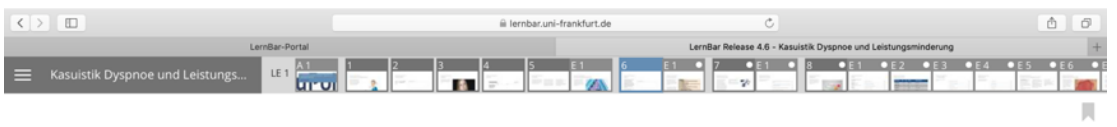

Kasuistik Dyspnoe und Leistungsminderung

Dyspnoe

Als Dyspnoe (oder auch Luft-/Atemnot)

wird das subjektive Gefuhi einer

Atmung bezeichnet. Sie kann mit

objektiven Zeichen wie z.B. dem Einsa

der Atemhilfsmuskulatur, dem Zwang zur

aufrechten Körperhaltung (Orthopnoe)

und einer erhöhten Atemfrequenz

Nenne die 4 Hauptorgansysteme, die eine Dyspnoe verursachen könnten.
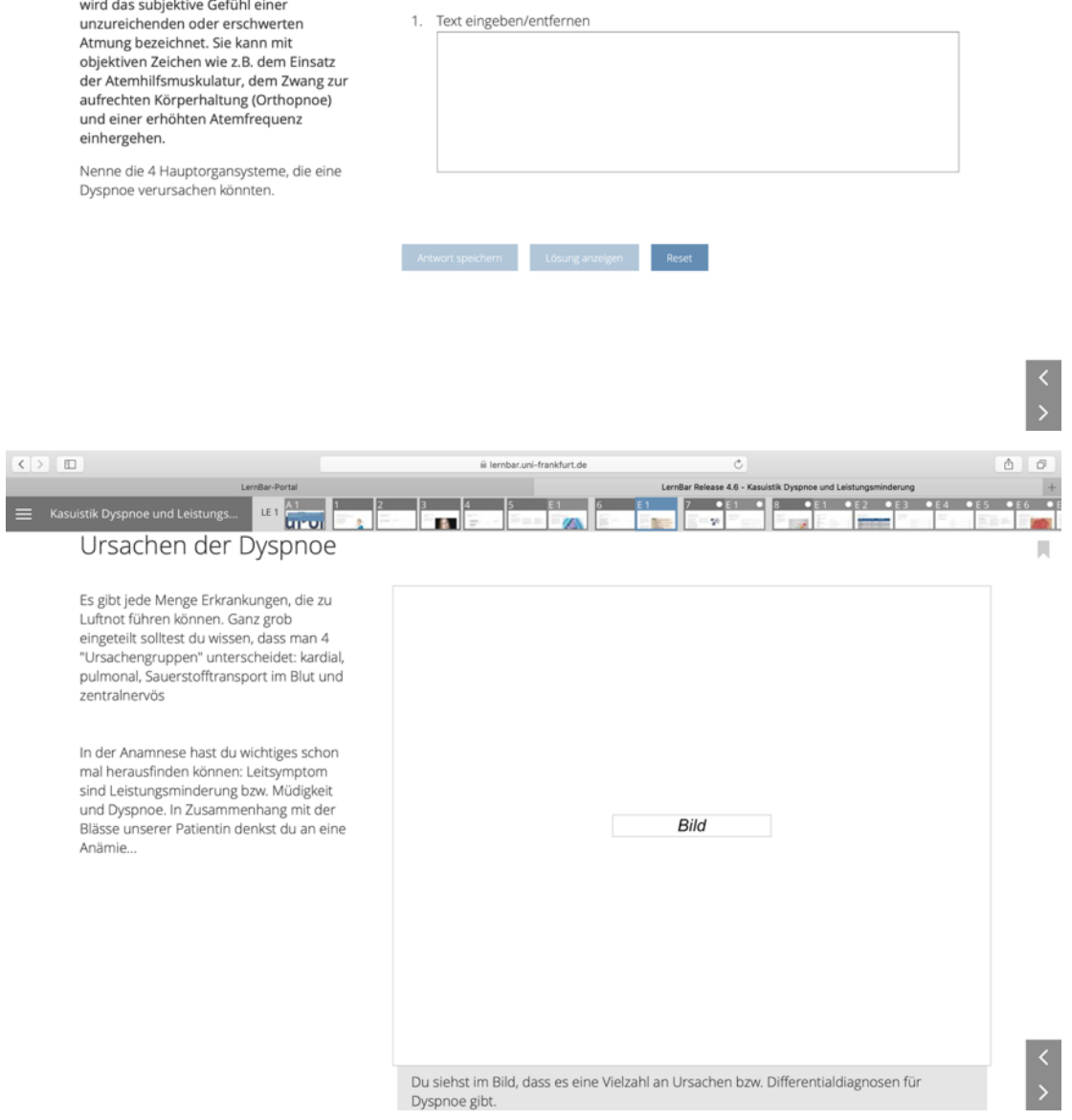


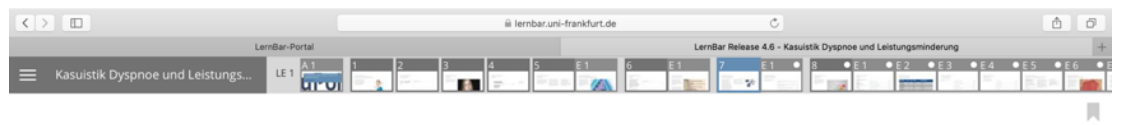

Kasuistik Dyspnoe und Leistungsminderung

Körperliche Untersuchung

Ganz nach dem klassischen Schema

kommen nach deiner Anamnese

Das Herz ist genauso wie Lunge unauffallig

reine rhythmische Herztöne ohne Vitien,

beidseitig vesikuläres Atemgeràusch ohne

Rasselgeräusche. Das Abdomen ist weich,

keine Druckdolenzen oder Resistenzen zu

tasten. Ou erkennst 3 kleine Narben

Unterbauch) die für enine A Appendeltomie

sprechen, welche die Patientin bestätigt.

Die Extremitäten sind frei beweglich die

Pulse tastbar und keine Ödeme zu

erkennen.

Puls ist rhythmisch und liegt bei $110 / \mathrm{min}$.

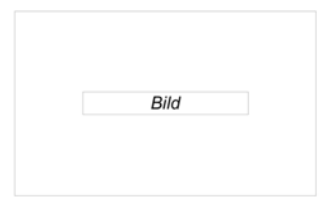




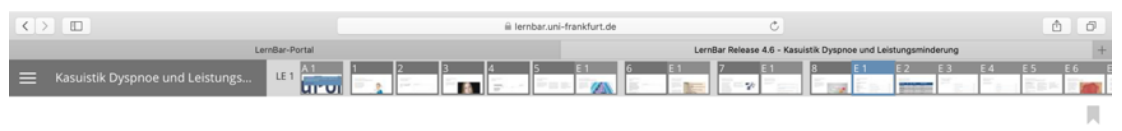

Kasuistik Dyspnoe und Leistungsminderung

Blutbild

Folgende Parameter gehören zu einem

keinen Blutbild:

\begin{tabular}{lc} 
1. Kleines Blutbild + Blutkultur & 0 \\
\hline 2. Kleines Blutbild + Differenzialblutbild & 0 \\
\hline 3. Kleines Blutbild + Gerinnungsparameter & 0 \\
\hline 4. Kleines Blutbild + Herzenzyme & 0 \\
\hline 5. Kleines Blutbild + Retikulozyten & 0
\end{tabular}

Hämatokrit

Erythrozytenindices (MCH (G), MCV (G),

$\operatorname{MCHC}(G))$

Thrombozyten

Was ist aber ein großes Blutbild?

5. Kleines Blutbild + Retikulozyten

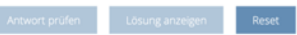

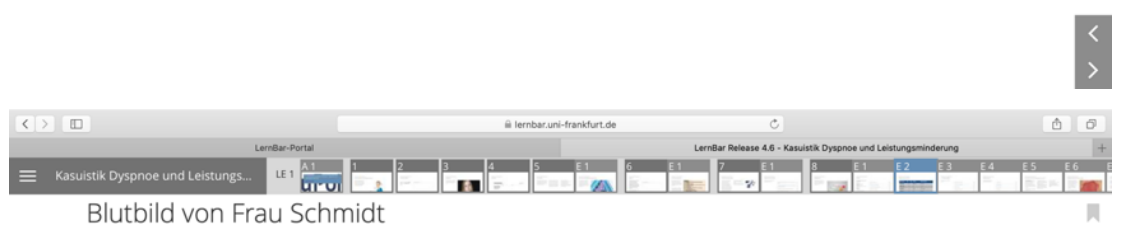

Bild
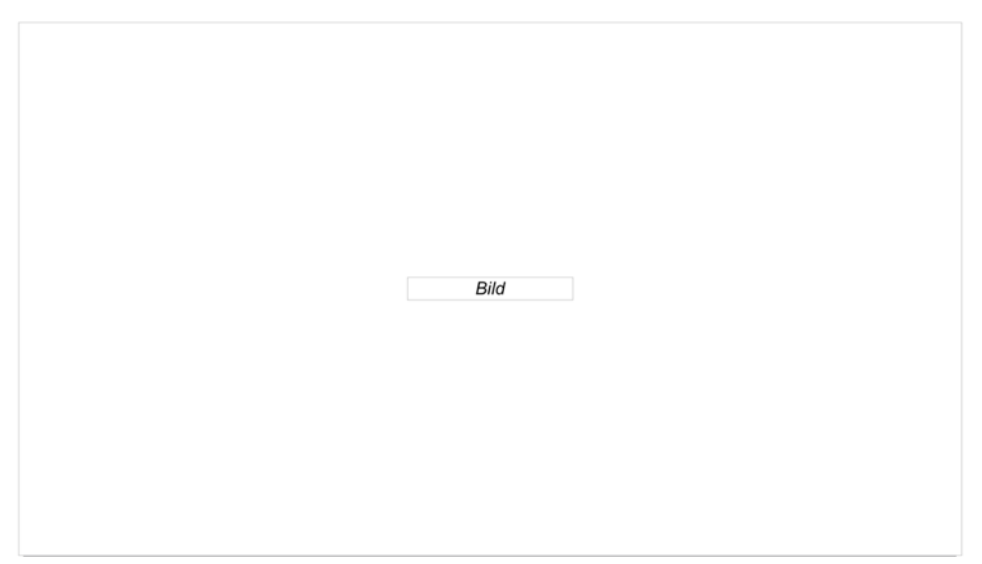

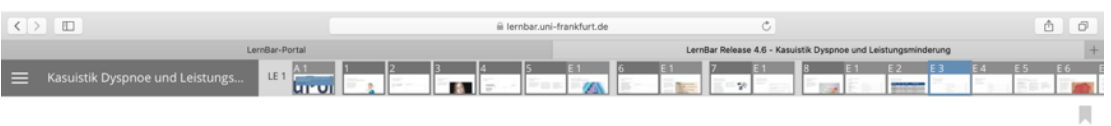

Kasuistik Dyspnoe und Leistungsminderung

... und das bedeutet?

Was diagnostizierst du aus diesem

\begin{tabular}{lc} 
1. Makrozyäre hyperchrome Anämie & 0 \\
\hline 2. Normochrome normozytäre Anämie & 0 \\
\hline 3. Hämolytische Anämie & 0 \\
\hline 4. mikrozyăre hypochrome Anämie & 0 \\
\hline 5. Renale Anämie & 0
\end{tabular}

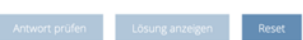




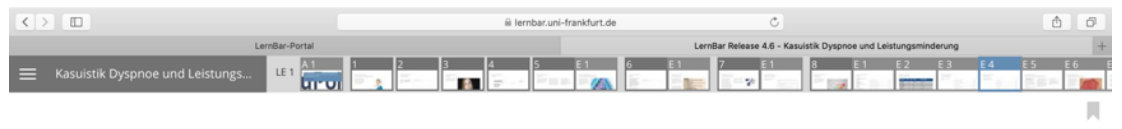

Kasuistik Dyspnoe und Leistungsminderung

häufige Ursachen

Was ist die häufigste Ursache fưr eine

mirkorä̌re hypochrome Anämie?

\begin{tabular}{lc} 
1. Folsäuremangel & 0 \\
\hline 2. Hämoglobinmangel & 0 \\
\hline 3. Vitamin B12 Mangel & 0 \\
\hline 4. Calciummangel & 0 \\
\hline 5. Eisenmangel & 0
\end{tabular}

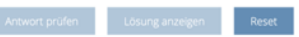

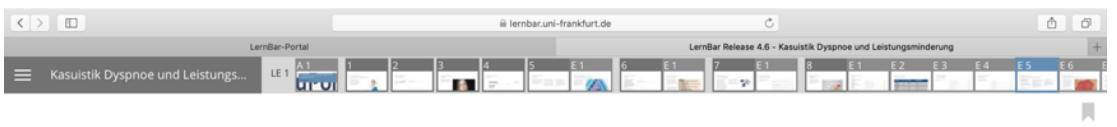

Kasuistik Dyspnoe und Leistungsminderung

Was hat es mit der Eisenmangelanämie auf sich?

Die Eisenmangelanämie ist weltweit die

häuffigste Mangelerkrankung der

Gesamtkörpereisens + eine Anämie if:

$\mathrm{Hb}<12 \mathrm{~g} / \mathrm{dl}$, 字 $\mathrm{Hb}<13 \mathrm{~g} / \mathrm{d})$. Sie ist meisten

mikrozytär und hypochrom (MCH und MCV

sind niedrig).

Es gibt zwei Hauptursachen, die zu diesem

Krankheitsbild führen
1. Mangelhatte Eisenaufnahme Mangende Zufunr, mangelnde Resorption mögliche Ursachen. In der

Schwangerschaft $7 \mathrm{~B}$ besteht ein erhöhter

Eisenbedar. Eine mangelnde Zufuhr ist

eher selten anzutreffen, könnte aber

beispielsweise bei streng vegan lebenden
2. Chronischer Blutverlust

Menstruationsblutung bei frauen denken

da dies der hāufigste Grund der

Eisenmangelanämie ist. Aber auch
gastrointestinale Blutungen darfst du nich

vergessen!

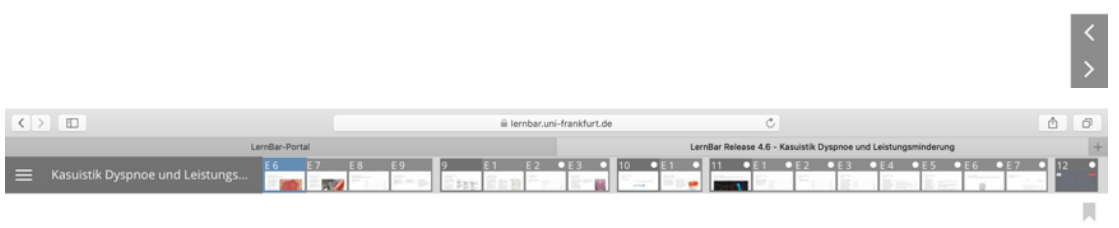

Kasuistik Dyspnoe und Leistungsminderung

Mögliche Symptome bei Eisenmangel

Die Mundschleimhaut kann bei einer

Eisennmangetanamie verändert sein, man

spricht

- Atrophie der Mundschleimhaut (au

Pharynx und Osophagus)

- brennende Zunge und Dysphagie
- auch Mundwinkelrhagaden sind

wegweisend

Bei einer Elsenmangelanämie kann es

Nagel- und Haarveränderungen geben, wie

uchigkeit und Haarausfall

möglich.

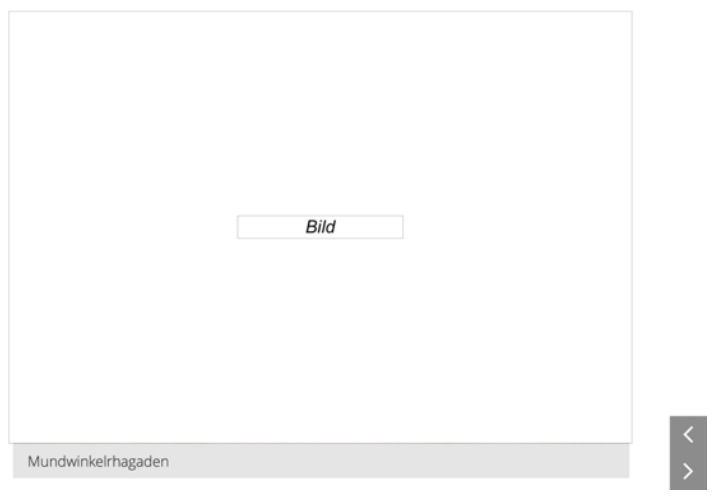




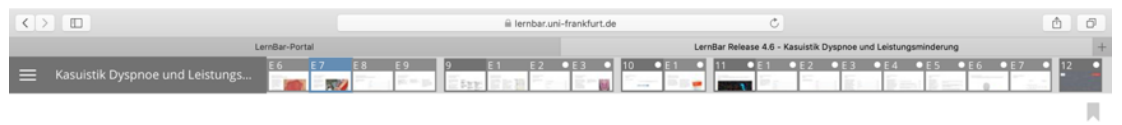

kasuistik Dyspnoe und Leistungsminderung

Zurück zur Patientin

Nun hat sich unser Verdacht nach der

körperlichen Untersuchung und mit der

bei der Patientin genauer nach.

Frau Schmidt berichtet nun auch, dass ihre

letzte Regelblutung etwas starker war als

beim Blutspenden.

Bild

Blutspenden sollte man in der Anamnese (bei Verdacht auf Anämien) abfragen.

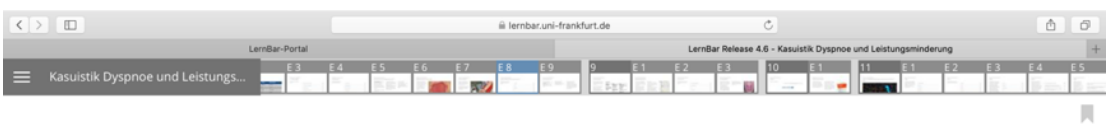

Kasuistik Dyspnoe und Leistungsminderung

Welchen Blutwert kannst du noch bestimmen?

Welchen Wert solltest du dir in der

\begin{tabular}{lc} 
1. Transferrin & 0 \\
\hline 2. Ferritin & 0 \\
\hline 3. Serumeisen & 0 \\
\hline 4. CRP & 0 \\
\hline 5. BSG (Blutsenkungsgeschwindigkeit) & 0
\end{tabular}

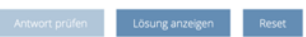

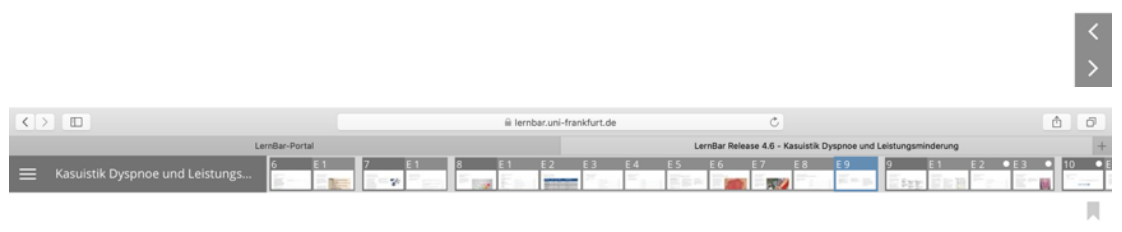

Kasuistik Dyspnoe und Leistungsminderung

Das schaust du dir an:

Super! Du schaust dir bei der

tisenmangelanamie am beste

Ferntin- Wert an, dieser ist

Parameter und sensitiver als z.B. das

Der Ferritinwert erlaubt eine

Unterscheidung zwischen einer

Eisenmangetanamie (Ferritin $\downarrow)$ und en

Mernen solmest du dir: Eine Fernitin

Enriedrigung ber erniedrigter

Eisenmagenganzentration ist fur eine

Jedoch darfst du eine Eisenmangelanămie

nicht ausschließen, wenn der Ferritin- Wert

nicht erniedrigt ist (ist also sehr sensitiv.

aber gering spezifisch. 


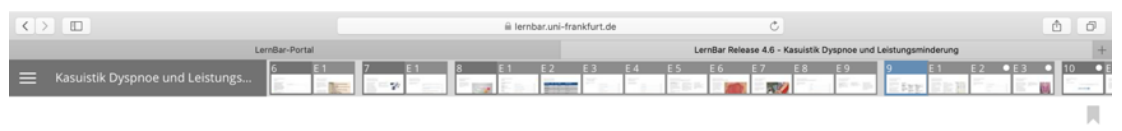

Kasuistik Dyspnoe und Leistungsminderung

Perniziöse Anämie

Nun haben wir eine Form der Anămien besprochen, es git jedoch noch andere haben solltest.

Das MCV (G) (= mittleres korpuskuläres

Volumen) und $\mathrm{MCH}(\mathrm{G})$ (= mittlerer

korpuskularer Hb Gehatt) sind sogenannte

Erythrozytenindices. Sie korreileren

Differenzierung der Anämien weiter.

\begin{tabular}{|c|c|c|}
\hline zu niedrig & normal & zu hoch \\
\hline $\begin{array}{l}\text { Ferritin niedrig: } \\
\text { Eisenmangel } \\
\text { Ferritin normal/ erhöht: } \\
\text { Anämie bei chronischer } \\
\text { Erkrankung } \\
\text { Thalassämie }\end{array}$ & $\begin{array}{l}\text { Retikulozyten niedrig: } \\
\text {-renale Anămie } \\
\text { - aplastische Anămie } \\
\text {-seltene Formen } \\
\text { Retikulozyten normal: } \\
\text { Anämie bei chronischer } \\
\text { Erkrankung } \\
\text { Retikulozyten erhöht: } \\
\text { - Hämolyse } \\
\text { - Blutung } \\
\text { - Regeneration }\end{array}$ & 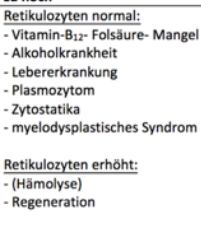 \\
\hline
\end{tabular}

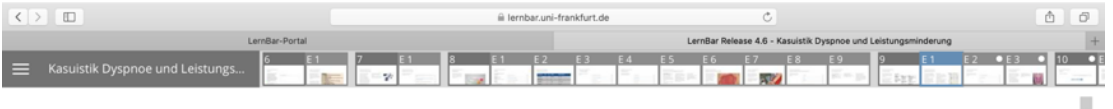

Kasuistik Dyspnoe und Leistungsminderung

Megaloblastäre Anämien

Die Ursachen für eine megaloblastăre

Anamie sind Vutamin B12-Manget oder

und hyperchrom, D $\mathrm{MO}(\mathrm{G})$ und $M \mathrm{CH}^{\circ}$ (G)

sind erhöht

Folsäure und Vitamin B12 sind für die

Zellteilung notwendig. Durch Mange

kommt es zu einer verminderten Zellteilung

(mit DNA- Synthesestorung), wovon alle

(Panzytopenie)

Folsäuremangel:

Usthen honnen eine verminderte

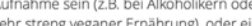

erhöhter Bedarf wie in der

Schwangerschaft

Ein weiterer wichtiger Grund (und auch vie

häufigerer) kann die Malassimilation im

Ranmen eines Mangels des Intrinsic

Resorption von Vitamin B12 im terminalten

Bild

lleum.

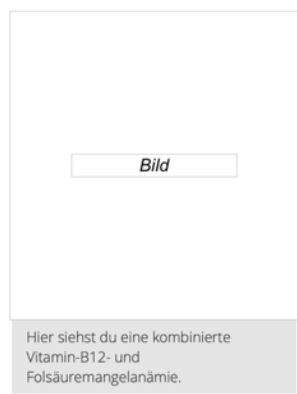

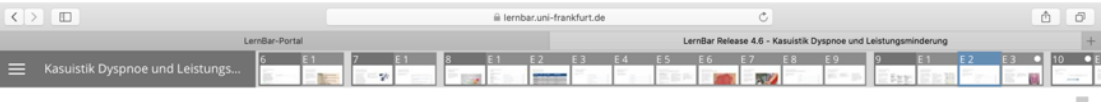

Kasuistik Dyspnoe und Leistungsminderung

Intrinsic Factor?

Womit ist die makrozytäre, hyperchrom

(G)assoziert?

\begin{tabular}{ll} 
1. Bronchial-Ca (paraneoplastisch) & O \\
\hline 2. Hämolytische Anämie & 0 \\
\hline 3. HIV & 0 \\
\hline 4. Typ-A-Gastritis & 0
\end{tabular}

4. Typ-A-Gastritits 


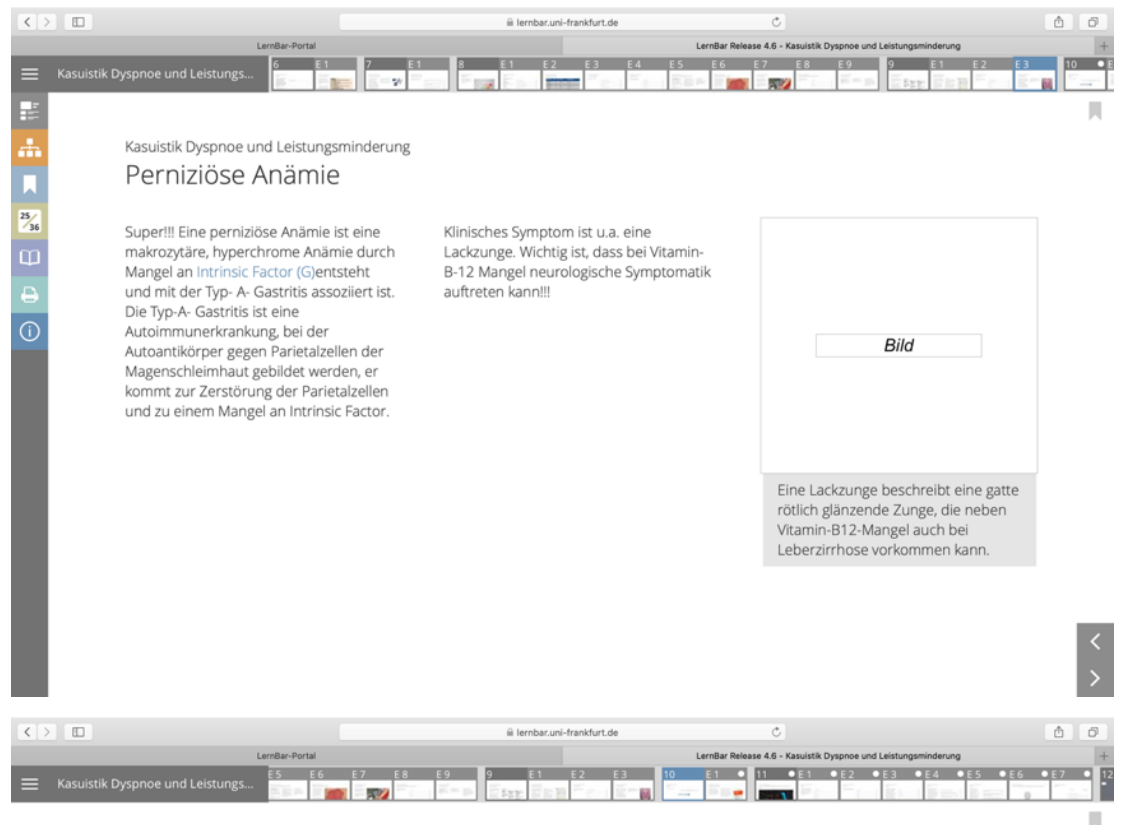

Kasuistik Dyspnoe und Leistungsminderung

Therapie

Geben Sie hier Ihren Fragetext ein.

1. Text eingeben/entfernen Text eingeben/entfernen

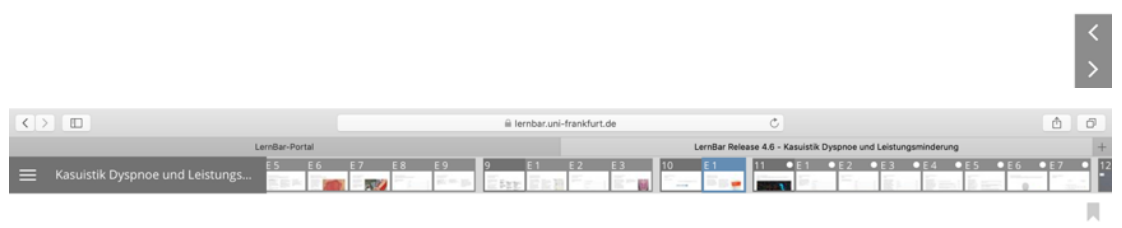

Kasuistik Dyspnoe und Leistungsminderung

Symptomatische Therapie

Ganz einfach: Es erfolgt eine orale

Eisensubstitution. Dabei ist darauf Zu

acten, ass per Ds wewertiges Eisen

Eisen kann nicht oral verabreicht werden

da es im Darm Komplexe bilden kann un

sehr viel schlechter resorbiert wird.

Um den Therapieerfolg zu beobachter

bestellst du die Patientin in ca. 2 Wochen

noch ent erneut dine Sprechstunde ein und

dann noch einmal die wichtigen Parameter

(Cave: es kann sein, dass der Hb erst ein

bisschen später ansteigt, daher ist es nich

falsch, die Patientin evil. noch ein drittes

Bild

mal einzubestellen. 


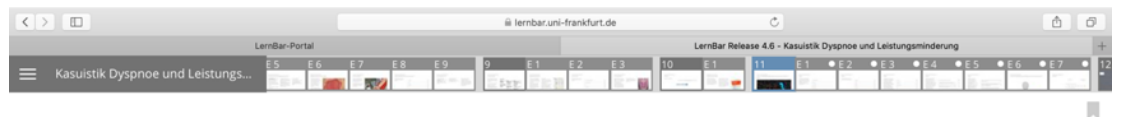

Kasuistik Dyspnoe und Leistungsminderung

Super, nun ist die Kasuistik abgeschlossen. Zum Schluss noch 5 Fragen zum üben :)

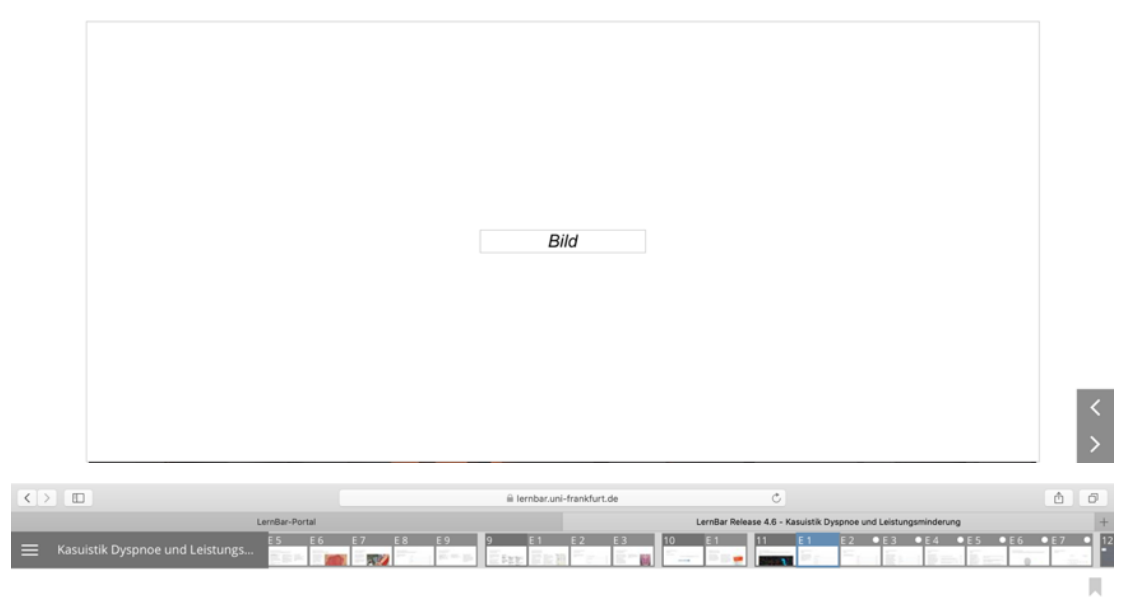

Kasuistik Dyspnoe und Leistungsminderung

Frage 1

Ein 5 Monate atter voll gestillter Säugling

taitt durch eine blasse Hautrarbe aut. Die

beträgt 91 gl Das MC betrögt 64 fl Die

Serumferritinkonzentration ist

vermindert.Was ist die wahrscheinlichste

\begin{tabular}{lc} 
1. Vitamin-B12-Mangel & 0 \\
\hline 2. Eisenmangel & 0 \\
\hline 3. Folsäuremangel & 0 \\
\hline 4. Kugelzellanămie & 0 \\
\hline 5. Thalassaemia major & 0
\end{tabular}

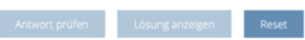

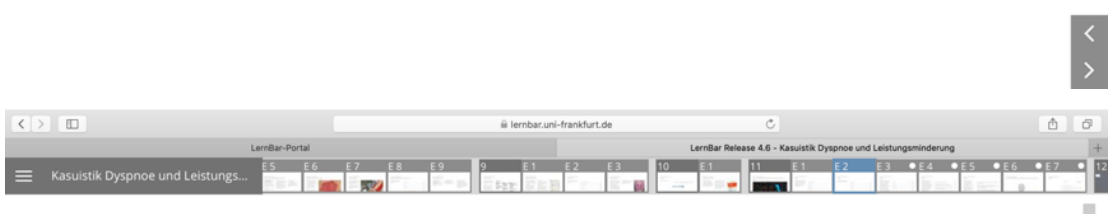

Kasuistik Dyspnoe und Leistungsminderun

Frage 2

Welches Antibiotikum gehört nicht zum

Standartherapieschema

\begin{tabular}{lc} 
1. Moxifloxacin & 0 \\
\hline 2. Isoniazid & 0 \\
\hline 3. Rifampicin & 0 \\
\hline 4. Pyrazinamid & 0 \\
\hline 5. Ethambutol & 0
\end{tabular}

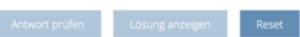




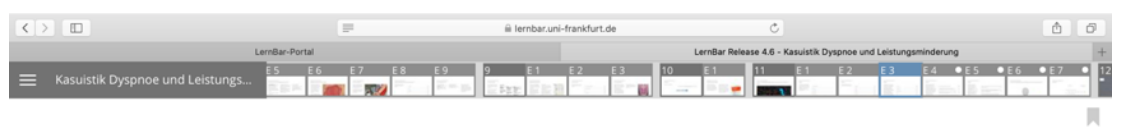

Kasuistik Dyspnoe und Leistungsminderung

Frage 3

Eine 45-järrige Patientin mit bekannter

Abklärung

einer Anämie in einer hämatologischen

Klinik vorgestellt. Sie ist seit einigen

Monaten weniger leistungsfăhig,

insbesondere bei körperlicher Belastung.
Die Polyarthritis ist derzeit mit einer relativ

niedrigen Dosis von Methotrexat eingestellt

(10 mg einmal pro Woche). Darunter

berichtet die Patientin über wechselnde

Aor der MTX-Gabe ausgepräs

seien.Klinisch zeigt sich eine blasse

Patientin mit geringen Arthritiszeichen -

Synovialitis und Überwärmung - im Bereich

der Metakarpophalangealgelenke beidseits,

sowie im inken Elenbogengelenk Welcher

Laborwert erlaubt am sichersten

Eisenmangelanämie und einer

sogenannten Anämie der chronischen

Erkrankung (Entzündungsanämie)?

\begin{tabular}{ll} 
1. Serumeisen & 0 \\
\hline 2. Haptoglobin & 0 \\
\hline 3. Retikulozytenzahl & 0 \\
\hline 4. MCV & 0 \\
\hline 5. Ferritin & 0 \\
& \\
\hline &
\end{tabular}

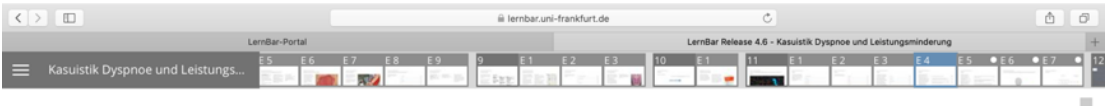

Kasuistik Dyspnoe und Leistungsminderung

Frage 4

Bei einer 44.jährigen, bisher gesunden

verkauferin wird anlasssich einer

$\mathrm{g} / \mathrm{g}$ gemessen. Die weitere klinisch.

chemische Diagnostik ergibt eine erheblich

und einge Ferritinkonzentration im Serum

Die Patientin gibt auf Nachfragen an, sich

vegetarisch zu ernähren, und berichtet, set

einiger Zeit verstärkte Monatsblutungen zu

Eisen. Der behandelinde Arzt verordnet ein

den Eisenmangel zu korrigieren. Welche der

folgenden Aussagen trifft diesbezüglich am

ehesten zu

\begin{tabular}{l} 
1. Zur oralen Therapie eignen sich Eisen-Il-Salze. \\
$\begin{array}{l}\text { 2. Bei Nüchterneinnahme des Elsenpräparates ist eine bessere } \\
\text { Magenverträglichkeit zu erwarten als bei Einnahme zu einer Mahlzeit. }\end{array}$ \\
$\begin{array}{l}\text { 3. Die Bioverfügbarkeit des Eisenpräparates lässt sich durch gleichzeitige Einnahme } \\
\text { von Aluminiumphosphat erhöhen. }\end{array}$ \\
\hline $\begin{array}{l}\text { 4. Die Bioverüugbarkeit des Eisenpräparates wird durch gleichzeitige Einnahme von } \\
\text { Ascorbinsäure (Vitamin C) erniedrigt. }\end{array}$ \\
\hline $\begin{array}{l}\text { 5. Im vorliegenden Fall wäre die Gabe von Erythropoetin vor der Eisenbehandlung } \\
\text { sinnvoll. }\end{array}$
\end{tabular}

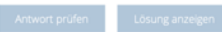

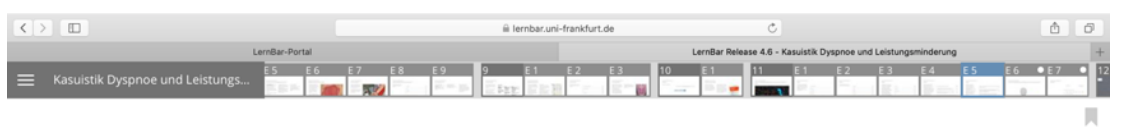

Kasuistik Dyspnoe und Leistungsminderung

Frage 5

Während eines europäischen

Notstivals ist eine pjlerin in der

Krankenhauses tätig Bei der

notfallmäßigen Aufnahmeuntersuchung

eines stark betrunken

Festivalteilnehmers aus Osteuropa hustet

dieser die PJlerin mehrmals an. Am

Folgetag entahri diese, dass be inm der

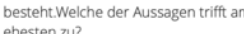

2. Die Mikroskopie von nach Ziehl- Nielsen gefarbten respiratorischen Materialien weist die höcchste diagnostische Sensitvităt von Routinemethoden zum Nachweis

3. Gemäß Infektionsschutzgesetz (IfSG) ist bereits der kinische Verdacht auf

4. Gemäß Infektionsschutzzesetz (IfSG) ist der Nachweis von Mycobacterium tuberculosis namentlich meldepflichtig (Meldepflicht des Labors).

5. Um bei der Pjlerin eine Tuberkulose auszuschließen, sind Serumentnahmen für den Antikörpernachweis direkt nach Exposition sowie drei Wochen später 


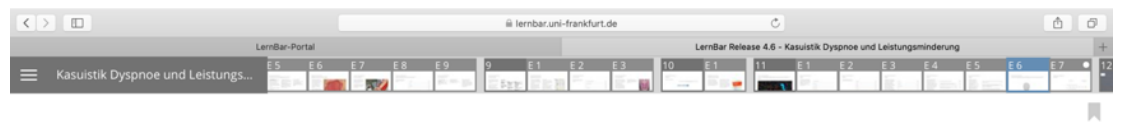

Kasuistik Dyspnoe und Leistungsminderung

Super! Du hast die Kasuistik geschafft! Bitte evaluiere noch kurz die Kasuistik!

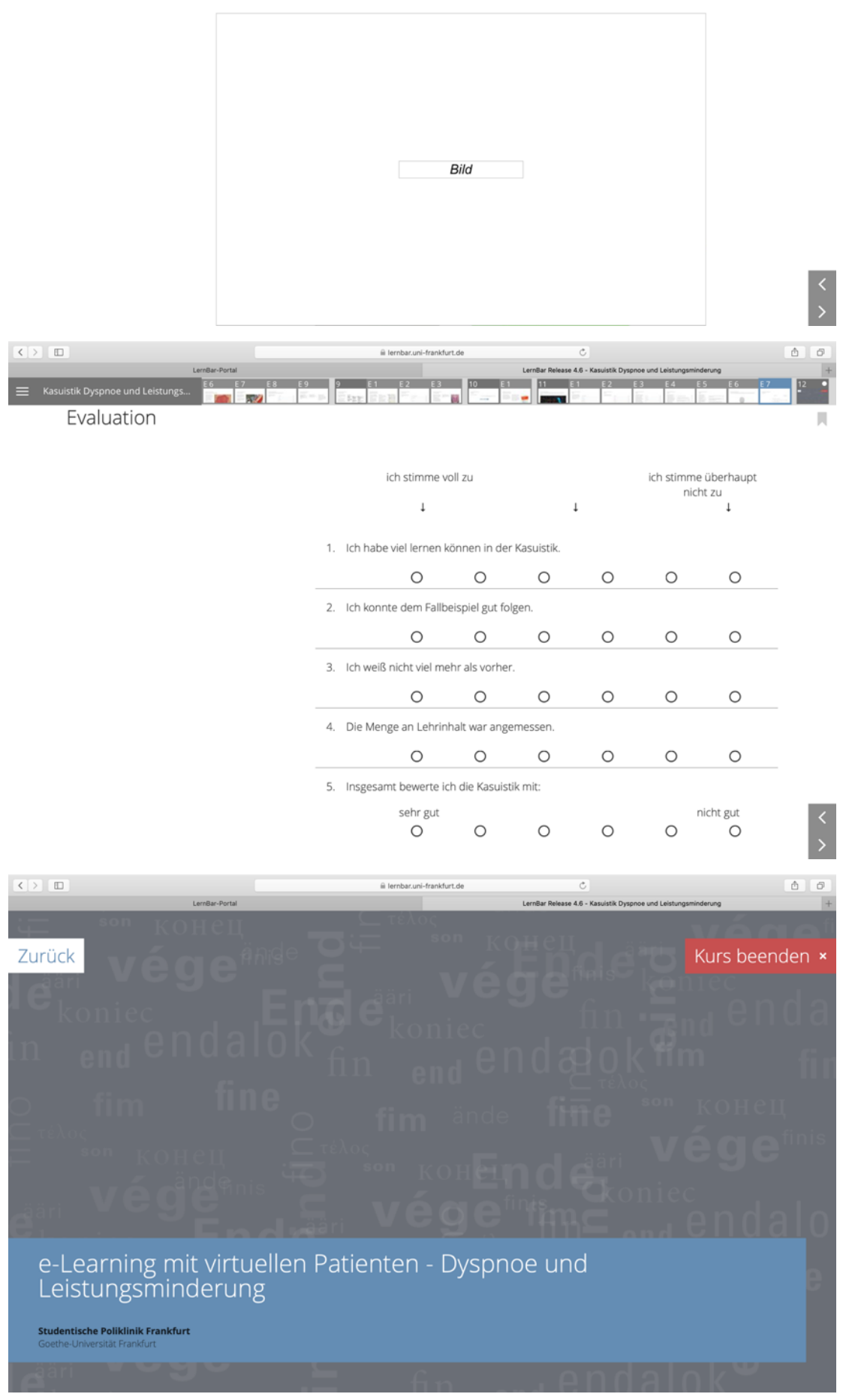


Kasuistik Kopfschmerz

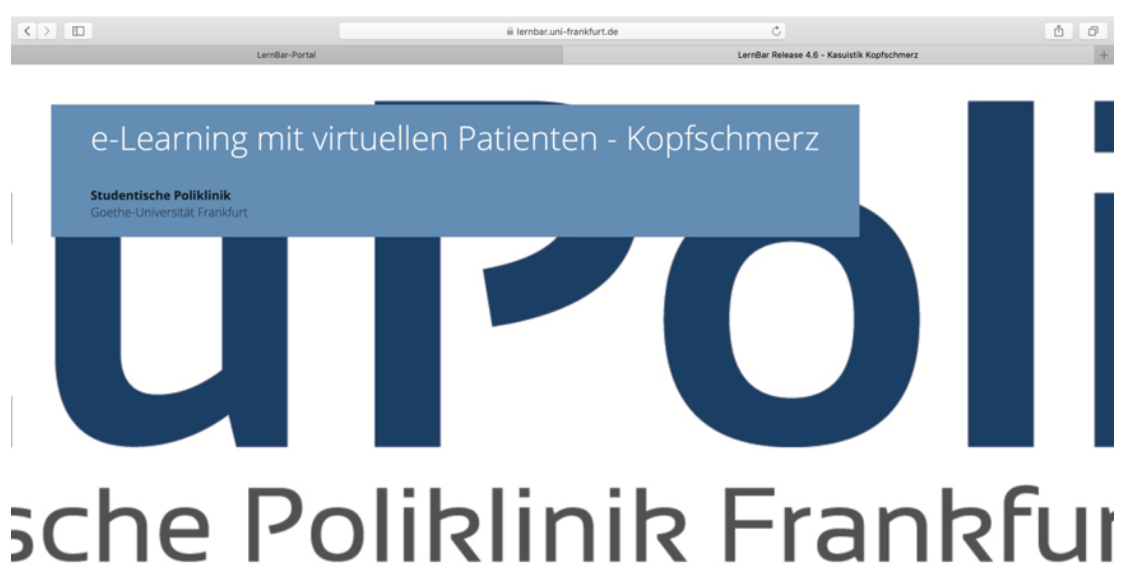

\section{Goethe Universität KURS STARTEN}

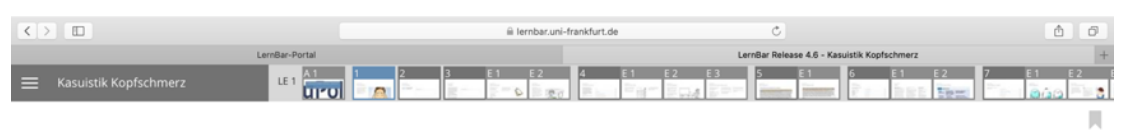

Kasuistik Kopfschmerz

Thema: Kopfschmerz

Hallo und willkommen. Heute geht es um

das Leitsymptom Kopfschmerz. In der

lernt ihr die wichtigsten

Kopterentialdiagnosen zum Primären

Therapien kennen.

Los gents mit dem Fallbeispiel!

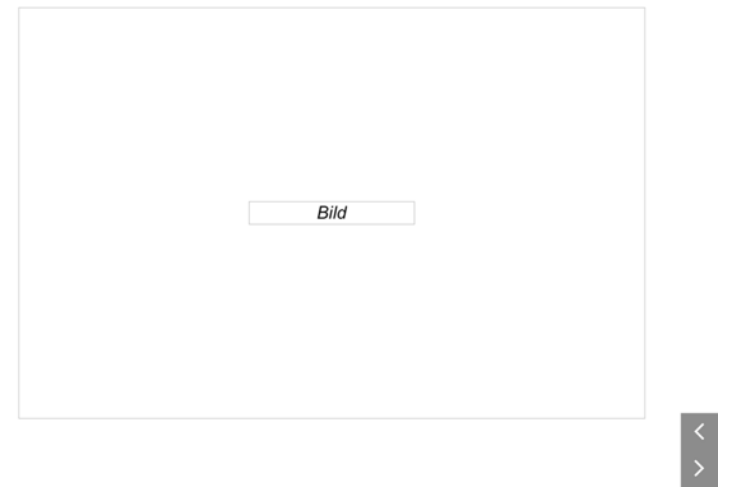




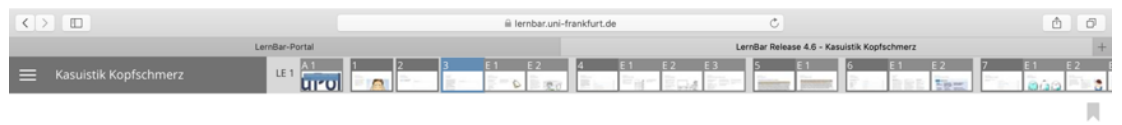

Kasuistik Kopfschmerz

Fallbeispiel

Carolin Becker (17 Jahre, w.) kommt in

deine Praxis und berichtet von einem vor

Zuerst habe ich so komisch gesehen und

danach sind mir die Wörter nicht mehr

richtig eingefallen"

Nun hat sie starke linksseitige

Kopfschmerzen und gibt an, dass si

udem berichtet sie über Übelkeit und

Das alles hat sie zum ersten mal und ist

daher sehr beunruhigt

Was ist besonders wichtig in der

Kopfschmerzanamnese?
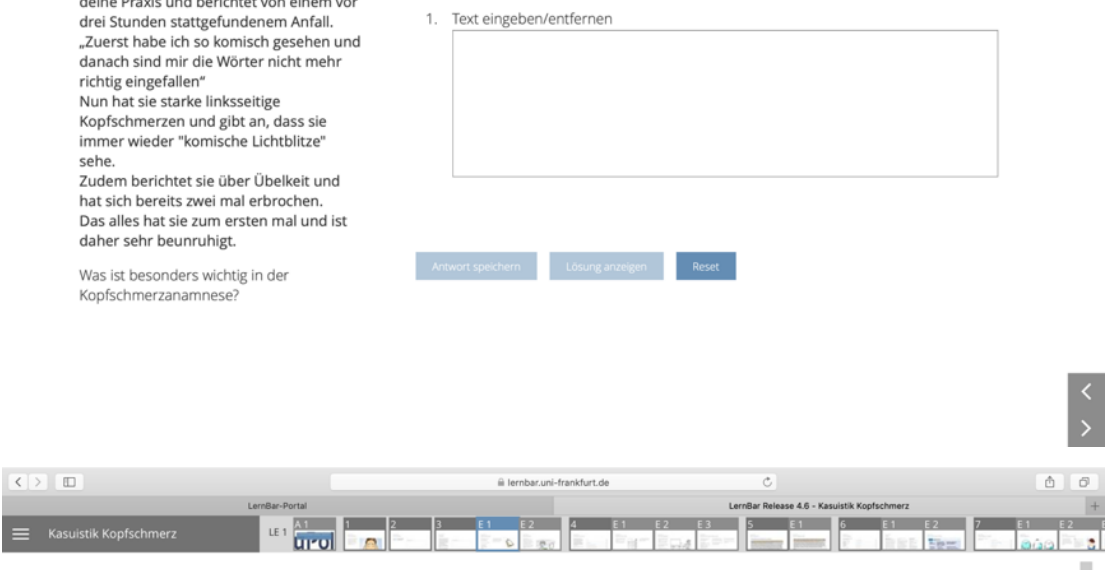

Kasuistik Kopfschmerz

Kopfschmerzanamnese

Merke dir, dass eine ausfunhrliche

Anamnese als Basis der

Zur Kopfschmerzanamnese gehören

Zeitpunkt des Auftretens

- Art des Auftretens

Dauer und Haufigkeit

- tageszetticice P

- Intensität

Charakter

Begleitsymptomatik

Verhalten wăhrend der Kopfschmerzen

Allein mit der Lokalisation oder der

Schmerzharakenstik kannst du schon später noch mal zu sprechen.
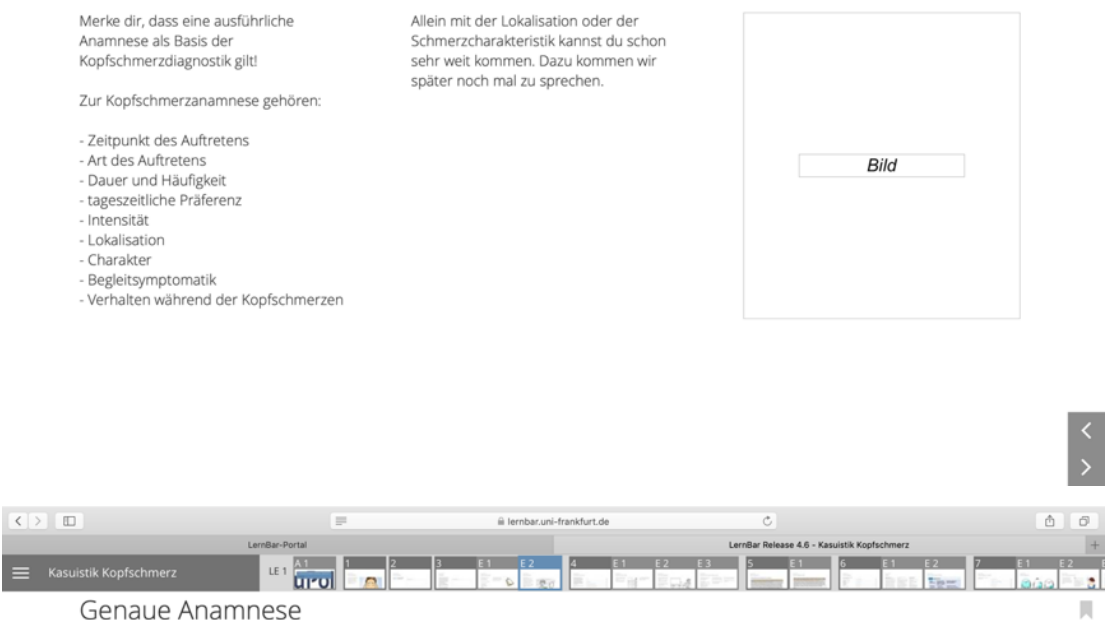

Du fragst genauer nach:

Die Schmerzen hătten vor drei Stunden

begonnen. Zuerst hatte die Pat. nur

wärent genoptsc ca ene stunde spater

seien linkssitig im Stirn und

Schläfenbereich und eine $6 / 10$ auf der VAS.

Der Schmerz sei pulsierend, bohrend und

quälend und werde durch körperliche

Anstrengung, sowie helles Licht

Bild

In der weiteren Anamnese fallt eine

Visusminderung und eine leicht

verwaschene Aussprache auf. Di

Vorerkrankun rese ist negatt.

Medikamente würden keine regelmäßri

eingenommen. Die restliche Anamnese

ergibt keine ziefführenden Ergebnisse.

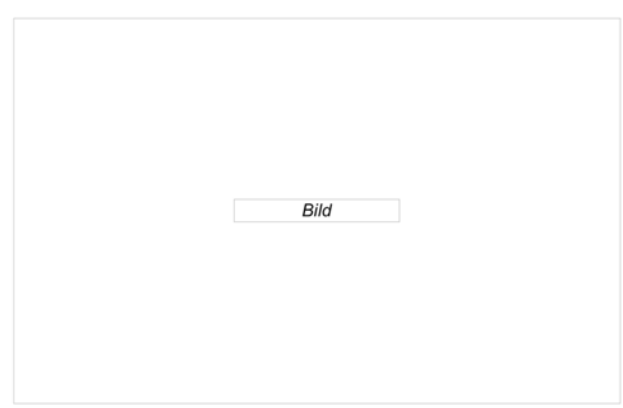

Caroline Becker berichtet auch, dass sie die Woche über Konzentrationsschwierigkeiten in der Schule hatte und ihre Hausaufgaben nicht machen konnte. Das sei ihr in der Art noch nie passiert. 


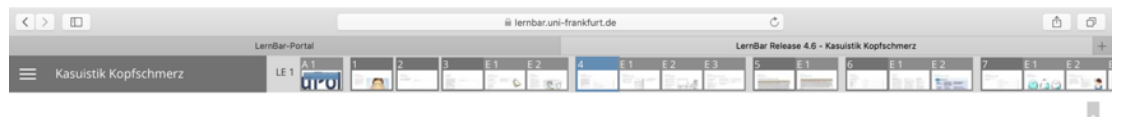

Kasuistik Kopischmerz

Körperliche Untersuchung

Du hast nun schon einen ersten Eindruck

die Anamnese hat dir schon mal sehr

weitergeholfen, du möchtest nun erst

Verdachtsdiagnose zu bestätigen und

eventuelle gravierende Erkrankungen

auszuschießen.

Welche Untersuchungen erachtest du als
sinnvollt?

\begin{tabular}{lc} 
1. EKG & $\square$ \\
\hline 2. Vitalparameter & $\square$ \\
\hline 3. grobe Neurologische Untersuchung & $\square$ \\
\hline 4. Dopplersonographie der Schläfenarterien & $\square$ \\
\hline 5. CT des Schädels & $\square$
\end{tabular}

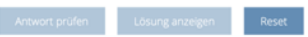

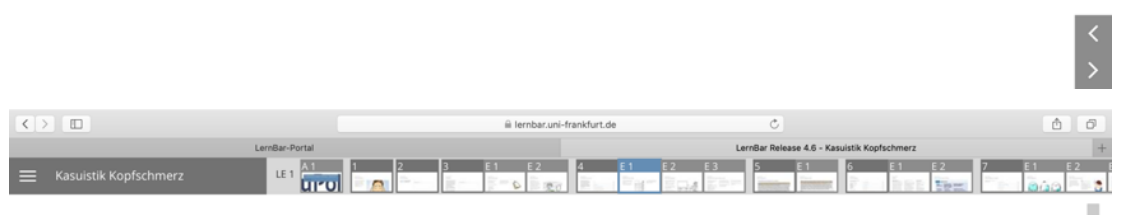

Kasuistik Kopfschmerz

Körperliche Untersuchung

Richtig sind: Vitalparameter erheben und

eine grobe neurologische $U$

Für Schädel- $C$, Doppler- Sonographie und

ein EKG gibt es keine Indikation.

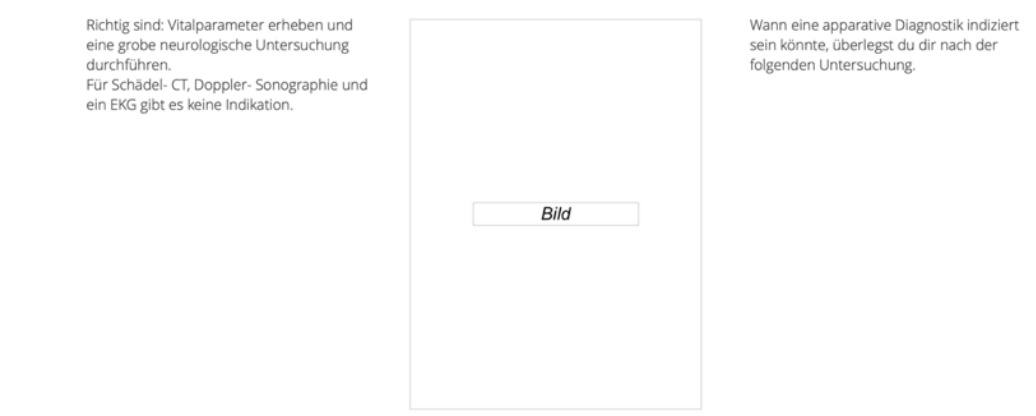

Wann eine apparative Diagnostik indizief

folgenden Untersuchung.

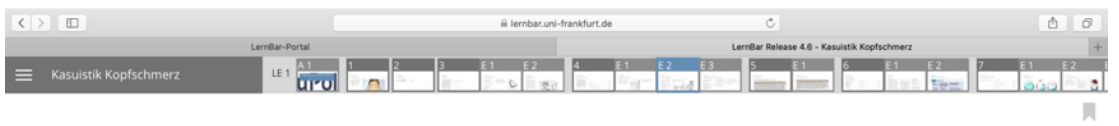

Richtig sind: Vitalparameter erheben und eine grobe neurologische Untersuchung

Für Schädel- Cr, Doppler- Sonographie und ein EKG gibt es keine Indikation.

Wann eine apparative Diagnostikindiziert sein konnte, uberlegst du dir

Hier findest du ein Video ( $(m w)$ z zur kompletten neurologischen Untersuchung

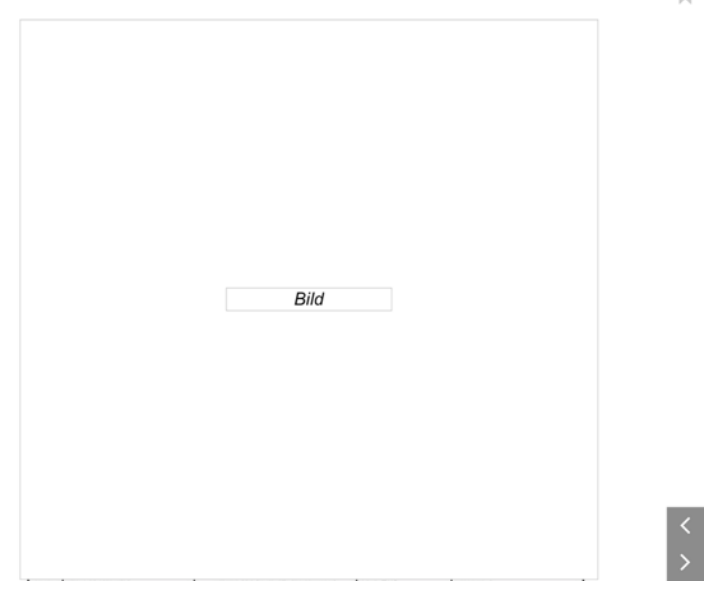




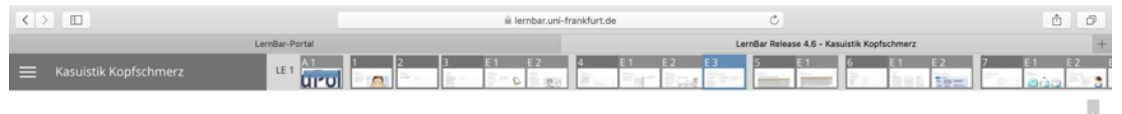

Kasuistik Kopfschmerz

Ergebnisse der körperlichen Untersuchung

Die neurologische Untersuchung und der Hirnnervenstatus ergeben keine

gischen Befund

Auch die trigeminalen

Nervenaustrittspunkte sind unauffallig. Es

ist kein Bulbusdruck- und

Bewegungsschmerz vorhanden, die HWS

ist bewegilch und auch ist kein
Weiterhin ist die Kalotte nicht klopf- und bei der Kieferöffnung hat Caroline Becker.

Ein Blick in den Mundraum zeigt dir rosige Schleimhäute und einen nicht. pathologischen Zahnstatus.
Die A temporalis lässt sich beidseitig gut tasten, es ist keine Verhărtung vorhanden. Der Blutdruck liegt bei $115 / 70 \mathrm{mmHg}$ und
der Puls bei 84 .

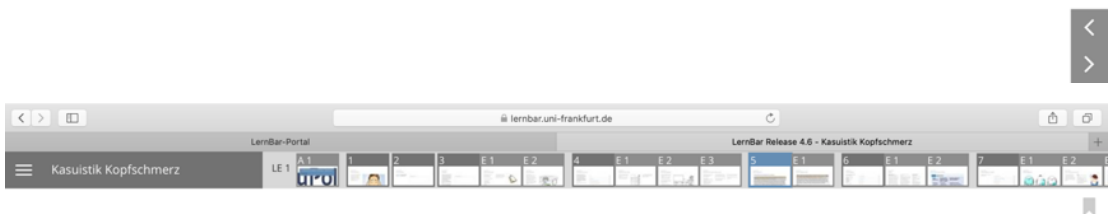

Kasuistik Kopfschmerz

Weitere Diagnostik?.

Zusammenfassung der Empfehlungen zur Diagnostik

Bild

Du hast mit der Anamnese und der körperlichen Untersuchung schon eine Menge herausgefunden.

Du hast mit der Anamnese und der körperlichen Untersuchung schon eine
Auch den Leitininien zufolge beruht die Diagnostik auf Anamnese und Klinik

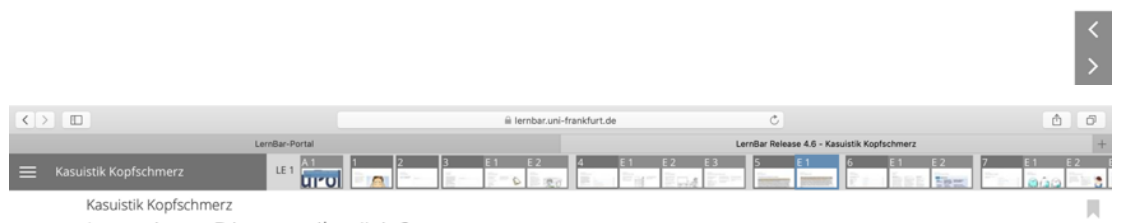

Ist weitere Diagnostik nötig? ...

Das heißt .

Zusattzliche apparative Diagnostik wird dann durchgeführt, wenn

in der Akutphase zusätzlich zum Kopfschmerz neurologische Symptomatik besteht

Red Flags ('Warnzeichen") sind:

Erstes Kopfschmerzereignis (habe eigentlich nie KS

-Schwere Kopischmerzen

U. Übelkeit und Erbrechen

Bewusstseinstörung, Fieber

Deine Patientin Caroline Becker braucht somit keine weitere Diagnostik, deine Diagnose steht fest ... 


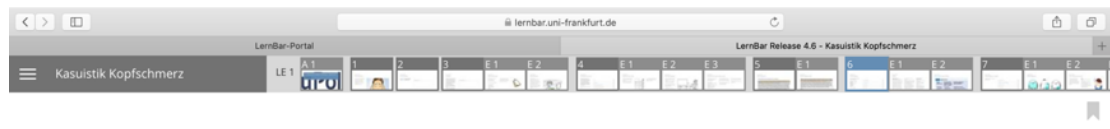

Kasuistik Kopfschmerz

Verdachtsdiagnose

Fassen wir die wesentlichen Befunde noch ein mal zusammen:

Anfallsartiger hemikranieller

Kopfschmerz

Öbelkeit und Erbrechen

Photophobie

Dysphasie

Was ist deine Diagnose?

\begin{tabular}{lc} 
1. Trigeminusneuralgie & 0 \\
\hline 2. Clusterkopfschmerz & 0 \\
\hline 3. Migräne ohne Aura & 0 \\
\hline 4. Migräne mit Aura & 0 \\
\hline 5. Seltene hereditäre, hemiplegische Migrăneform & 0
\end{tabular}

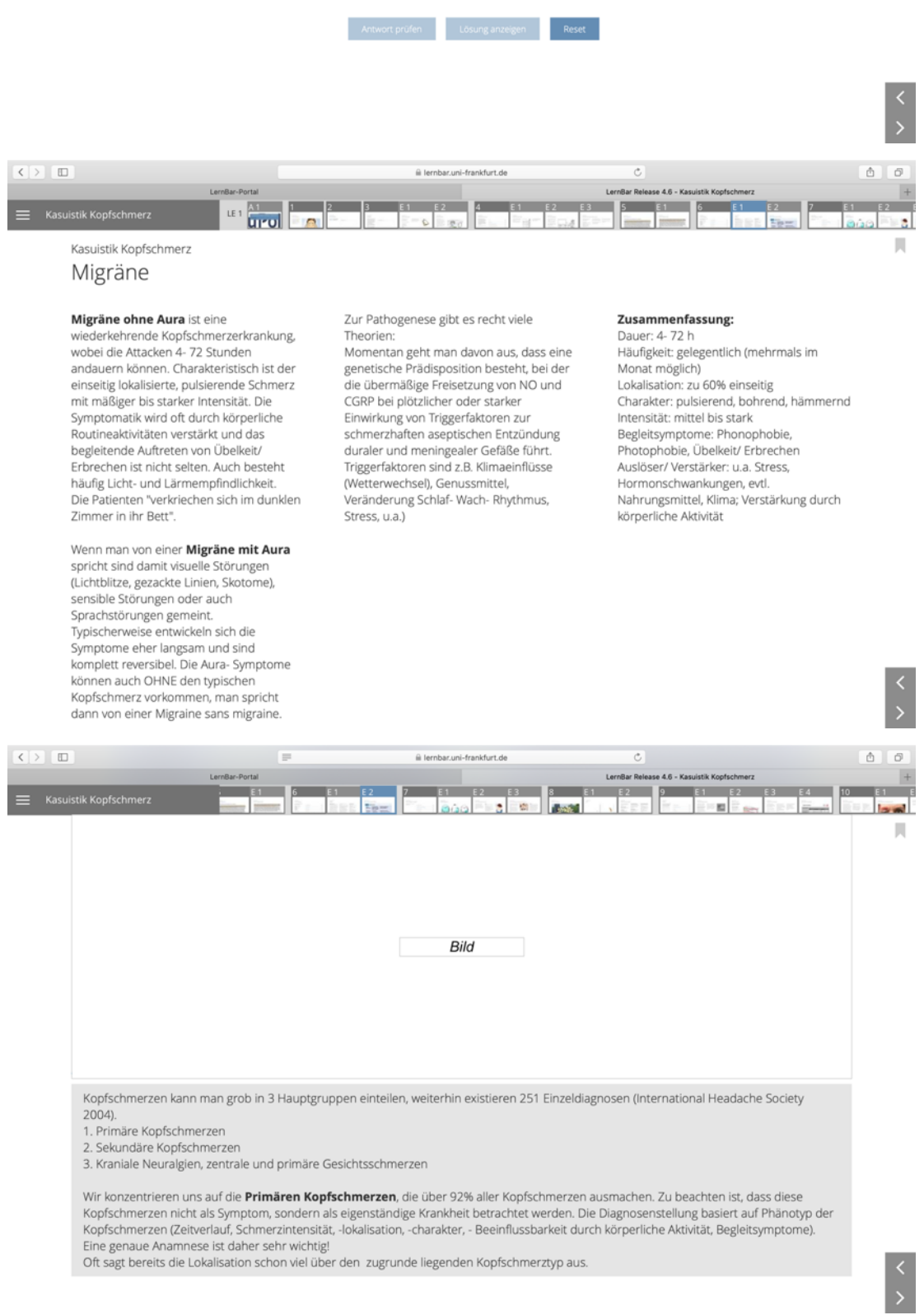




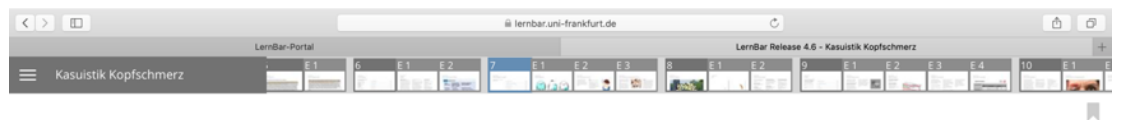

Kasuistik Kopfschmerz

Differentialdiagnosen - Migräne

Was gehört eher nicht zu einer möglichen

Differentialdiagnose?

\begin{tabular}{lc} 
1. Spannungskopfschmerz & $\square$ \\
\hline 2. Medikamenten- induzierter Kopfschmerz & $\square$ \\
\hline 3. Subarachnoidalblutung & $\square$ \\
\hline 4. Cluster- Kopfschmerz & $\square$ \\
\hline 5. Paroxysmale Hemikranie & $\square$
\end{tabular}

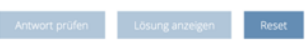
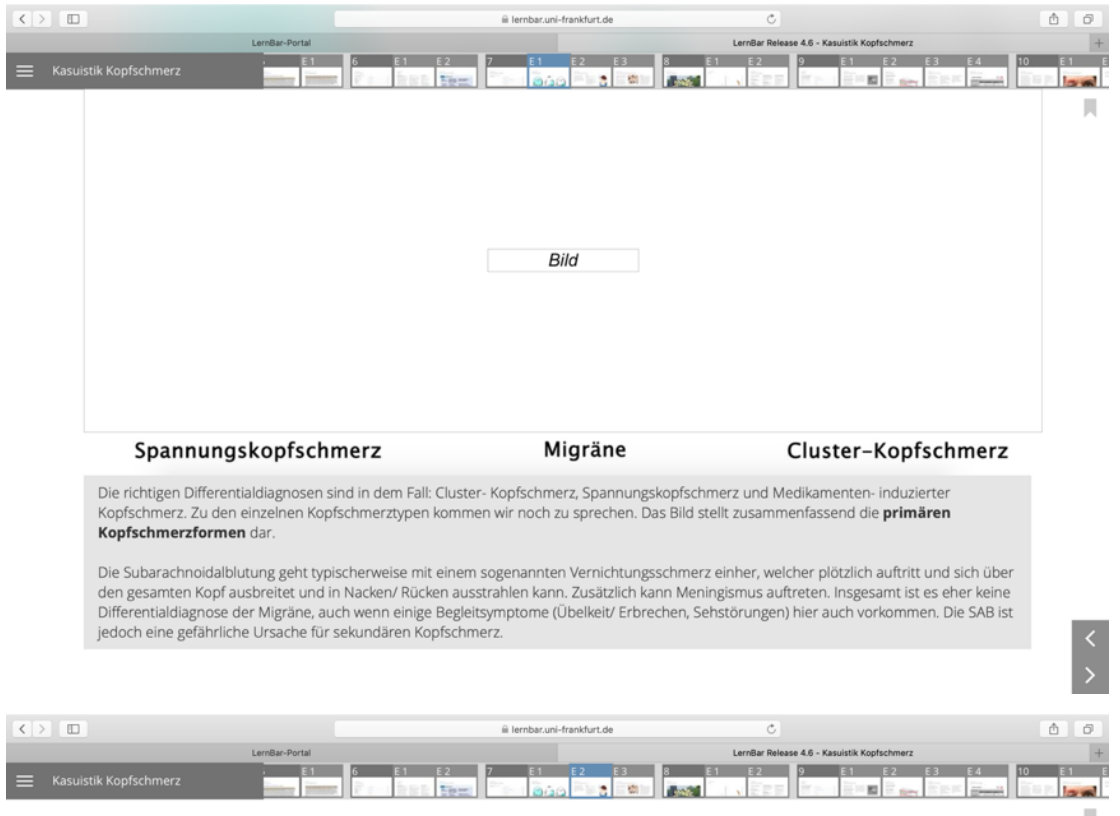

Kasuistik Kopfschmerz

Exkurs: Paroxysmale Hemikranie

Die paroxysmale Hemikranie ist ei

erz, der durch streng

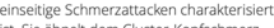

die Attacken sind jedoch in der Regel

Periorbitale bohrende Schmerzen hoher hóhere Attackenfrequenz (bis zu 30/Tag) als

beim Clusterkopischmerz

Ähnliche vegetative Begleitsymptomatik

herapie: Aufgrund der Küze der Attacke

nur prophylaktische Therape

Bild

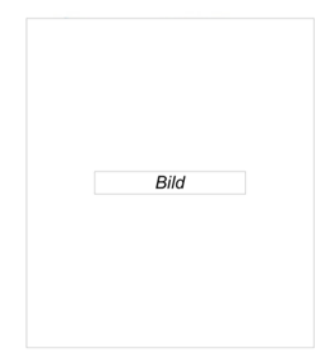




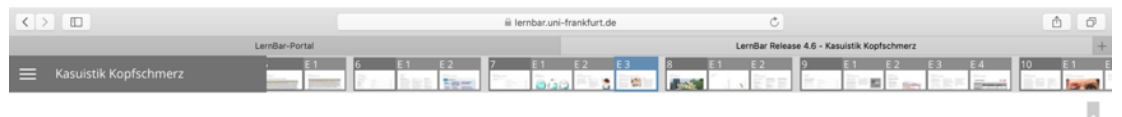

Exkurs: Medikamenten-induzierter Kopfschmerz

Die häufige Einnahme von Analgetika über mindestens 3 Monate kann zu einem

medikamenten-induzierten Kopfschmer

Medikamente können sein: Triptane

Ergotamin-Derivate, Opioide: $>10$

Tage/Monat oder: NSAR, Paracetamol und

andere Analgetika: $>15$ Tage/Monat.

Hauffy sind Patrienten betroffen, die an

der Spannungskopfschmerzen

Klinik:

- meist dumpfe 15 Tagen/Monat

.

-Müdigkeit, Ubelkeit, Lärm- und
Therapie:

Komplettes Absetzen aller Analgetika, $g$

unter stationären Bedingungen stellt die

mit Metoclopramid behandelt werden.

Auftretende Kopfschmerzen können ggt.

durch Glukokortikoide oder Neuroleptika

geindert werden. Llegt eine Migrane

Gabe von Beta-Blockern erfolgen.
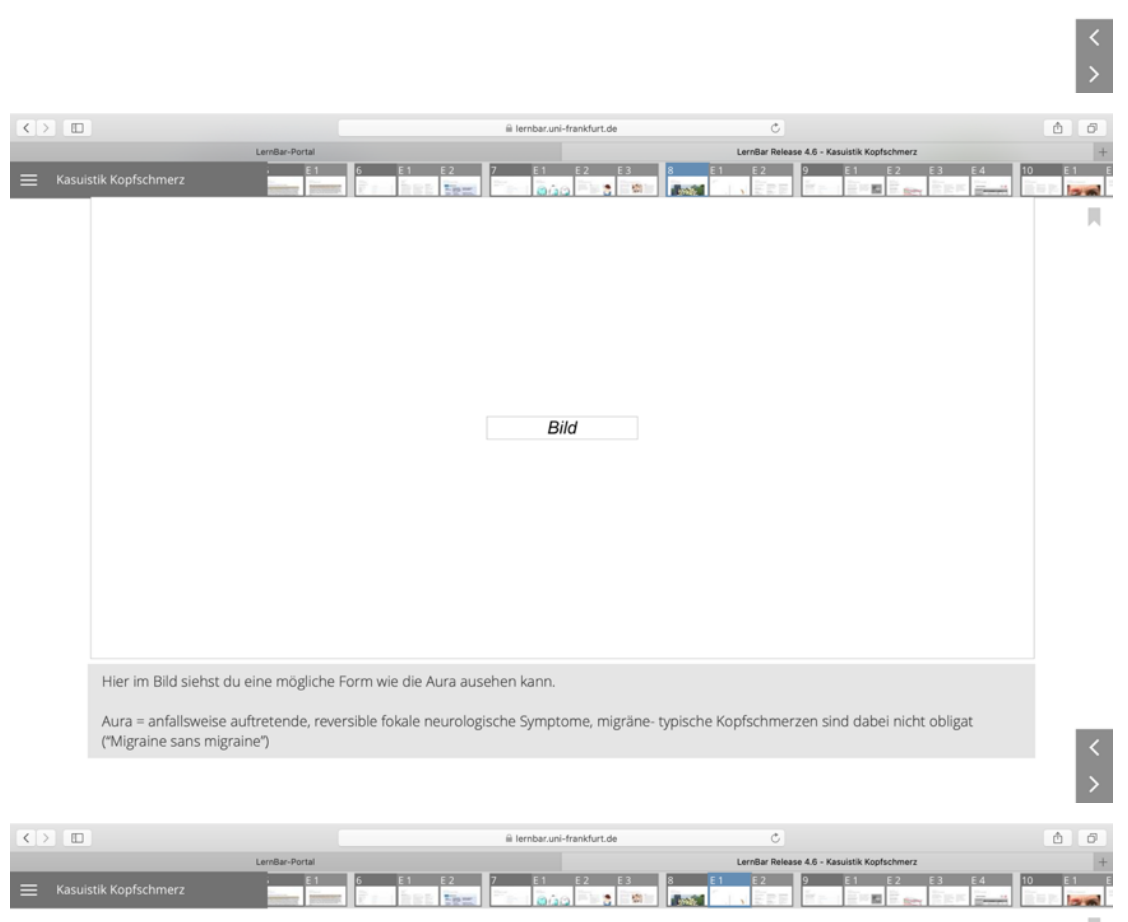

Kasuistik Kopfschmerz

Aura 


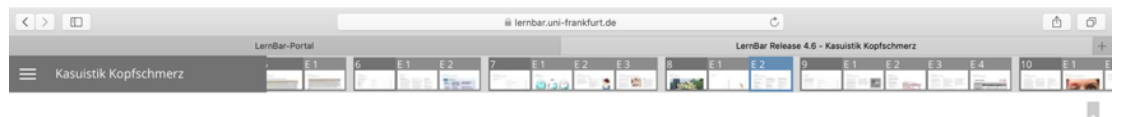

Kasuistik Kopfschmerz

Sonderformen den Migräne

Es gibt noch andere Migräneformen, die zwar eher selten sind, aber dennoch

nteressant:

- Epidemiologie: Meist Kinder betroffen ener oder mehreper Nenmerzen, Paresen

Migraine sans migraine Symptomatik vorhanden

\section{Basilarmigräne:}

Doppelbilder

Vestibuläre Migräne

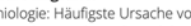

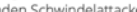

Kopfschmer

Dysarthrie, Ataxie, Hörminderung Tinnitus

Schwindel, Beidseitige Paresen oder

beidseitige Par- bzw. Hypästhesien.

mittleren Lebensalter

schwankschwindel mit Zeich-end

peripher- oder zentral-vestibulären Störun

Therapie: Zusătzl. zur Migrăne-Therapie

Dimentudvertiginosa (we bspw.

Familläre hemiplegische Migräne

Aura beinhaltet variabel ausgeprägre

mer positive Familienanamnese

Anfallsartige Dysfunktion von Cav2.1 (P/Q

type Spannungsabhangiger Calcium-Kanal,

ACMA (.2.Untereinhelt der Na/K-Pumpe),

- es handelt sich also um eine

kanalerkrankung

Relevant für uns sind jedoch die

Migrăne mit/ ohne Aura

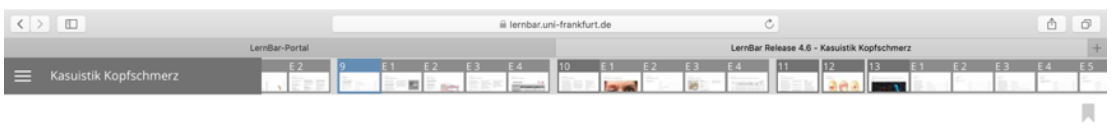

Kasuistik Kopfschmerz

Therapie

Du bist dir nun nach deiner kôrperlichen

Untersuchung und vorangegangen

hat einen akuten Migräneanfall.

Die Therapie ist somit klar. Was steht an?

Was ist bei einem akuten Migräneanfall

indiziert?

\begin{tabular}{lc} 
1. Pfefferminzöl auf die Stirn & O \\
\hline 2. Sumaptriptan und Metoclopramid & 0 \\
\hline 3. Fentanyl i.v. & 0 \\
\hline 4. $100 \%$ O2- Gabe & 0 \\
\hline 5. Metoprolol p.o. & 0
\end{tabular}

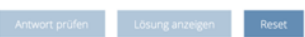

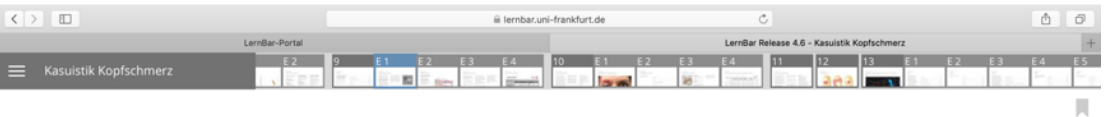

Kasuistik Kopfschmerz

Therapie der Migräne-Attacke

Eine möglichst frühe und hochdosierte Medikamenteneinnahme ist haúfig

leichte/ mittelstarke Migräneattacke

NSAR Mittel der 1 . Wahl $\rightarrow$ ASS oder

Ibuprofen (cave: Bei Kindern unter 14

Jahren ist ASS kontraindiziert, da es in

vereinzelten Fallen zum Reye-syndrom,

ener schweren Nebenwirkung, Kommen

Paracetamol)

Triptane sind 5 HT1- Rezeptor-Agonister

und fanren durch Rezeptorbindung zu

Bher vasokonshiction zerebrater

von Entzünndungsmediatoren (Substanz P.

(GRP) im Bereich der Durarterten so

kann der Schmerz im Akutfall gelindert

Bild

mittelschwere/ schwer

Migräneattacke (bei Erwachsener :

Triptane, z.B. Sumatriptan; zusătzlich immer

ein Antiemetikum. z.B. Metoclopramid 20

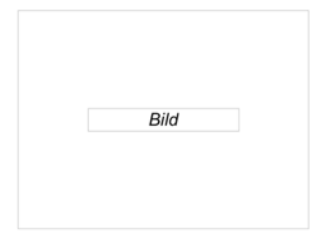




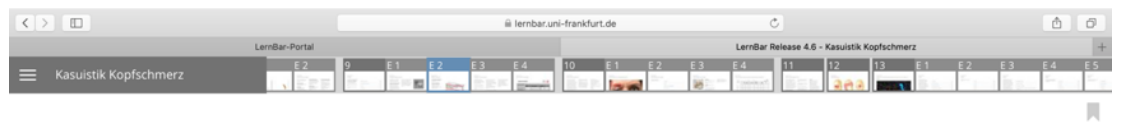

Kasuistik Kopischmerz

Migräne-Prophylaxe

nicht-medikamentöse Maßnahmen:

Lebensstiländerung, Ausdauersport

Akupunktur, u.a.

medikamentöse Maßnahmen

$\rightarrow$ Indikation: >3 Attacken/ Monat, Migräne-

Attacke $>72 \mathrm{~h}$ oder lang anhaltende

Auraphanomene

el der 1 , wahl $12 B$

Metoprolol, Propanolol)

Bild

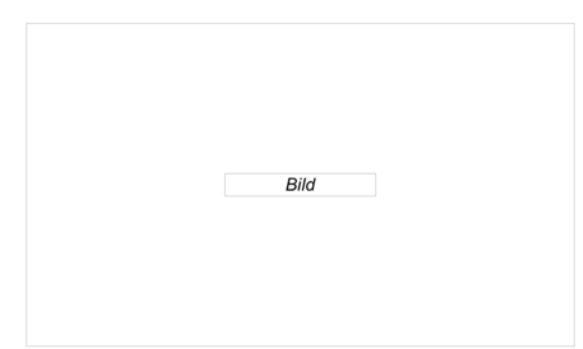

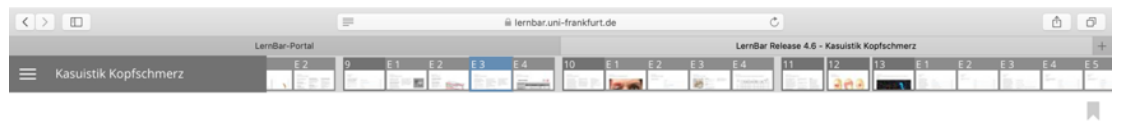

Kasuistik Kopfschmerz

Therapie von Caroline Becker...

Du legst Caroline Becker einer

intravenösen Zugang und behandelst ihren

akuten Migräneanfall mit 1000 mg ASS mg

sowie Sumatriptan i.n.

Du empfiehlst ihr zusătzlich Bettruhe un

das Vermeiden von äußeren Reizen.

Außerdem klärst du Nebenwirkungen der Triptane auf.
Innerhalb von $24 \mathrm{~h}$ nach Triptangabe kommt es in ca. 30\% d.F. zu Kopfschmerz). War die erste Triptangnicht wirksam, hilft meist eine zweite Gabe Wegen der Gefahr eines

medikamenteninduierte

Dauerkopfschmerzes sollte der Patient max. an 10 Tagen/Monat ein Triptan hehmen.

NW der Triptane sind. Flush, pektanginöse Beschwerden, Parästhesien der
Extremităten und Kätegefúhl

Kontraindikationen sind wegen Schokonstriktiver Wirkung. KHK, TIA

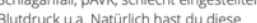
gewissenhaft schon ausgeschlossen.
Komplikationen der Migräne können sein - Status migraenosus. Ianger als 3

- Migränöser Infarkt: persistiterent

Migräne mit Aurasymptomen in

Kombination mit einem Infarktgeschehe Daher sollte man eine Migräne immer behandeln!

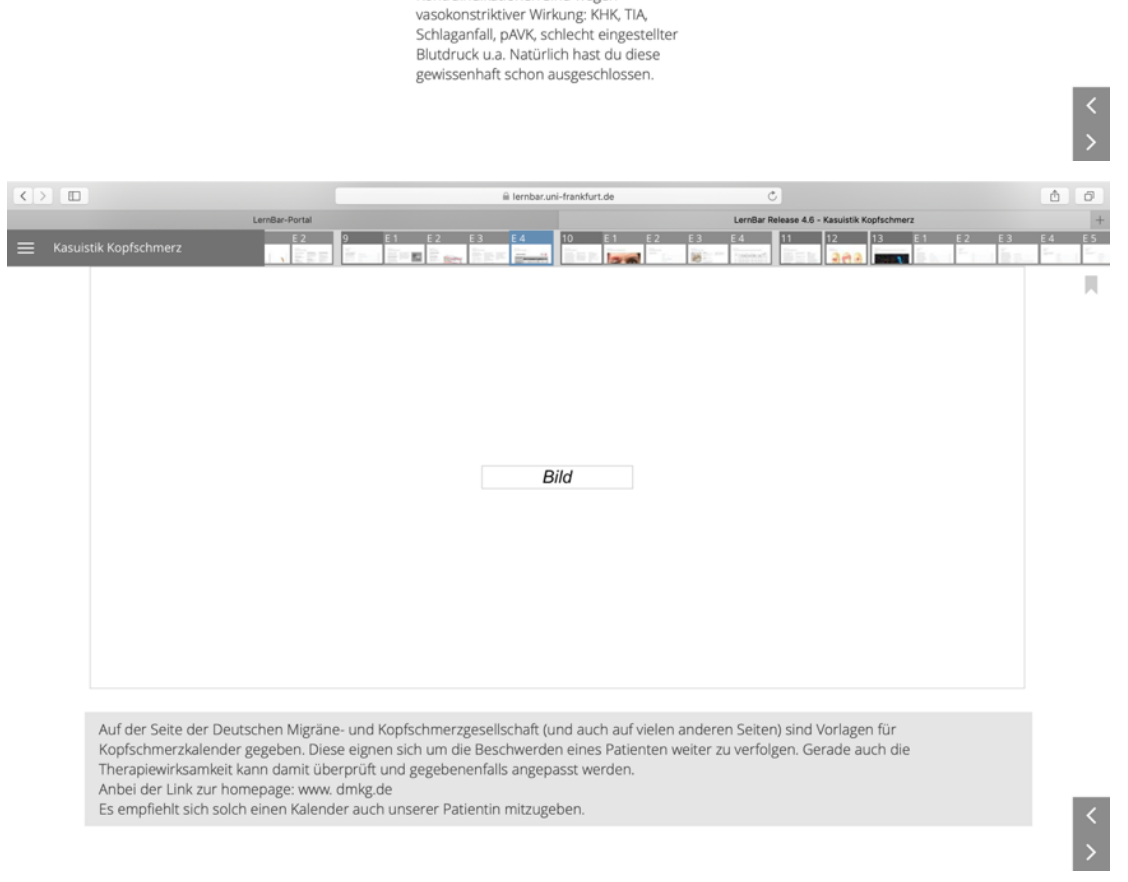




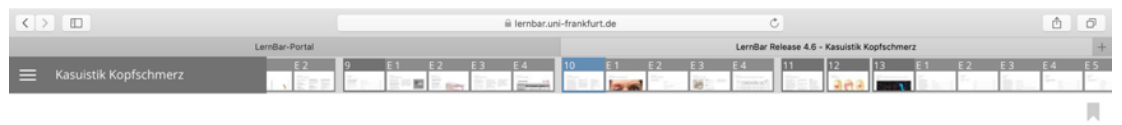

Kasuistik Kopfschmerz

Exkurs: Cluster-Kopfschmerz

Der Clusterkopfschmerz gehort zur Gruppe der trigemino-autonomen

Be dieser

primären Kopfschmerform treten - hä

nachts - stärkste und streng einseitige

Kopfschmerzattacken im Bereich des Auges

auf. Weiterhin gehören autonome

Symptome wie konjunkivivale Injektion,

Tranentuss und Miosis zum kinischen Bid.

periodische Häufung der Attacken íter

Wochen (Cluster') an die sich ein

beschwerdefreeies Intervall anschileßt. Es

werden eine episodische und eine

Die Ätiologie ist noch ungeklärt, also möglicher Triggerfaktor wird Alkoho

Attonome Symptome

Andestens eines obligat und immer

Konjunktivale Injektion und/oder

列

(n)

oft kommt es zu solchen Attacken währe

der Nacht, die Betroffenen wachen mit

acrimation (Tränenfluss)

- nkomplettes Horner-Syndrom (nur Ptosis

nd/oder Miosis)

- Schwitzen

Gesichtsrötung

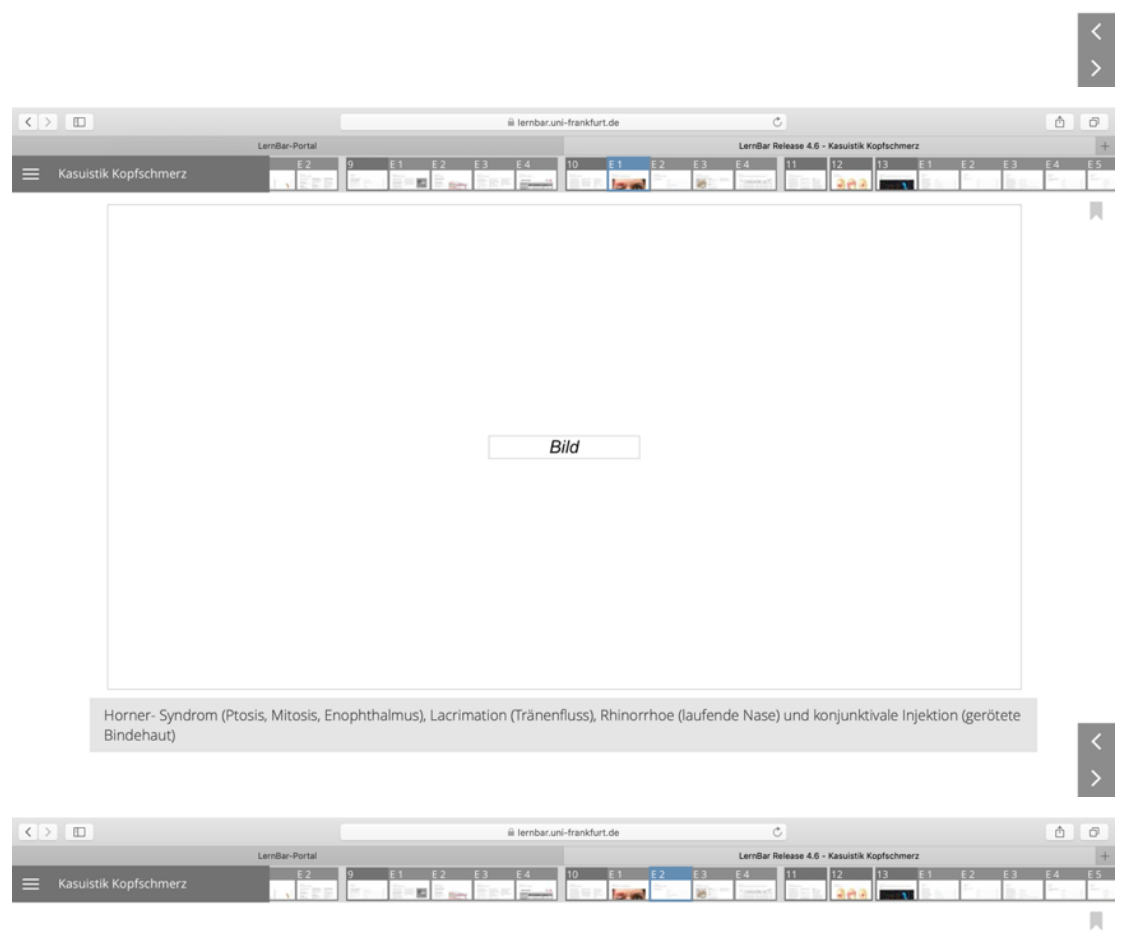

Kasuistik Kopfschmerz

Cluster-Kopschmerz Therapie

Wie therapierst du am besten eine

(atracke?

\begin{tabular}{ll} 
1. Opioidgabe i.v. & 0 \\
\hline 2. $100 \% 02$ & 0 \\
\hline 3. Betablocker oder Kalziumantagonisten & 0 \\
\hline 4. NSAR wie z.B. ASS & 0
\end{tabular}

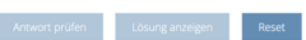




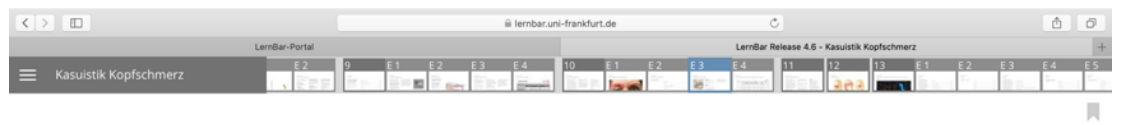

Kasuistik Kopfschmerz

Cluster-Kopfschmerz Therapie

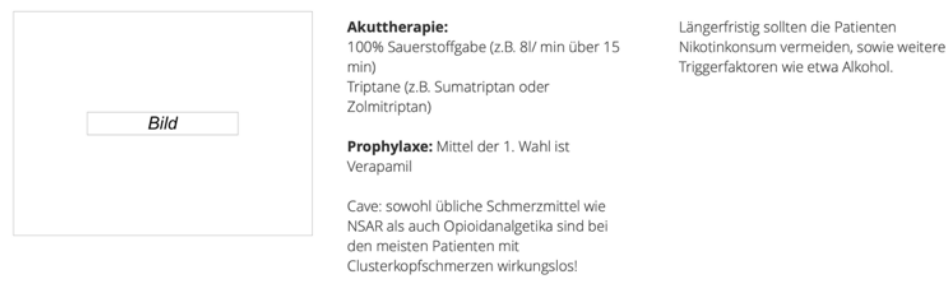

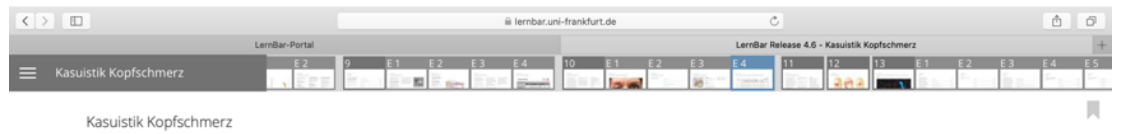

Auch zum CLuster-Kopfschmerz gibt es einen Kopfschmerzkalender

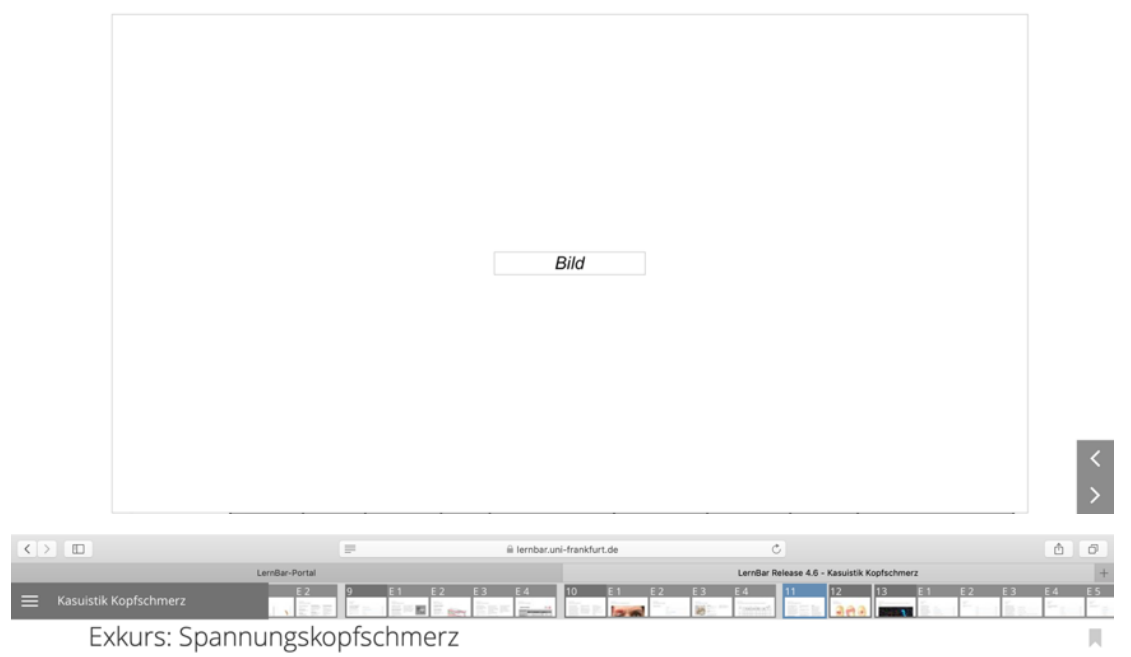

Exkurs: Spannungskopfschmerz

Beim Spannungskopfschmerz bzw. Koptschmerz vom Spannungstyp handelt es sich um den haungsten Koplschmerztyp. ist in ihren Ursachen noch unzureichens verstanden. Depressionen, Angststörungen und Stress gehören zu den ätiologischen Faktoren. Unterschieden werden der episodisch und der chronisch auftretende

Episodischer Kopfschmerz vom

Spannungstyp: Insg. mind. 10 Episoden an
durchschnittlich < 15 Tagen pro Monat über mind. 3 Monate

Chronischer Kopfschmerz vor

Spannungstyp. Koptschmerzen an

durchschmitich 215 Tager
Die Kopfschmerzen sind typischerweise pital oder holozephal okalis

Charakter: dumpf drückend, Engegefüh (.SChraubstockgefühl')

meist leichte bis mäBige Intensităt

weitere Charakteristika: keine Verstärkun durch korperliche Tatigkeit, keine

vegetatven Beglelsymptome, allenfalls

(leztere nur bei der chronischen Form)

t++ Erbrechen ist mit Kopfschmerzen vom Spannungstyp nicht vereinbar! +++

Bild
Therapie:

Die episodische Form wird akut $n$

găngigen Schmerzmitteln wie

Paracetamol behandelt. Bei der

chronischen Form sollte eine

medikamentöse Prophylaxe mit Amitriptylin

anstreben. Akut kann man einen

chronischen Spannungskoptschmerz auch

mat we die episodische form mit NSAids

benanden, jedoch darf fer patent niche

akute Analgetika. Einnahme haben Bei

beiden Formen des

Spannungskopfschmerzes sind nich-

medikamentöse Maßnnahmen wie

Entspannungstechniken oder Massagen

wesentiche Bestandtelle der Therapie. Eine

ist die Entuichtung eines Medikamenten.

induzierten Kopfschmerzes durch zu

häufige Analgetikaeinnahme.

In der Akuttherapie hilft neben ASS,

lbuprofen und Paracetamol auch das

Auftragen von Pfefferminzöl auf Stirn und

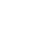




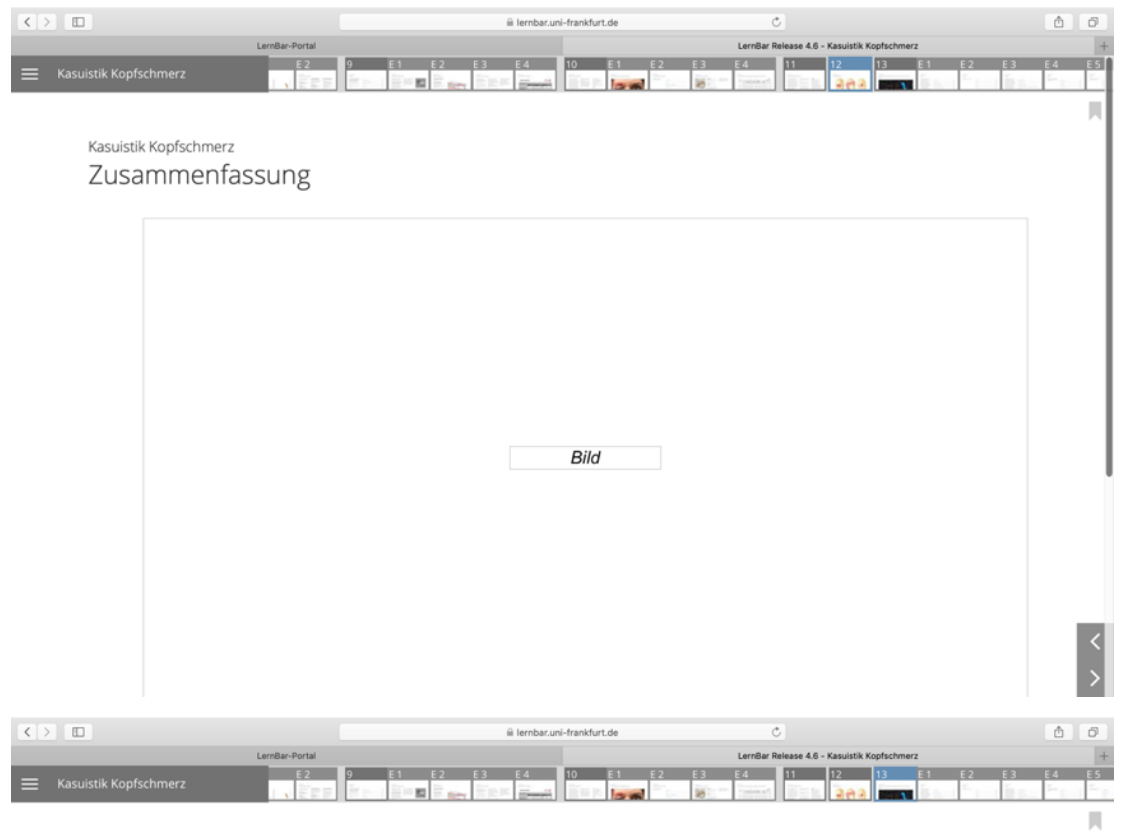

Kasuistik Kopfschmert

Super, nun ist die Kasuistik abgeschlossen. Zum Schluss noch 5 Fragen zum üben :)

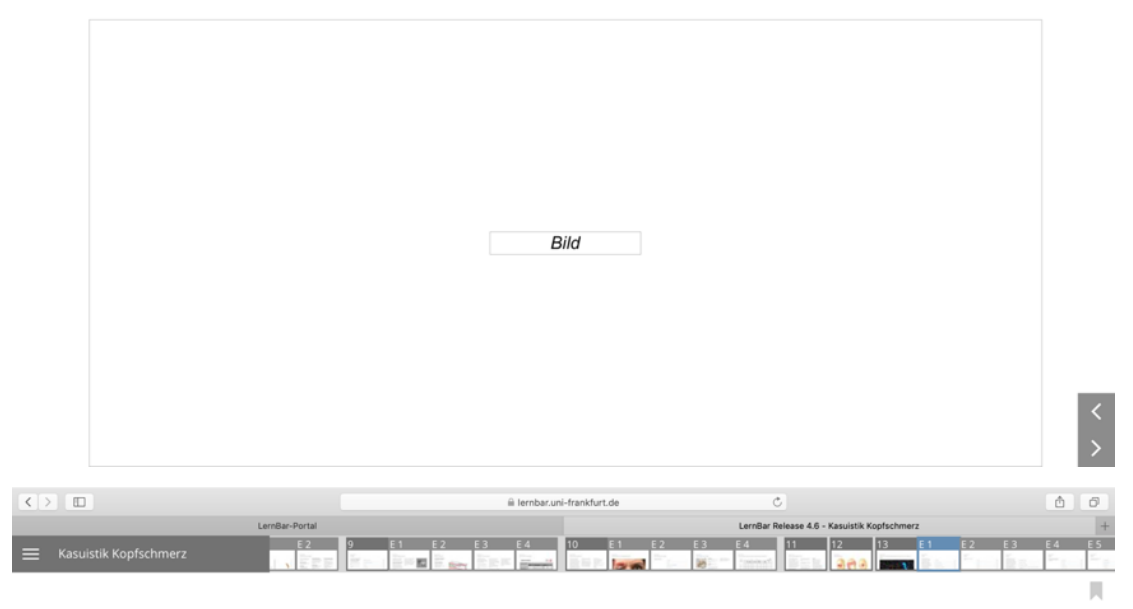

Kasuistik Kopfschmerz

Frage 1

Ein 35-jähriger Kraftfahrer und

tentrits

Kopfschmerzen af die sich artackenfor

ca. zweistündlich wiederholen und jeweils

30-60 Minuten anhalten. Typische -

rechtsseitige - Befunde während der

Attacke sind zudem konjunktivale injektion.

Lakrimation, Rhinormo und ein Horner.

Syndom wech der folgenden

\begin{tabular}{lc} 
1. O2-Inhalation & 0 \\
\hline 2. Gabe von Glyceroltrinitrat & 0 \\
\hline 3. infraorbitale Alkoholinjektion & 0 \\
\hline 4. Thermokoagulation des Ggl, stellatum & 0 \\
\hline 5. iv. Infusion von Valproat & 0
\end{tabular}

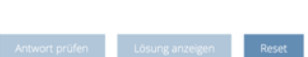




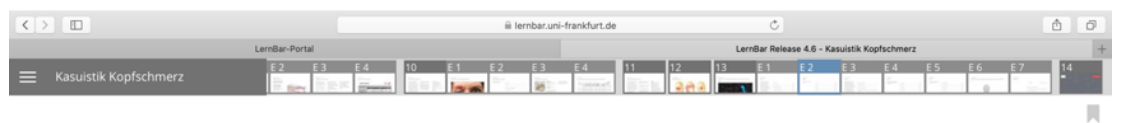

Kasuistik Kopfschmerz

Frage 2

Was ist bei Migräne am wenigsten zu

\begin{tabular}{ll} 
1. Nasenfluss & 0 \\
\hline 2. Aphasie & 0 \\
\hline 3. Skotome & 0 \\
\hline 4. Lichtscheu & 0 \\
\hline 5. Brechreiz & 0
\end{tabular}

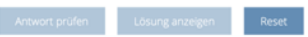

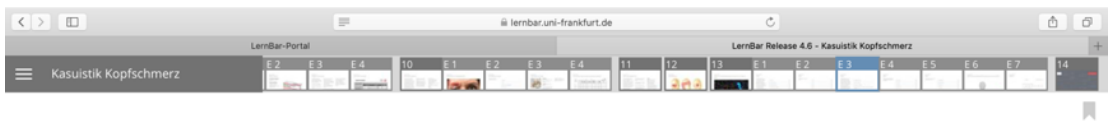

Kasuistik Kopfschmerz

Frage 3

Ein 49-jähriger Lehrer leidet seit Jahren

mines

drückenden beidseitigen Kopfsch

mit bifrontalem Schwerpunks. Es fuhres

dann etwa so an als habe er einen Helm

auf dem Kopf. Zu Ubelkeit oder Erbrechen

komme es nicht, auch konnne er trotz dieser

Deproch beenurachigen sie seine

Dennoch beentrachigten sie seine

üblichen Kopfschmerzmittel wie

Acetylsalicylsäure, Paracetamol oder

ibuprofen nicht anschlügen. Er rauche ca.

20 Zigaretten pro Tag, schlafe vermutlich

stressbedingt schlecht, sei sonst aber

gesund. Der kinischneurologische Belund

ist ink. einer Augenhintergrundspiegelung

folgenden medikamentösen Therapien

kommt für diese Kopfschmerzen am

ehesten in Betracht?

\begin{tabular}{lc} 
1. Acetylsalicylsäure $100 \mathrm{mg} / \mathrm{d}$ & $\bigcirc$ \\
\hline 2. Amitriptylin $50-150 \mathrm{mg} / \mathrm{d}$ & 0 \\
\hline 3. Carbamazepin $400-1600 \mathrm{mg} / \mathrm{d}$ & 0 \\
\hline $\begin{array}{l}\text { 4. Glukokortikoid-Therapie oral (z.B. Prednisolon-Schema beginnend mit } 1 \mathrm{mg} / \mathrm{kg} \\
\text { Körpergewicht) }\end{array}$ & $\bigcirc$ \\
\hline 5. Indometacin $50-150 \mathrm{mg} / \mathrm{d}$ & $\mathrm{O}$
\end{tabular}

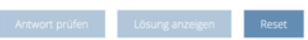

(1) 四

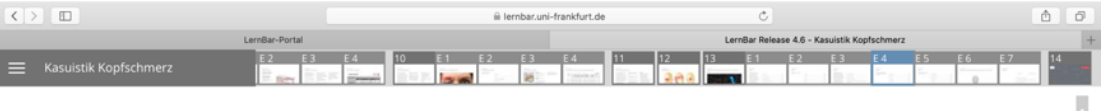

Kasuistik Kopfschmerz

Frage 4

Welches der folgenden Medikamente ist für geeignet?

\begin{tabular}{lc} 
1. ASS $1000 \mathrm{mg}$ i.v. & 0 \\
\hline 2. MCP $20 \mathrm{mg}$ i.v. & 0 \\
\hline 3. Sumatriptan i.n. & 0 \\
\hline 4. Propanolol $75 \mathrm{mg}$ p.o. & 0 \\
\hline 5. Ibuprofen $600 \mathrm{mg}$ i.v. & 0
\end{tabular}

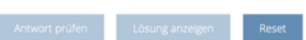




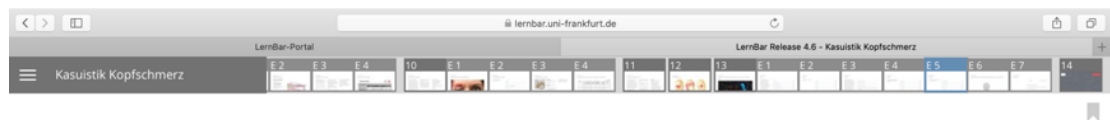

Kasuistik Kopfschmerz

Frage 5

Welche Symptome können nicht zur Aura einer Migräne gezählt werden?

\begin{tabular}{lc} 
1. Gesichtsfeldausfalle & 0 \\
\hline 2. Konjunktivale Injektion & 0 \\
\hline 3. Flimmerskotome & 0 \\
\hline 4. Szintillationen & 0 \\
\hline 5. Ophthalmoplegie & 0
\end{tabular}

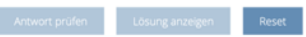

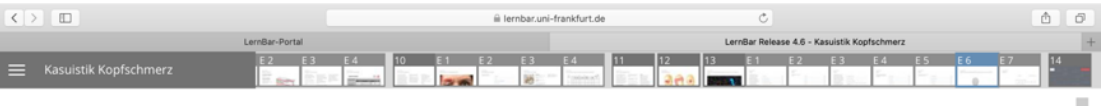

Kasuistik Kopfschmerz

Super! Du hast die Kasuistik geschafft! Bitte evaluiere noch kurz die Kasuistik!

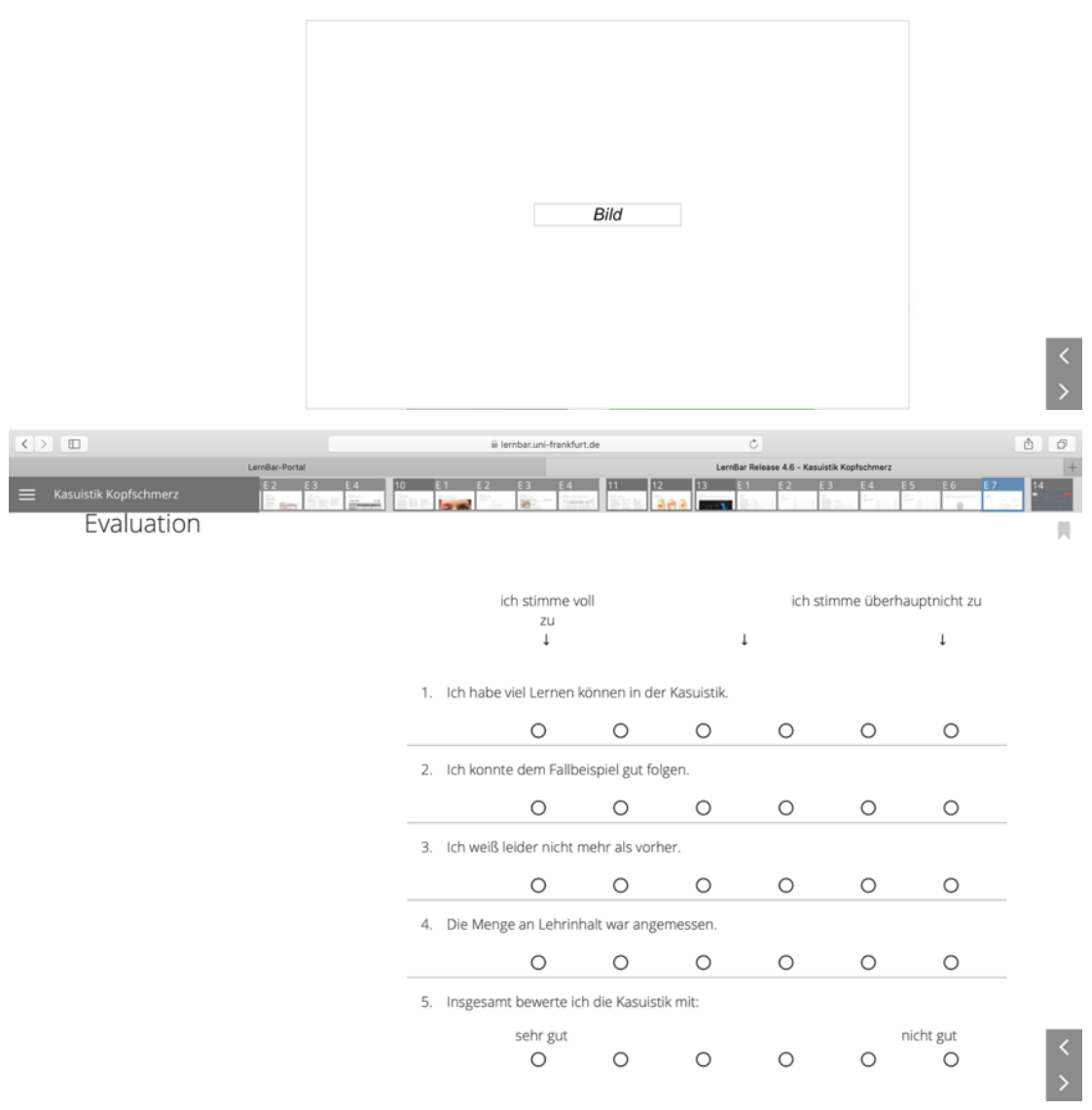




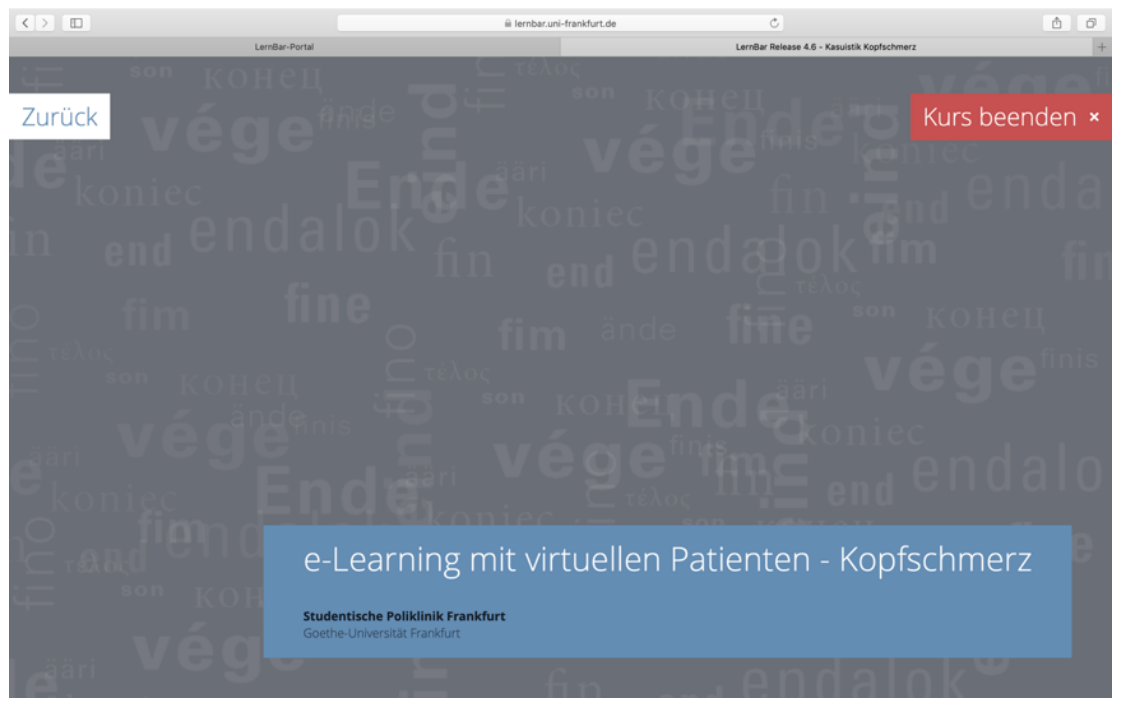


Kasuistik Kreuzschmerz

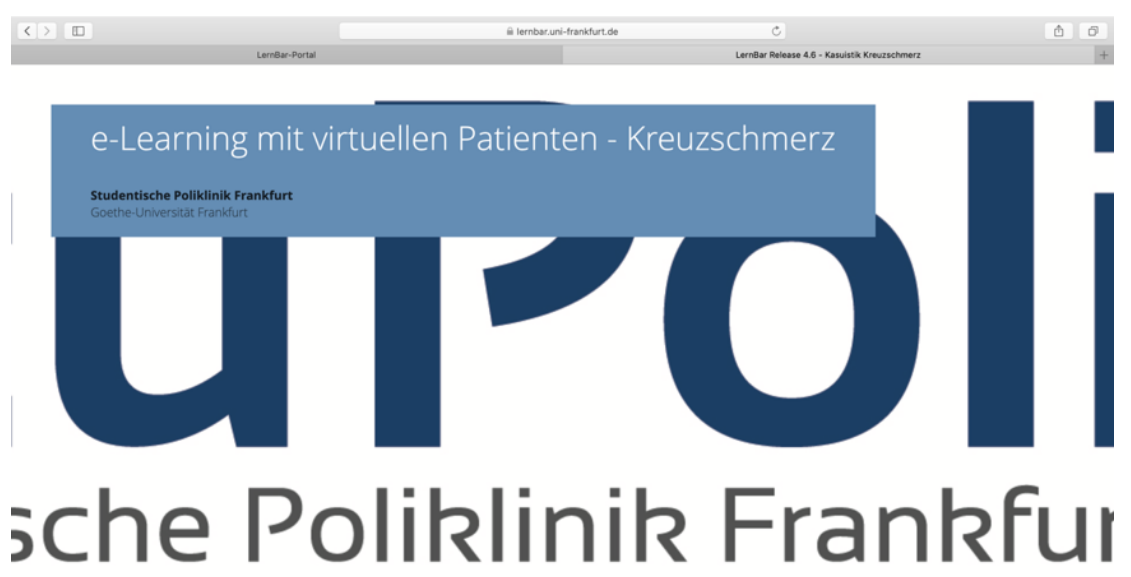

\section{Goethe Universität KURS STARTEN}

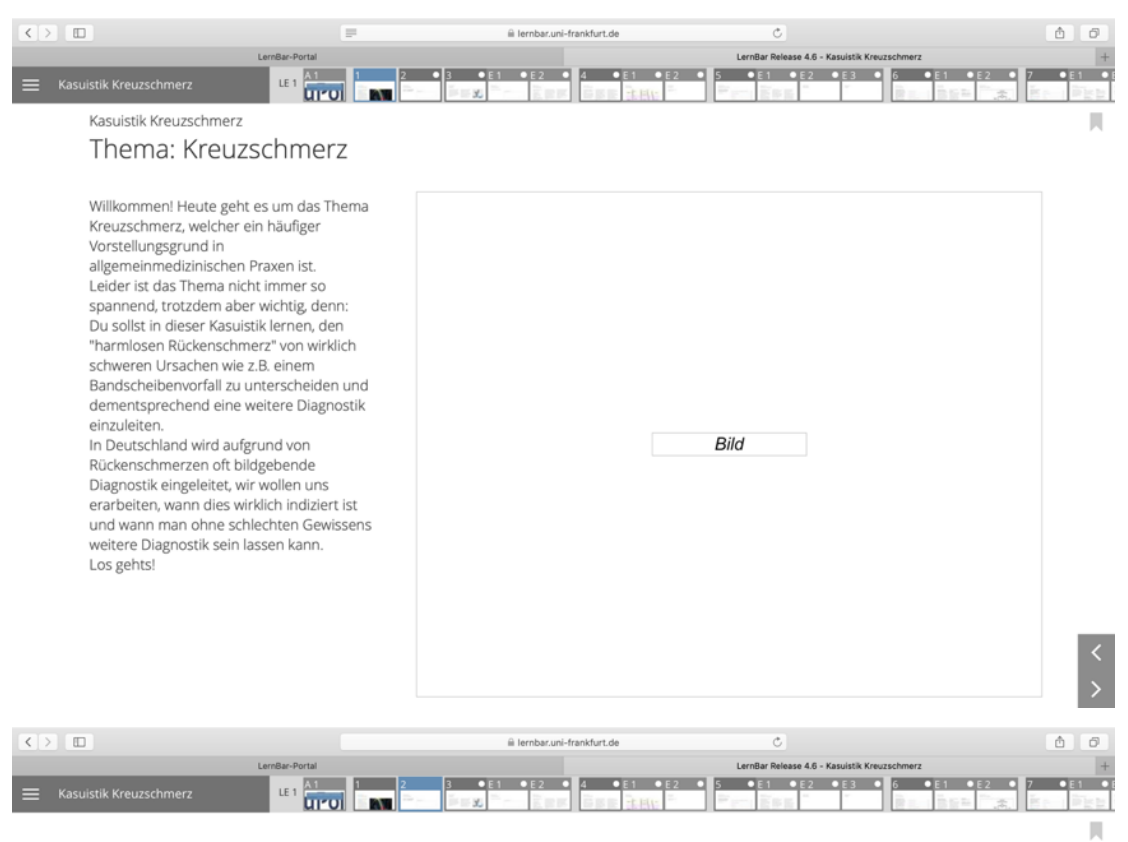

Kasuistik Kreuzschmerz

Persönliche Daten

Bitte schreibe in das Feld deinen Vor-und

Namen und deine Matrikelnummer.

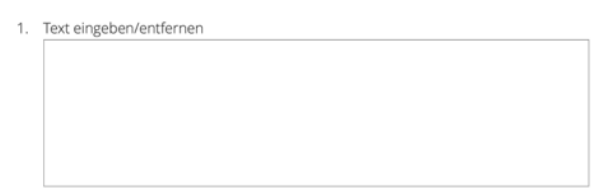




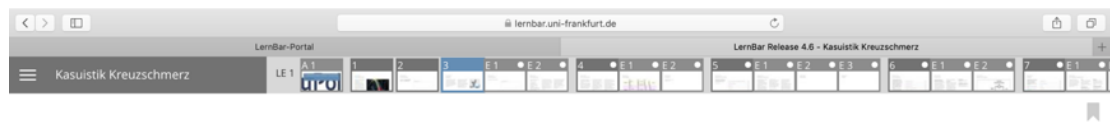

Kasuistik Kreuzschmerz

Fallbeispiel

Herr Manfred Keller, 60 Jahre, kommt zu Sprechstunde. Du schaust in seine Akte Infekts da war. Seine Frau ist ebenfalls bei dir in Behandlung bei ihr kontrollierst du regelmäßig den Blutdruck, da sie

Herr keller berichtet, dass er seit scho habe langerer zet Ruckenschmerzen Daher sei er nun zu dir gekommen er weis nicht was er dagegen tun kann, das Aspirn. das er genommen hat, hătte ihm nicht

geholfen.

Du fragst genauer nach um dir ein Bild

rer Beschwerden au machen.
Bild

(2)

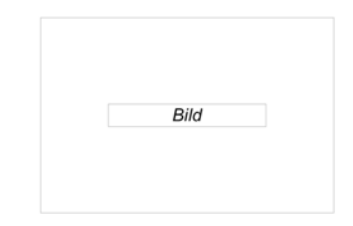




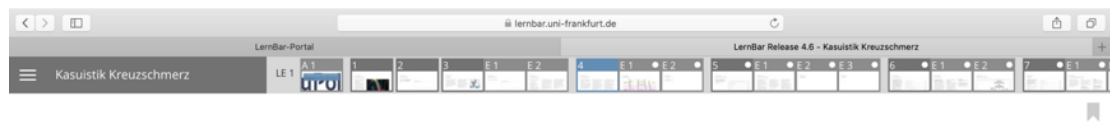

Kasuistik Kreuzschmerz

Exkurs in die Vorklinik

Ja... ist nicht grad ein Highlight des

(

kurze und relevante Wiederholung zur

Die Wirbelsäule bildet das Achsenskelett

des menschlichen Korpers und garantient

Stabilităt bei sehr hoher Beweglichkeit.

Aufgrund inrer wichtigen Funktionen ist de

Wirbelsaule alterdings auch von vielen

Bis zum 50. Lebensjahr haben mehr als

Wirbelsäulenerkrankungen füh

Arbeitsausfälle in Deu

Diagnose "Rückenschmerz" ist fuhrend in

en Mordictasstatsiken

Aitag ist es deshalb besonders wichtig

können.
(Quelle: Amboss)
Außer dem Wissen über den anatomischen

Auter

zu kennen. Falls du den

Verdacht auf einen Bandscheibenvorfall

hast, kannst du so zuordnen in welchem

Segment der Vorfall stattgefunden haben

muss. Dabei ordnest du einfach der

Symptomatik (z.B. Hypästhesien,

Kraftgradminderungen) das passende

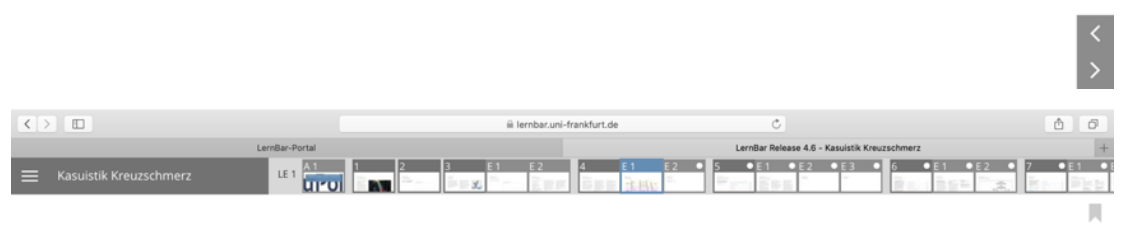

Kasuistik Kreuzschmerz

Die Wirbelsäule

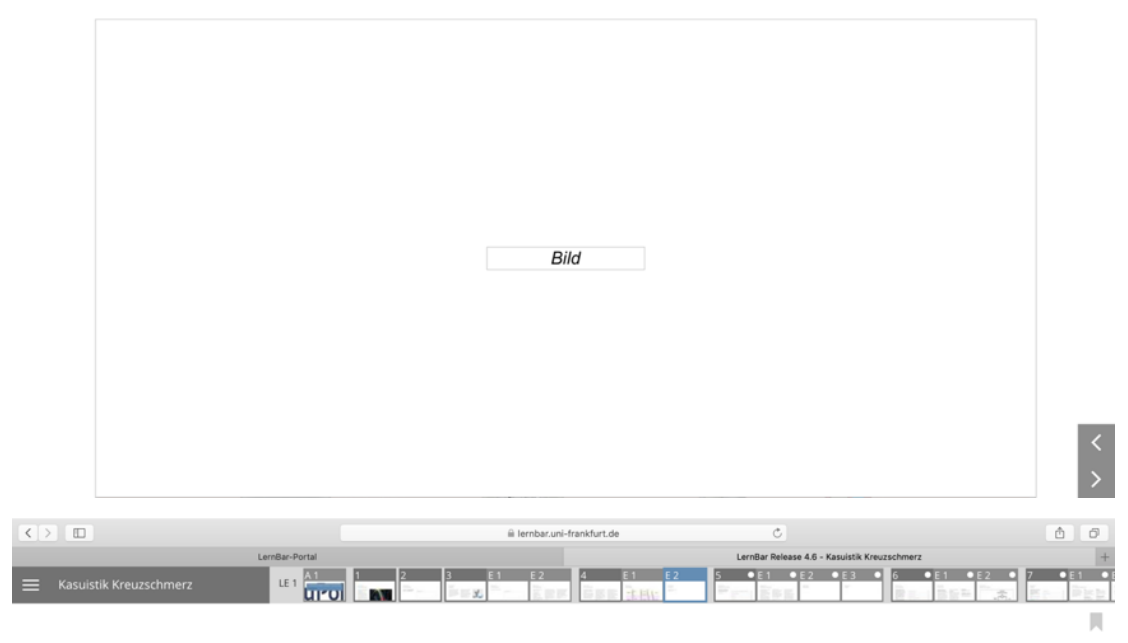

Kasuistik Kreuzschmerz

Dermatome

Hier siehst du noch einmal, wie die

Bild 


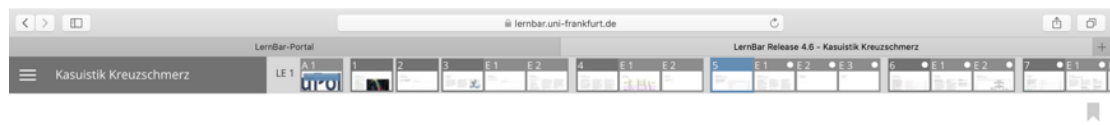

Kasuistik Kreuzschmerz

Körperliche Untersuchung

Neben Blutdruck, Puls und allgemeinen

du eine spezifische Untersuchung.

\begin{tabular}{lc} 
1. Inspektion von Gangbild, Wirbelsäule und allgemeiner Körperhaltung & $\square$ \\
\hline 2. Klopfschmerz der Wirbelsäule & $\square$ \\
\hline 3. Babinski- Reflex & $\square$ \\
\hline 4. Palm- up- Test & $\square$ \\
\hline 5. Schober und Ott Test & $\square$
\end{tabular}

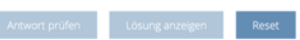

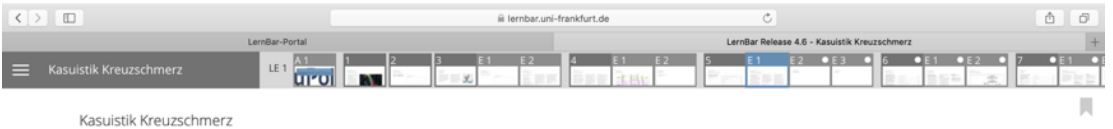

Körperliche Untersuchung

Richtig sind: Inspektion von von Gangbild,

Wirbelsăule und allgemeiner

Da der Patient anfangs von ausstrahlende Schmerzen benichtet hat, fragst du genaue

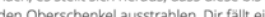
dass du die Sensibilitatt überpriüen kann und tust dies Dermatom- orientiert. Es

Wirbelsăule; (Babinski- Reflex), schooberRahmen der Funktionsüberprüfung des

Schultergelenks statt.

inden sich keine Sensibilitätrsstorungen

oder motorischen Ausfalle.

Du kannst Herrn Keiler einmal auf

abgehen lassen um das Gangbild

(Symmetrie, Anken etc.) zu beurteilen. Die

Kyphose und L lordose Rippenbuckel und

Du kannst nun auch gezielt Tests

durchführen.

lasst, misst du den Finger- Boden- Abstand

der in unserem Fall $15 \mathrm{~cm}$ ist.

Es gibt 2 Tests, die du können solltest:

en Schluter- und Beckenstand

Schober- - und Ott- Test.

(Musketverspannung?). Dann klopfst du

zunächst leicht dann mit twas Druck (aber

trotzdem vorsichtig) die Wirbelsăule von

kranial nach kaudal ab.

unauffallig jedoch findet sichein

umschriebener diskreter Klopfschmerz der

lumbalen Wirbelsăule vor.

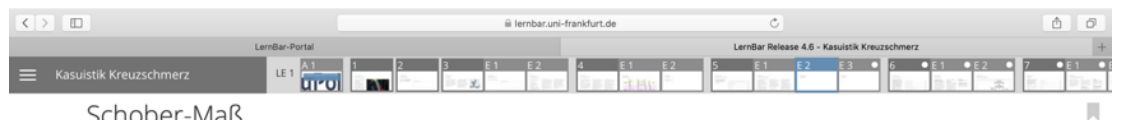

Schober-Maß

Mit dem Lasègue- Zeichen kannst d

uberprufen, ob der Patient einen

Spinalnervenwurzeln der

Rückenmarkssegmente L4.S2 (oder N.

ischiadicus) hat. Dafür legt sich der Patient

auf den Rücken und du führst eine

Beugung im Hưftgelenk durch. Sollte es zu

Schmerzen kommen, dann ist das Lasègue

Zeichen positu und konnte 2.B. Hinwes a

Bei Herrn Keller ist die Hüfte zwar nich

mehr so gut beweglich, jedoch hat er keine

Schmerzen und somit ein negatives

Lasègue-Zeichen!

Hier kannst du dir noch mal ein Video (www) anschauen um die Untersuchur

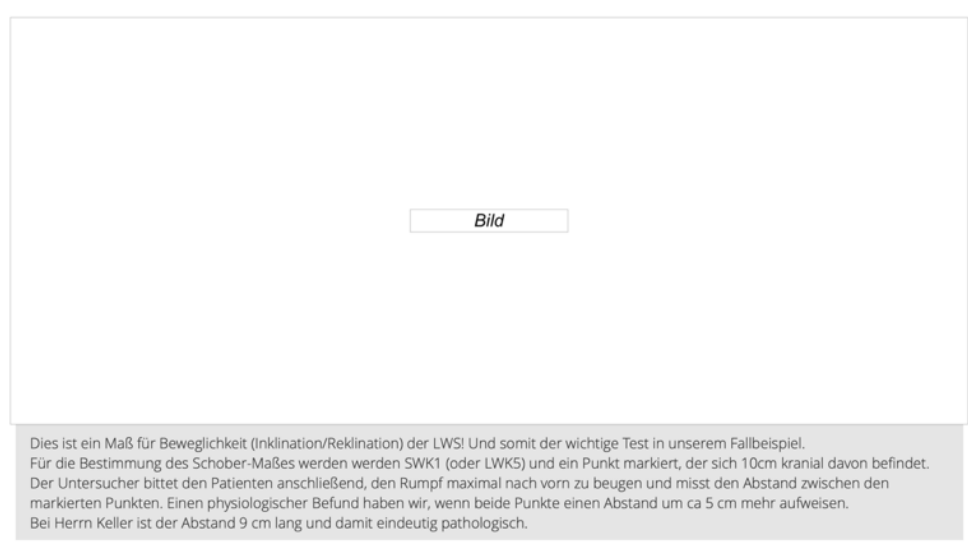



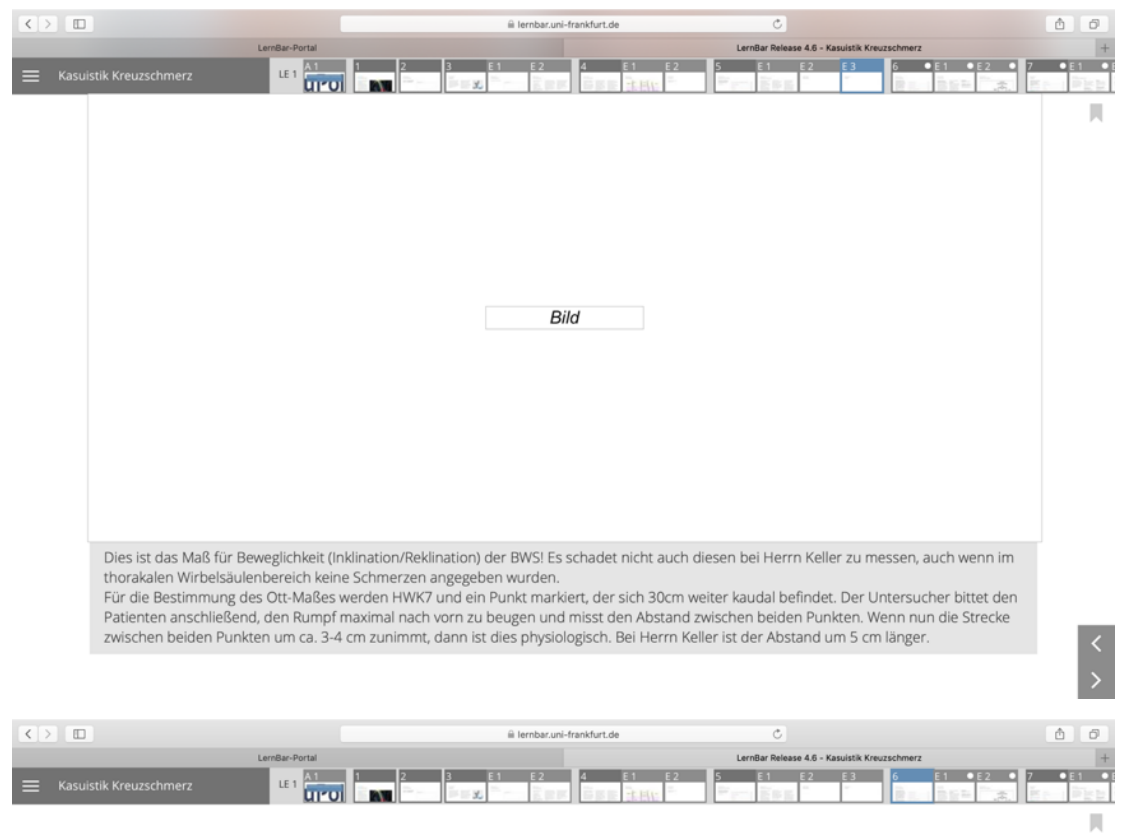

Kasuistik Kreuzschmerz

Zusammengefasst...

Manfred Keller hat seit 4.5 Wochen

bewegungsabnangige Schmerzen im

den Oberschenkel. Es ist ein diskreter

Klopfschmerz an besagter Stelle

vorhanden. Der Finger- Boden- Abstand

ist bei $15 \mathrm{~cm}$ pathologisch und das

Schober-Maßs ebenso. Es finden sich

keine sensiblen oder motorischen

... Du denkst darüber nach wie du die

Was ist das für eine Art von Kreuzschmerz?

\begin{tabular}{lc} 
1. Unkomplizierter Kreuzschmerz & 0 \\
\hline 2. Radikulärer Kreuzschmerz & 0 \\
\hline 3. Komplizierter Kreuzschmerz & 0 \\
\hline 4. Extraradikulärer/-vertebraler Kreuzschmerz & 0 \\
\hline 5. Kreuzschmerz unklarer Genese & 0
\end{tabular}

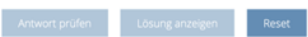

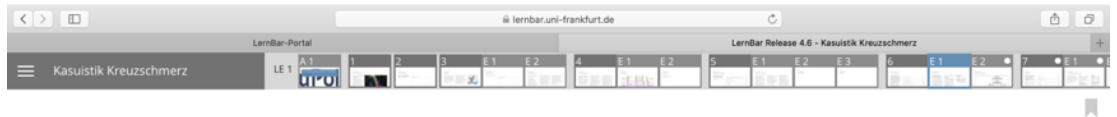

Kasuistik Kreuzschmerz

Kreuzschmerz

Super, die richtige Antwort ist:

merz, welchen

man auch als rexench

Kreuzschmerzes, welche dir in der

hausärztlichen Praxis begegnen wird.

Dieser Rückenschmerz tritt meist spontan

oder nach körperlicher Beanspruchung au,

sie konnen sich je nach korperthatung oder

Bewegungsablaut ander Auch strahien

beide) aus, wobei hier meist nur der

Oberschenkel betroffen ist. Die Schmer

strahlen nicht über das Knie aus (im

Gegensatz zu einem Bandscheibenvorfa

beispielsweise).
Weitere Formen von Kreuzschmerz

\section{Radikulärer KS:}

Dieser entsteht durch Einklemmung einer schialgie Oft tritt diese in Zusammenhar

mit einem Bandscheibenvorfall auf.

Differentialdiagnostisch strahlen die

Schmerzen haufig uber das Knie hinaus

aus tort bis zum grosen Zeh oder Fußs,

Ausfalle können Beglettsymptome sein.

sowie Reflexabschwächung- bzw.

sowie Reflexa
Pathologien.
Komplizierter KS:

ther solltest du an Red Flags denken daz

gleich mehr!

Extraradikulärer/- vertebraler KS:

Die Schmerzen kommen also nicht von der

Wirbelsäule selbst, sondern von

umgebenden Organen/ Organsystemen 


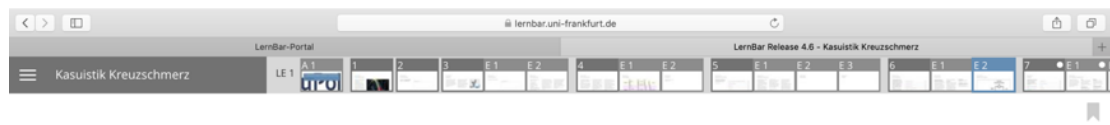

Kasuistik Kreuzschmerz

Häufiges ist eben häufig ...

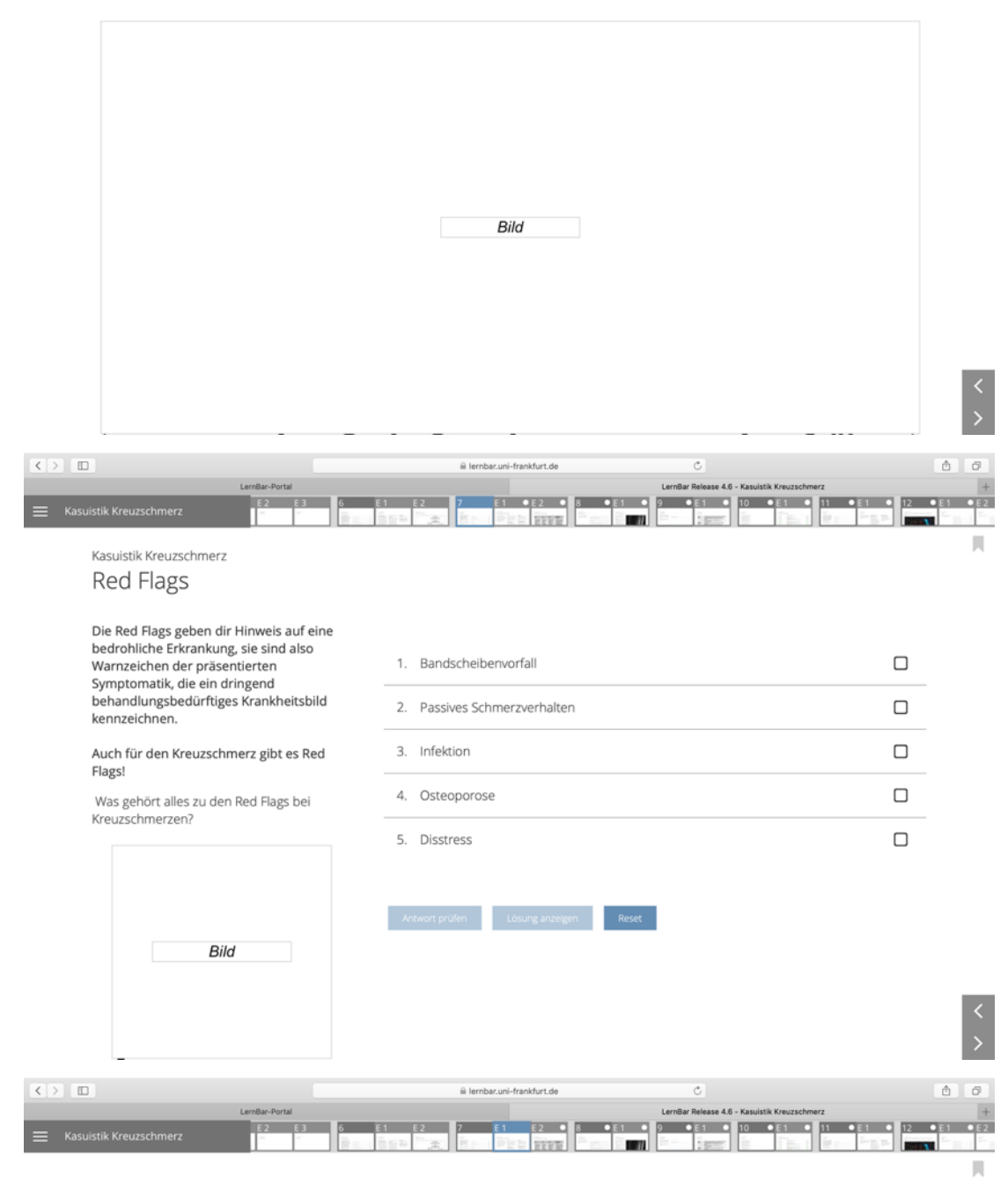

Kasuistik Kreuzschmerz

Die Red Flags bei Kreuzschmerzen

Es gibt 4 gefährliche Ursachen für

kreuzschmerz. Durch eine grundiche

solttest du herausfonden $\mathrm{kounnen}$.

davon Ursache für die Schmerzen sein

könnte. Falls die Symptome dafür

sprechen, ist in jedem Fall eine weitere

Thochentumor, Knochenmetastasen)

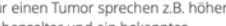

Tumorleiden Du solltest nach

Symptomatik fragen, was dir einen Hinweis

geben kann.

Wirbelkörperfrakturen:

Diese können durch ein Trauma entstehen

in höherem Alter oder aber auch bei z.B.

durch Bagatelltraumata entstehen.

Infektionen:

Fieber, Schüttelfrost im Rahmen von

Aber natürlich auch ein bekannter

Infektfokus oder Immunsuppression sind

abzufragen.

Retikulopathien/ Neuropathi

Ort strahlien die Schmerzen aus, je nach

sein. 


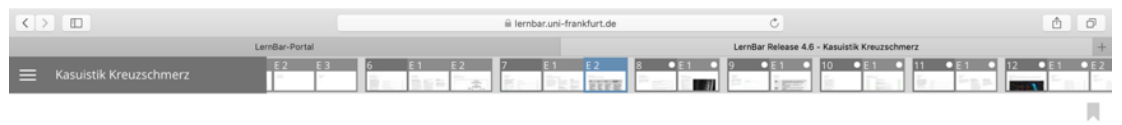

Kasuistik Kreuzschme

Die 4 Red Flags bei Kreuzschmerzen

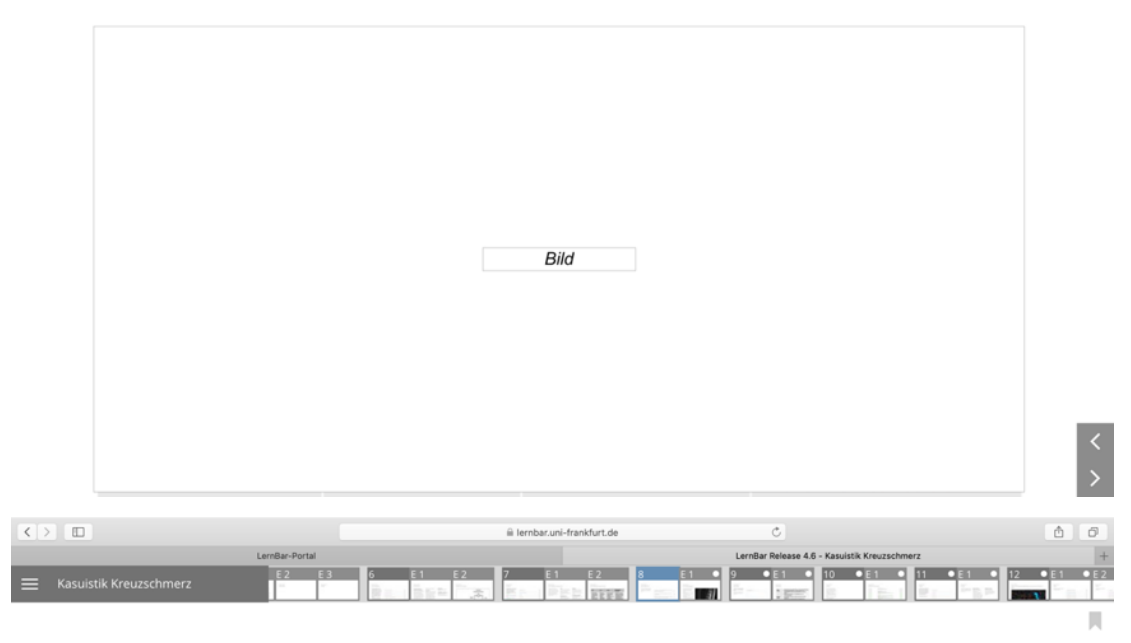

Kasuistik Kreuzschmerz

Der nächste Schritt?

Wir haben Hern Keller gut untersucht und

keinentrelevanten Hinweis auf eine Red

Flag gefunde

\begin{tabular}{ll}
$\begin{array}{l}\text { 1. Ich überlege, dass eine bildgebende Diagnostik genau das richtige wäre um } \\
\text { gefáhrliche Ursachen auszuschließen. }\end{array}$ & 0 \\
\hline $\begin{array}{l}\text { 2. Ich verschreibe ihm nur Physiotherapie und bestelle ihn in } 2 \text { Wochen zur } \\
\text { Verlaufskontrolle ein. }\end{array}$ & 0 \\
\hline $\begin{array}{l}\text { 3. Ich überlege, welche Faktoren bei Herrn Keller zur Schmerz- Chronifizierung } \\
\text { führen könnten. Diese Ursachen könnte man behandeli. }\end{array}$ & 0 \\
\hline $\begin{array}{l}\text { 4. Ich erteile ithm Bettruhe und versuche die sogenannten Yellow Flags } \\
\text { durchzugehen. }\end{array}$ & 0 \\
\hline $\begin{array}{l}\text { 5. Ich überweise den Patienten an einen Neurologen, da ich denke, dass die } \\
\text { Schmerzen eher ein Indiz für eine komplexe neurologische Erkrankung sind. }\end{array}$ & 0
\end{tabular}

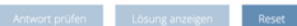

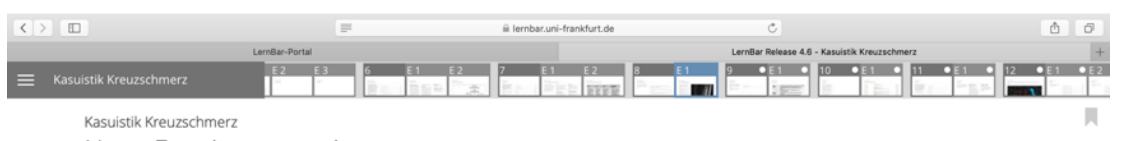

Neue Erweiterungsseite

Wenn du den Verdacht auf eine der Red

Flags haben solltest, dann ist eine

indiziert!

Etwas anders ist es, wenn du die Red Flags

ausgeschlossen hast.

en ( 66 Wochen) bieten

keine Indikation für bildgebende

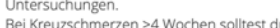

psychosozialer Risikofaktoren für eine

Chronifizierung (sogenannte "yellow flags" )

erfassen. Dazu gleich mehr...

Subakute Kreuzschmerzen (6-12 Wochen)

erfordern

uzschmerzen $>12$

Bildgebung (sofern

nicht schon zuvor erfolgt) indiziert
Mit diesem "schema" kannst du

entscheiden, wann du denkst, dass ein

MRT, Röntgen oder $C T$ gemacht werden

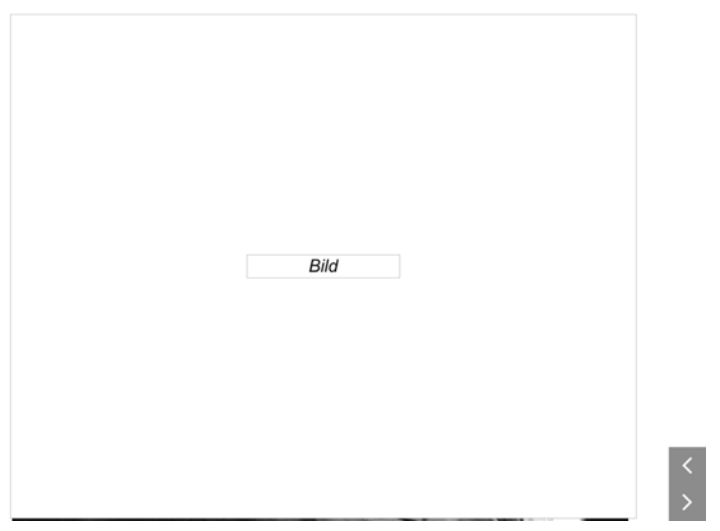




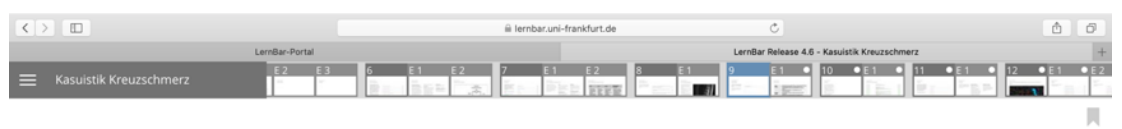

Kasuistik Kreuzschmer

Yellow Flags

"Yellow Flags" umfassen psychosoziale

Faktoren

Was denkst du zählt zu den Yellow Flags

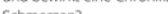
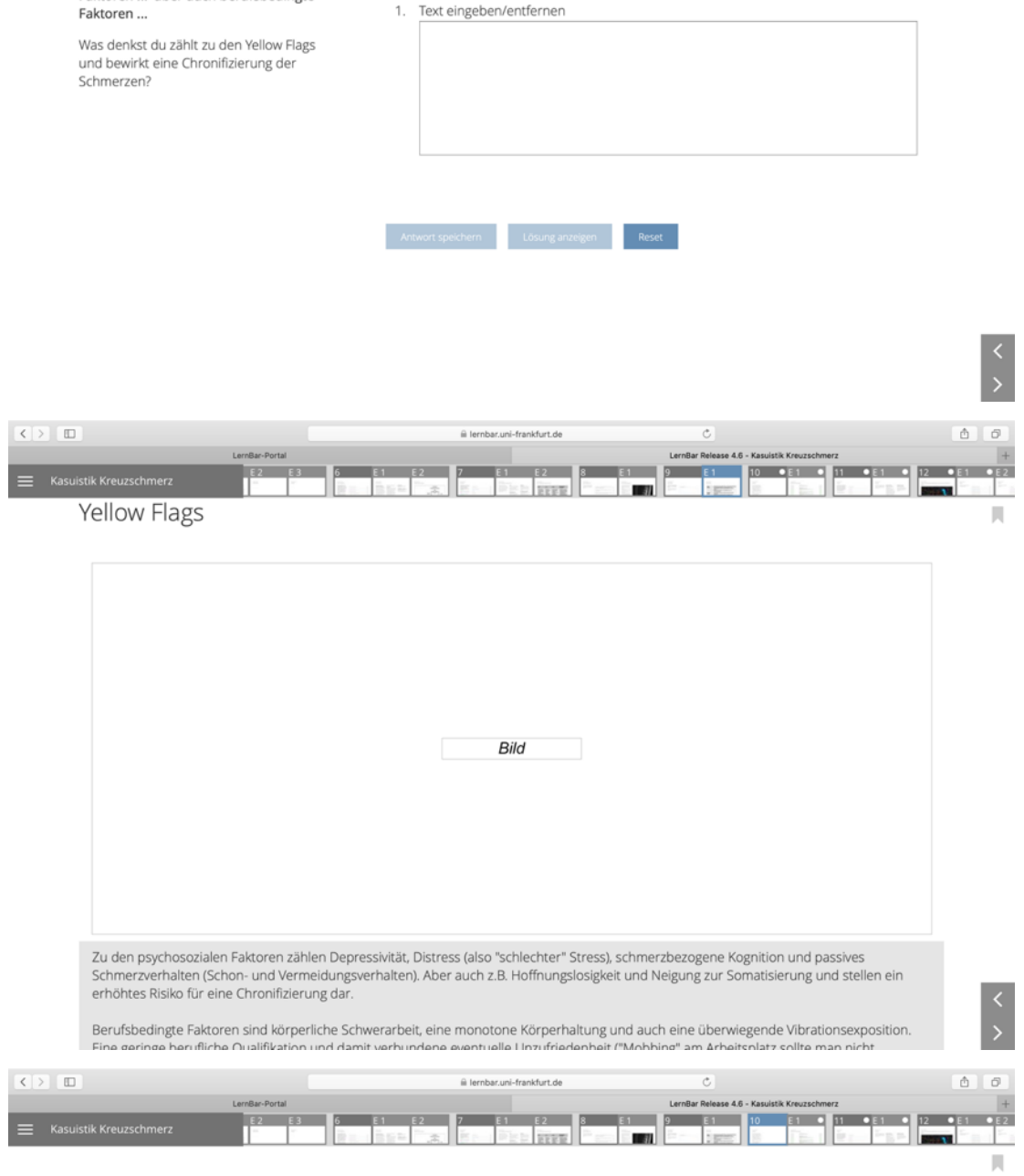

Kasuistik Kreuzschmerz

Leitlinien

Du solltest natürlich die Red Flags im

Hinterkopf haben, jedoch ist es hilfreich

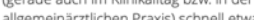

nachzuschlagen. Unser Tipp um

wo du das diagnostische Vorgehen zu

relevanten Krankheitsbildern raussuchen

kannst.

Bild

Stand und Nachschlagewerk für

Studentinnen und Ärztinnen

Hiler der Link (Mww) zur

Wissenschaftlichen Medizinischen

Fachgesellschaften (AWMF)

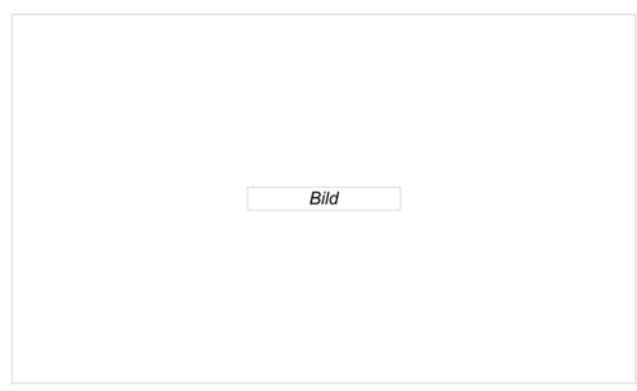




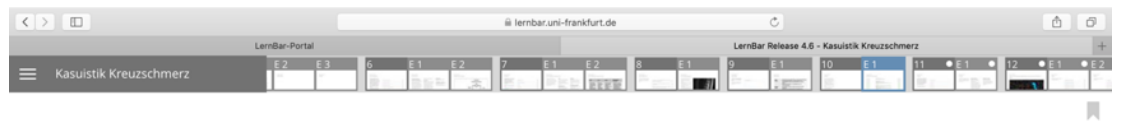

Kasuistik Kreuzschmerz

Leitlinien Kreuzschmerzen

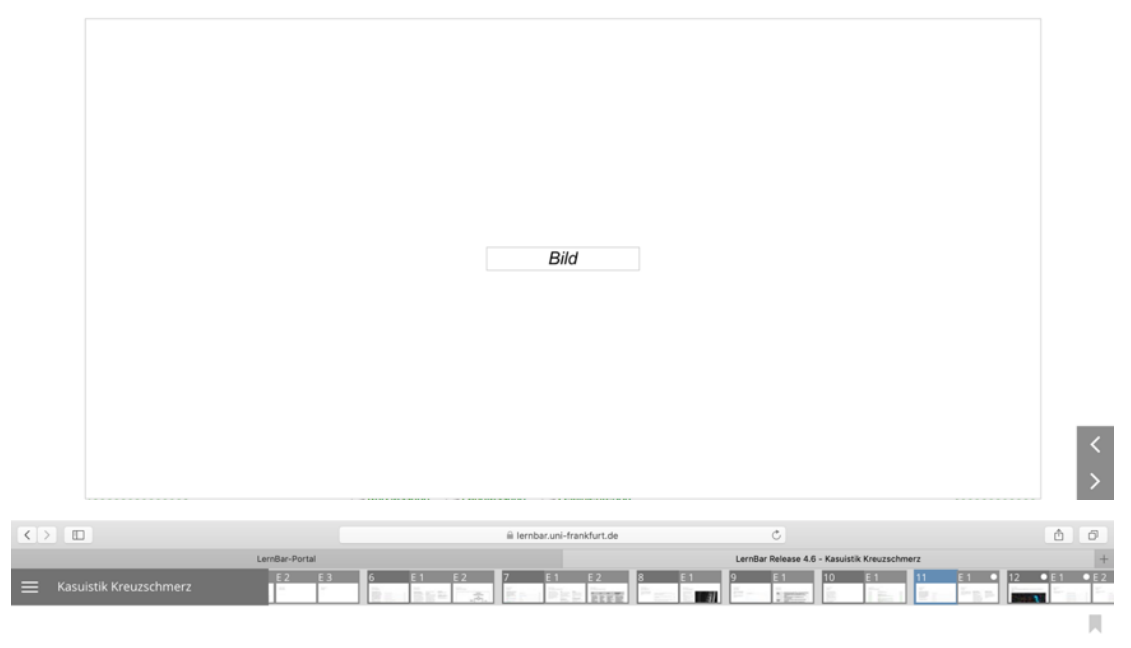

Kasuistik Kreuzschmerz

Therapie

Nun überlegst du wie du Herrn Keller

helfen kannst. Zunächst erkearsst du him,

und du ihm lieber ein anderes Mittel -

verordnest. Doch welche Schmerzmitte

dann?... Und bleibt es nur bei einer

medikamentösen Therapie?

Welches Medikament schreibst du ihm auf?

Was könnte hier stimmen?

\begin{tabular}{ll} 
1. Diclofenac $100 \mathrm{mg}$ & 0 \\
\hline 2. Ibuprofen $3 \mathrm{~g}$ & 0 \\
\hline 3. Oxycodon $4 \mathrm{mg}$ & 0 \\
\hline 4. Loperamid $5 \mathrm{mg}$ & 0 \\
\hline 5. Paracetamol $5 \mathrm{~g}$ & 0
\end{tabular}

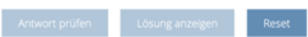

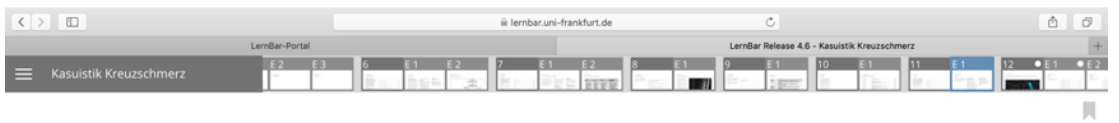

Kasuistik Kreuzschmerz

Therapie

Richtig! Diclofenac $100 \mathrm{mg}$ ist die richtige Wath siene zur medikamentosen Therape

Tonservative Therapie:

körperlicher Aktivität

unten, welche Schmerzmittel du

keine Bettruhe und keine aktive

enach psychosozialen Risikofaktoren kan

eine individuelle kognitive

Verhaltenstherapie angeboten werden.

Dir sollte in jedem Fall klar sein, dass du

nicht nur die Symptome von Herm Keler

möchtest, sondern so gut wie möglich auch

praventiv an den "Ouellen" anzugetzen.

Also z.B. Stressreduktion, verbesserte

Arbeitsverhältnisse, Behandlung von

Depression etc.

Medikamentöse Therapie:

Hier kannst du Paracetamol und

Paracetamol mit maximaler Tagesdosis von

g. Ibuprofen mit $1.2 \mathrm{~g}$ oder Diclofenac

$100 \mathrm{mg}$ sind ebenfalls geeignet und

evidenzbasiert. 


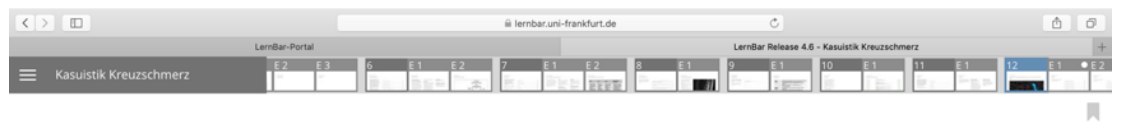

Kasuistik Kreuzschmerz

Super, nun ist die Kasuistik abgeschlossen. Zum Schluss noch 5 Fragen zum üben :)

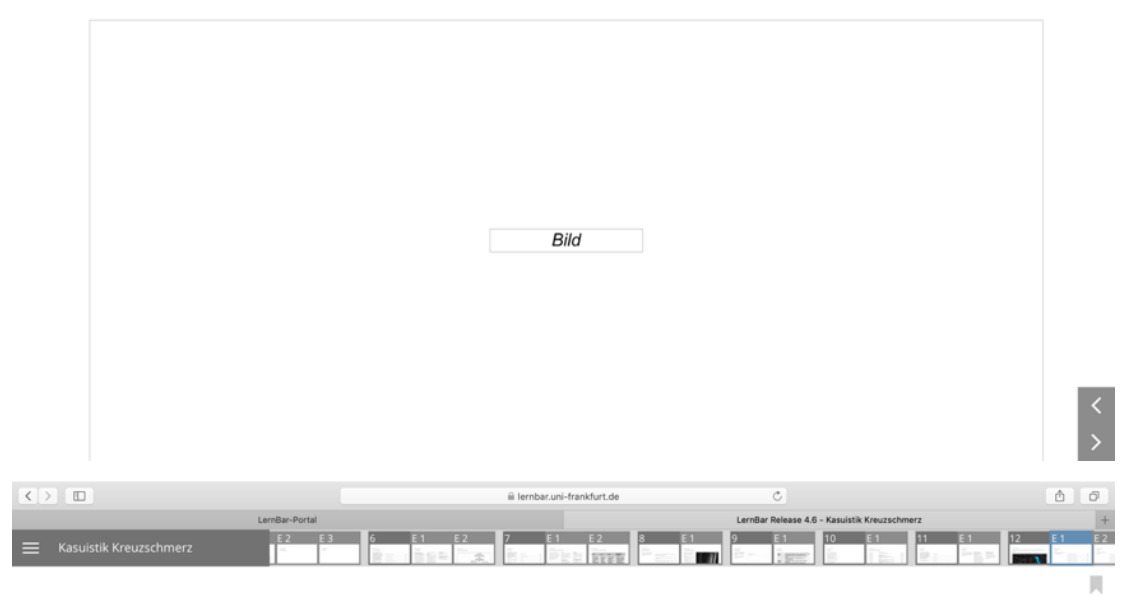

Kasuistik Kreuzschmerz

Frage 1

Das Ott-MaB (z.B. Ott 30/34 cm) dient am

\begin{tabular}{lc} 
1. Beugefăhigkeit der Brustwirbelsäule & 0 \\
\hline 2. Streckfăhigkeit der Brustwirbelsāule & 0 \\
\hline 3. Beugefăhigkeit der Lendenwirbelsäule & 0 \\
\hline 4. Streckăhigkeit der Lendenwirbelsäule & 0 \\
\hline 5. Streckfăhigkeit der Halswirbelsäule & 0
\end{tabular}

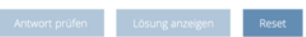

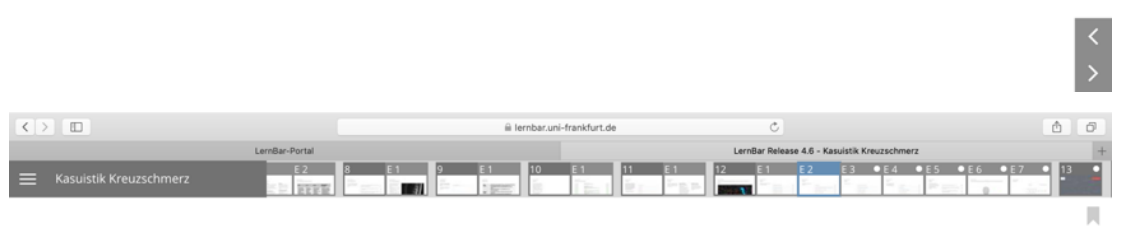

Kasuistik Kreuzschmerz

Frage 2

Was gehört nicht zu den Red Flags des Kreuzschmerzes?

\begin{tabular}{lc} 
1. B- Symptomatik & 0 \\
\hline 2. osteoporotische Wirbelkörperfraktur bei systemischer Steroidtherapie & 0 \\
\hline 3. Sensibilitätsstörungen der unteren Extremität & 0 \\
\hline 4. Blasen- und Mastdarmstörungen & 0 \\
\hline 5. Schlafstörungen & 0 \\
& \\
\hline
\end{tabular}




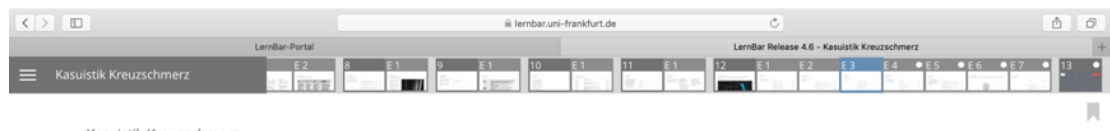

Kasuistik Kreuzschmerz

Frage 3

Was siehst du hier im Bild?

\begin{tabular}{|l|l|}
\hline 1. Tannenbaumphänomen bei Skoliose & 0 \\
\hline 2. Tannenbaumphänomen bei Osteoporose & 0 \\
\hline 3. Wellenzeichen bei Osteoporose & 0 \\
\hline 4. Wellenzeichen bei Osteomyelitis & \\
\hline 5. Triangle Sign bei Osteomalazie & 0 \\
\hline & \\
\hline
\end{tabular}

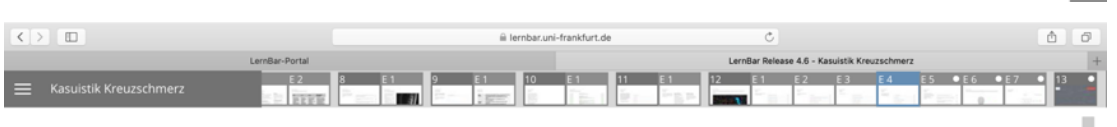

Kasuistik Kreuzschmerz

Frage 4

Welche der folgenden Tests und

Untersuchungen gehört NICHT $z$ Z
Untersuchung der Wirbelsăule?

\begin{tabular}{lc} 
1. Finger- Boden-Abstand & 0 \\
\hline 2. Lasègue-Zeichen & 0 \\
\hline 3. Inspektion der Kyphosen und Lordosen & 0 \\
\hline 4. Varus- und Valgus Stresstest & 0 \\
\hline 5. Palpation der Dornfortsätze & 0
\end{tabular}

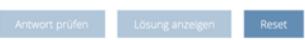

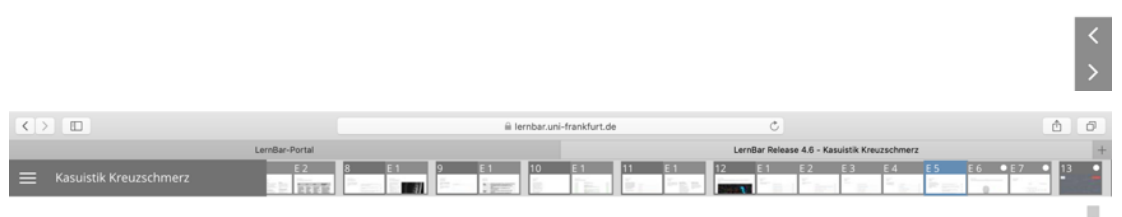

Kasuistik Kreuzschmerz

Frage 5

Eine 63-jährige Patientin leidet an

chronischen Rückenschmerzen, die durch

eine Osteoporose bedingt sind.Welche der

Kombinationen physikalisch-

therapeutischer Maßnahmen ist an

\begin{tabular}{|c|c|c|}
\hline & Eispackungen und tonisierende Klopfungen am Rücken & O \\
\hline 2. & $\begin{array}{l}\text { örtliche Wärmeanwendungen und krankengymnastische Krättigung der } \\
\text { Rückenmuskulatur }\end{array}$ & O \\
\hline 3. & $\begin{array}{l}\text { Unterwasserhochdruckstrahlmassagen und chirotherapeutische Manipulationen } \\
\text { der Wirbelsäule }\end{array}$ & b \\
\hline 4. & Suspension im Schlingenbett und Traktion der Wirbelsäule & \\
\hline & Bindegewebsmassagen und kalte Blitzgüsse & \\
\hline
\end{tabular}

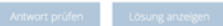




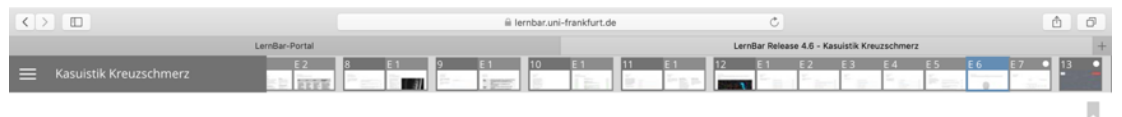

Super! Du hast die Kasuistik geschafft! Bitte evaluiere noch kurz die Kasuistik!
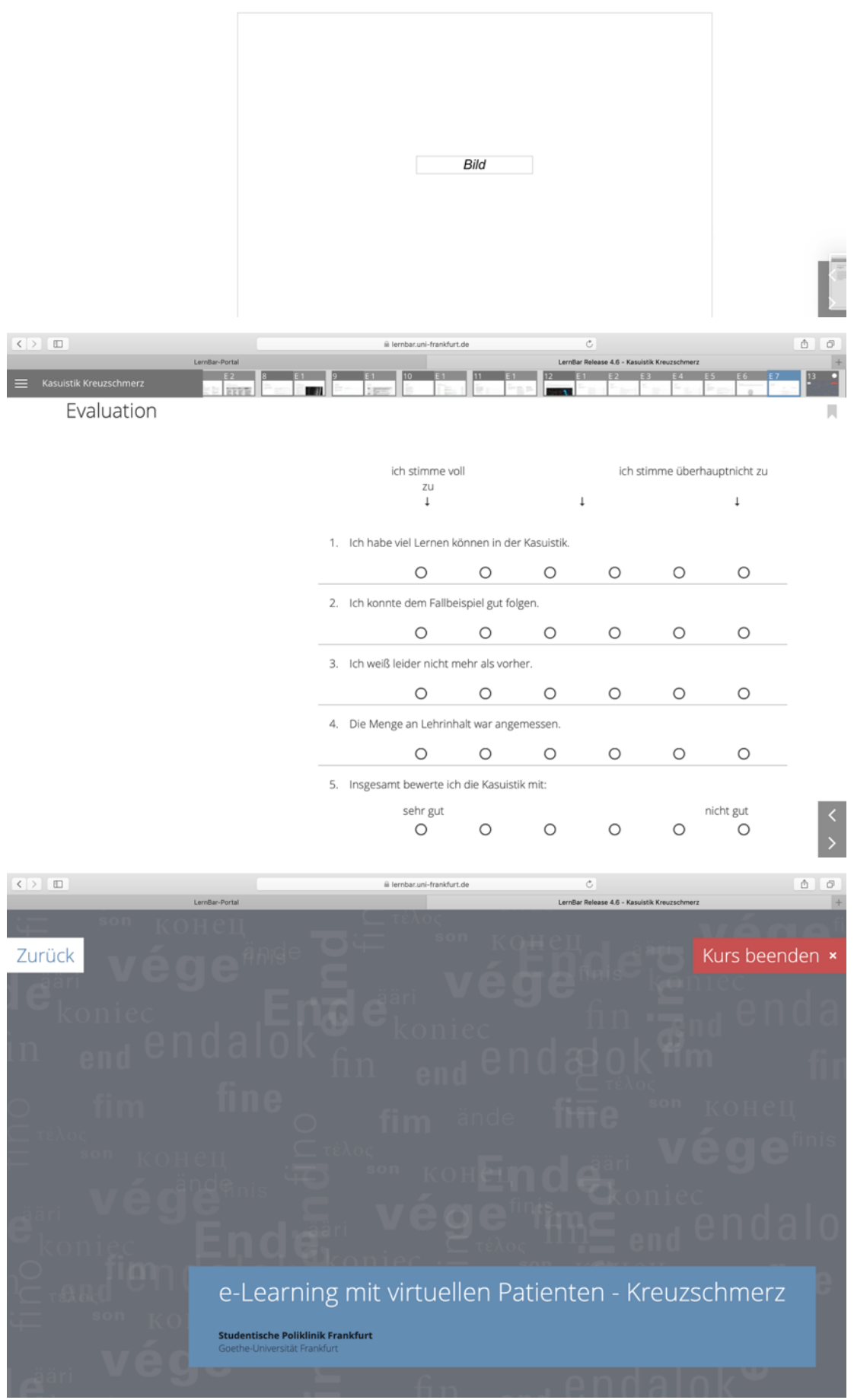
Kasuistik Polyurie

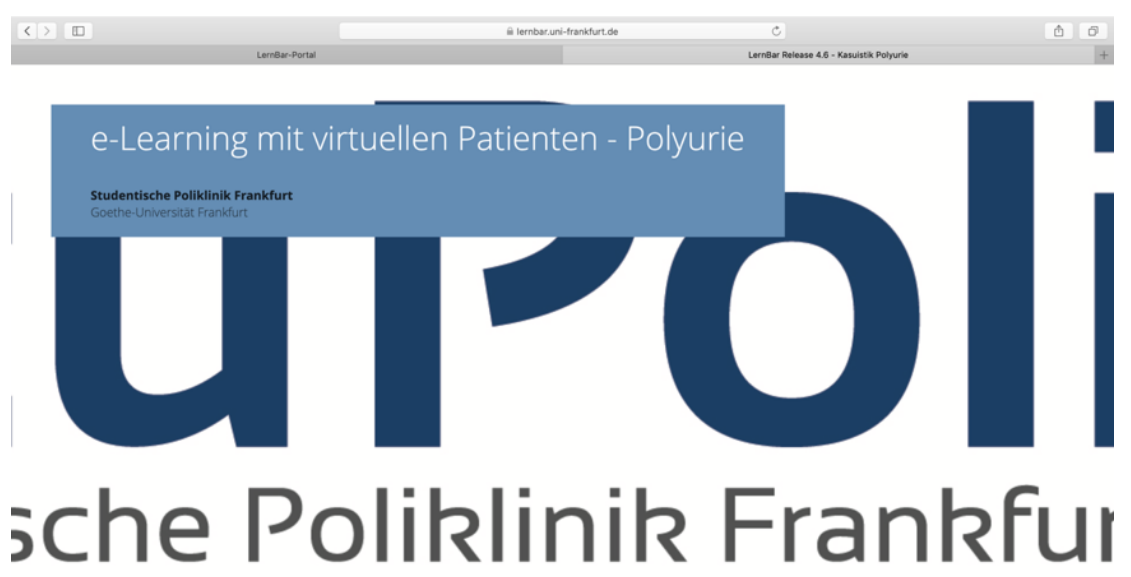

Goethe Universität KURS STARTEN

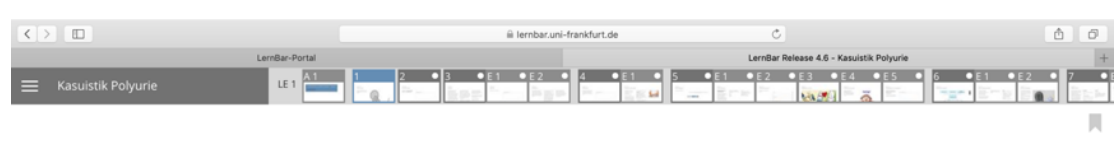

Kasuistik Polyurie

Thema: Polyurie

Willkommen zur Kasuistik Polyuriel Ohne

lange Einleitung starten wir direkt mit dem

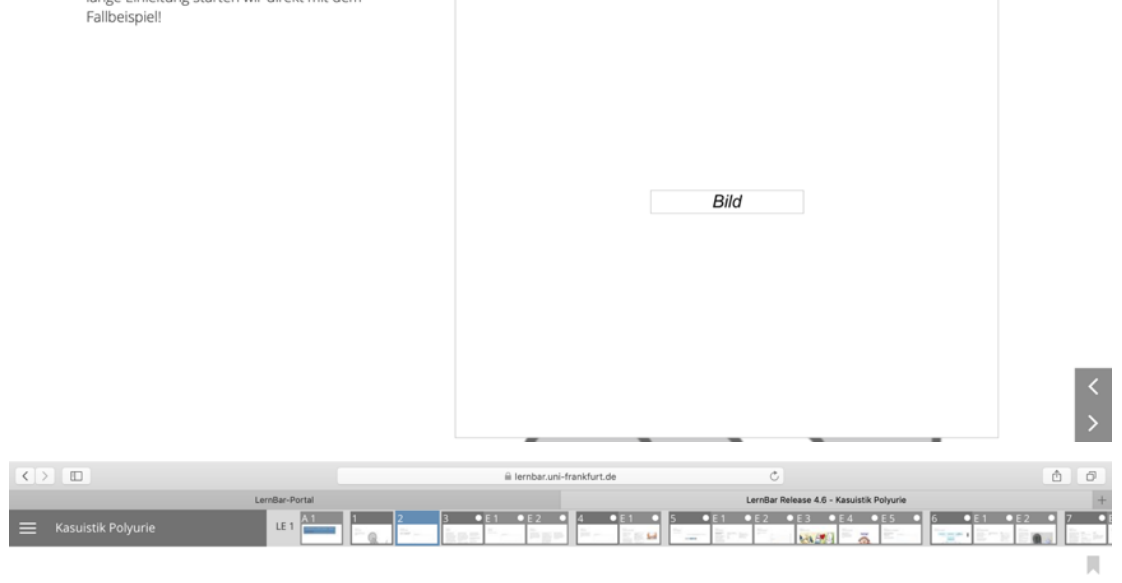

Kasuistik Polyurie

Persönliche Daten

Bitte schreibe in das Feld deinen Vor-und

Namen

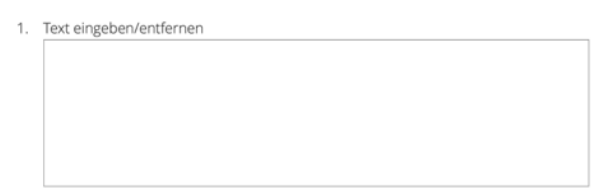




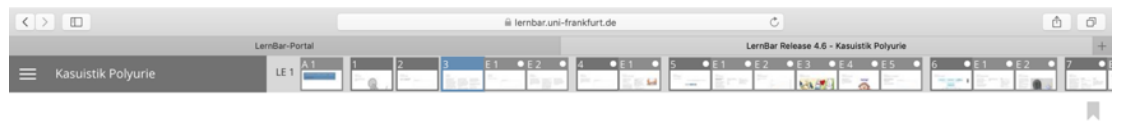

Kasuistik Polyurie

Fallbeispiel

Es ist Montag morgen und du hast in deiner Gemeinschaftspraxis für

Patienten im Wartezimmer. Als nächstes ist

ein Mann an der Reihe, der zum ersten Mal

in die Praxis kommt. Du erfăhrst, dass er

vor 20 Jahren aus Rumänien nach

Deutschland gekommen ist und selten zum

Arrt geht. Seine Kinder hatten inm jedoch

lassen. er müsste schließlich in lezter Zet

ötter auf Toilette.

Du beginnst mit deiner Anamnese!

Herr S. ist 58 Jahre alt und berichte Eigentich habe ich keine richtigen

gehen, das ist mir aufgefallen Ach ja und

ch hab immer furchtbar Durst... Ich glaube

das habe ich seit ungefährt einem halber Jahr. Ich arbette im Lager, in letzter Zeit bi Du fragst genauer nach und erfährst, dass
er Juckreiz (Pruritus) hat und dies auf seine "trockene Haut" zurückführt. Zu seinem Durstgefühl errahrst du, dass er täglich

etwa 4 Liter Wasser trinkt. Zusătzlich trinke er ab und zu Alkohol ('Abends ein Bier tu
Inm sind keinerlei Vorerkrankungen

Zeetrinnt, er hatte jedoch vor etwas längere

Finger amputiert wurden.

Herr S. ist verheiratet und hat 3 Kinder. Die Familienanamnese ergibt noch, dass sein

var - zucherk

Nikotinkonsum: 40 packyears,

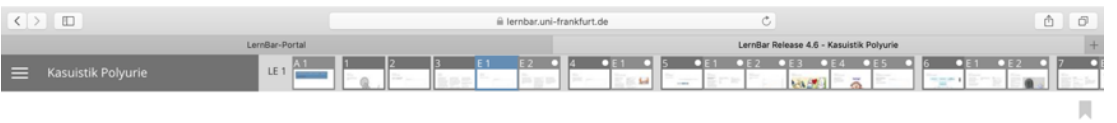

Kasuistik Polyurie

Anamnese

Was fehit uns noch in der Anamnese
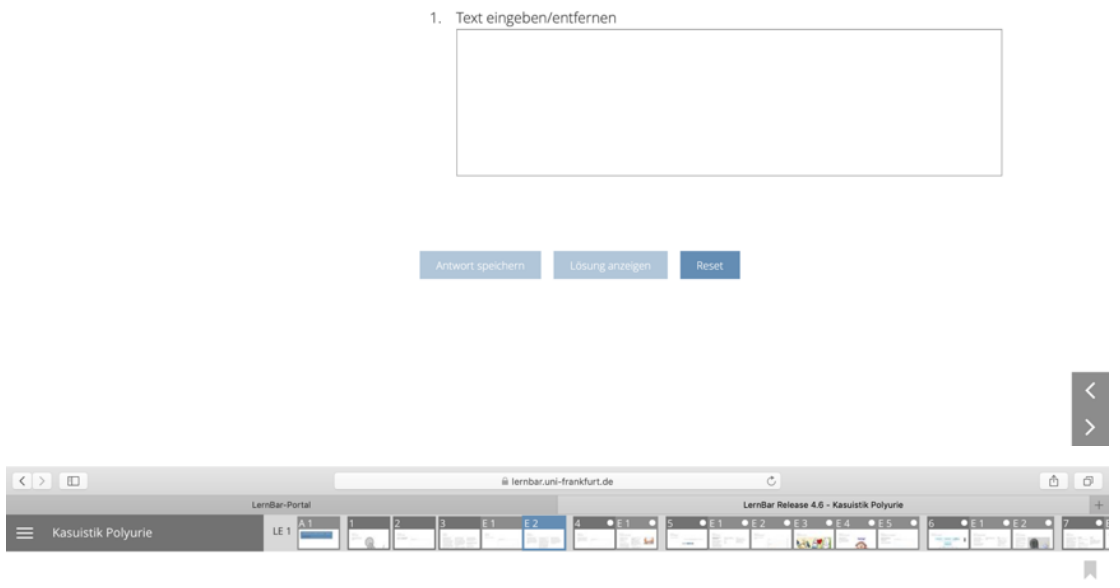

Kasuistik Polyurie

Risikofaktoren

Noch nicht erfragt wurden Genussmitte. genauere Angaben zur vegetativen Anaminese irgendwelche Sehstörungen vorhanden sind (warum, wirst du in der Kasuistik noch erfahren...)
Herr S. hat 40 packyears, andere Drogen abe er noch nie genommen.

oft Urin lassen zu

müssen, ist hier indiziert genauer

Wasserlassen? Wie sieht der Urin aus?

Es stellt sich heraus, dass er bis zu 8-10

mal am Tag auf Toilette geht, uber die

Menge hat er keine genauen Angaben

genaueres sagen Schmerzen oder

genaueres sagen. Sch
Brennen hat er nicht.
Zum Ernährungsverhalten gibt er folgendes

an: "Ich esse gerne Fleisch, meine Frau

Essen es schmeckt ja auch so gut und

Nachtisch gibt es auch immer. Sport mache ich nicht, die Arbeit ist ja anstrengend genug." 


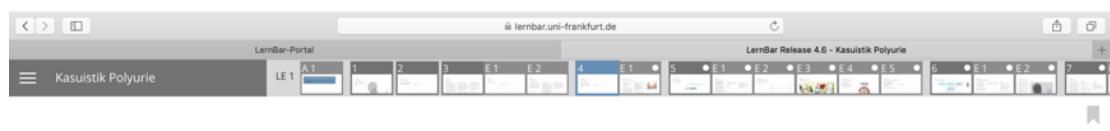

Kasuistik Polyurie

Weiter geht es

Die Anamnese ist geschafft und du hast

als nächstes erst mal die körperliche

Untersuchung durch. Was untersuchst do
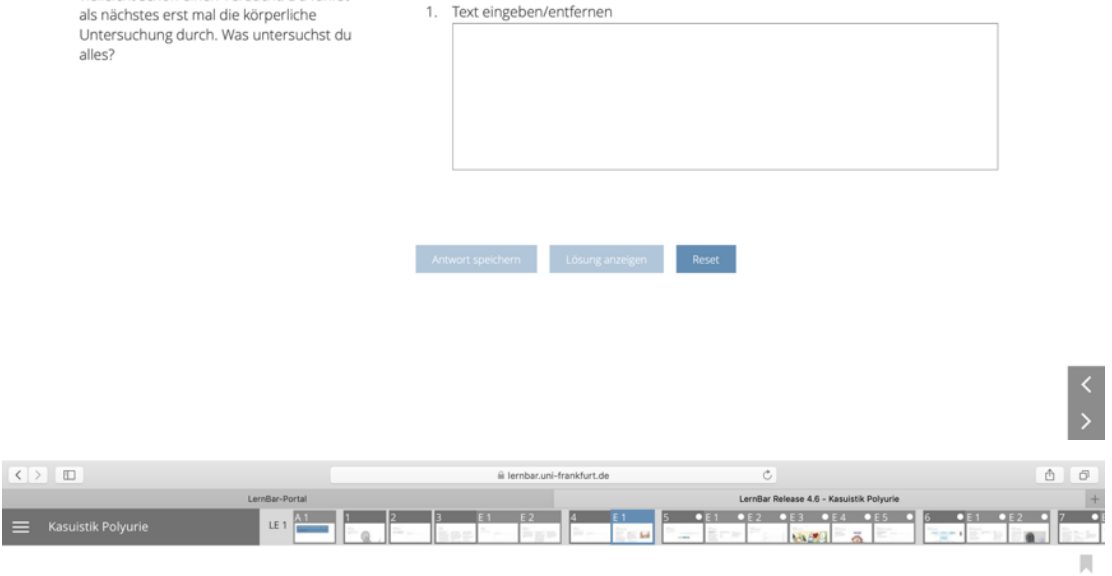

Kasuistik Polyurie

Körperliche Untersuchung

Du möchtest zunächst einmal die

Vitalparameter von Herrn $\mathrm{S}$. wis

- $\mathrm{BMI}=36.8 \mathrm{~kg} / \mathrm{m}^{\wedge 2}$

- RR 160/ 90 mmHG, Puls 94

Da er so einige Risikofaktoren mit sich

bringt, (Raucher, Adipositas, Hypertonus

untersuchst du auch Herz, Lunge und

mal dien. Au def rogenden Sele noch en

sicher schon bekannt sind Du kannst sie.

die optional zur Wiederholung anschauen.

Dir fallt bei der Untersuchung eine seh

trockene Haut au

cht Darmgetāuscht über

gablähter Bauch

Pulmo: sonorer Klopfschall, beidseitis

belüttet, vesikuläres Atemgeräusch

Cor: normofreuquent, rein und rhythmish

Heratome, heme vilien

Hier findest du noch mal Wiederholungen

zur Durchführung einer kôrperlichen

Unterusuchung:

Herzuntersuchung ( $(\mathrm{wmw})$

cungenuntersuchung (

Bild

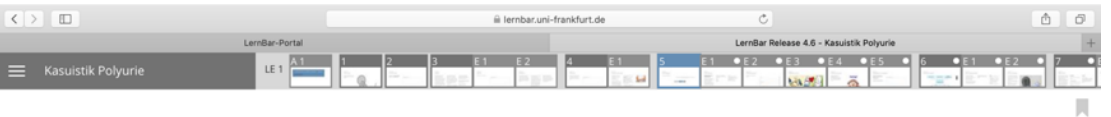

Kasuistik Polyurie

Verdachtsdiagnose

Was ist deine Verdachtsdiagnose?

1. Text eingeben/entfernen Text eingeben/entfernen 


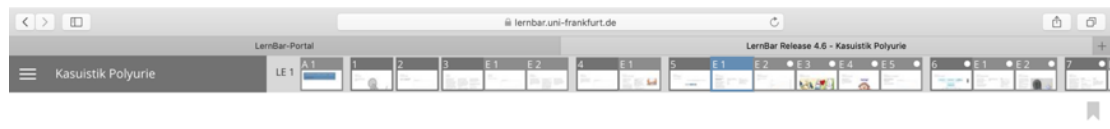

Kasuistik Polyurie

Verdachtsdiagnose

Verdacht: Diabetes mellitus - der "honigsüße Durchfluss"

Ganz genau, mit deiner Anamnese konntest du bestimmte Symptome, die für einen Diabetes sprechen könnten herausfinden. Die körperliche

Untersuchung hingegen hat außer den

Zeichen der Exsiknose keine direkten

Hinweise ergeben, vielmehr sprechen die
Bei folgenden Verdachtssymptomen solltest du nach einem Diabetes fahnden: Gewichtsverlust

Durst

Polyurie Múdigkeit
Pruritus
Wichtig:

Ein Typ- 2- Diabetes ist selbst im

Manifestationsstadium oft stumm. Der

metabolische Vor- und

Begleiterkrankungen geprägt! 


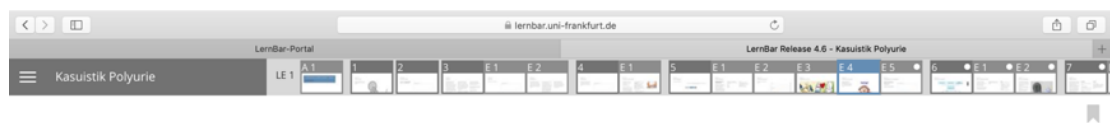

Kasuistik Polyurie

Problem Überernährung

Überernährung mit Adipositas und

Bewegungsmangel sind die

des Typ-2-Diabetes mellitusi Ca. 8006 der

Typ-2-Diabetiker sind übergewichtig!

(Herold)

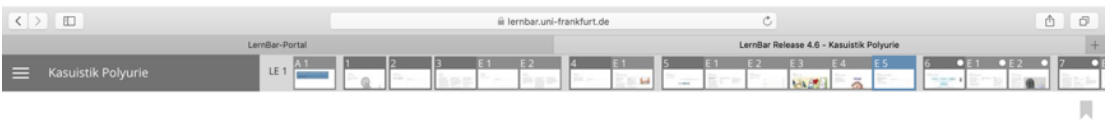

Kasuistik Polyurie

Bestätigung der Verdachtsdiagnose

Wir haben eine "passende Klinik" zum

Diabetes, jetze git es diesen aberwetter

Wie funktioniert das?..

Wie gehst du mit der weiteren Diagnostik

1. Text eingeben/entferner

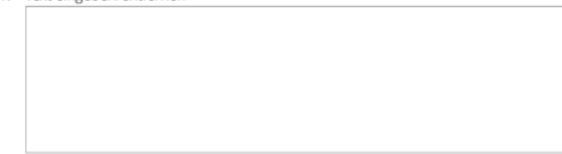

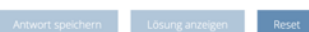

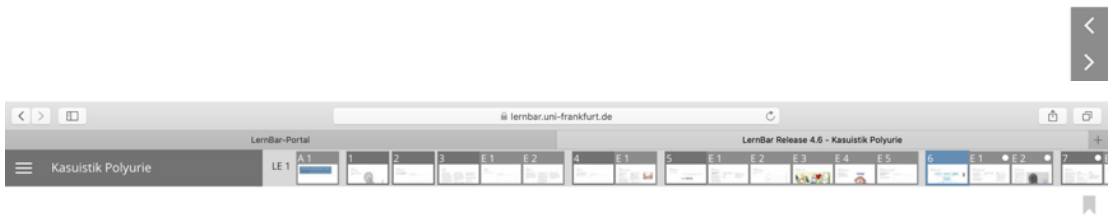

Kasuistik Polyurie

Diagnostisches Vorgehen 


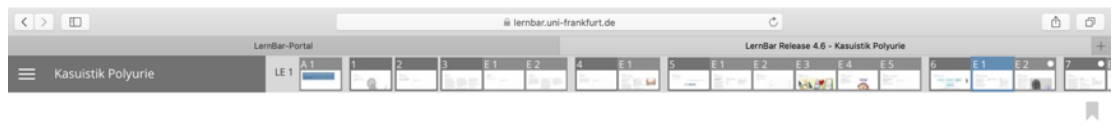

Kasuistik Polyurie

Weitere diagnostische Mittel

Urin: Mikroalbuminurie ist DAS Frühzeichen einer diabetischen Nephropathie. Auch

nicht zur Diagnosesicherung geeignet.

C. Peptid-Bestimmung dient z.B. der C-Peptid-Bestimmung dient z.B. der Diabetes.

in wird in B-Zellen des Pankreas

(nsulin an Ribosomen

Modifikation zu Proinsulin am ER

$\rightarrow$ Abspaltung C-Peptid $\rightarrow$ relfes Insulin

- Typ 1 DM: durch absoluten

Spezinsche Autoantikorper kann man be

Insulinmangel ist C. Peptid kaum

$\rightarrow$ Typ 2 DM: C. Peptid ist eher erhöh.

Typ-1- Diabetes mellitus bestimmen,
jedoch keine Routine- Untersuchung

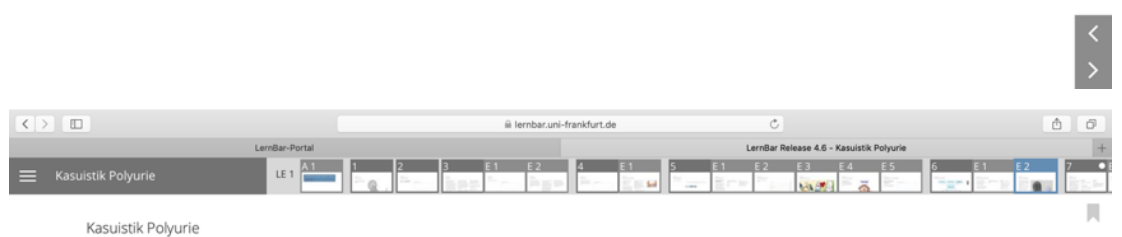

Zurück zum Fallbeispiel

Wir bestimmen den Blutzuckerspiegel vo Herrn S., welcher bei $174 \mathrm{mg} / \mathrm{dll}$ liegt, auf Nachifage meint er, dass ervor 2 stunden nicht nüchtern. Außerdem nehmen wir Blut ab, um den HbA1C- Wert und auch die Triglyceride zu bestimmen.

Wir bestellen Herrn Simi für Ende dieser Woche noch einmal ein, um seine

Bis dahin überlegst du dir noch mal wie genau das noch mit den Diabetes. Typen Bild war.
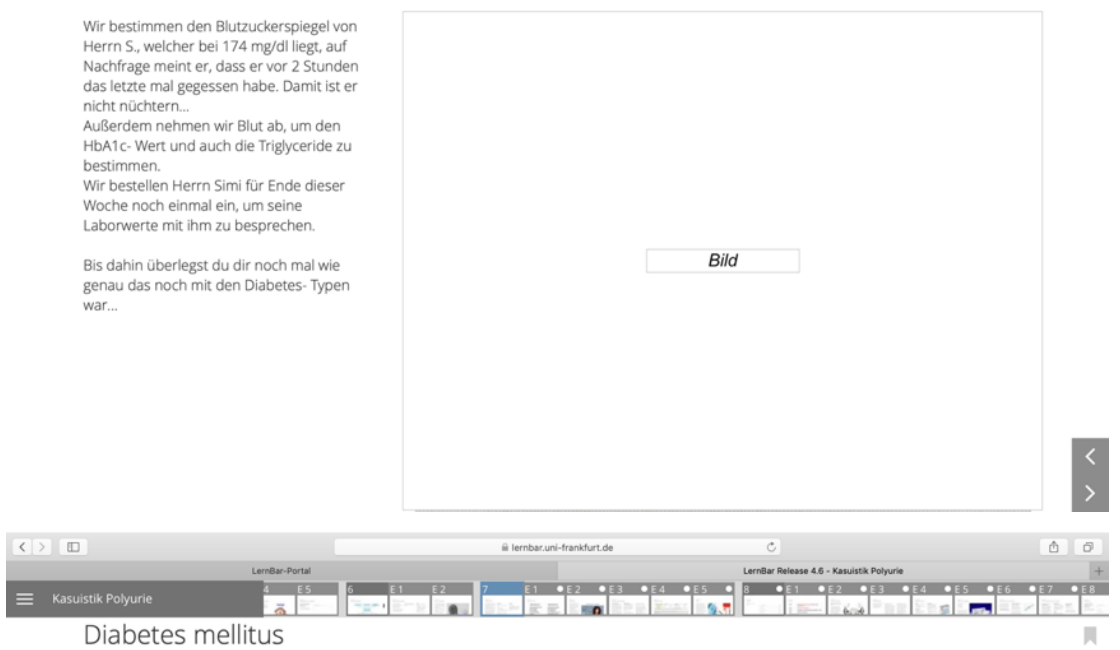

\section{Klassifikation:}

Nach WHO und ADA (American Diabetes

1. Diabetes mellitus Typ 1 3. weitere spezifische Diabetes- Typen (Typ 3)
- z.B.

$-2 . B$. MODY $=$ Maturity - onset diabetes of the young

Erkrankungen des exokrinen Pankreas, Endokrinopathien

4. Gestationsdiabetes (Typ 4)

Wir schauen uns in dieser Kasuistik den Typ

1 und den Typ 2 Diabetes

\section{Symptome:}

Allgemeine Symptome

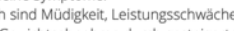

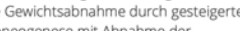
Muskelmasse sowie Kalorienverluste über den Urin.

sowohl eine starke genetische

Wohlstandsgesesellschaften. Eine gestörte

Wirkung des insulins an den Korpp

(insulinresistenz) und eine (zunachst

verminderte Insulinsekretion der $\beta$ Z Zellen

führen hier zur Hyperglykämie. Dieser

Diabetestry bleibt fatalenweise häufig ïber

viele Jahre klinisch inapparent, führt aber

bereits durch

Stoffwechsellage uber Mikro- und

Makroangiopathien zu schwerwiegenden

Herz. Kreislauf, Nieren, Augen und 


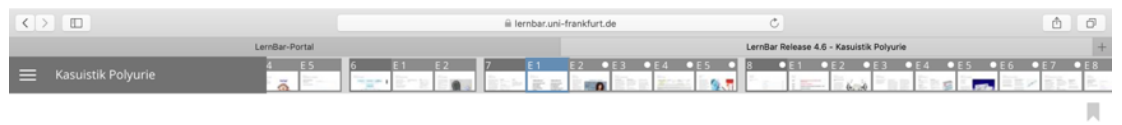

Kasuistik Polyurie

Typ 1 versus Typ 2

\section{Diabetes mellitus Typ 1}

- rasche Manifestation bei oft jungen Patienten

- Coma diabetikum

- oft nach Virusinfektion auftretend

\section{Diabetes mellitus Typ 2}

- schleichende Entwicklung bei meist älteren pat.

- oft "Zufallsbefund"

- erst im Verlauf kommt es zur hyperglykämie, anfangs auch Hypoglykämie möglich

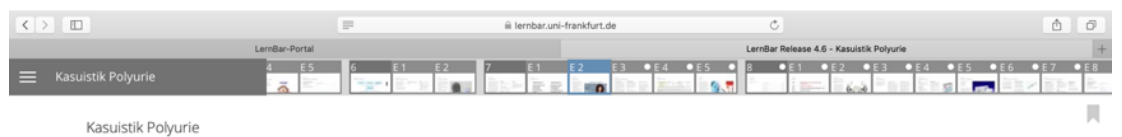

Epidemiologie

Zurzeit ist eine steigende Prävalenz zu beobachten, insb. fur den Typ-2-Dib

prognostiziert. Ursächlich sind die wet

verbreitete Gevichtszunahme und der

Bewegungsmangel in den
Wohlstandsgesellschaften

In der Altersgruppe bis zum 50. Lebensjah liegt die Próvalenz bei ca. $5 \%$ der

Gesamboevolkerung, in der Atersgruppe

der Altersgruppe der 70-79-Järrigen

schließlich bei ca. $23 \%$

Key Messages:

-1 von 11 Erwachsenen hat Diabetes (415

- alle 6 Sekunden stirbt ein Mensch an

Diabetes (-Folgeerkrankungen

Diabetes haben $(642$ Millionen)

(Quelle: www.diabetesatlas.org ( $(\mathrm{mw}$ ))

Bild

http: //nwww.diabetesatlas.org (nww) - zum Nachschlagen

Bild

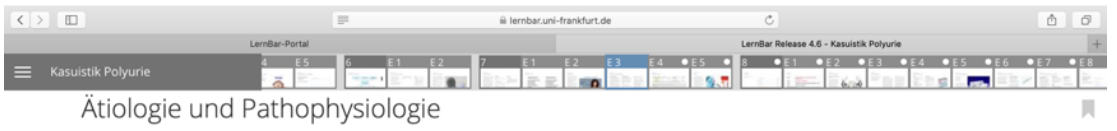

Typ 1- Diabetes mellitus:

Dieser ist meist autoimmun vermittelt, es

liegt eine progrediente Zerstorung der

insulinproduzierenden pzelentinden

Dies führt zum absoluter Insulinmanget.

Erst bei Zerstörung von $80 \%$ der $\beta$-Zellen

steigt dann der Blutzucker an.

Es ist außerdem eine HLA-Assozziation

vorhanden. Ca. $10 \%$ der Erkrankten haben

eine postive familienanamnese, menr als

Assozizen a chatheristischent

4.6-fach erhöhtes Risiko gegenüber Nicht-

Genträgern)
Diabetes mellitus Typ 2: ist meist mit metabolischem Syndrom assoziliert und besizt eine starke erblich . Wahrscheinlichkeit im Verlauf seines Lebens einen Typ-2-Diabetes zu entwicken. Kardinale Risikofaktoren für die Manifestation eines Diabetes mellitus Typ

-Stammbetonte Adipositas (Messung des Bauchumfangs): Die Menge des viszeralen ettes ist insb. beim männliche

Geschlecht ein Risikofaktor
Fettstoffwechselstôrungen (Trigyzeride HDL-Cholesterin $\downarrow$ )

Nachweis einer gestörten Glukosetolera
Mehrere Faktoren spielen bei der

Entwicklung eines Typ-2-Diabetes eine

Rolle

Zugrunde liegt zunächst eine periphere

Prädisposition zusätrzlich verstärkt durch

Adipositas. Zu Beginn der Erkrankung kann

die Insulinresistenz durch eine

kompensatorisch gesteigerte

insulinsekretion ausgegitchen werden (evt.

Autreten von postprandialen

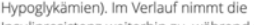

die insulinsekretionskapazităt verringert.

Nach einer Phase gestörter

Glukosetoleranz kommt es zu

postprandialen Hyperglykämien, im

weiteren Verfauf manifiestiert sich ein

Diabetes melitus Typ 2 mit pathologisc

Insulinresistenz ist die Aufnahme von

Glukose in die Muskel- und Fettzellen

vermindert, was eine Hyperglykämie

begünstigt.

Zusätzlich kommt es zur Förderung der Hypergylyämie bedingt durch den Weg 


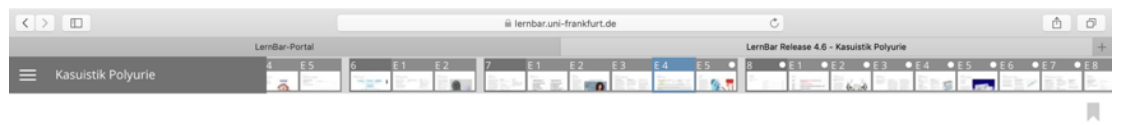

Kasuistik Polyurie

Diagnosesicherung

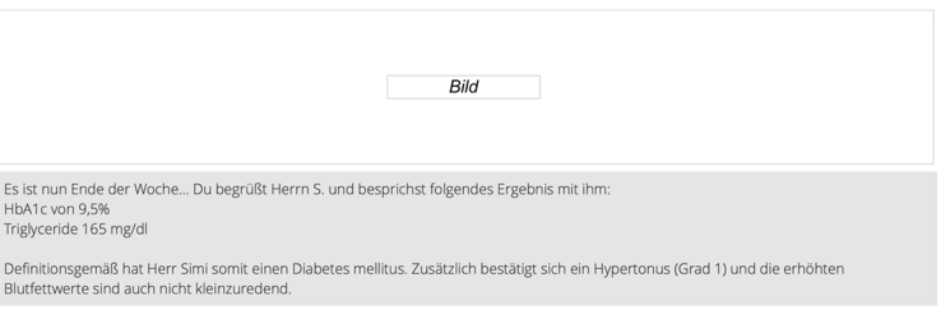

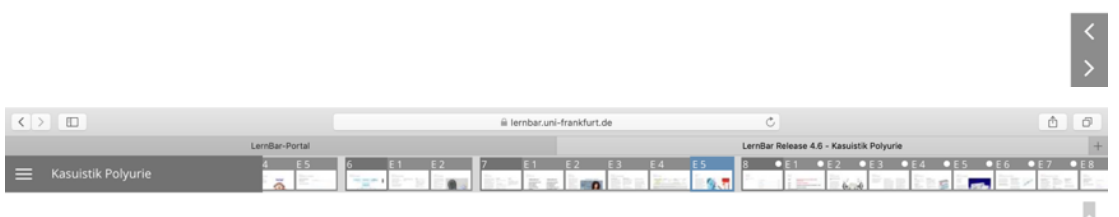

Kasuistik Polyurie

$\mathrm{HbA} 1 \mathrm{c}$

Das HbAic entstent durch nicht-

enzymatische Bindungvon Glukose

irreversibel ist, wird sie erst durche

Abbau der Exythrozyten eliminiert. Daher

korreliert die Höhe des HbA1c mit dem

mittleren Blutglukosegehalt der

vergangenen 8-12 Wochen. Früher wurde

die Bestimmung von HbAic insbesondere

zurventautskontrolie verwendet,

Primärdiagnostik empfohlen Vorteile sind

das fehlende Nüchternheitsgebot und eine

tageszeitunabhāngige Bestimmbarkeit.

Also wir merken uns: der HbA1C dient uns

zur Beurteilung der Blutglukoseeinstellung
der letzten 2.3 Monate!!!

Bild

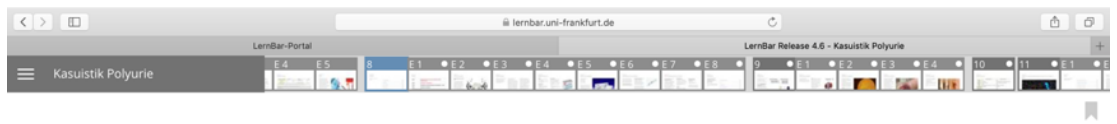

Kasuistik Polyurie

Therapie

Was können wir nun mit Herrn s.

\begin{tabular}{lc} 
1. Insulintherapie einleiten & $\square$ \\
\hline 2. "Lifestyle ändern" & $\square$ \\
\hline 3. Hypertonie behandeln & $\square$ \\
\hline 4. Tabakentwöhnung & $\square$ \\
\hline 5. Kombinierte antidiabetische Therapie & $\square$
\end{tabular}

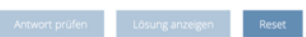




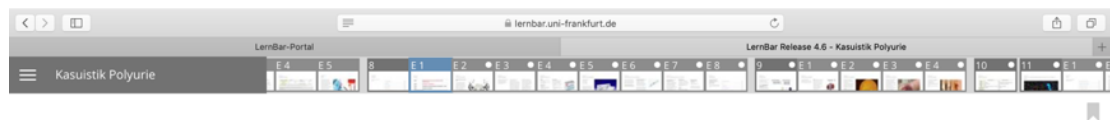

Kasuistik Polyurie

Therapie

\begin{tabular}{|c|c|}
\hline Stufe 1 & $\begin{array}{l}\text { Basistherapie (Ernährung, Schulung, körperliche Aktivität, } \\
\text { Tabakentwöhnung) }\end{array}$ \\
\hline Stufe 2 & Monotherapie 1. Wahl Metformin \\
\hline Stufe 3 & Antidiabetische Kombinationstherapie oder Antidiabetikum + Insulin \\
\hline Stufe 4 & Intensivierte Insulintherapie \\
\hline \multicolumn{2}{|c|}{$\begin{array}{l}\text { Stufentherapie bei Typ- 2- Diabetes } \\
\text { Wir müssen be Heirn S. mehrere Baustellen anpacken: } \\
\text { - "Lifestyle" (Körperliche Aktivität, Nikotinverzicht, u.a.) } \\
\text { - erhöhte Triglyceride } \\
\text { - Bluthochdruck } \\
\text { - Diabetes }\end{array}$} \\
\hline \multicolumn{2}{|c|}{$\begin{array}{l}\text { Im Bild siehst du die Stufentherapie bei Typ- 2- Diabetikern. Wir beginnen mit Stufe 1, welche die Grundlage der Therapie bildet, außerden } \\
\text { bekommt Her S. von uns auch Metformin, ein Antidiabetikum. } \\
\text { Wir verschreiben inm: Metformin } 500 \mathrm{mg} \text {, Simvastatin } 20 \mathrm{mg} \text { und Ramipril } 2,5 \mathrm{mg} \text {. Außerdem wird ein Termin zur Wiedervorstellung und } \\
\text { Verlaufskontrolle vereinbart. } \\
\text { Im Vordergrund steht es die kardiovaskulären Risikikfaktoren zu mindern und den HbA1c auf } 6,5-7,5 \% \text { zu senken! }\end{array}$} \\
\hline
\end{tabular}

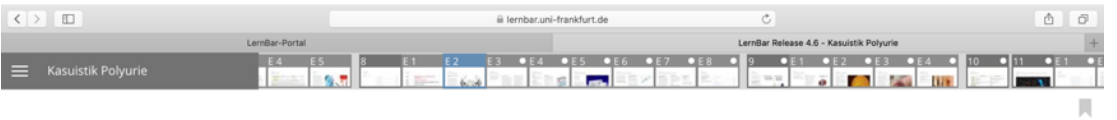

Kasuistik Polyurie

Vielschichtige Therapie

Unterschiede zwischen Typ 1 und Typ 2

Die Therapie des Diabetes mellitus ist komplex, vielschichtig und nicht zulets auch abhângig von der vorliegenden Diabetesform. Grundsätzlich ist beim Typ. 1-Diabetiker immer eine Insulintherapie erforderlich. Fur Typ-2-Diabetiker gibt es (Gewichtsreduktion, Antidiabetikg do beim Typ-1-Diabetiker allesamt unwirks sind.

In den folgenden Abschnitten lernst du

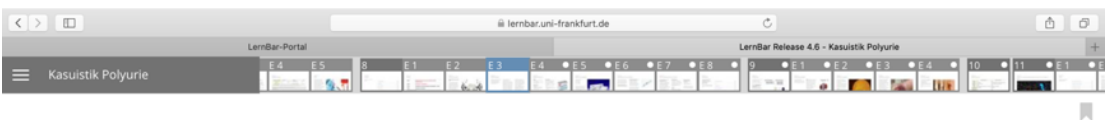

Kasuistik Polyurie

Orale Antidiabetika

Antidiabetika sind alle pharmakologischen Wirkstoffe außer insulin, die zur

Ratzeneren

Führen Lebensstiländerungen

Gewichtsreduktion durch
Ernährungsumstellung und körperliche

(1)

einer ausreichenden Senkung des $\mathrm{HbA} A \mathrm{C}$

Wertes (Ziel-HbA1c 6,5-7,5\%), wird die

medikamentöse Therapie begonnen. Der

wichtigste Wirkstoff und das Mittel der 1 .

Wahi bel allen Typ-2-Diabetikern ist das

Biguania Mettrom min. Es west gunstige

führt zur gewünschten Nebenwirkung

Gewichtsreduktion und zeigte in

Endpunktstudien den größten Nutzen bzg.

Mortalităt und Právention von

Langzeitkomplikationen des Diabetes

Eine gefürchtete Nebenwirkung von

Metormin ist die Lakataziose, die insb

schwerer Erkrankungen und Opermentionen

sowie bei intravenöser Gabe jodhaltiger

Kontrastmittel auftritt. Daher muss

Metformin bei Vorliegen dieser

Risikofaktoren abgesetzt und i.d.R. durch

eine Insulintherapie ersetzt werden.

Therapie mis Metformin hin erhötes

Risiko für lebensbedrohliche

Hypoglykämien besteht. 


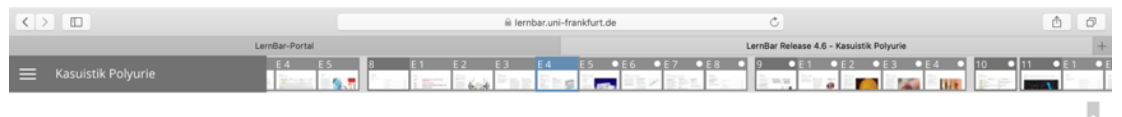

Kasuistik Polyurie

Biguanide (Metformin)

Wirkung:

Wetormin verstärkt die Insulinwirkung

durch Erhöhung des

nsatzes (keine Erhöhun

der Insulinsekretion!)

Nebenwirkungen:

Laktatazidose

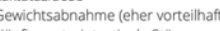

.

\section{Kontraindikation:}

alle Situationen mit Hypoxämie oder

zidotischer Stoffwechseltege da sor

Gefahr der Laktatazidose besteht

$\rightarrow$ Nierenversagen bzw. diesbezúgliche

Risikosituationen

$\rightarrow$ Chronische Niereninsuffizienz (GFR

$<30 \mathrm{~m} / \mathrm{min})$

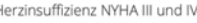

Chronische respiratorische Insuffizien
Bild

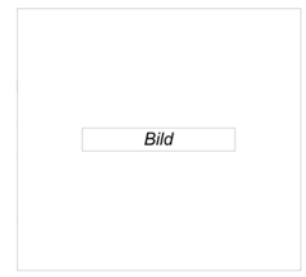

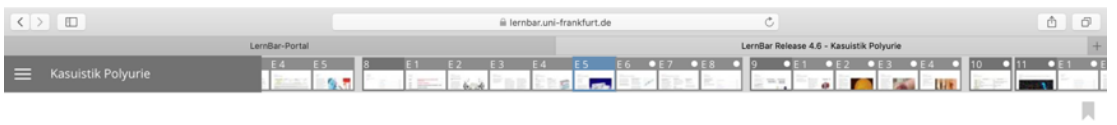

Kasuistik Polyurie

Weitere orale Antidiabetika

- Sulfonylharnstoffe (Cave

Hypoglykämierisike

GLP-1-Analoga

-SGLT-2-Inhibitoren

Der Übersichtlichkeit des Themas, reicht es

wenn du Metformin kennst, ) Diese

Medikamente sind nur der Volistandigkeit

hier (nww) mehr nachlesen.

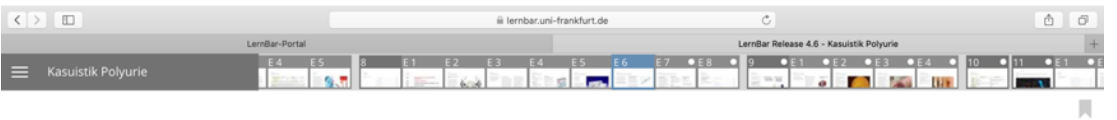

Kasuistik Polyurie

Insulintherapie beim Typ-1-Diabetes

leder Typ-1-Diabetiker bekommt eine

insulintherapiet insulintherapien sind

oeschut werden a ardem wird immer

individuell eine Therapie angepasst

Intensivierte Insulintherapie: Versuch

Ginen nahezu physiologischen

$\rightarrow$ Intensiverte konventionelle

insulintherapie (Basis- Bolus- Prinzip)

Basal unterstutzte orale Therapie (BOT)

Konventionelle Insulintherapie: feste

- Supplementäre Insulintherapie

Bild

Diese sind hier der Vollständigkeit halber

genannt, es schadet nicht davon mal

sehoriell und zun, ist jedoch auch etwas

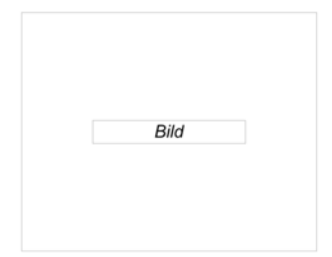




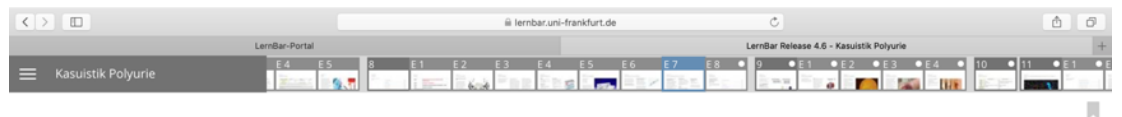

Kasuistik Polyurie

Exkurs: Komplikationen bei Typ-1-Diabetes

Als wohl wichtigste Komplikation sei das

Ketoazidotische Koma gen

Hypergylyămie sowie einer gesteigerten

Lipolyse. Beide Mechanismen gehen mit

einem progressiven Volumenverlust einher.

Die Lipolyse mit Bildung von Ketonkörpern

aus den figesetzten fettsauren funnt

auserdem zur Ausbildung

Oft als Erstmanifestation eines Diabetes

mellitus! Notfallsituation, die einer

intensivmedizizischen Therapie unterliegs
Pathomechanismen:

Insulinmangel $\rightarrow$ Hyperglykämie $\rightarrow$ Hyperosmolaritát $\rightarrow$ Osmotische Diurese

Ketonkörperbildung $\rightarrow$ Ketose $\rightarrow$

Symptome:
Polyurie, Polydipsie, Übelkeit, Erbreche Exsikkose, Hypotonie, Kollapsneigung Bewusstseinstrübung esondere Symptome des

Zügiger Beginn
Bauchschmerzen

Nach Azeton riechender Atem

- Kusmaul-Atmung

Achtung: Es gibt auch ein Diabetisches dann von einem hyperosmol

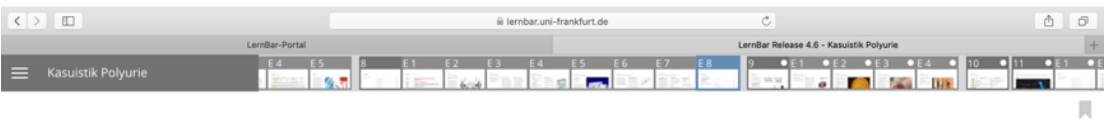

Kasuistik Polyurie

Komplikationen

Neben den akuten Komplikationen

yperglykamien bis hin zum

Vietzahl an langfristigen got es ein

Folgeerkrankungen.

Was ist deiner Meinung nach die häufigste

Komplikation eines Diabetes mellitus (Typ1

\begin{tabular}{lc} 
1. Diabetische Retinopathie & 0 \\
\hline 2. KHK & 0 \\
\hline 3. Aortendissektion & 0 \\
\hline 4. Diabetische Nephropathie & 0 \\
\hline 5. Diabetische Lunge & 0
\end{tabular}

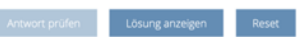

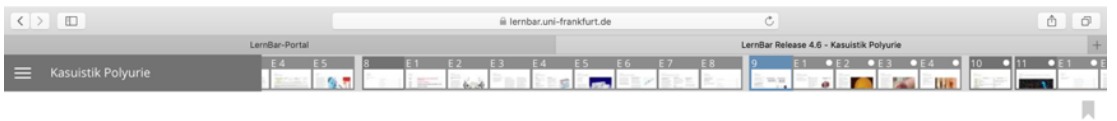

Kasuistik Polyurie

Komplikationen

Ca. 1/3 der Patienten mit Diabetes mellitus

(Typ 1 und 2III) entwickelt im

Nephropathie Dazu im folgenden metr.

Man unterscheidet ganz grob zwischer

diabetischer Makroangiopathie und

Mikroangiopathie. in der unteren Taben

slehst du was alles zu den Angiopathien -

anderer Komplikationen.

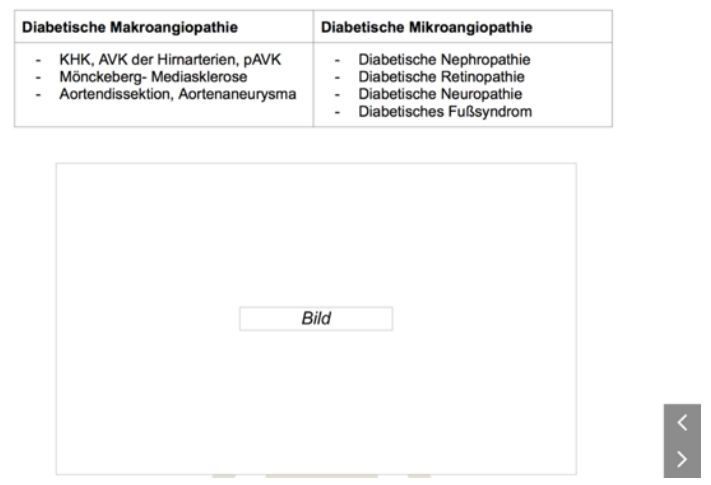




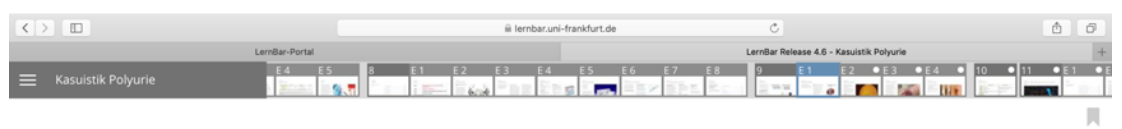

Kasuistik Polyurie

Diabetische Nephropathie

Frühsymptom der diabetischen

Nephropathie ist die Mikroalouminurie $(G)$.

der Höhe des kardiovaskulüren Risikost

Klinik: Die Glomeruläre Filtration ist initid

erhöht (Hyperperfusion), anschließend

kommt es zunehmend zu, Abfall. Eine

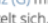

Oft existiert zusätzlich eine arterielle

Hypertonie, die fruhzeitige antihypertensive

Therapie verzogert die progression der

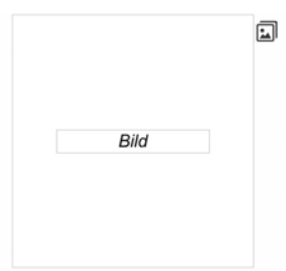

司

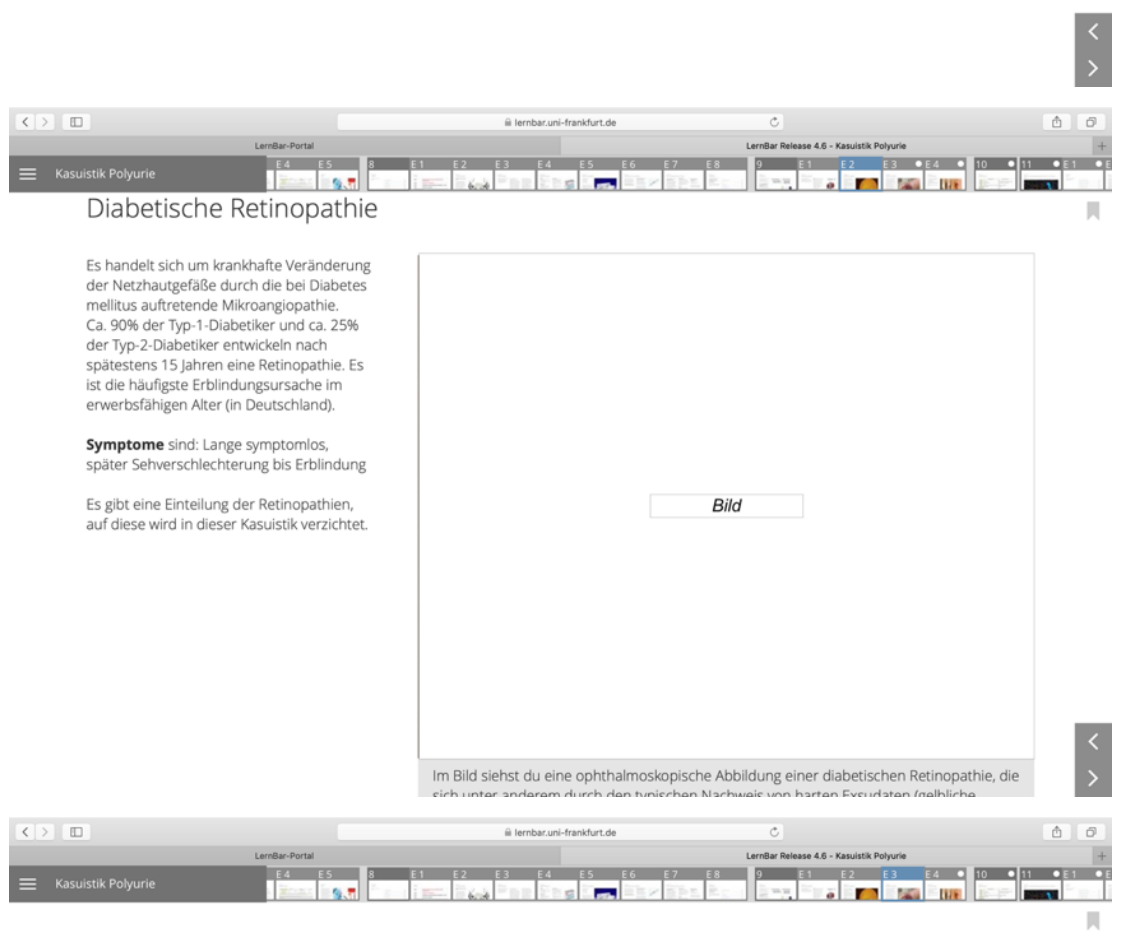

Kasuistik Polyurie

Diabetisches Fußsyndrom

Der neuropathischer dialektischer Fuß ist die häufigste Form des diabetischen Fußes

Vibrationsempfinden vermindert und auch das Schmerz- und Temperaturempfinden sind ger

Als Komplikation kann siche ein Malum

perforans $(G)$ entwickeln. Dies ist ein Ukus infolge einer Nervenschädigung.

Polyneuropathie. Es imponiert als

schmerzloses Geschwưr, hăufig

Fußballen oder Ferse, und kann

Ausgangspunkt fưr eine lebensgefährliche

Bild

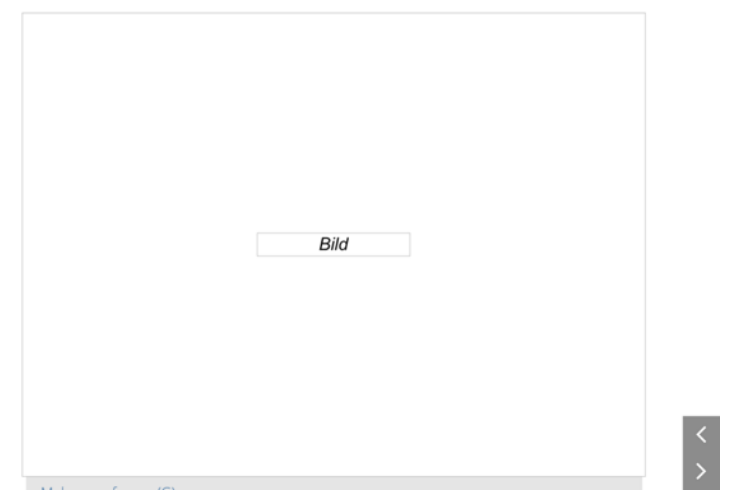




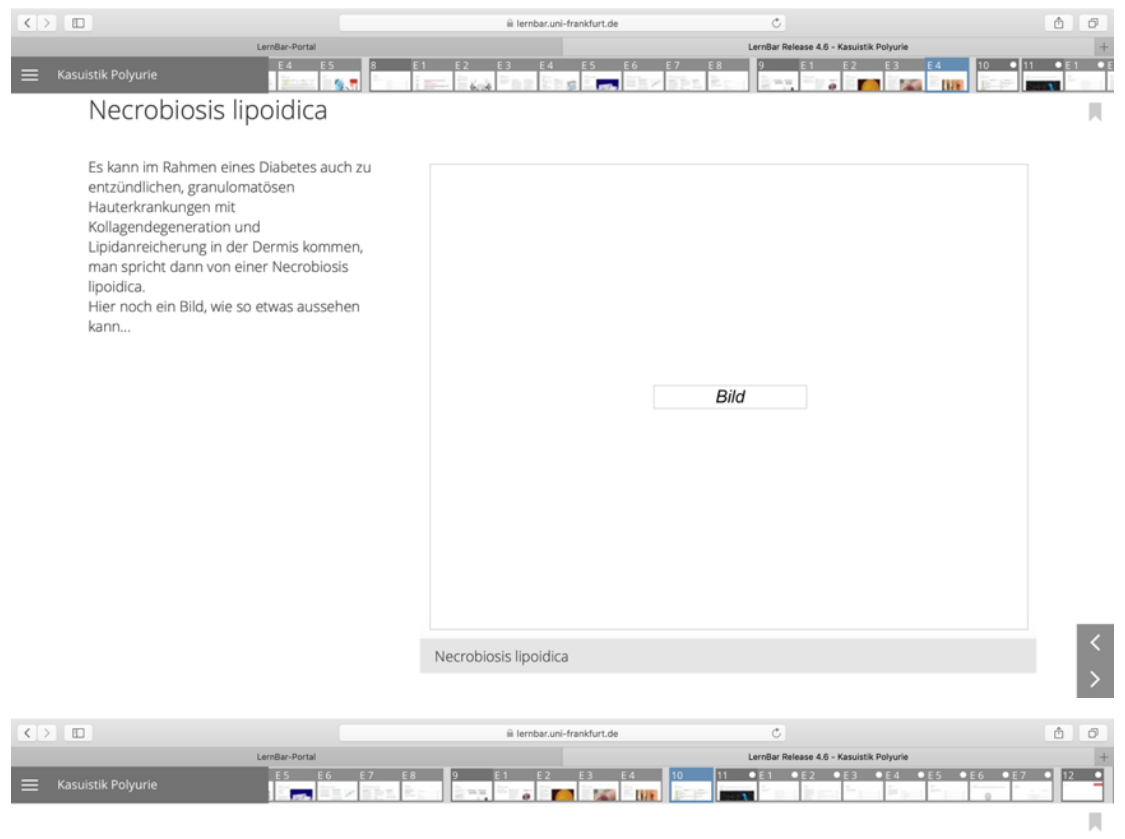

Kasuistik Polyurie

Zusammenfassung
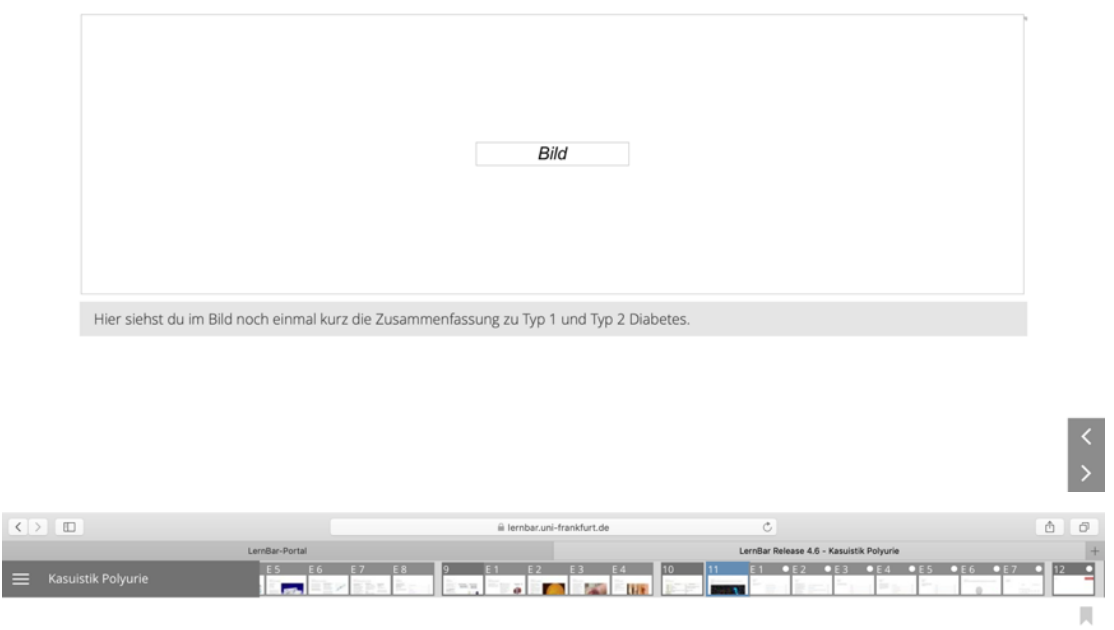

Kasuistik Polyurie

Super, nun ist die Kasuistik abgeschlossen. Zum Schluss noch 5 Fragen zum üben :) 


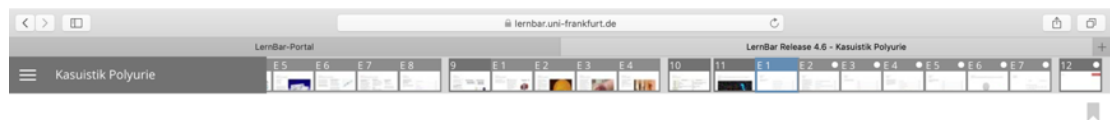

Kasuistik Polyurie

Frage 1

Was gehört nicht zum sogenannten

Metabolischen Syndrom?

\begin{tabular}{lc} 
1. Hypertonie (systolisch $>130 \mathrm{mmHg})$ & 0 \\
\hline 2. niedriges LDL-Cholesterin $(<50 \mathrm{mg} / \mathrm{dl})$ & 0 \\
\hline 3. erhöhte Triglyceride $(>150 \mathrm{mg} / \mathrm{dl})$ & 0 \\
\hline 4. Nüchternblutzucker $>100 \mathrm{mg} / \mathrm{dl}$ & 0 \\
\hline 5. Typ- 2 Diabetes mellitus & 0
\end{tabular}

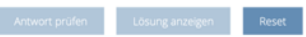

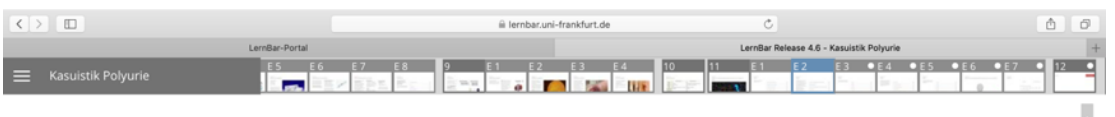

Kasuistik Polyurie

Frage 2

Ein 56-jähriger Patient wird seit 30 Jahren

wegen Typ-1-Diabetes mit insulin

Er murde im terten Mosne a) behandek.

versorgt. Seine Ehefrau gibt an, dass

anders als früher, die Hypoglykämien nicht

bemerkt habe und keine

Gegenmaßnahmen habe treffen

Konnen.was konnte ame ehesten der Grund

der Hypogykämien sein?

\begin{tabular}{lc} 
1. Neuropathie mit Beteliligung des autonomen Nervensystems & O \\
\hline 2. Nebennierenüberfunktion & 0 \\
\hline 3. besonders schnelle Absenkung des Blutzuckers von einem hohen Niveau & 0 \\
\hline 4. Hyperthyreodismus & 0 \\
\hline 5. Wachstumshormon- Überproduktion (Akromegalie) & 0
\end{tabular}

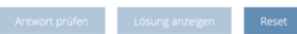

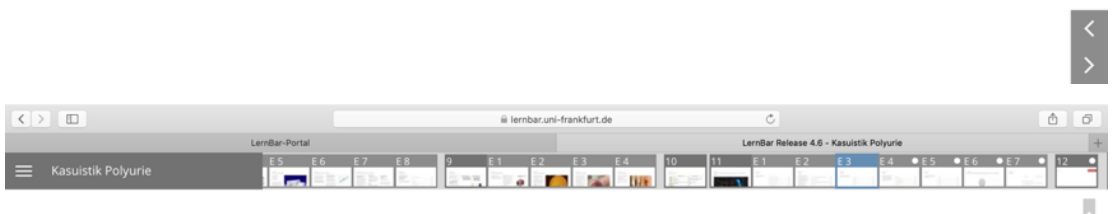

Kasuistik Polyurie

Frage 3

Bei welchem Antidiabetikum besteht am

\begin{tabular}{lc} 
1. Acarbose & 0 \\
\hline 2. Metformin & 0 \\
\hline 3. Insulin lispro & 0 \\
\hline 4. Glibenclamid & 0 \\
\hline 5. Repaglinid & 0
\end{tabular}

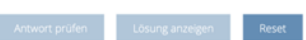




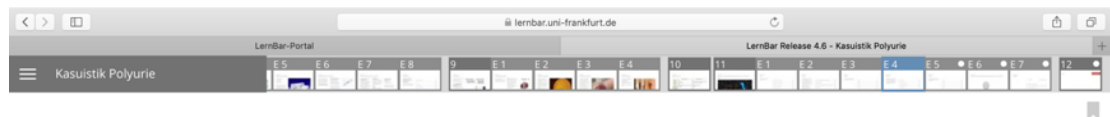

Kasuistik Polyurie

Frage 4

Eine 65- jährige Frau mit einem seit 3

Nachweise ist am besten geeignet nach

dem ersten Zeichen einer diabetischen

\begin{tabular}{lc} 
1. Glukosurie (Stix) & 0 \\
\hline 2. Serumkreatininkonzentration & 0 \\
\hline 3. Mikroalbuminurie (Stix oder Elisa) & 0 \\
\hline 4. Mikrohămaturie (Stix) & 0 \\
\hline $\begin{array}{l}\text { 5. Eine Suche ist nicht notwendig, da nach 3-jahriger Diabetesdauer noch keine } \\
\text { Nephropathie vorliegt. }\end{array}$ & 0
\end{tabular}

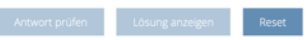

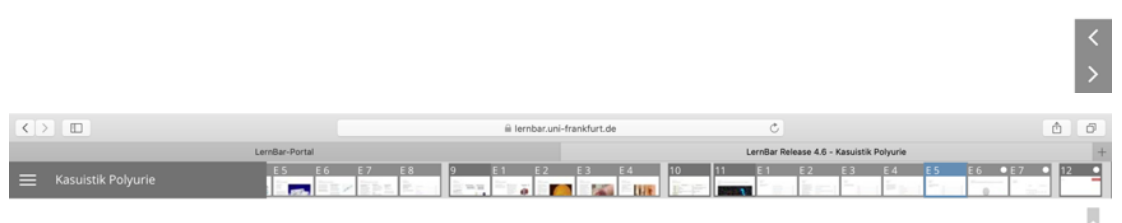

Kasuistik Polyurie

Frage 5

Ab welchem Wert Nüchternblutzucker $(8 \mathrm{~h}$

Diabetes mellitus?

\begin{tabular}{lc} 
1. $116 \mathrm{mg} / \mathrm{dl}$ & $\bigcirc$ \\
\hline 2. $126 \mathrm{mg} / \mathrm{dl}$ & $\bigcirc$ \\
\hline 3. $150 \mathrm{mg} / \mathrm{dl}$ & $\bigcirc$ \\
\hline 4. $200 \mathrm{mg} / \mathrm{dl}$ & 0 \\
\hline 5. $210 \mathrm{mg} / \mathrm{dl}$ & $\bigcirc$
\end{tabular}

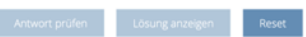

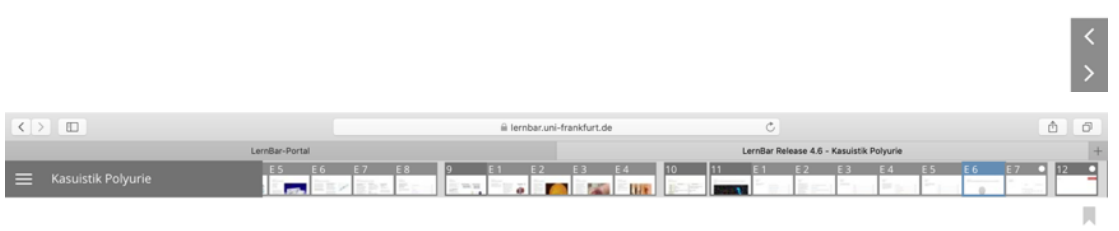

Kasuistik Polyurie

Super! Du hast die Kasuistik geschafft! Bitte evaluiere noch kurz die Kasuistik!

Bild 


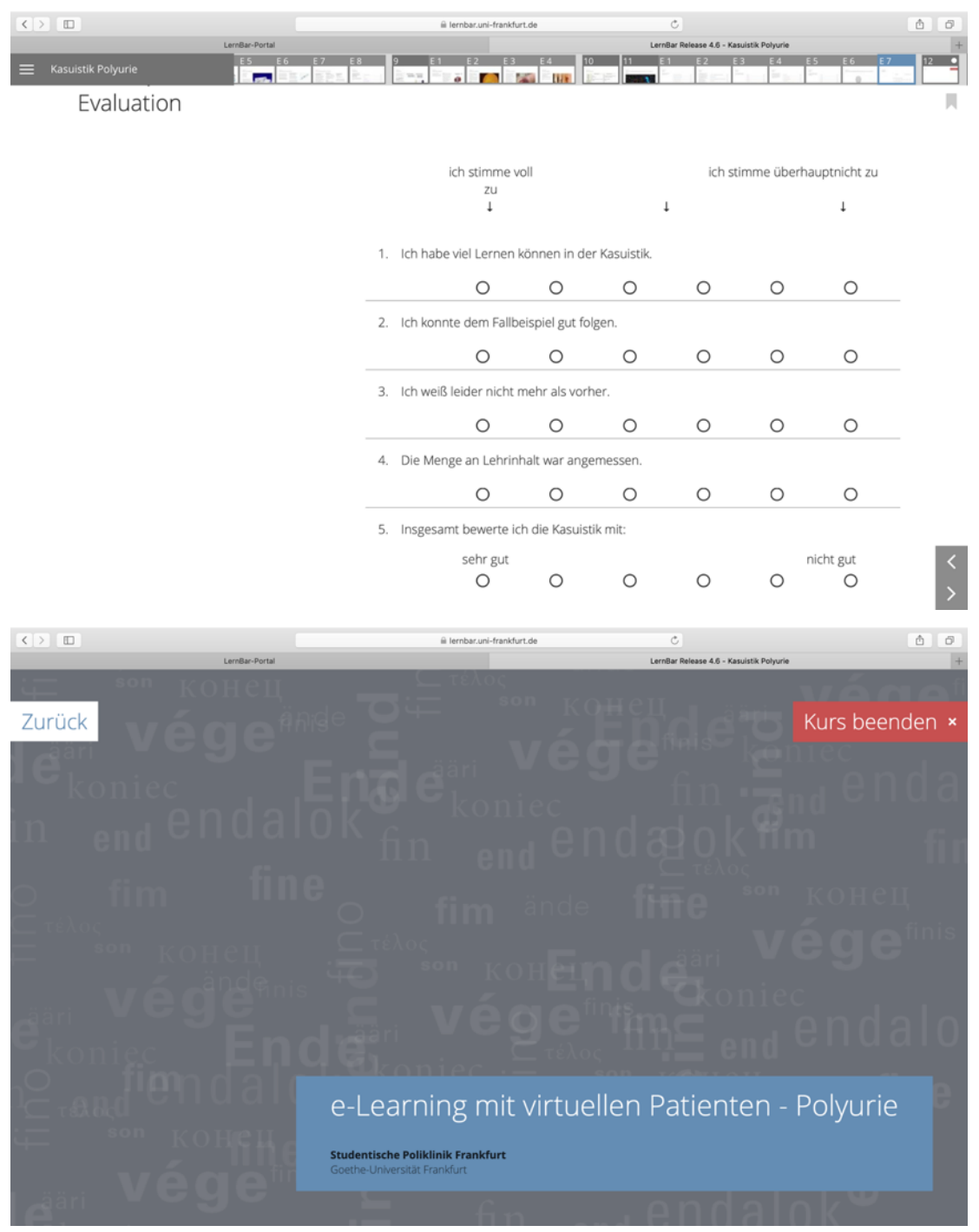


Kasuistik Schwangerschaft

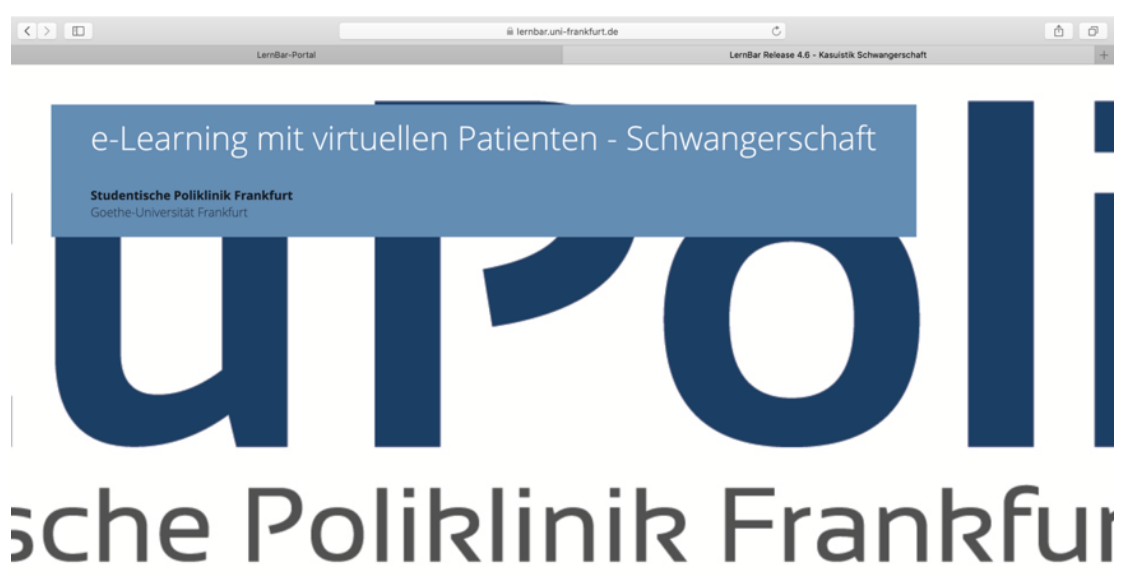

\section{Goethe Universität KURS STARTEN}

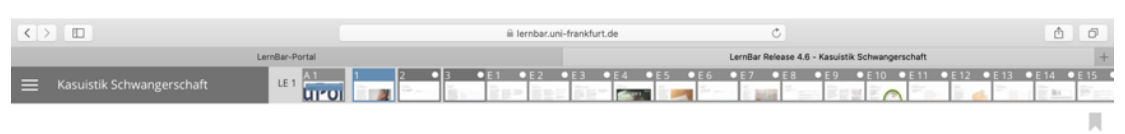

Kasuistik Schwangerschaft

Thema: Schwangerschaft

Hallo und willkommen zur Kasuistik

Schwangerschaft. Die Sprechstunde der

auch schwangere Patientinnen. Wir haben

das Glück, die Schwangeren in die

Sprechstunde einer Gynakologin im

Gesundhetsant uberweisen zu konnen

aber auf unser Wissen über die sind wir.

von Schwangeren angewiesen Daher

findet diese Kasuistik statt Ihr sollt lernen

wie man eine Routine-Untersuchung

durchführt, wie ein Mutterpass aufgebaut

ist und was es fúr Komplikationen geber

Spresotunde genen.

Viel Spaß!

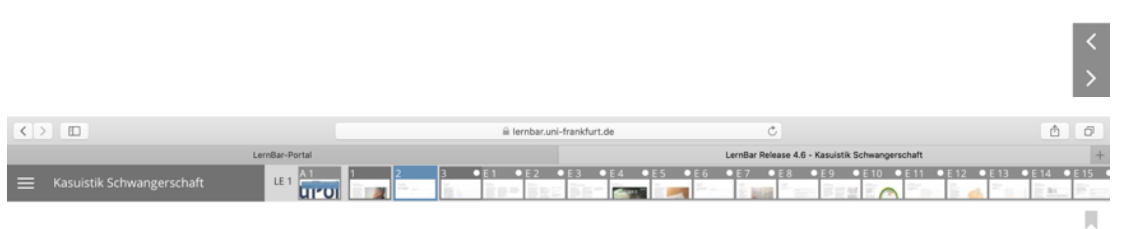

Kasuistik Schwangerschaft

Persönliche Daten

Bitte schreibe in das Feld deinen Vor-und
Namen und deine Matrikelnummer.

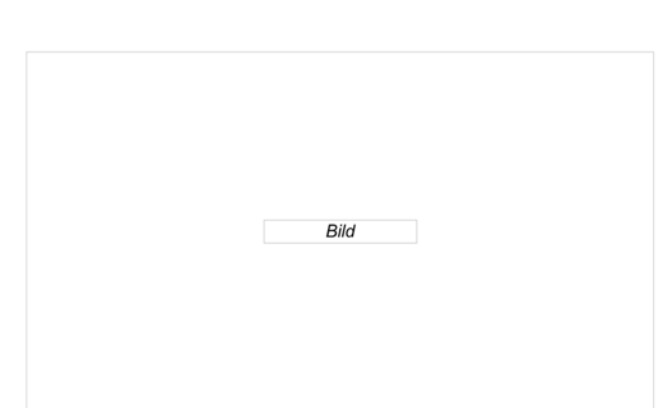

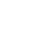




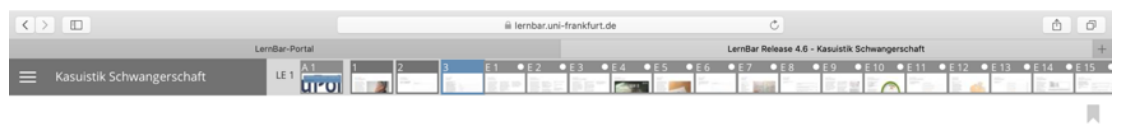

Kasuistik Schwangerschat

Fallbeispiel

Frau P., 27 Jahre alt, kommt zu dir in die

Sprechstunde der StuPoli im

Gesundheitsamt, du kennst sie von zwei

u.a. du und andere Studierende

gemeinsam mit einer kooperierenden
Hebamme betreut habt. Ihre Kinder, 2

und $31 / 2$ Jahre alt sind auch dabei. Auch

die Mutter von Frau P. ist mitgekommen.

Auf die Frage was der Anlass fur den

glaube, erneut schwanger zu sein.

Du musst heute die Erstuntersuchung

übernehmen, da die Hebamme mit einer

Grippe krank geschrieben ist. Du

uberlegst dir was zu einer

Erstuntersuchung einer Schwangeren

Was machst du alles bei der

Erstuntersuchung?

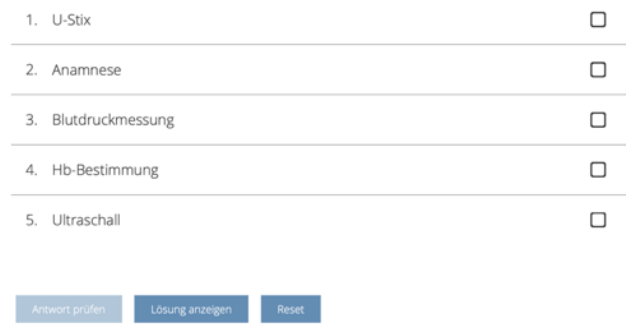

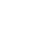

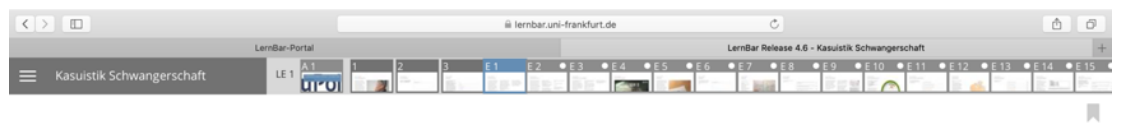

Kasuistik Schwangerschaft

Erstuntersuchung

Wie bei jedem anderen Patienten beginnt man mit einer Anamnese, besonderes

Augenmerk liegt hier bel der familien.

Arbeits- und Sozialanamnese.

Eine gynäkologische Untersuchung

(einschließlich Untersuchung auf eine

Chlamydien-Infektion) sollte ebenfalls

Bestandteil der Erstuntersuchung sein.

Fur eine gute gynakologische

Untersuchung braucht man viel Erfahrung

daher führen wir Studierenden diese in der

Regel nicht durch. Aber man kann schon

mit sehr einfachen diagnostischen Mitteln

schwerwiegende Erkrankungen der

Auf jeden Fall solltest du den Blutdruck messen. Dies ist wichtig (auch bei den möglichst früh Komplikationen wie die schwangerschaftsinduzierte Hyperton restzustellen. Auch das Körpergewicht wird gemessen. Die durchschnittliche

Gewichtszunahme liegt am Ende der

Schwangerschatt bei 10-12kg.

und den Blutzucker.
Weiterhin wird üblicherweise der Urinstatus (Eiweib, Glukose, Infekte) nehmen der Patientin Blut ab. Dies ist neben der $\mathrm{Hb}$ - Bestimmung auch notwendig für den Mutterpass... 


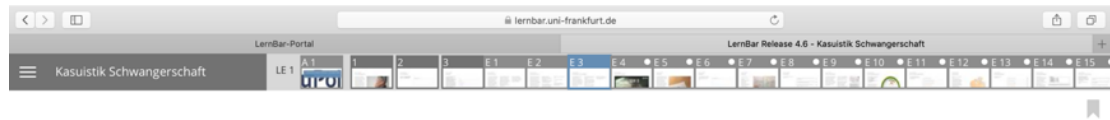

Kasuistik Schwangerschat

Schwanger oder nicht schwanger?

Das Ausbleiben der Periode ist ein sehr

unsicheres Zeichen für eine

Jeichen wären $2 B$ Übelkeit, Erbrecten

möchtest jedoch als sicheres Zeichen ine sonographische Untersuchurg

durchfürnen.

so ist nun doch eine abdominelle

oder Schwindel. Frau P. hatte zwar schon

Sonographie kann Bild sichert

cht, aber

auch der Nachweis von Beta-HCG gilt z.B

Wie Brustspannen oder der sekundaren e Schwangerschaft von Frau P.

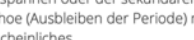

Nachweis fetaler Herzaktion im vaginaler

Uitraschall und ist ab der 5-7. 55 mogrich

p.m... post menstruationem bedeutet de

schwangerschaftszeichen

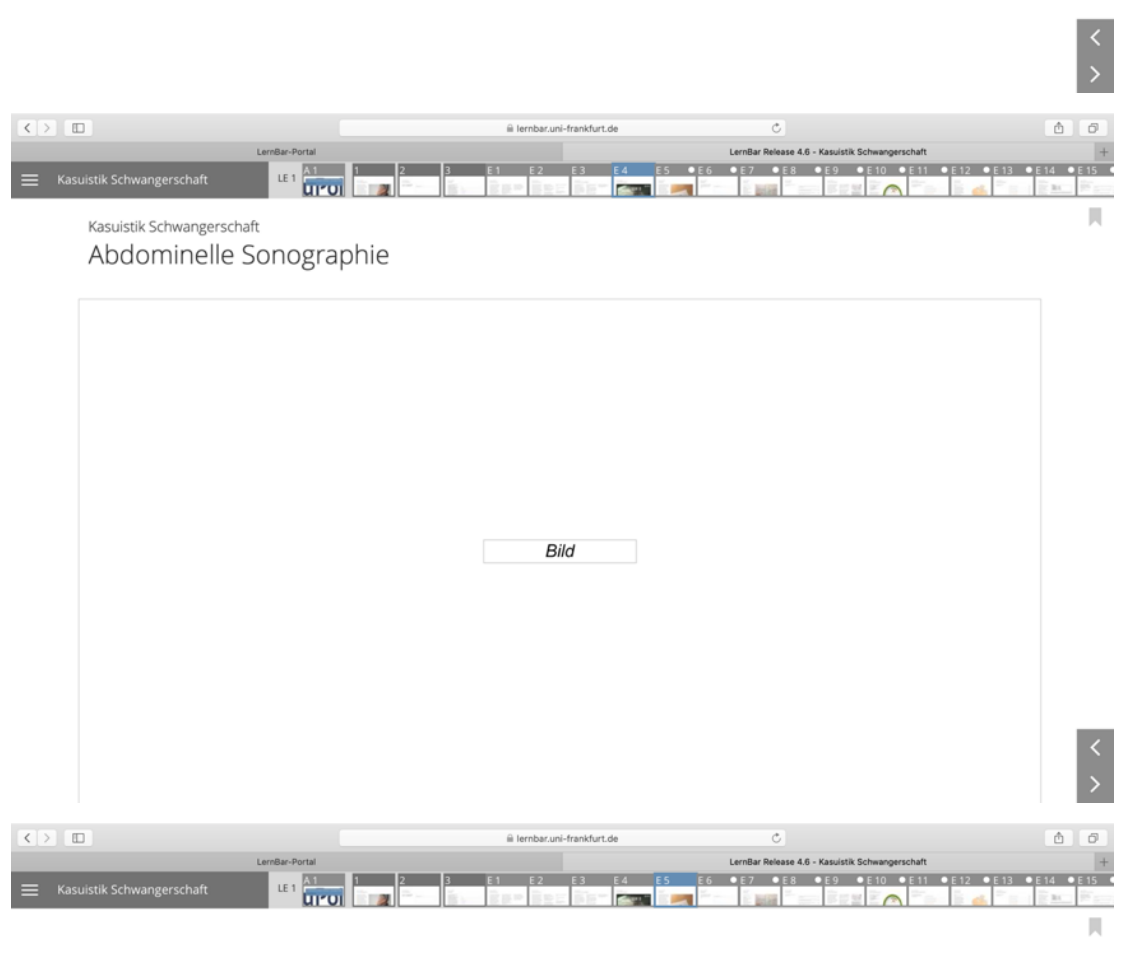

Kasuistik Schwangerschaft

Mutterpass

In der ersten Vorsorgeuntersuchung wird der Mutterpass ausgestell. in intrin werden

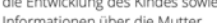
festgehalten Dafür ist es sinnvol, au

Beginn der Schwangerschaft eine

Blutuntersuchung zu machen, auch

Vaginalabstriche auf bestimmte Bakterien, die dem Knd bel der Entbindung schad konnten, werden hier dokumentien.

Mutter immer dabei haben er is auch fis folgende Schwangerschaften

vorgesehen

Wir stellen also den Mutterpass aus.

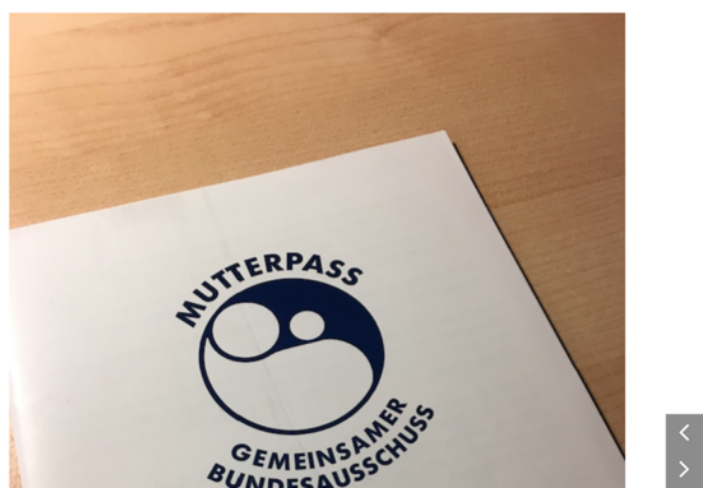




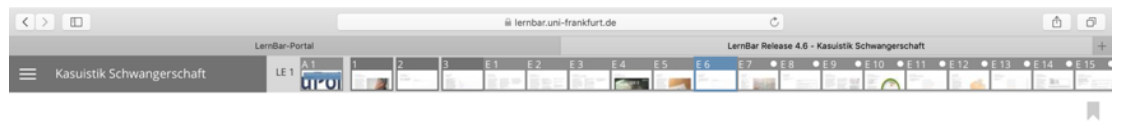

Kasuistik Schwangerschat

Mutterpass

Um den Mutterpass auszustellen, nehmen

bestimmen? Nenne alles, was dir dazy
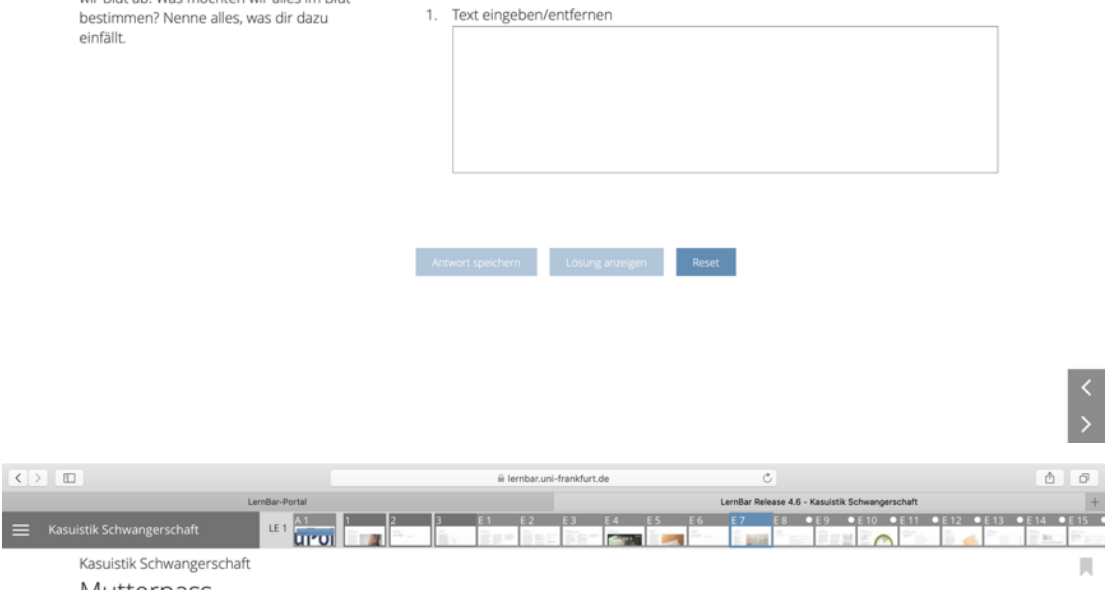

Mutterpass

Wir bestimmen folgendes:

Blutgruppe inkl. Rhesus-Faktor.

irreguläre AK

(ohne dokumentierte

zweimalige Impfung).

Hepatitis B ( $(\mathrm{BBSAg})$

TIV (muss nicht, wird jedoch jeder

Schwangeren empfohien, solitte empfohlen

werden

Toroplasmose-AK \& CMV (bei begründetem

verdacht), TSH, Blutbild

Weitere Infektiologische Diagnostik:

Chlamydien aus Erststrahlurin oder

Abstrich,

ca. 10 Tage vor Entbindung: Abstrich auf $B$.

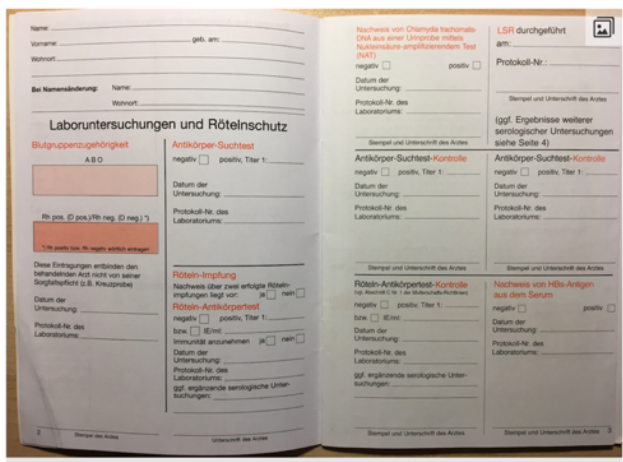

Ergebnisse der serologischen Untersuchungen, die in den Mutterschaftsrichtlinien empfohlen werden, müssen hier eingetragen werder

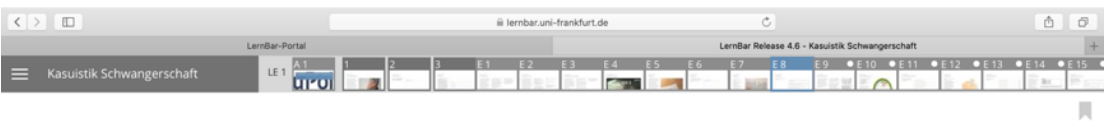

Kasuistik Schwangerschaft

Rhesusfaktor

Was ist der Rhesusfaktor und warum ist

\begin{tabular}{l} 
1. ist ein Oberflächenprotein auf Enythrozyten und spielt nur eine Rolle in der \\
Transfusionsmedizin \\
$\begin{array}{l}\text { 2. ist ein Oberflächenprotein auf Enythrozyten und bei einer Inkompatibilität mit } \\
\text { eine Hämolyse kindlicher Erythrozyten verbunden }\end{array}$ \\
\hline $\begin{array}{l}\text { 3. ist ein Oberflächenrezeptor auf Thrombozyten und wichtig bei eine Rh- negativen } \\
\text { Schwangeren mit Rh- positivem Kind }\end{array}$ \\
$\begin{array}{l}\text { 4. ist ein Oberflachenprotein auf Thrombozyten und kann bei einer Inkompatibilităt } \\
\text { einen Morbus haemolycicus neonatorum bedingen }\end{array}$
\end{tabular}

tin

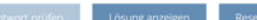




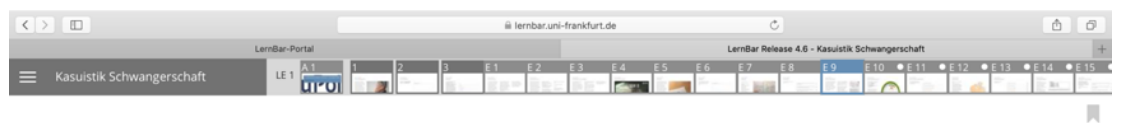

Kasuistik Schwangerschatt

Rhesus-Inkompatibilität

Bei Rh- negativer Mutter und Rh-positivem

Kind (ca. 10 \% aller Schwangerschaften)

positivem Blut des Kindes Rh.lgG.

Antikörper (Anti-D-Antikörper). D

Antikörper werden i.d.R. nach der Geburt

gebildet. Für das erste Kind ist dies som?

kein Problem. Gefannicich wird es erst is

Schwangerschaft).

In Verlauf können die plazentagăngigen Antikörper und in den fetalen Organismus Schwangerschaft mit einem Rh-positive kind).

Es kommt zur hämolycischen Anämie des Feten und es besteht die Gefahr eines Hyarops tetailis. Der Hydrops fetais

flüssigketsane gentulisiente

ungeborenen Kind, die die serösen Höhle (Pleura, Perikard, Peritoneum) sowie die Haut betreffen kann. (Gefahr des Morbus haemolyticus neonatorum (G))

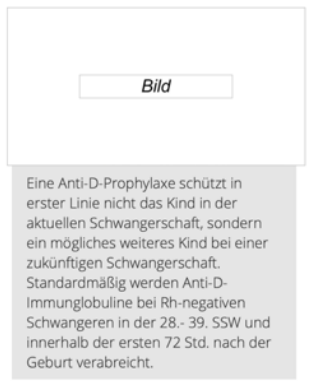

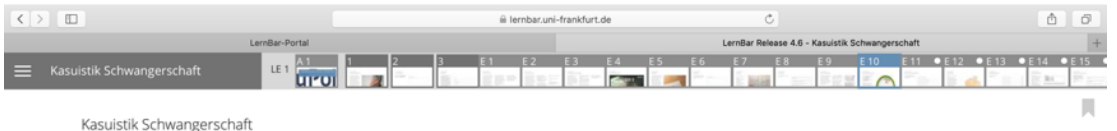

Bestimmung Geburtstermin

Mit der Zyklusanamnese wird der erste Tag Geburtstermin errechnet.

Einfore Naegle Regt:

Letzen Regelbutung +7 Torster Tag der - 3 Monate + 1 Jahr

Frau P. gibt an am 24. November ihre letzte Regelblutung bekommen zu haben. Du schaust nach dem Gebunstermin. Du September rum im nächsten labr

Das sogenannte Gestationsalter entspricht

der Schwangerschattsdauer und wird

berechnet vom 1. Tag der letzten

Regelblutung bis zur Geburt des Kindes

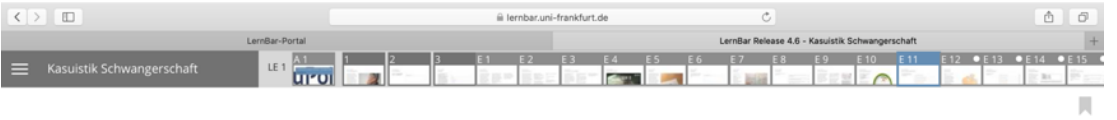

Kasuistik Schwangerschaft

Bestimmung des Gestationsalter

Die Bestimmung des Gestationsalters in

mittels.

\begin{tabular}{lc} 
1. Messung des Beta-HCG-Spiegels & 0 \\
\hline 2. Bestimmung der fetalen Scheitel-SteiB-Lannge & 0 \\
\hline 3. Berechnung der letzen Menstruationsblutung & 0 \\
\hline 4. Feststellung von fetalen Herzaktionen & 0 \\
\hline 5. Gravidarium & 0
\end{tabular}

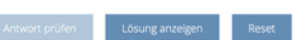




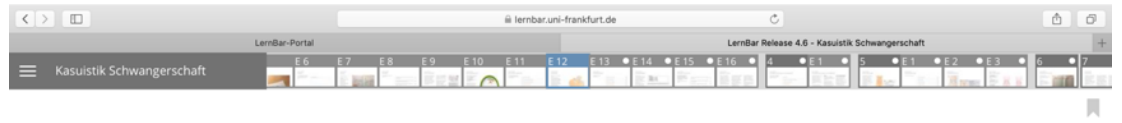

Kasuistik Schwangerschat

Gestationsalter

Bestimmung der fetalen Scheitel-Steiß-

Lănge:

Die sonographische Erhebung

en Screenings gibt

Rückschlüsse auf den Entwicklungsstan

des Kindes und auch auf mögliche

Fehlentwicklungen. Die Scheitel-Steils.

Lange (SSL) ist dabei zentraler Parameter

sowie des Gestationsalters.

Dies ist die sicherste Methode das

Gestationsalter zu bestimmen.
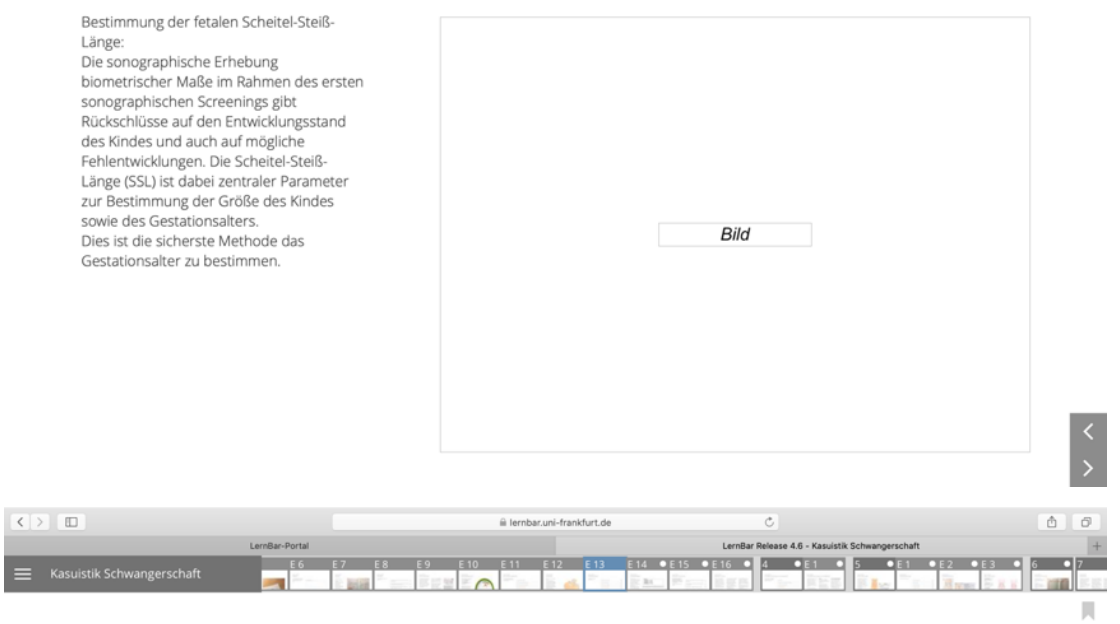

Kasuistik Schwangerschaft

Schwangerschaftsdauer

Wie lange dauert eine reguläre
Schwangerschaft?

\begin{tabular}{ll} 
1. 38 Wochen post menstruationem & $\square$ \\
\hline 2. 38 Wochen post conceptionem & $\square$ \\
\hline 3. 40 Wochen post menstruationem & $\square$ \\
\hline 4. 40 Wochen post conceptionem & $\square$ \\
\hline 5. 42 Wochen post menstruationem & $\square$
\end{tabular}

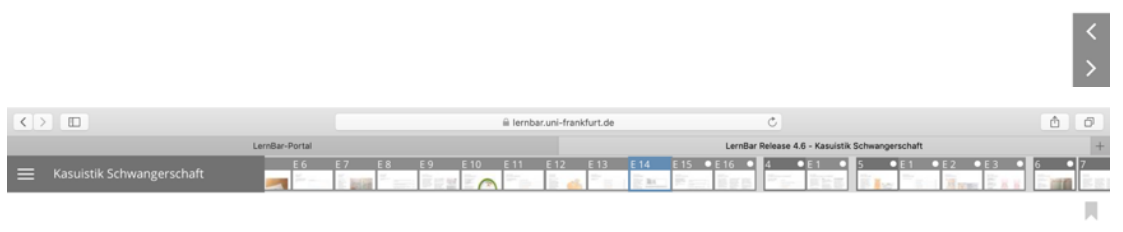

Kasuistik Schwangerschaft

Schwangerschaftsdauer

Super, das ist richtig

Eine reguläre Schwangerschaft dauert 40

Wochen post menstruationem (p.m.) bru.

In der Geburtshilfe arbeitet man

üblicherweise mit dem den post

menstruationem Wochen. Notiert werden

die abgeschlossenen

Schwangerschaftswochen + die

abgeschlossenen Tage (0-6) der gerade

Wenn die Patientin sich zum Beispiel am

Tag ihrer 25. Schwangerschaftswoche

(SSW) befindet, wird dies folgendermaBen

notiert: $24+5$ SSW

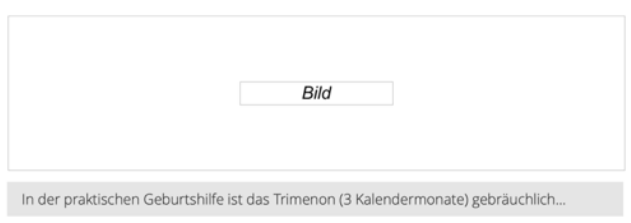

In der praktischen Geburtshilfe ist das Trimenon (3 Kalendermonate) gebräuchlich... 


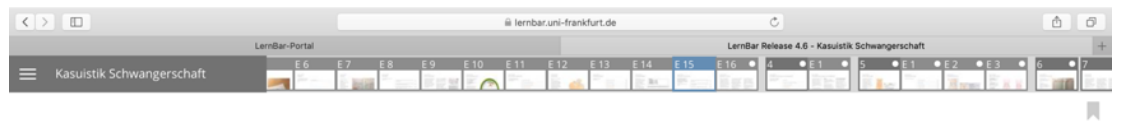

Kasuistik Schwangerschaft

Weiter gehts mit der Beratung

Nach Bestätigung der Schwangerschaft

fahrst du nun ein ausführliches

mit Frau P., bei dem auch die Ernährung

zur Sprache kommt.

Was empfiehlst du deiner Patientin am Infektionsgefahr

2. Reduzierung von Milch und milchhaltigen Produkten insgesamt wegen des hohen $O$

3. ballaststoffarme Ennăhrung wegen der verminderten Darmmotilităt

4. allgemeine Steigerung der Nahrungszufuhr, vor allem im letzten Trimenon um ca. $\mathrm{O}$

5. eiweiBarme Mischkost zur Reduzierung des Gestoserisikos

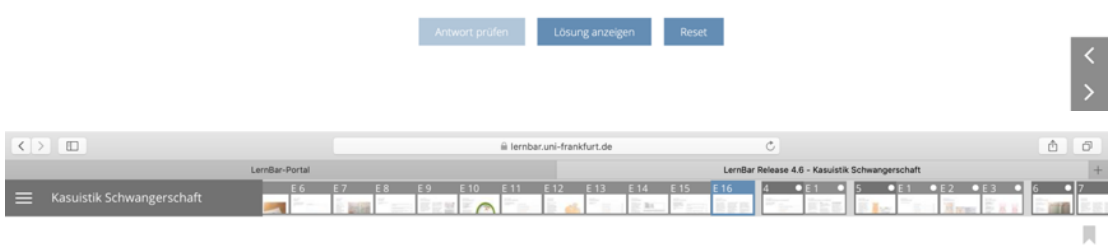

Kasuistik Schwangerschaft

Beratung zur Ernährung

Der Verzehr von Rohmilchprodukten

(Rohmilchkase, unpasteurisierte

Infektionsgefahr für Listerien einoher

Schwangere haben an sich schon ein 12 .

fach erhöhtes Risiko für eine Infektion.

zudem können Listerien (im Gegensatz z

vielen anderen Bakterien) die

Plazentaschranke uberwinden und das

Schwangere haben einen erhöhten

Füssigkeitsbedarf. Sie sollten mindestens

2-3//Tag trinken, am besten Wasser oder

ungesüß̈ten Tee. Stark zuckerhaltige

Vor allem am Beginn der Schwangerschaft solte die werdende Mutter Folsäuren

Mengen zu sich nehmen da diese

spurenelemente wichtig in der fetale

Entwicklung sind. Folsäure findet sich v.a. in

Gemüse, Vollkorn und Obst.

jod kann durch jodiertes Speisesalz

a genommen werden. Zusätzlich können

eingenommen werden. In der

schwangerschaft sollte kein grüner Tee

getrunken werden, weil er die Aufnahme

von Folsăure hemmt
Viele Schwangere entwickeln einen

Esisenmangel. Die versorgung mit Ess

wird daher auch im Mutterpass

Vegetarierinnen. Fisen kann gut dut sind

Fleisch und grünes Gemüse aufgenommen

werden.

ür die Entwicklung des kindlichen Gehirns

ist eine gute versorgung mit Omega-3.

Schwangere sind

en zu bekommen und

diese auf das Kind zu übertragen, Deshalb

soll Obst und Gemüse nur mit Trinkwasser

gewaschen werden. Der Verzehr von

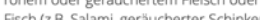

Fisch (2.B. Salami, geraucherter Schinken,

sie den Parasiten Toxoplasmose enthalten

können.

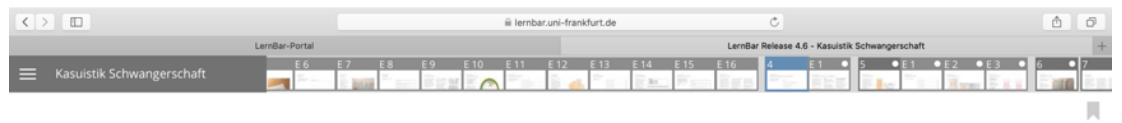

Kasuistik Schwangerschaft

Physiologische Veränderungen in der Schwangerschaft

Bei einer normalen Gravidităt gehōrt zu

Organismus am wenigsten:

\begin{tabular}{lc} 
1. Hyperventilation & 0 \\
\hline 2. Ausbildung von Varizen im Bereich von Vulva und Vagina & 0 \\
\hline 3. Erhöhung des Herzminutenvolumens & 0 \\
\hline 4. Vermehrung der Bluttipide & 0 \\
\hline 5. Diarrhö & 0
\end{tabular}

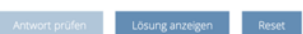




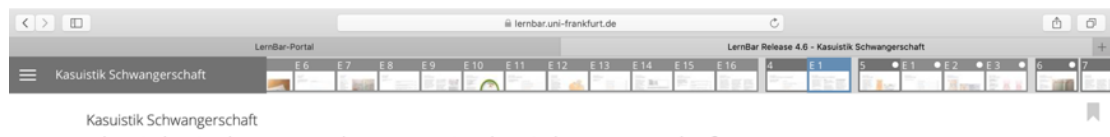

Kasuistik Schwangerschaft

Physiologische Veränderungen in der Schwangerschaft

Super! Zur Diarrhoe kommt es nicht. Es ist

praktisch das Gegenteil der Fall:

Progesteron fuhrt zu einer Senkung der

Darmmotilităt, einer vermehrten

mechanischen Kompression durch der

Uterus. Demzufolge ist bei einer

Schwangerschaft eher mit Verstopfung zu

rechnen

Hier noch mal zusammengefasst, zu

welchen physiologischen veranderung es

bei einer Schwangeren komm

Herz- und Kreislauf:

Der periphere Gefäßwiderstand sink

Tonus der glatten Muskyirung wird der

Dies hat zur Folge, dass die Herzfrequenz

und das Herzminutenvolumen zunehmen.

Durch den erniedrigten peripheren
Gefäßwiderstand sinks der arterielle

Mitteldruck
Atmung:

DeVentilation steigt, Schwangere

typerventilieren physiologischerweise. Der Sauerstoffverbrauch steigt. Dyspnoe ist ein ges symptom

Insgesamt hat die Schwangere ein größeres Blutvolumen, auch dies wird durch den

verussacht (RAAS. Aktivierung vermeh

Wasserretention). In einigen Fällen kann

das ein auskultierbares Systolikum

verursachen, oder eine sog

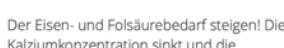

Gesamtplasmaproteine fallen ab.
Vena-Cava-Kompressionssyndrom:

Mit Fortschreiten der Schwangerschaft wird

er in Rückenlagen eine Kompression der V.

cava inferior bewirken kann. Folglich komm

zum Blutoruckabfali, erniedrigtem $\mathrm{H}$.

und eine Abnahme der uterinen

va in der Spatschwangerschaft solte

daher immer in Linksseitenlage gelagert

werden

GIT:

Verstarkte Übelkeit (Gewichtsverlust),

GIT (Sodbrennen Obstipation)

Wasser- und Elektrolythaushalt

Das Gesamtkorperwasser steigt

(Knöchelödeme, generalisierte Odeme).

Fließgeschwindigkeit des Urins vermindert

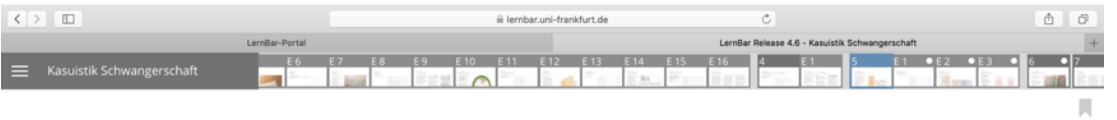

Kasuistik Schwangerschaft

Weitere Untersuchungen

Du verabschiedest dich nun von der

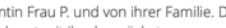

veretersuchungstermin, der mit

Hebamme stattinden wird. Bestandte.

der Untersuchungen sind dann:

Anamnese (gab es Veranderungen und neu

aufgetretene Probleme?), Bestimmung des

che Tastuntersuchung).

Gewichtbestimmung
Blutdruckmessung,

Urinuntersuchung auf Zucker, Eiweiß und

Blutbild zur Kontrolle des $\mathrm{Hb}$

In den Mutterschaftsrichtilinien sind

insgesamt 3 sonographische Screening

Untersuchungen vorgesenen

SSW p.m.

Bild

Hier siehst du veranschaulicht den Fundusstand je nach Schwangerschaftswoche (SSW).

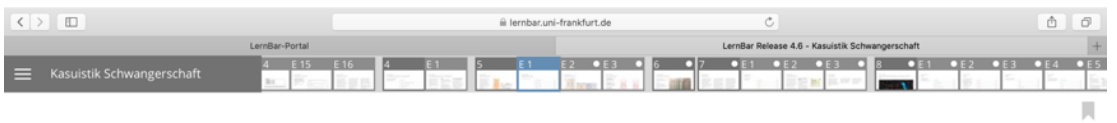

Kasuistik Schwangerschaft

Fundusstand-Bestimmung

Wie bestimmt man den Fundusstand

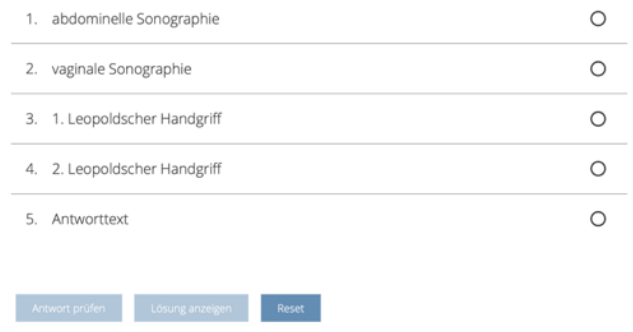




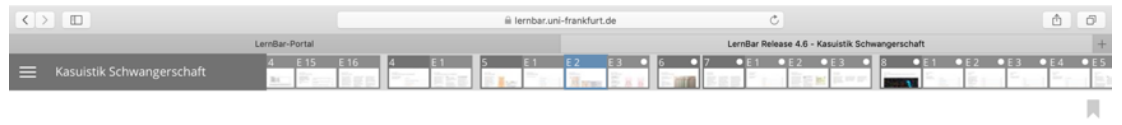

Kasuistik Schwangerschaft

Fundusstand-Bestimmung
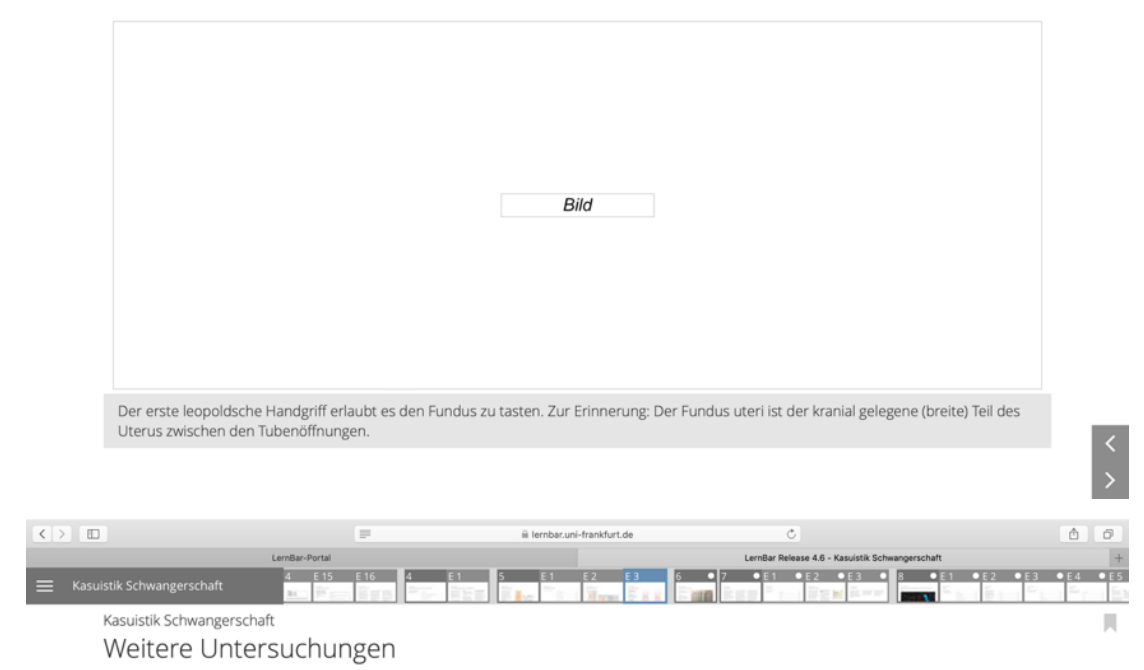

1. Leopold-Handgriff: Beidhändige Prüfung der kindlichen Lage
ISchragg-/Querlage) und

ISchrag-/Querlage) und des Fundusstands

2. Leopold-Handgriff: Beidhăndige

Prüfung der Stellung des kindlichen
Rückens (Rücken kann zur rechten oder

linken Seite der Mutter zeigen)

3. Leopold-Handgriff

Eine Hand tastet oberhalb der Symphys

nach dem vorangehenden Kindstell. Er

Schädel- oder Steißlagell!!

4. Leopold-Handgriff: Ermittliung des

Hohenstands des vorangehenden

Kindsteils mit beiden Händen (erst in

späterer Schwangerschaft,

Die Leopoldschen Handgriffe sind jedoch

sich die Kindslage noch multiple verändern

nehmen wird.

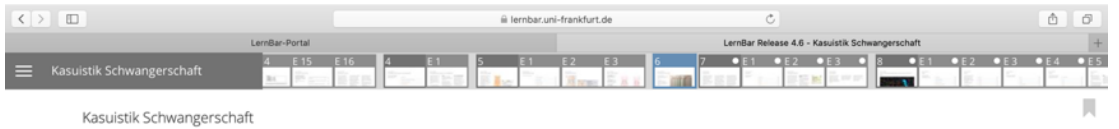

Kasuistik Schwangerschaft

Der nächste Termin für Frau P.

Nun, nachdem Frau P. ihre

Erstuntersuchung hat, vereinbart ihr einen

Termin in 4 Wochen zur erneuten

Kontrolle.

Bis 30. SSW p.m.: Vorsorgeuntersuchungen

sollten im Abstand von 4 Wochen

50.55 p

.

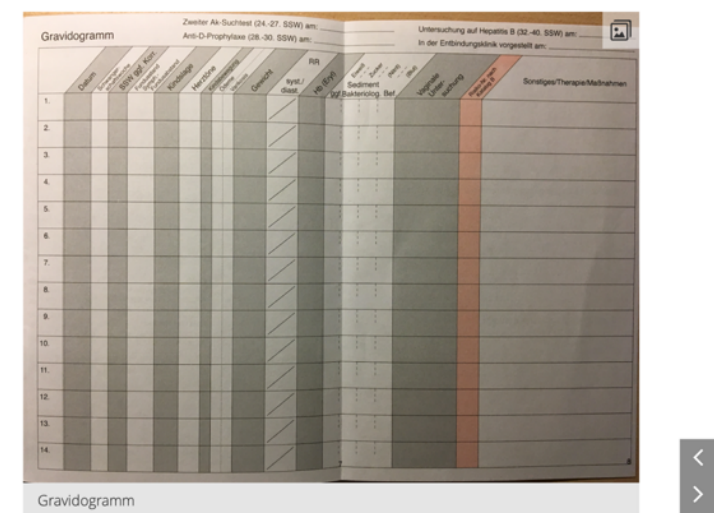




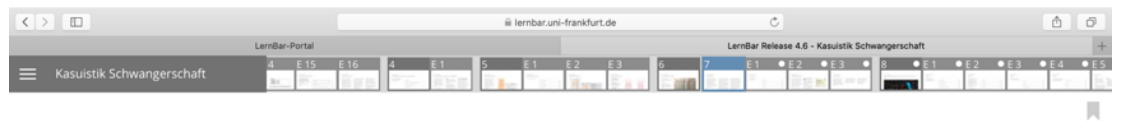

Kasuistik Schwangerschaft

Komplikationen

Komplikationen in der Schwangerschaft sind möglicherweise schon früh in den Daher ist es wichtig auch immer an

pathologische Verläufe in der

Schwangerschaft zu denken.

Es gibt zahlreiche pathologische verlauf

wir schauen uns hier zwei ausgewähtte

mpsie und HELLP. sist eine gewisse Zeit vergangen und $\mathrm{Fram}$ P. befindet sich ca. in der 30 . SSW. Die etzten Termine konnte sie alle nich

rechisseitize Fau P. entwickett

Ubelket und ausserpraster Mattigkeit. Da sie dies zunächst fur einen „verdorbenen Magen" halt, versucht sie, ihre

Beschwerden mit Kamillentee zu kurieren. zunehmen und sie zudem in an ster 30 . schwanger ist, entschließt sie sich am Nachmittag, den hausärrtlichen Notdienst

aufzusuchen, um jedes Risiko zu

Nach ihren Angaben und dem Eintrag in bistlon hoss bestent ene Gravidităt, die der körperlichen Untersuchung finden sich ein Druckschmerz im rechten Oberbauch, insbesondere bei tiefer Inspiration und sehr lebhafte. Der Blutdruck betrag 165/110 mming. Im Urin-Streifentest

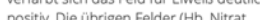

Leukozyten, Nitrit, Glukose) zeigen negative

Ergebnisse an.

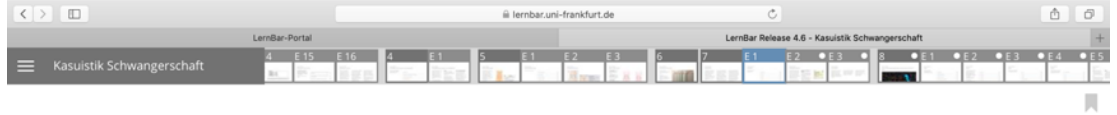

Kasuistik Schwangerschat

Komplikation

Welche der Diagnosen ist am

\begin{tabular}{lc} 
1. vorzeitige Wehen & 0 \\
\hline 2. Präeklampsie & 0 \\
\hline 3. Appendizitis & 0 \\
\hline 4. Cholezystitis & 0 \\
\hline 5. infektiöse Gastroenteritis & 0
\end{tabular}

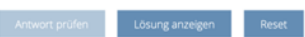

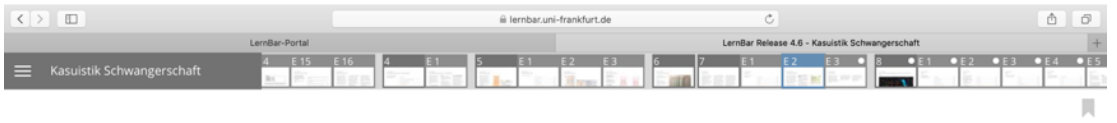

Kasuistik Schwangerschat

Präeklampsie/ Eklampsie

Die beschriebenen Symptome sprechen deutich fur eine Praeklampsie, die durch und Proteinurie (mit oder o hne Odeme) definiert ist. Schmerzen im rechten Oberbauch sind dabei hinweisend auf $\mathrm{d}$ as sogenannte HELLP.Syndrom, während die Hyperreflexie als Zeichen fur eine

beginnende Eklampsie au Werten ist.

Der Verlauf eine Präeklampsie kann von milder Symptomatik bis hin zum Vollbild einer Eklampsie reichen. Ein erhohtes
Risikk für dieses Krankheitsbild tragen vor allem sehr junge und ätere

Erstschwangere, Adipöse, Diabetikerinnen und Schwangere mit Nierenerkrankung

angerschaften ebenfalls

\section{Aggravatio
Eklampsie:} Unwohlsein sind Symptome, die für den Ubergang einer Prëeklampsie zur Eklampsie stehen. s kann zu generalisierten tonisch. Nonischen Krampfanfallen (eklamptischer Ar den Fetus bei plazentare

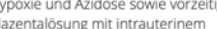
Fruchtod

Präventiv ist dementsprechend wichtig ein zu erkennen Norsorgeuntersuchungen, Anamnese!)

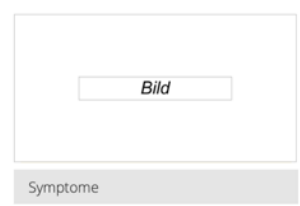

Symptome 


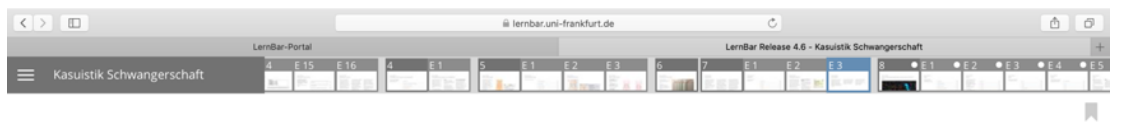

Kasuistik Schwangerschat

HELLP-Syndrom

Der Begriff HELLP- Syndrom bezeichnet

das Vorhandensein einer Hămolyse

(elevated liver enzymes) und einer.

erniedrigten Thrombozytenzahl (low

platelets count) in der Schwangerschaft. Es

der Praeklampsie und weist eine hohe

Wesentlichen sind die Symptome der

chmerz wird verussacht durerc. Der

Mikrothromben in der Leber, die zu einer

Leberschwellung mit einer Kapselspannung

ts somit ein Akronym aus diesen dre

führen

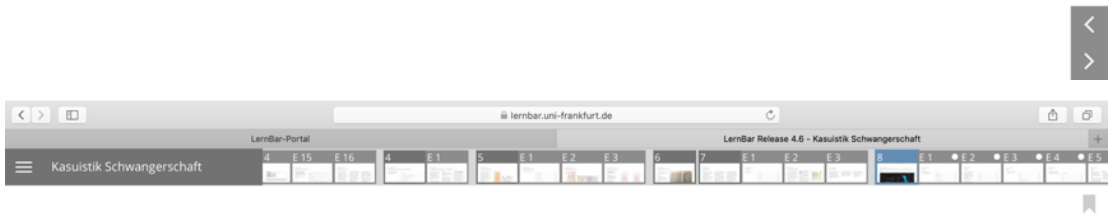

Kasuistik Schwangerschaft

Super, nun ist die Kasuistik abgeschlossen. Zum Schluss noch 5 Fragen zum

üben :)

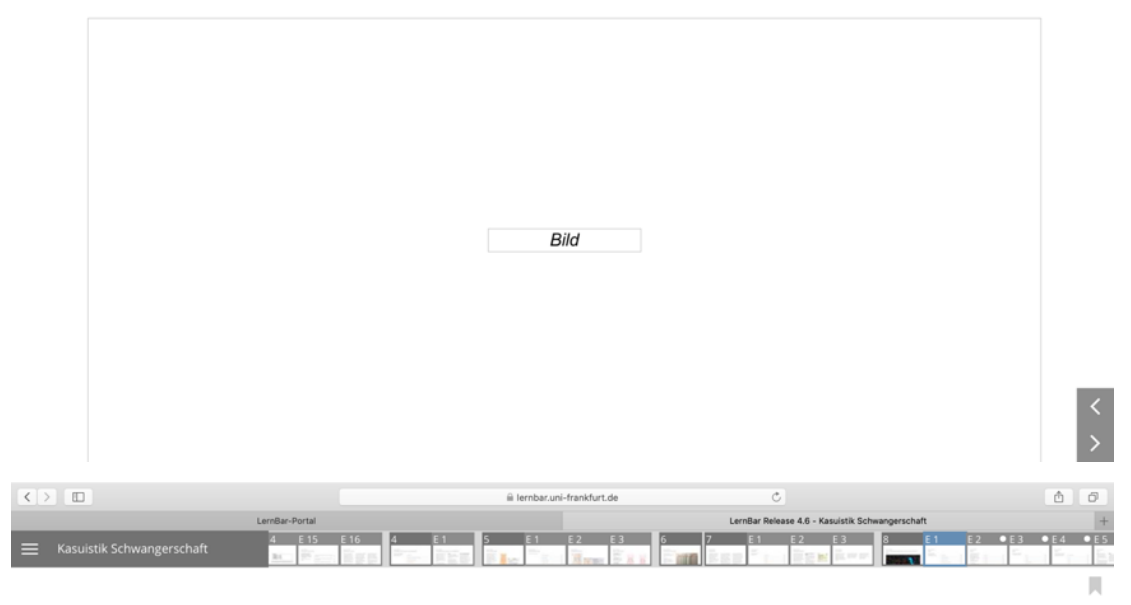

Kasuistik Schwangerschaft

Frage 1

Die häufigste Ursache einer

Schwangerschatsanamiem mit

\begin{tabular}{lc} 
1. ein Eisenmangel & 0 \\
\hline 2. ein latenter Folsăuremangel & 0 \\
\hline 3. ein Vitamin-B-12-Mangel & 0 \\
\hline 4. ein erhöhter Folsäurebedarf des Fetus & 0 \\
\hline 5. eine verminderte Eisenresorption im Darm & 0
\end{tabular}

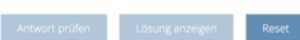




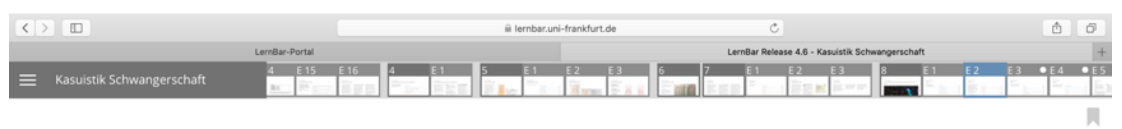

Kasuistik Schwangerschat

Frage 2

Die Geburt eines Kindes, hier mal Peter

genannt, erfolgte am errechneten

gemäß der Naegele-Regel unter -

Zugrundelegung folgender Angaben: In

welchem Zeitrraum war - bei Annahme

eines ungestorten

Schwangerschaftsverlaufes - Peters Gebur

Zykluslänge: 28 Tage

1. Tag der letzten Regelblutung: 5. Mărz

Konzeption: 18. Mărz

\begin{tabular}{lc} 
1. $10 .-12$. November & 0 \\
\hline 2. 24.-26. November & 0 \\
\hline 3. 1.-3. Dezember & 0 \\
\hline 4. 10.12. Dezember & 0 \\
\hline 5. 24.-26. Dezember & 0
\end{tabular}

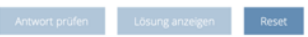

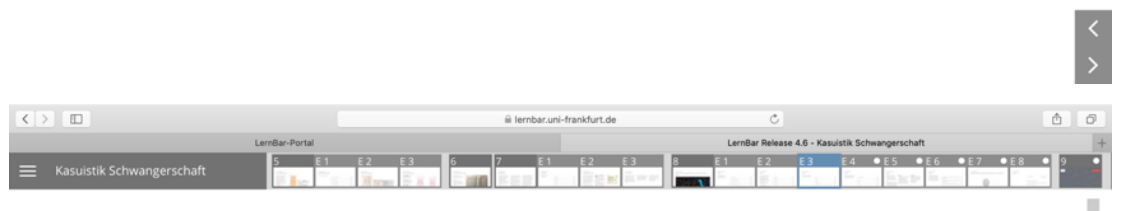

Kasuistik Schwangerschaft

Frage 3

Welche der folgenden Symptome passt

\begin{tabular}{lc} 
1. Oligurie & 0 \\
\hline 2. Proteinurie & 0 \\
\hline 3. Hypertonie & 0 \\
\hline 4. starke Gewichtsabnahme & 0 \\
\hline 5. ggf. Odeme & 0
\end{tabular}

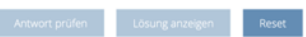

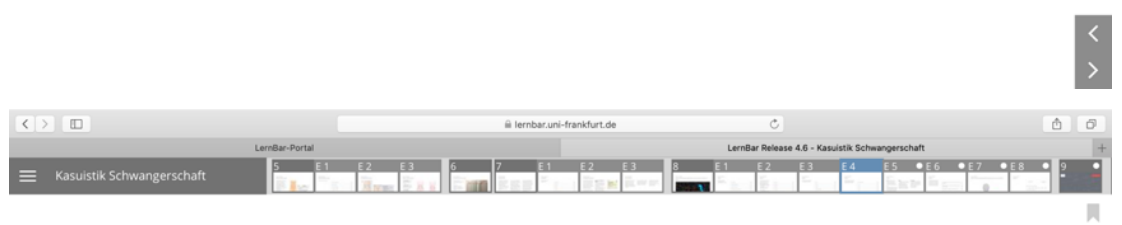

Kasuistik Schwangerschaft

Frage 4

Welche serologischen Untersuchungen

härif druch scefinnt baw angeboten?

\begin{tabular}{ll} 
1. Lues Suchtest (TPHA) & 0 \\
\hline 2. HIV-AK & 0 \\
\hline 3. HSV-2PCR & 0 \\
\hline 4. CMV-AK & 0 \\
\hline 5. HBSAG & 0
\end{tabular}

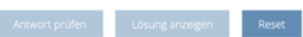




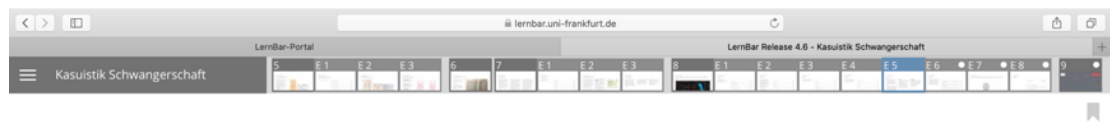

Kasuistik Schwangerschat

Exkurs

CMV: Mikrocephalus, zerebrale

Verkaikungen, Hydrozephalus, Anäm

Schwangerschaft bei Erstinfektion der

Mutter auf das Kind übertragen werden.

$\begin{array}{ll}\text { HIV: } & \text { entwickeln eine Leberzirrhose. Dur } \\ \text { passive \& aktive Impfung des }\end{array}$

Im Gegensatz zu Erwachsenen, bei denen

u beobachten ist, ist dies bei

Die Treponemen sind plazentagăngig und

Frühgeburt führen. Bei einer Manifestation

in der spaten Schwangerschaft führt die

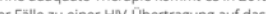

der falle zu einer Hiv-Ubertragung au das

Infektion zu Keratitis,

$\begin{array}{ll}\text { psssive \& aktive Impfung des } & \text { Innenohrschwerhörigkeit und } \\ \text { Neugeborenen kann eine Ubertragung } & \text { Gesichtsfehlibildungen }\end{array}$

und optimale Therapie zu keiner Mutter.

Geburt nertindert werde

Kind Ubertragung

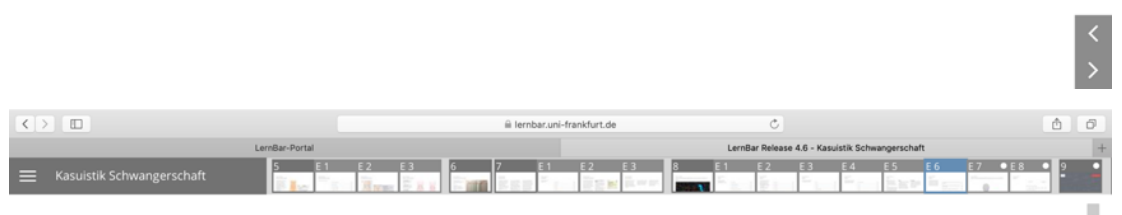

Kasuistik Schwangerschaft

Frage 5

Nach Bestätigung der Schwangerschaft

funnt der fravenarze ein austuritiches

mit Frau B, bei dem auch die Ernöhronang

zur Sprache kommt Was empfieht der

1. Verzicht auf Rohmilchprodukte, auch auf Weichkäse aus Rohmilch wegen der Infektionsgefahr

2. Reduzierung von Milch und milchhaltigen Produkten insgesamt wegen des hohen Kalziumgehaltes

3. ballaststoffarme Ernährung wegen der verminderten Darmmotilităt $\quad$

4. allgemeine Steigerung der Nahrungszufuhr, vor allem im letzten Trimenon um ca.

eiweißarme Mischkost zur Reduzierung des Gestoserisikos

0

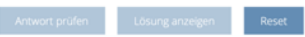

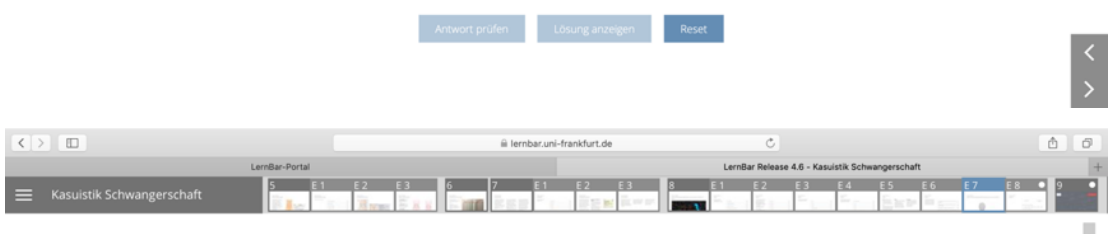

Kasuistik Schwangerschaft

Super! Du hast die Kasuistik geschafft! Bitte evaluiere noch kurz die Kasuistik!

Bild 


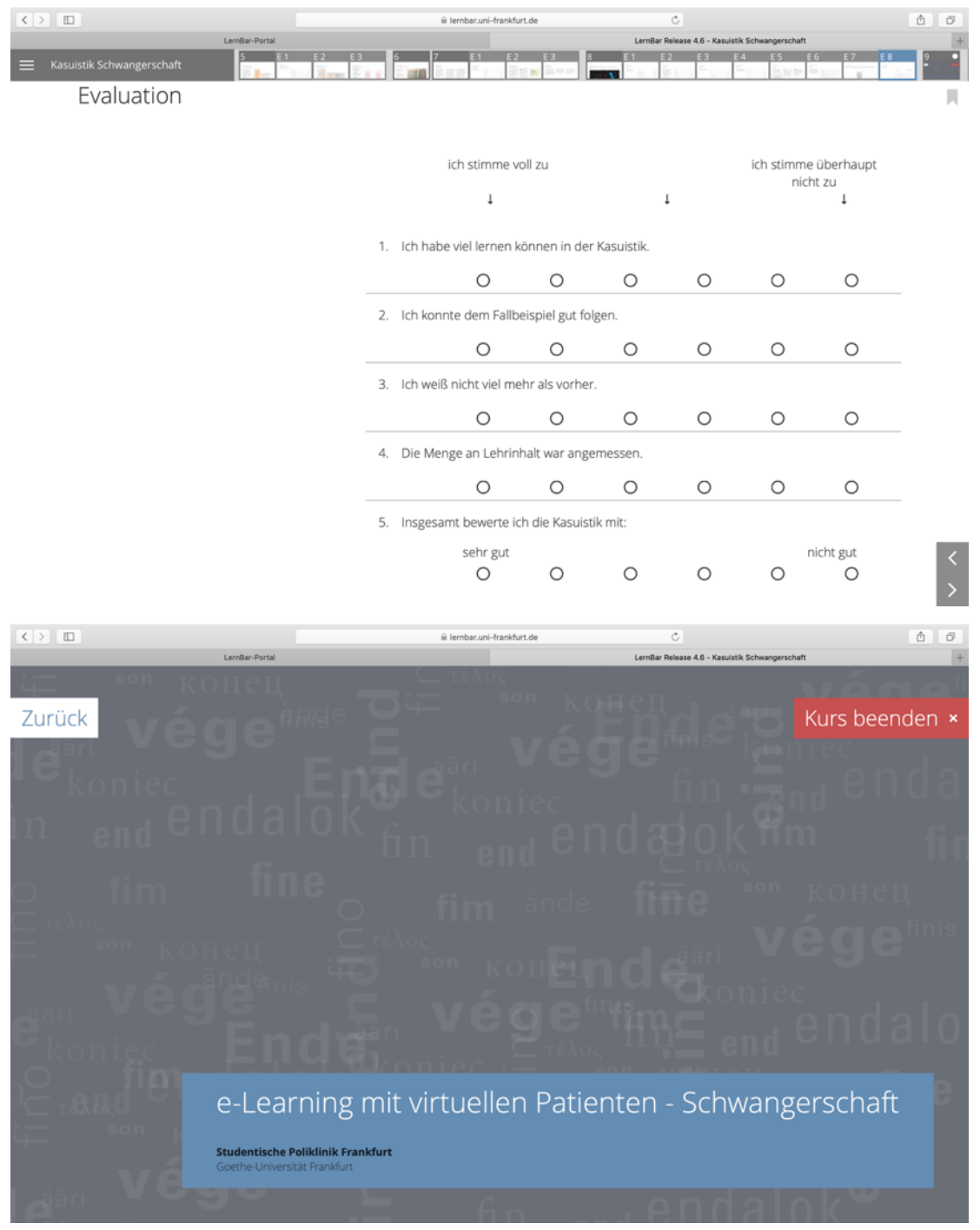


Kasuistik Arterielle Hypertonie

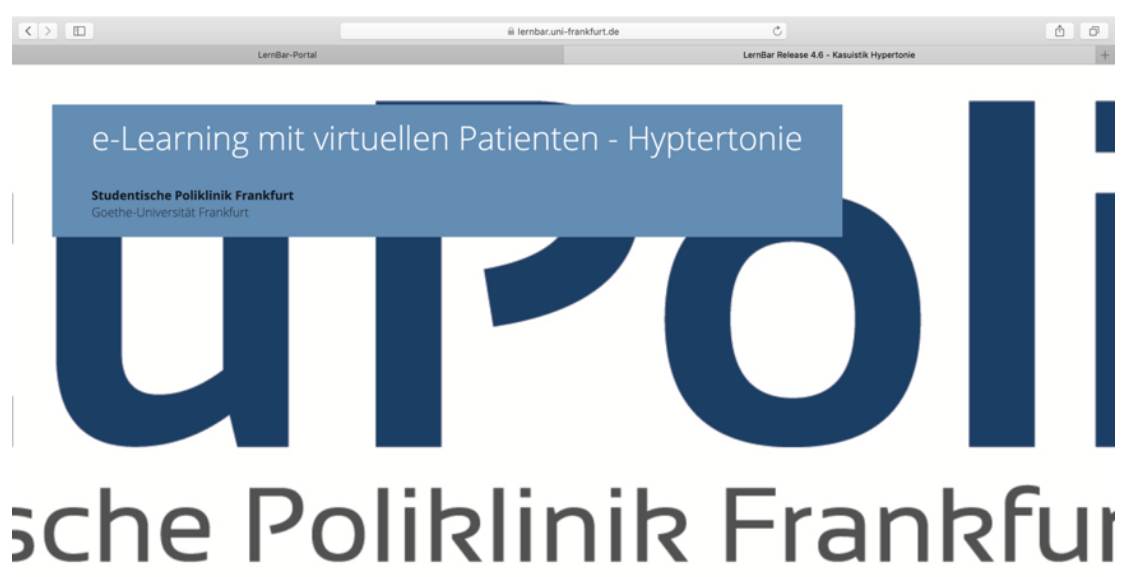

\section{Goethe Universität KURS STARTEN}

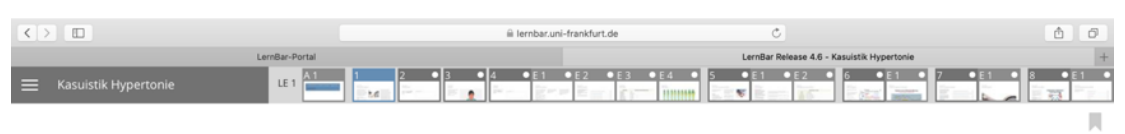

Kasuistik Hypertonie

Thema: Arterielle Hypertonie

Hallo, in dieser Online- Kasuistik wirst du

dich mit der arteriellen Hypertonie

Fallbeispiel geben. Mit Informationstexten,

Bildern und Fragen wirst du dich durch das

Thema arbeiten und am Ende dieser
Kasuistik folgendes gelernt haben:

Diagnostik der Hypertonie

Diagnostik der Hypertonie
Primärer vs. sekundărer Hypertonus und

Häufigkeit

Risikofaktoren für primären Hypertonus

Ursachen sekundärer Bluthochdruck

Einteilung Schweregrade Hypertonie

Hypertensive Krise vs. Notfall

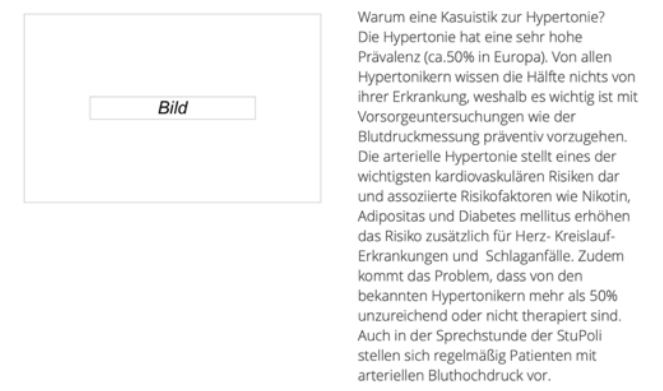

De mene Kasuistik zur Hypertonie?

Prävalenz (ca.50\% in Europa) Von a

Hypertonikern wissen die Halfte nichts von

Vorsorgeuntersuchungen wie der

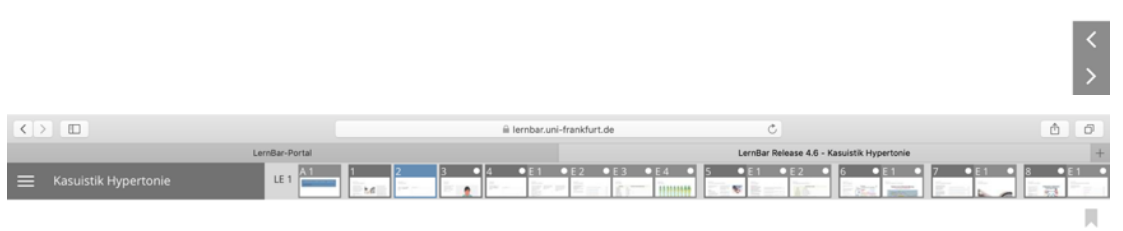

Kasuistik Hyperton

Persönliche Daten

Bitte schreibe in das Feld deinen Vor-und

VTipp

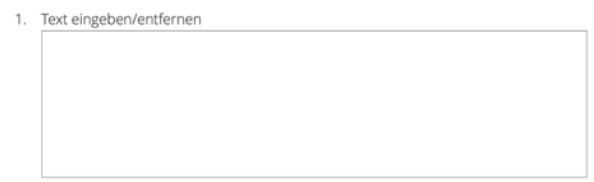




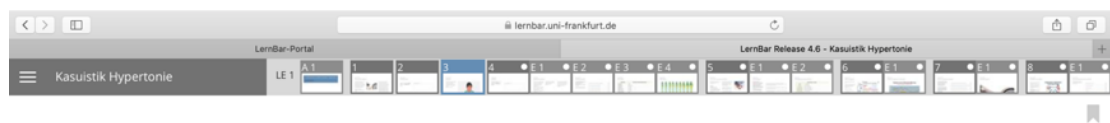

Kasuistik Hyperton

Fallbeispiel

Der 50- jahrige Herr J.B. kommt (mit rotem

Kopf zu dir in die Allgemeinarzt. Praxis

weil er seit geraumer Zeit über

morgendliche Kopfschmerzen klagt und

erhöhte RR. Werte beim Blutspenden

aufweist.

Du erhebst eine Anamnese.

Bild

LE 13

Kasuistik Hypertonie

Anamnese

Neben allgemeinen Fragen, ist es wichtig

Ans du bestimmte fragen in der

Herrn J.B.?

1. Text eingeben/entferner

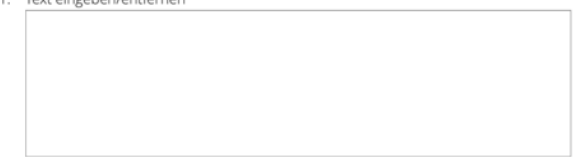

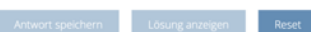

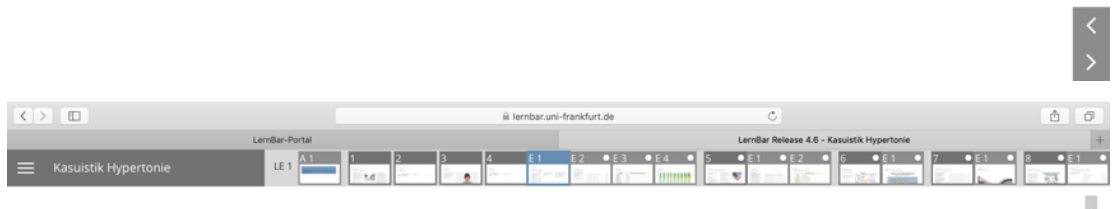

Kasuistik Hypertonie

Ergebnisse der Anamnese

Du erfährst, dass Herr J.B. 50 Jahre alt ist

$176 \mathrm{~cm}$ gro 13 ist und $94 \mathrm{~kg}$ wiegt. Er $\mathrm{k}$ agt

Außerdem erklärt er. dass er öters

An Medikamenten nimmt der Patient

Zu einer gründlichen Anamnese gehört

Herr dB b leidet an nannese: Der Vater

Herzzcrankheit) und hatte einen Bypass. Der

erhöhte Blutdruck-Werte beim

Herzkrankheit) und hatte einen Bypass,
Großvater starb an einem Schlaganfall.

Da du nach Vorerkrankungen gefragt hast,

weilst du auch, dass Herr J.B. Diabetes

mellitus Typ 2 und eine

政

lahren (30 py) und trinkt gelegentlich ein

Glas Bier oder Wein. 


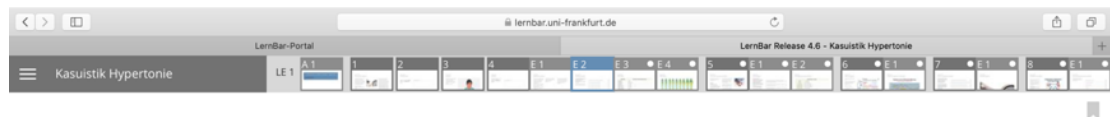

kasuistik Hyperton

Body Mass Index (BMI)

Du kannst den BMI deines Patienten wie

folgt errechnen: $B$ MI = Gewicht

Hier noch mal die Werte vom Patienten:

$176 \mathrm{~cm}, 94 \mathrm{~kg}$

Was hat der Patient nun für einen BM!

2. BMI von 53.4 dieser Wert liegt im Normbereich

$\left(\mathrm{kg} / \mathrm{m}^{\wedge} 2\right)$ und wie was schließt du daraus?

3. BMI von 30,4 dieser Wert liegt im Normbereich

4. BMI von 30,4 der Patient leidet unter Adipositas

5. BMI von 26,8 damit ist der Patient untergewichtig

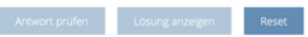

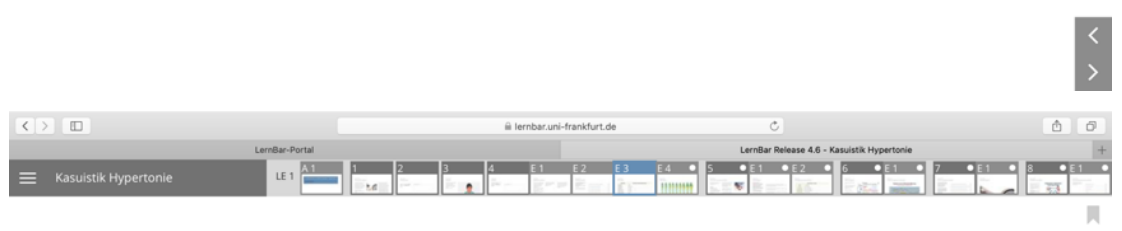

Kasuistik Hypertonie

BMI Einteilung
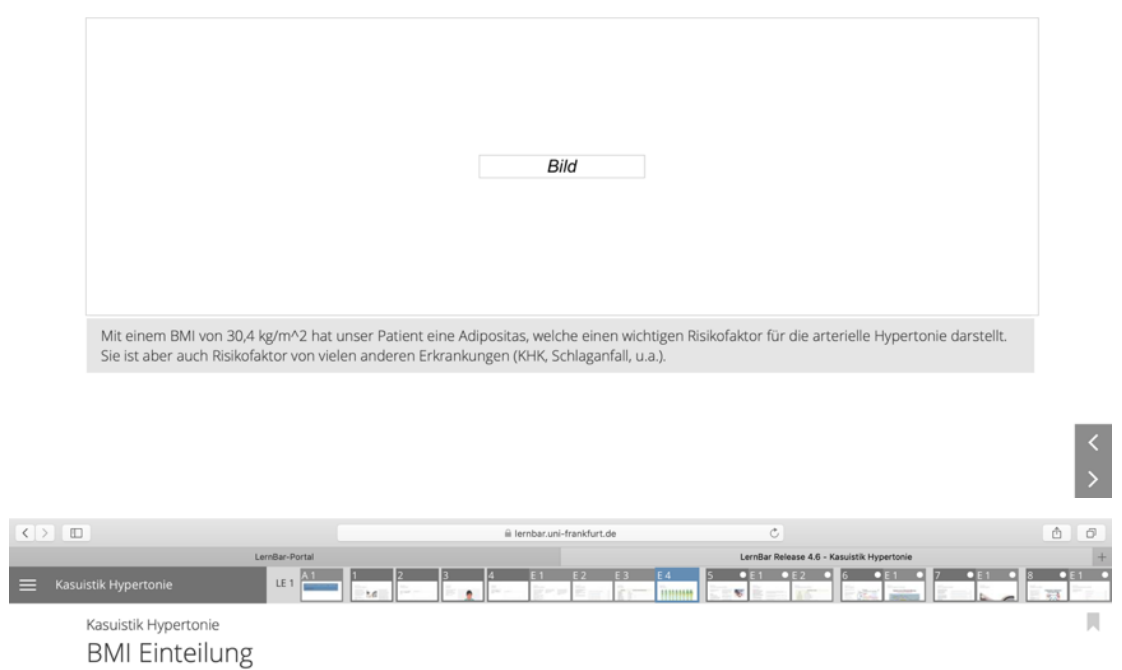

Bild 


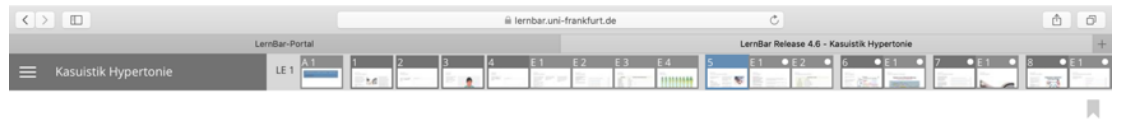

Kasuistik Hypertonie

Kehren wir zurück zu unserem Patienten...

Nach Anamnese und kurzem Exkurs zum BMl erfolgt nun die körperliche

Untersuchung:

pas Herz und die Lunge sind ohne Befund.

Abdomen ist die Leber $2 \mathrm{~cm}$ unter den

Rippenbogen, ansonsten ist auch das Abdomen ohne Befund.

Bild

Inspektion:

Vitalparameter: Der Puls von Herrn J.B. ist

Ernährungszustand und einen

attersentsprechenden Ailgementinzustand

auszumachen.

185/105 mmHg links $185 / 110 \mathrm{~mm}$

Wie du eine korrekte Blutdruckmessung $($ (wnw) anschauen.

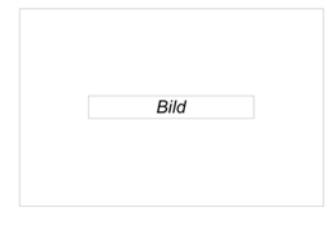

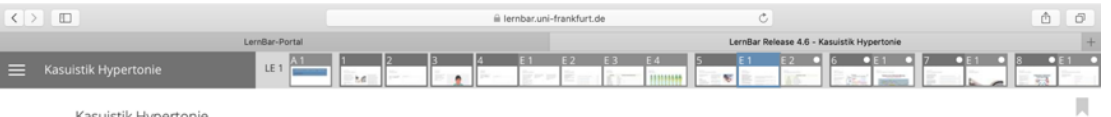

Kasuistik Hyperton

Zusammengefasst...

Zusammenfassend hast du

herausgefunden, dass Herr J.B. diverse

aufweist (Nikotinabusus, Den Hyperton

mellitus, Hypercholisterinämie

dipositas). Die Pràvalenz der Hypertonie

kann mit dem Risikofaktor Adipositas bis

zu $75 \%$ sein.

spricht für eine arterielle Hypertonie.

Wie sicherst du deine Verdachtsdiagnose

"Arterielle Hypertonie?

1. Ich messe eine halbe Stunde nach der ersten Messung noch einmal den Blutdruck, da bei der ersten Messung auch die Aufregung des Patienten den erhohten Druck verursacht haben könnte.

2. Ich brauche keine weitere Absicherung, da an beiden Armen ein systolischer Wert $O$ von $185 \mathrm{mmHg}$ gemessen wurde.

3. Ich schicke den Patienten vorerst nach Hause und bestelle ihn für den nächsten ag zur erneuten RR-Messung ein.

4. Ich ordne eine arterielle RR-Messung an, nur so kann man die Diagnose sichern. $\mathrm{O}$ Hause messen, um auszuschließen, dass die Hypertonie durch Nervosität verursacht wird

Newert priten

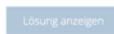

Reser

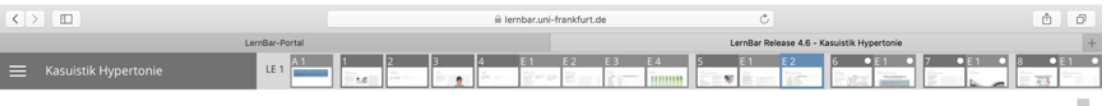

Kasuistik Hyperton

Schweregrade der Arteriellen Hypertonie

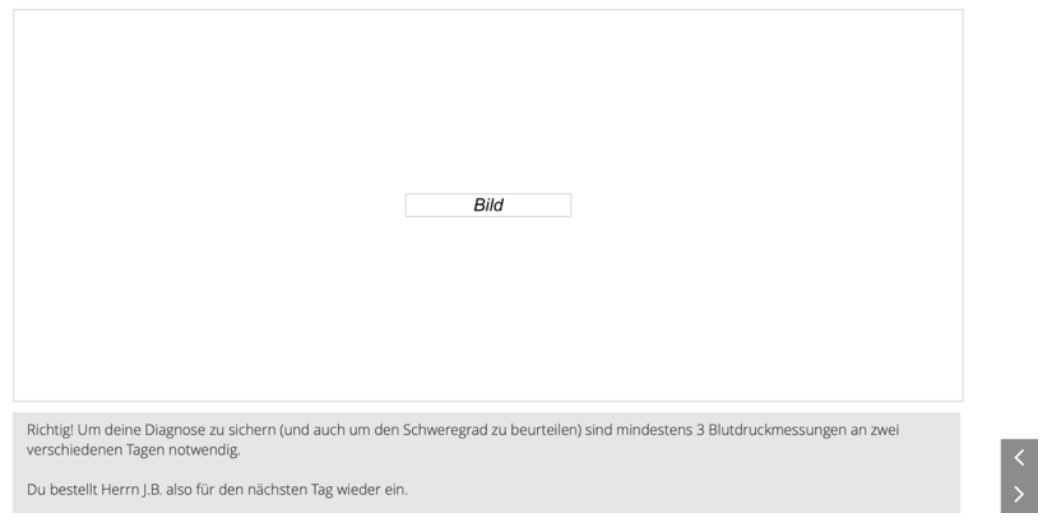




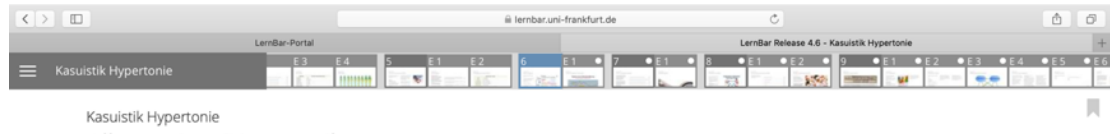

Kasuistik Hypertonie

Allgemeine Diagnostik

Zur Basis-Diagnostik gehören folgende Punkte:

Anamnese und kôrperliche Untersuchung (Schwindel, Ohrensausen, Nervosität,

Präkordialschmerz, Herzklopfen,

Langzeit-Blutdruckmessung

Labor: Kreatinin, Elyte, Lipide, Glukose Harnstoff und TSH.

Im Urin: Glukose und Protein

EKG und Echokardiographie

Abdomen-Sonographie
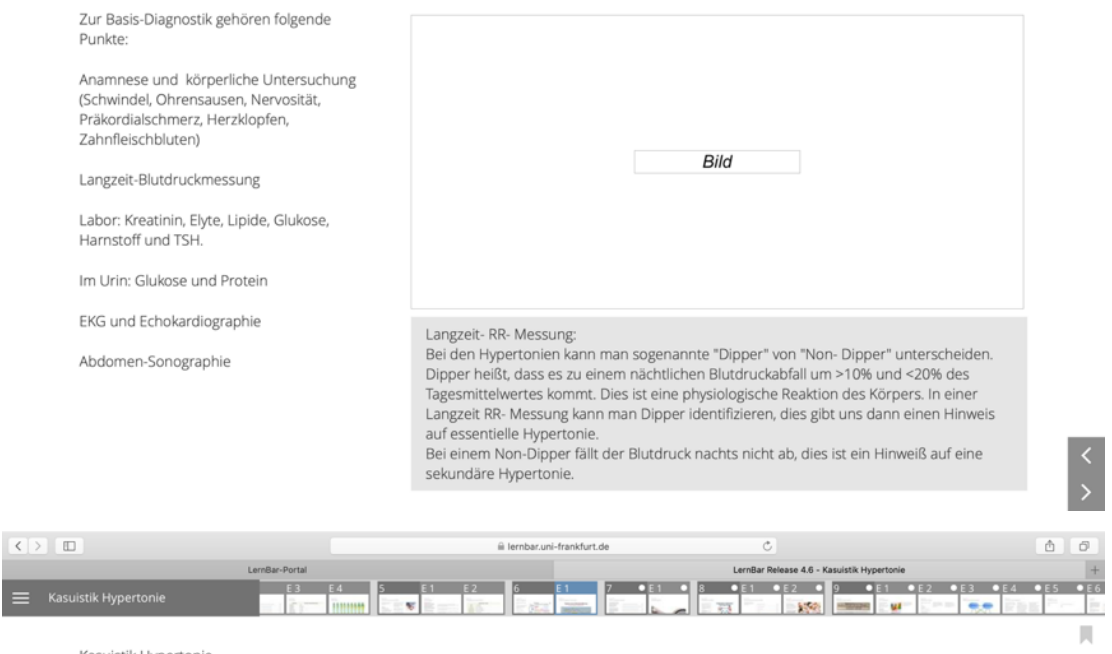

Kasuistik Hypertonie

So sieht eine mögliche 24-h- Messung aus:

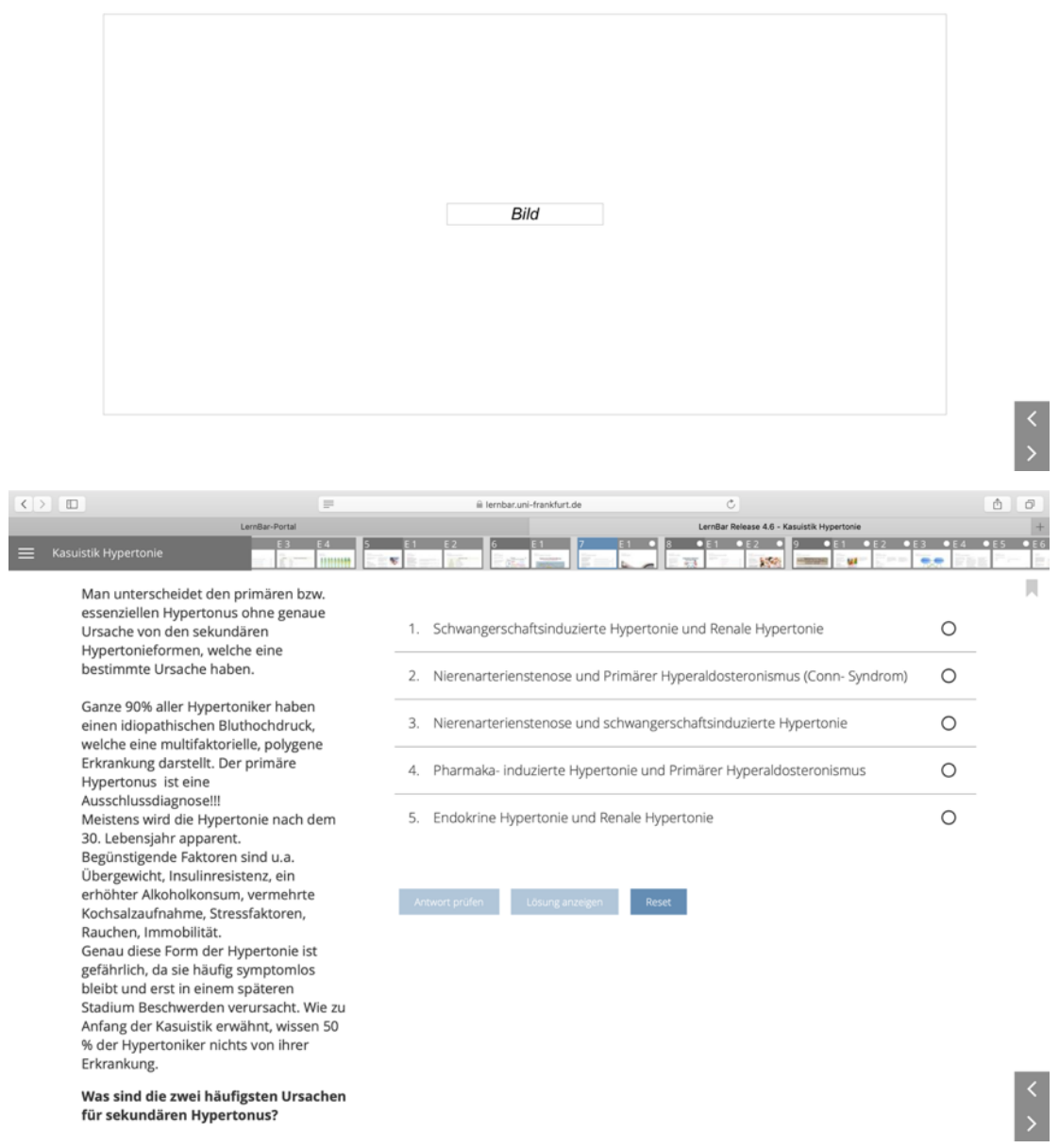



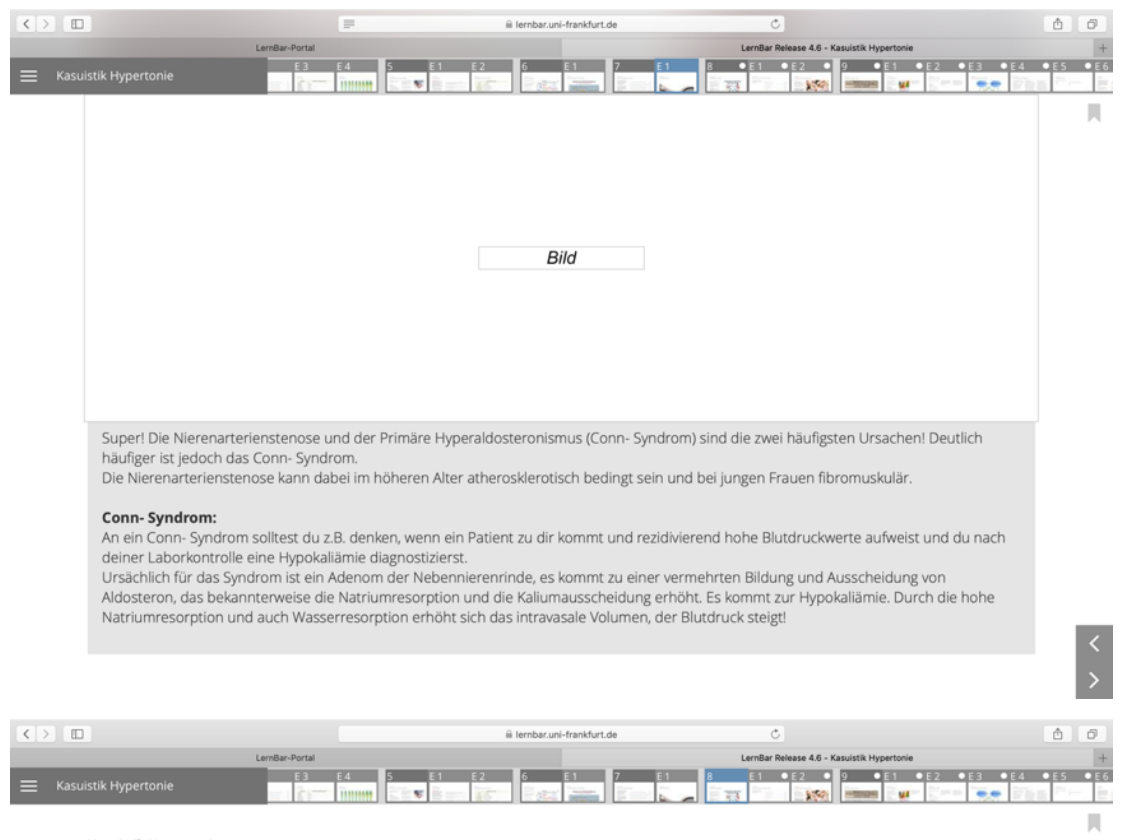

Kasuistik Hypertonie

Komplikationen und Notfälle

Es gibt zwei relevante Notfallsituationen:

Zum einen spricht man von der

Hypertensiven Krise und zum anderen vom

Hypertensiven Notrall. Entscheldendis.

$230 / 130 \mathrm{mmHg}$

Hypertensive Krise: Hypertonie Grad 3

(>180/110mmHg) ohne Symptome eines

Organschadens

Die genauen Blutdruckwerte, ab wann es

sich um eine hypertensive Krise bzw.

Notfall handelt varilieren in der Literatur

zwischen $180-240 \mathrm{mmH}$ ig systolisch und

$110-120 \mathrm{mmH}$ H diastolisch. Die

angegebenen Werte entsprechen der

Leitlinie. Wichtiger ats hypertonie

Zahlenwerte, ist es zu erkennen wann es

sich um einen hypertensiven Notfall

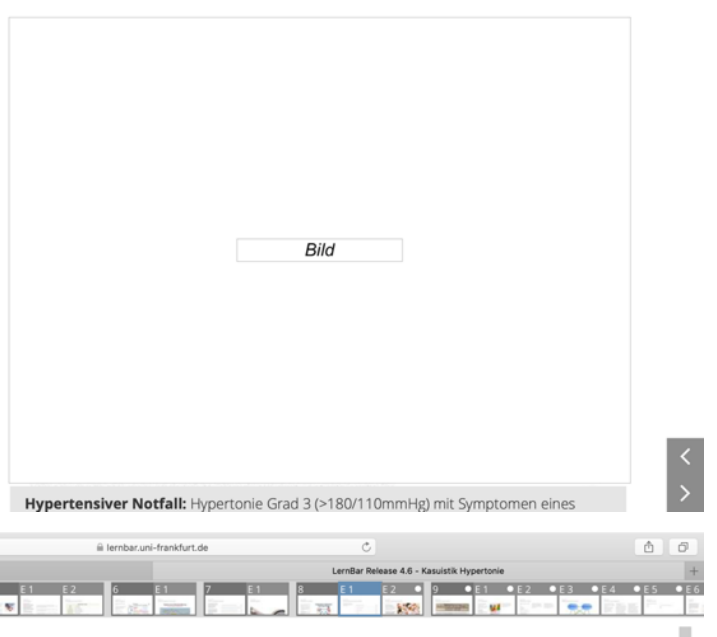

Kasuistik Hypertonie

Was tun bei hypertensiver Krise oder Notfall?

Welche Medikamente sind Mittel der ersten

Notfall?

\begin{tabular}{lc} 
1. Nitroglycerin (Glyceroltrinitrat) & $\square$ \\
\hline 2. ACE-Hemmer wie Captopril & $\square$ \\
\hline 3. Urapidil und Clonidin & $\square$ \\
\hline 4. Diuretika & $\square$ \\
\hline 5. Adrenalin & $\square$
\end{tabular}

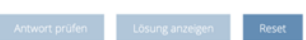



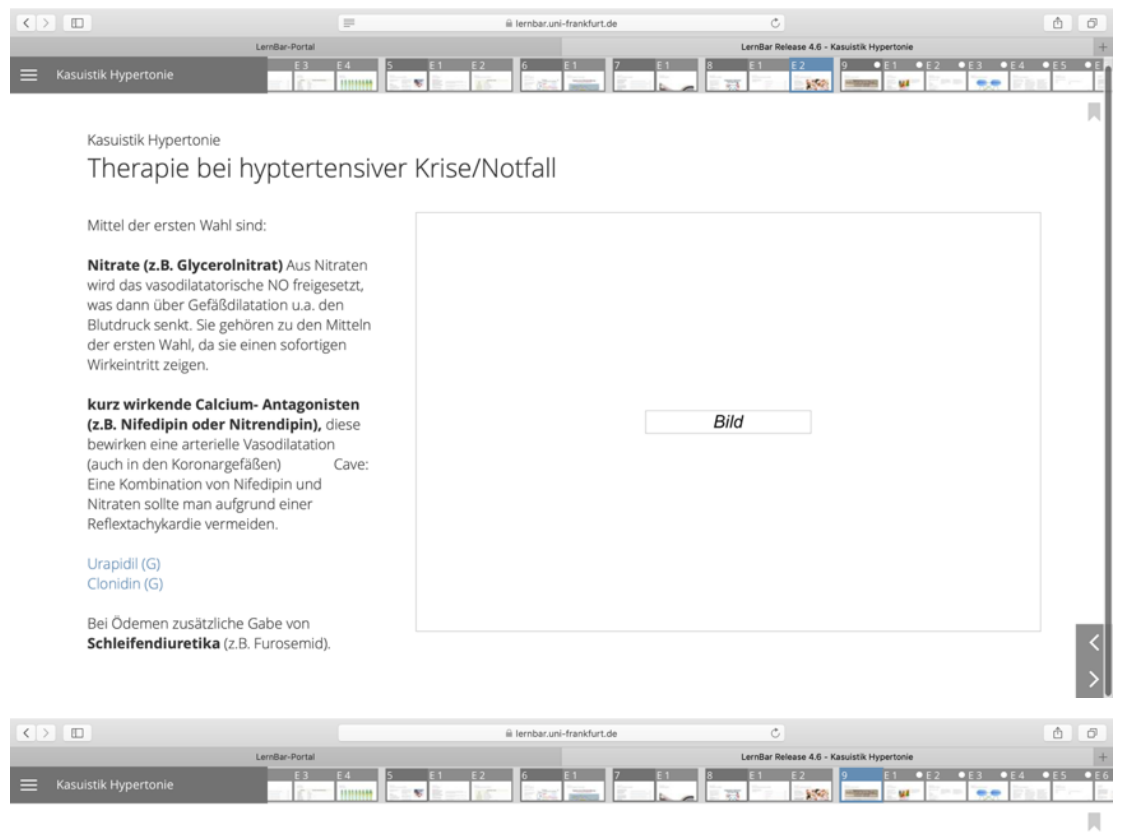

Kasuistik Hypertonie

Therapie der Arteriellen Hypertonie
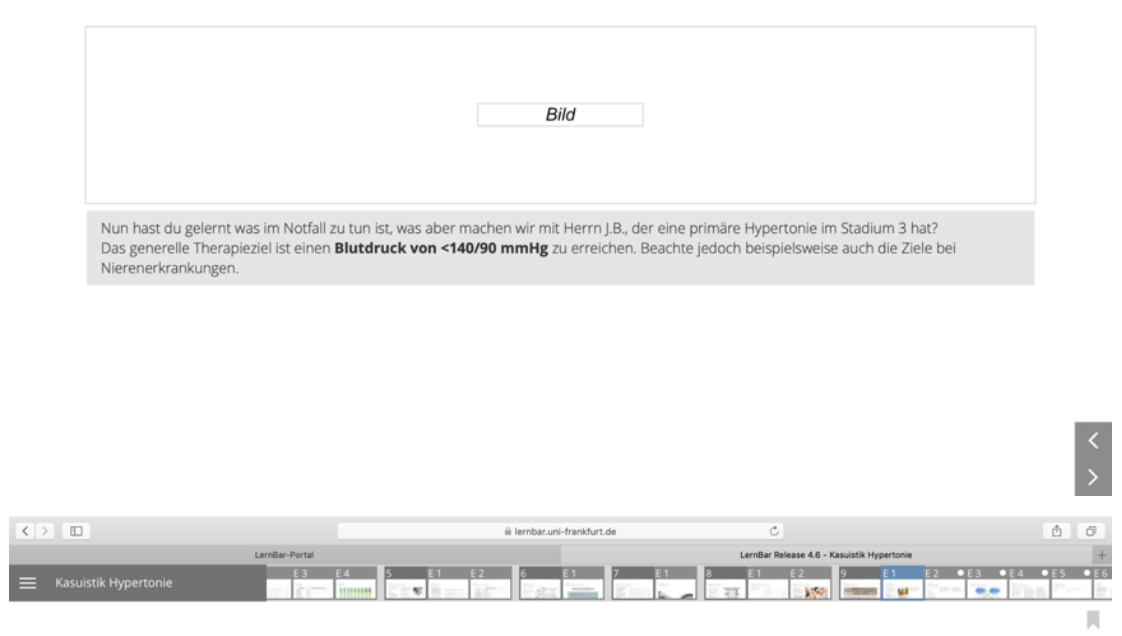

Kasuistik Hypertonie

Erster Therapieschritt

Du weißst aus deiner gründlichen

Anamnese, dass dein Patient einige

Allomeinma Bnatmen ar Änderung des

Lebensstils ist Basis der Hypertonie.

Therapie.

\section{Gewichtsreduktion}

Rauchen einstellen, Kaffeekonsum un C2- Konsum sollten vermindert werden

hypertoniebegünstigende Medikamente weglassen

Sport wie Ausdauertraining (Laufen

Walking, Schwimmen)

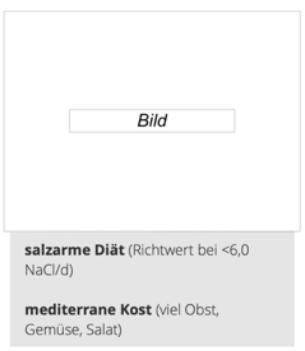

Interessant ist, dass bis zu 25\% der leichten

Hypertonien (Stadium 1) durch

therapient werden kosismen.

andere Risikofaktoren minimieren 


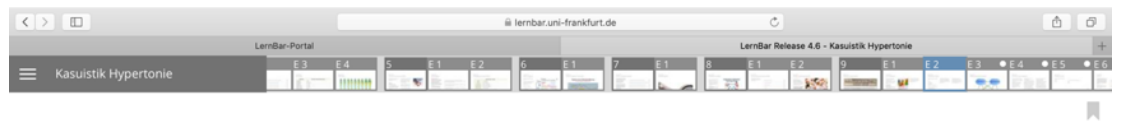

Kasuistik Hypertonie

Medikamentöse Therapie

5 Medikamentengruppen sind Mittel der

Für diese Medikamentengruppen ist ein

Waht

Thiazide
ACE-Hemn worden, die kardiovash

Alle diese Medikamente sind zur

Monotherapie geeignet. Dies ist auch de

Zusätzlich beginnt man mit einer niedrigen

AT1-Rezeptorblocker gesenkt werden.

Dosis, die man ggf. steigern kann.

Beta- Blocker

langwirksame Kalziumanatgonisten

Das kannst du dir mit "ABCD" ganz gut

merken (ACE-Hemmer/AT)

Calciumantagonisten, Diuretika)
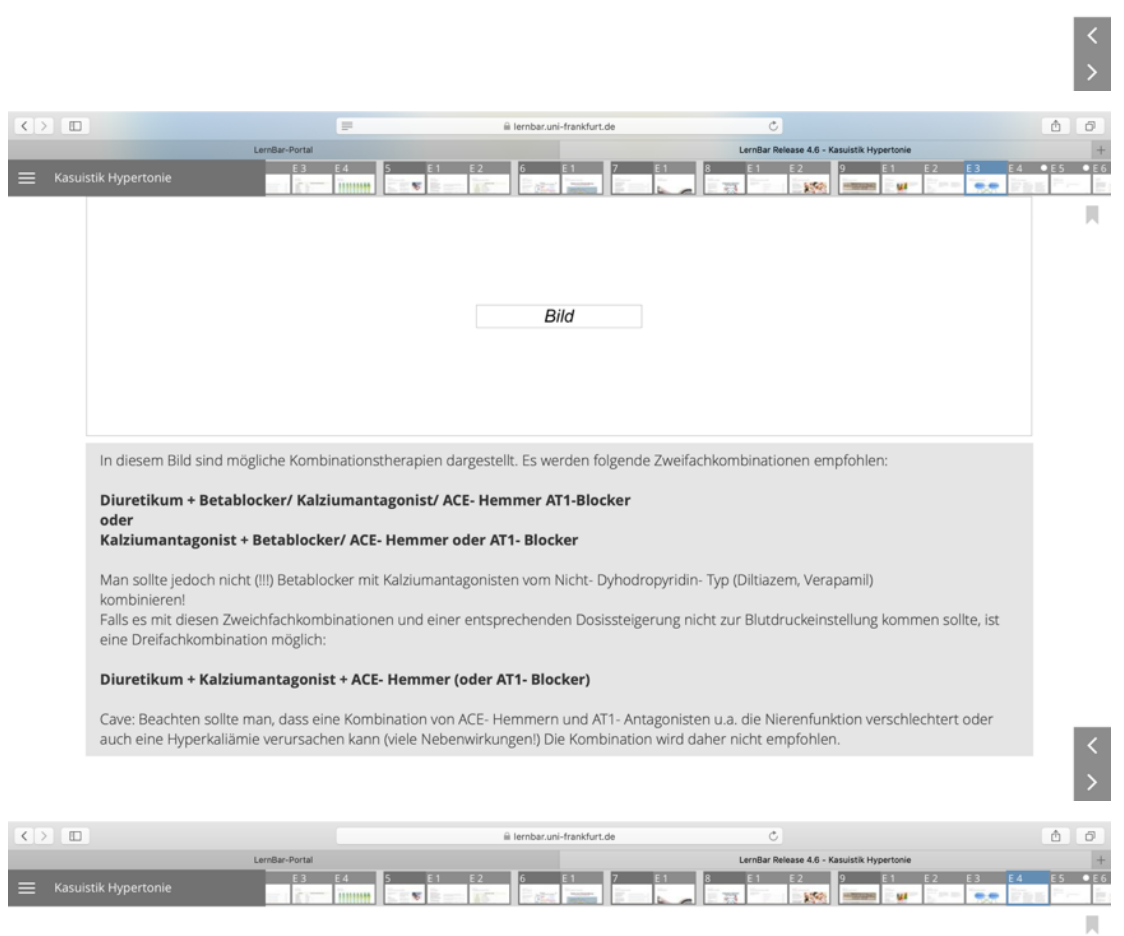

Kasuistik Hypertonie

ACE-Hemmer und AT1-Antagonisten

ACE- Hemmer sind Mittel der Wahl bei Hypertonie, so viet ist nun klar. Jedoch gibt

beachten sollte

Angioodem Aontraindikation getter:

Schwangerschaft und Stillzeit

Relativ kontraindiziert sind

Nierenarterienstenosen, gestörte
Pathophysiologie ACE- Hemmer be

Kurz zusammengefasst kommt es bei der Gewebeumbauz in den Glomeruli zum

, wodurch ein erhöhter Perfusionsdruck benötigt wird um eine ausreichende Filtration zu gewahrleisten ACE-Hemmer jedoch senken den

Pefusionsornck und an dendem cloment mar aber viel Bradykinin).Bei

Niereninsuffizienten kann diese Situation hicht ausgegichen werden, es wird nicht ungenügend filtriert und ein akutes
Die ACE- Hemmer schädigen auch die Niere des Fötus, weshalb die

Kontraindikation darstellt der Niereninsuffizienz ist es bei Fötus: die Niere ist noch nicht entwickelt und benotigt einen höheren Filtrationsdruck! Nun ist auch klar, weshalb ACE- Hemmer (die den Pentusionsaruck senkent) beim Fotus ein 


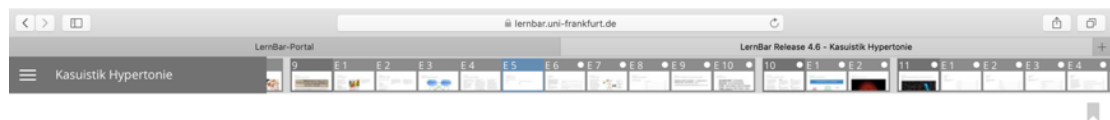

Kasuistik Hypertonie

Therapie von Herrn J.B.

Was für ein Medikament verschreibst du

Herrn!B?

"Torem 10 mg" (Torasemid, Schleifendiuretikum)

$\checkmark$ Tipp

chau noch mal im vorherigen Abschnit.

$\square$

$\square$

$\square$

4. Metoprolol $50 \mathrm{mg}$

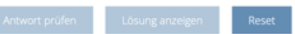

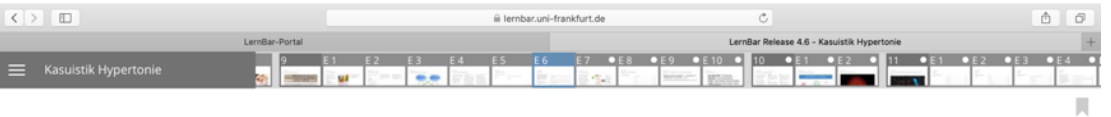

Kasuistik Hypertonie

Und nun?..

Ganz genau. Als erstes beginnen wir mit

einer niedrigen Dosierung und mit dem

$2.5 \mathrm{mg}$ ist hier eine gute Wahl, solange keine ACE- Hemmer- Unverträglichkeit bekannt ist.

1. Ich verschreibe ihm zunächst einen Hustensaft und bestelle ihn für nächste Woche wieder ein.

O

2. Ich wechsie die Medikation vom ACE-Hemmer auf einen AT1-Antagonisten. $O$

Nachdem du Herrn J.B. zur

Wiedervorstellung in 2 Wochen wieder

Reizhusten. Was nun?

3. ICh erniedrige die Dosierung des ACE- Hemmers und bestelle den Patienten zu
Kontrolle für nächste Woche ein.

4. Dies ist kein Grund zur Sorge, ich empfehle dem Patienten viel Tee zu trinken.

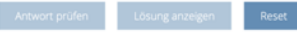

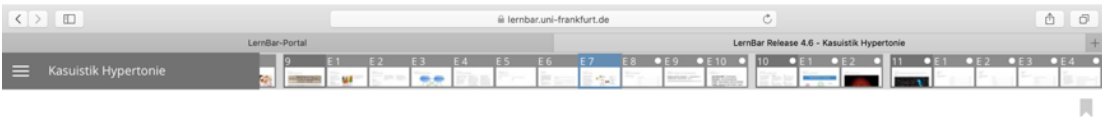

Kasuistik Hypertonie

"ACE-Hemmer-Husten"

Dies ist genau die richtige Antwort. Der

sogenannte ACE-Hemmer- Husten ist en

Reizhusten, dervermuttich durch

Angiotensin- Converting- Enzyme hat

neben der Spaltung des Angiotensin I zu

Angiotensin II auch die Aufgabe Bradykinin

abzubauen. Durch den ACE-Hemmer ist

vermentr Bradyknininvorianden.

Wer Reizhusten soitte dir immer ein

Warnsignal seh, daher solttest du

ACE-Hemmer Medikation gezielt danach

fragen. Herr J.B. hat also nun diese

Unverträglichkeit, du wechselst damit seine

Medikation auf einen AT1- Antagonisten

(z.b. Valsartarn

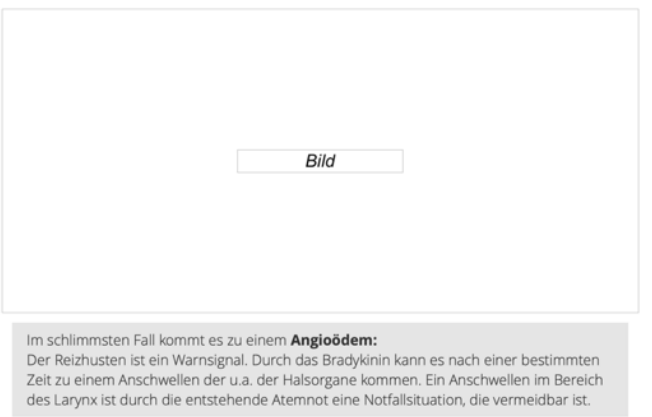

des Larynx ist durch die entstehende Atemnot eine Notfallsituation, die vermeidbar ist. 


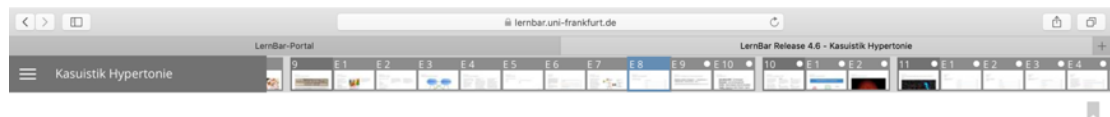

Kasuistik Hypertonie

Hypertonie in der Schwangerschaft

Was ist das Mittel der 1 . Wahl bei

Hypertonie in der Schwangerschaft?

\begin{tabular}{lc} 
1. Selektive Beta- Blocker & 0 \\
\hline 2. Nifedipin & 0 \\
\hline 3. Ramipril & 0 \\
\hline 4. Valsartan & 0 \\
\hline 5. Alpha- Methyldopa & 0
\end{tabular}

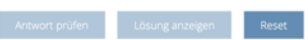

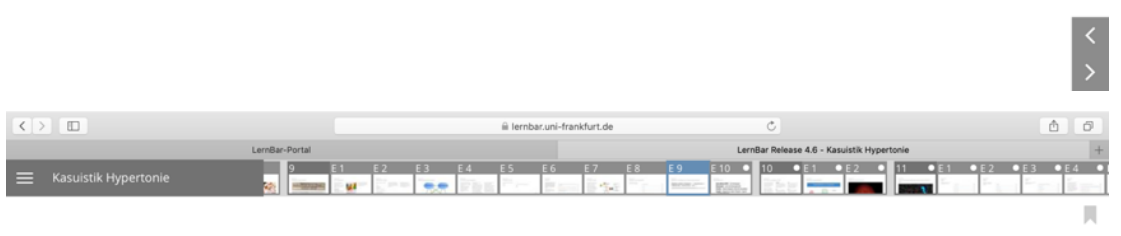

Kasuistik Hypertonie

Hypertonie in der Schwangerschaft - Wirkung von Alpha-Methyldopa

Bild

Alpha- Methyldopa, ein Sympathotonikum ist Mittel der 1. Wahl bei einer Schwangerschaftshypertonie.

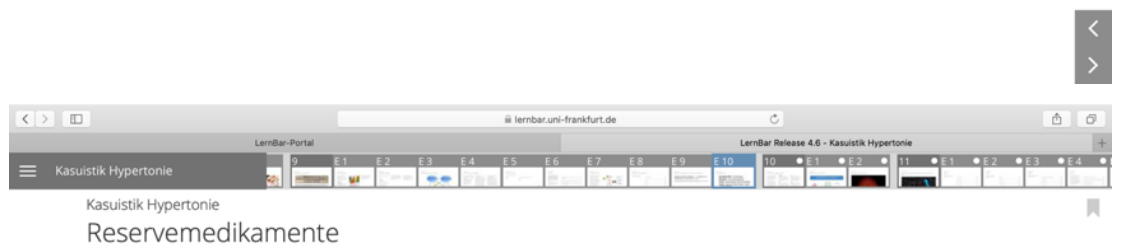

Reservemedikamente

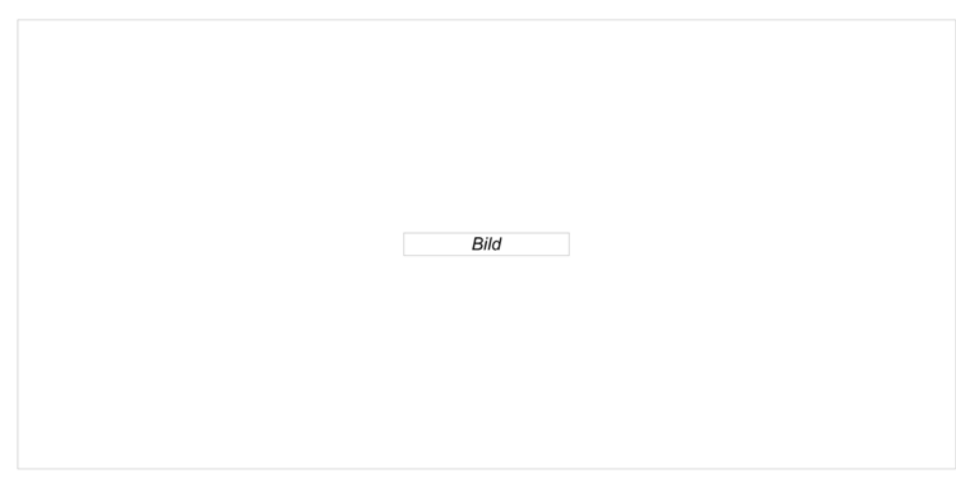

Hier noch Reservemedikamente für die Hypertonietherapie. V.a. Alpha- Methyldopa und Clonidin solltest du kennen. 


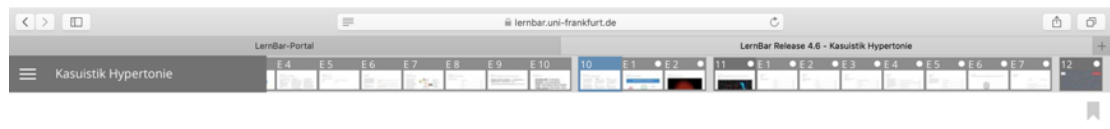

Kasuistik Hypertonie

Wir kommen nun so langsam zum Ende...

So, du hast bis jetzt schon einiges gelernt

von Átiloogie uber Diagnostik bis zur Komplikationen und Folgen der Hypertonie Bescheid weilit.

Der Bluthochdruck schädigt das

Gefabsystem, v.a. in kieinen Gefaben

kommt es zu veránderungen des

Organ zeshädigungen kommen. Wir

beschränken uns auf die häufigsten und

wichtigsten
Gefäßsystem

Wichertoniker entwickeln eine $2480 \%$ einen Hypertonus zur Ursache. Komplikation darstellen.

Herz:

Durch Makro- und Milkoangiopathie

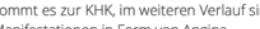

ectoris, Herzinfarkt, Linksherzinsuffit

Herzrhychmusstorrungen oder der

plötzliche Herztod möglich.

Bei der hypertensiven Kardiornyopathie

kommt es zur Druckbelastung des Herzens,

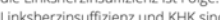

Todesursache be i 23 ader sind
Auge

Der Hypertonus verändert die Gefä̉e der

Retina, es ko

hypertonicus/Hypertensive Retinopathie. Auch hier kann es arteriosklerotische

wandverdickungen geben.

Niere:

Bei der hypertensiven Nephropathie

terminal entwickelt sich eine

Schrumpfniere und der Patient befindet

sich im Nierenversager.

Gehirn:
Die zerebrale Ischămie und der Schlaganfala

ind geturchtete Komplikationen. Aber

(sehr viel seltener) sind möglich.

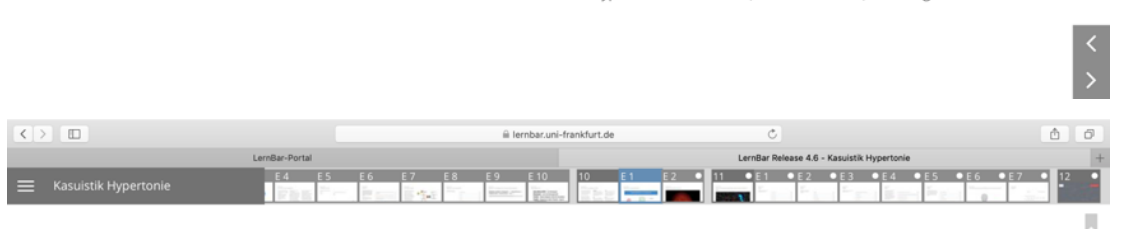

Kasuistik Hypertonie

Wiederholung - Hauptkomplikationen

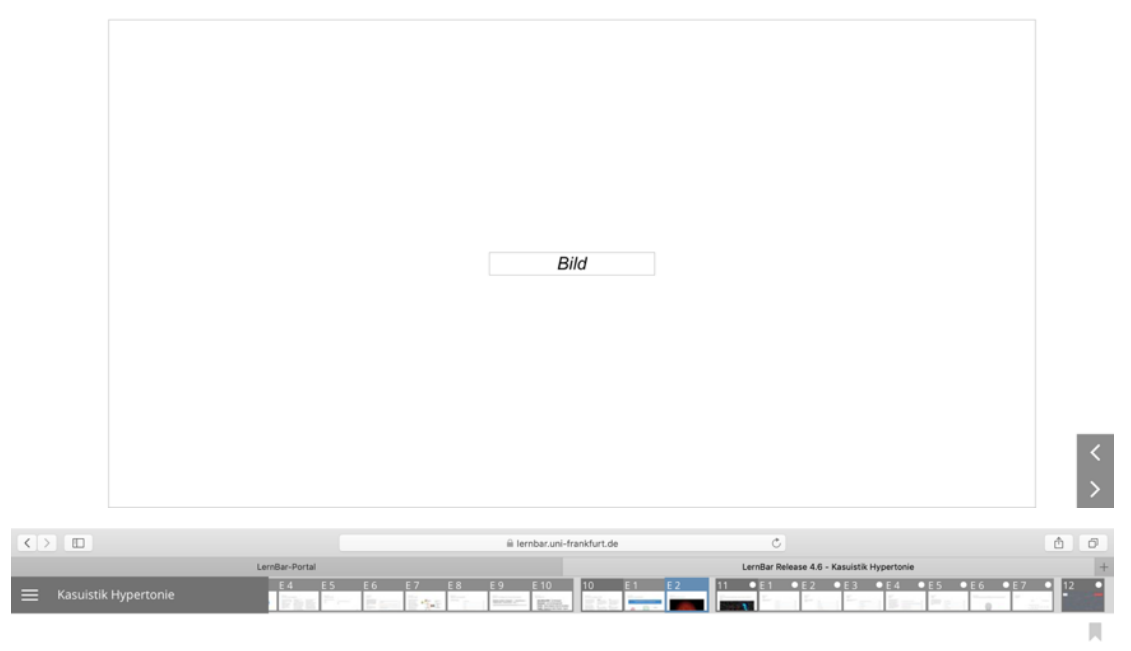

Kasuistik Hypertonie

Hypertensive Retinopathie Stadium IV 


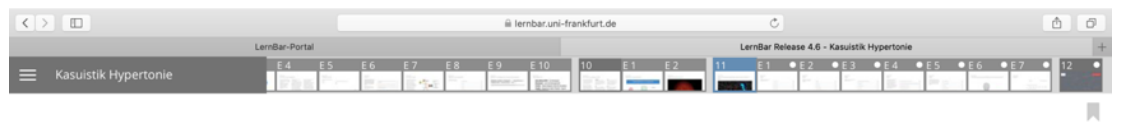

Kasuistik Hypertonie

Super, nun ist die Kasuistik abgeschlossen. Zum Schluss noch 5 Fragen zum üben :)

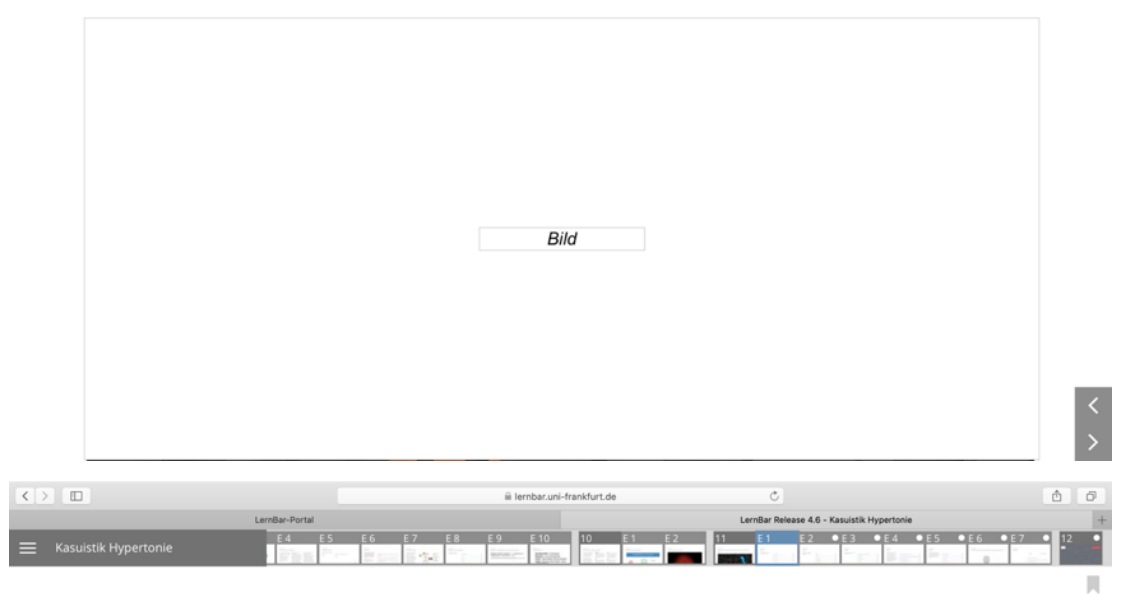

Kasuistik Hypertonie

Frage 1

Bei welchem Blutdruck spricht man vor

\begin{tabular}{ll} 
1. $200 / 100 \mathrm{mmHg}$ & $\mathrm{O}$ \\
\hline 2. $210 / 110 \mathrm{mmHg}$ & $\mathrm{O}$ \\
\hline 3. $220 / 120 \mathrm{mmHg}$ & $\mathrm{O}$ \\
\hline 4. $230 / 130 \mathrm{mmHg}$ & $\mathrm{O}$ \\
\hline 5. $240 / 140 \mathrm{mmHg}$ & $\mathrm{O}$
\end{tabular}

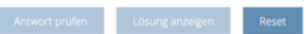

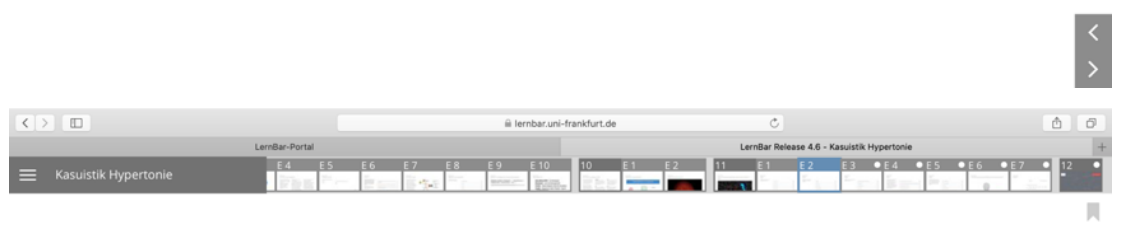

Kasuistik Hypertonie

Frage 2

Was gehört NICHT zu den typischen

Hypertonie?

\begin{tabular}{lc} 
1. Adipositas & 0 \\
\hline 2. Nikotinabusus & 0 \\
\hline 3. Diabetes mellitus & 0 \\
\hline 4. Hypercholesterinämie & 0 \\
\hline 5. erhöhter Vitamin-D-Spiegel & 0
\end{tabular}

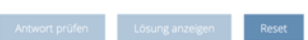




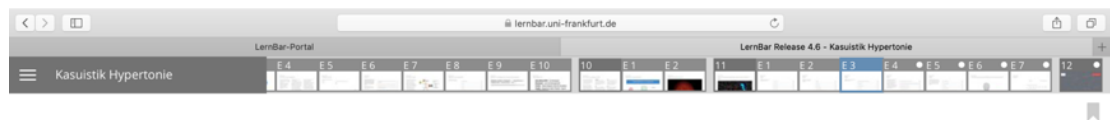

Kasuistik Hypertonie

Frage 3

Was gehört NICHT zu den Ursachen eines

sekundären Hypertonus?

\begin{tabular}{lc} 
1. pAVK (periphere arterielle Verschlusskrankheit) & 0 \\
\hline 2. Schwangerschaft & 0 \\
\hline 3. Nierenarterienstenose & 0 \\
\hline 4. Conn- Syndrom & 0 \\
\hline 5. endokrine Hypertonie & 0
\end{tabular}

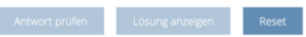

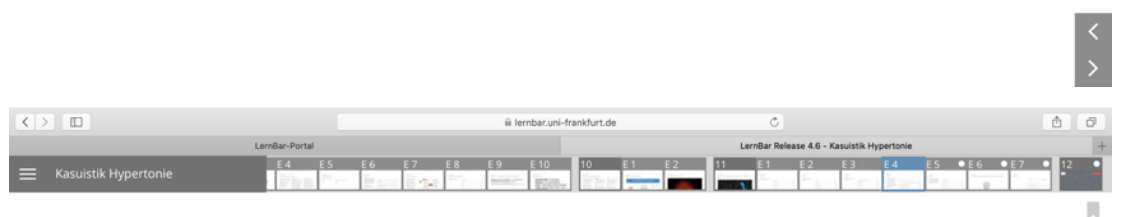

Kasuistik Hypertonie

Frage 4

Eine 62-jăhrige Patientin mit essenzieller

arterieller Hypertonie hat unter

Behandlang min Hydrochlorothiazid eine

gezeigt. Es wird jerzz eine leichte

Linksherzinsuffizienz festgestellt. Welche

der folgenden Maß̉nahmen ist als nächste

- unter Beachtung der Richtinien fur ene

vorsichtige Therapieumstellung - am

\begin{tabular}{ll}
\hline 1. Ersatz des Hydrochlorothiazids durch Dihydralazin & O \\
\hline 2. Ersatz des Hydrochlorothiazids durch ein Reserpinhaltiges Antihypertensivum & 0 \\
\hline 3. zusätzliche Behandlung mit Doxazosin & 0 \\
\hline 4. zusătzliche Behandlung mit Enalapril & 0 \\
\hline 5. zusătzliche Behandlung mit Furosemid & 0
\end{tabular}

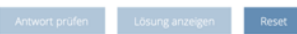

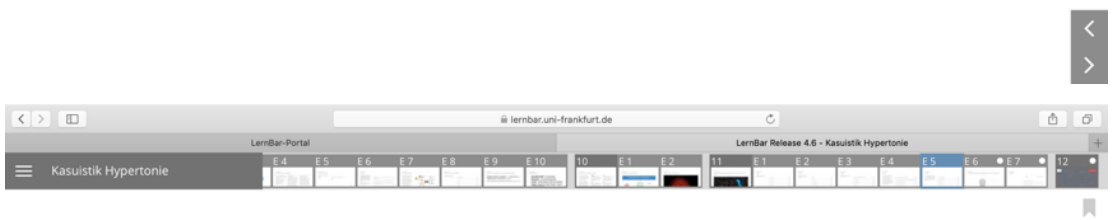

Kasuistik Hypertonie

Frage 5

Bei einem 55-jährigen Patienten wird die

Diagnose einer sekundaren Hypertonie be

ats

Nierenarterieneinengung vorrangig in

\begin{tabular}{ll} 
1. fibromuskuläre Dysplasie & 0 \\
\hline 2. Nierenarterienaneurysma & 0 \\
\hline 3. Arteriosklerose & 0 \\
\hline 4. Nierenarterienhypoplasie & 0 \\
\hline 5. Kompression durch benachbarten raumfordernden Prozess & 0
\end{tabular}

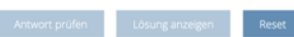




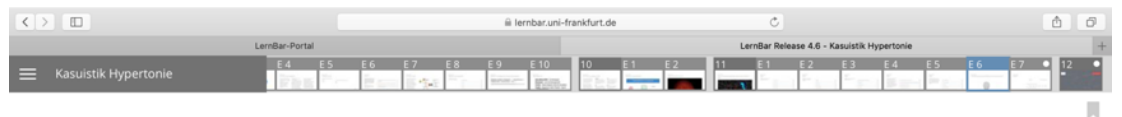

kasulsuk Hyperton

Super! Du hast die Kasuistik geschafft! Bitte evaluiere noch kurz die Kasuistik!

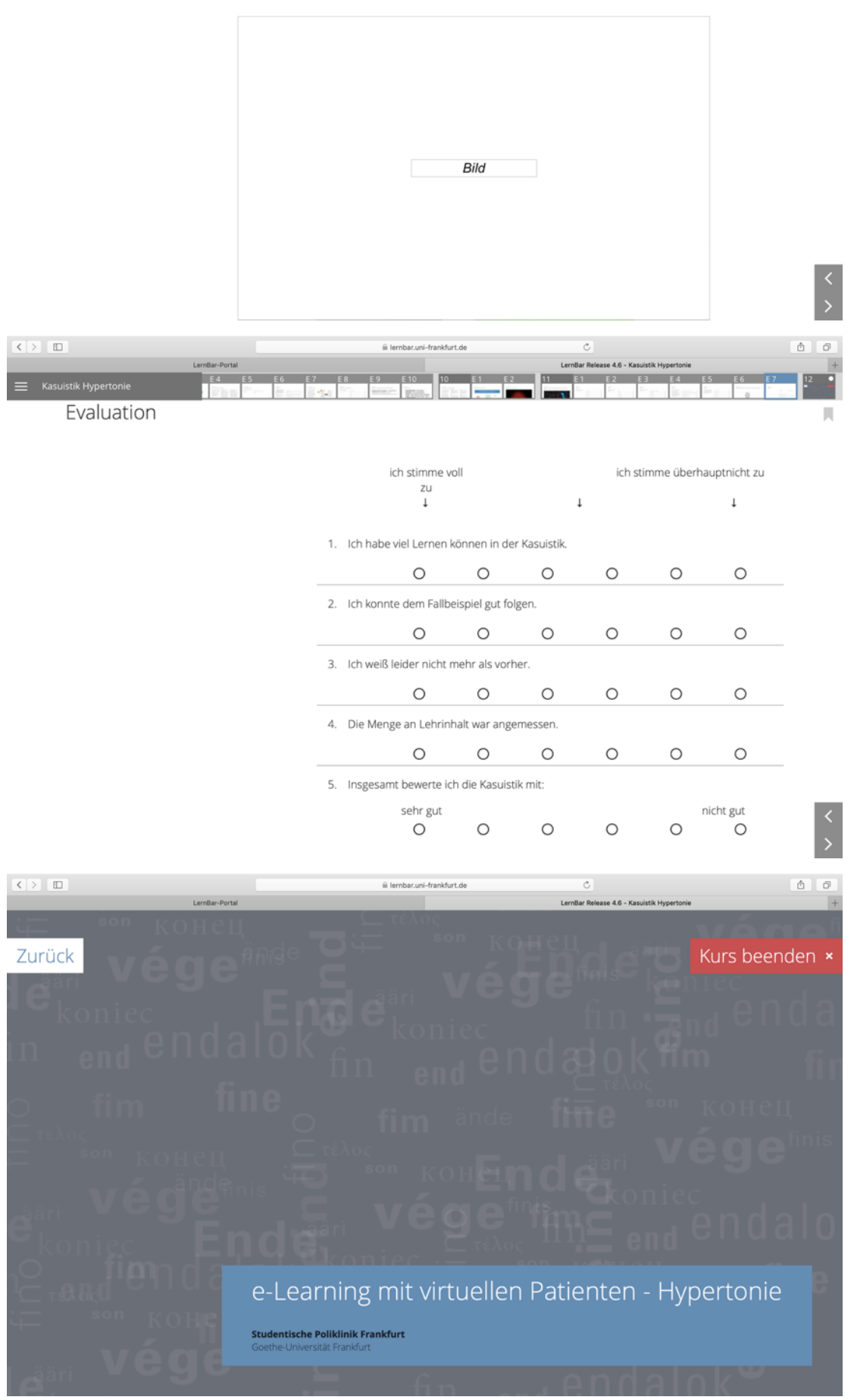




\subsection{Theoretischer Langzeit-Test}

1. Welches der folgenden Medikamente wird nicht zur Eradikationstherapie von H. Pylori Infektion der Magenschleimhaut eingesetzt?
A) Amoxicillin
B) Clarythromycin
C) Metronidazol
D) Ciprofloxacin
E) Bismuth

2. Ihr Pat., Rudi Rakete, klagt seit Wochen über epigastrische Schmerzen, die sich nach dem Essen bessern, sowie einen sehr schwarzen Stuhl. Durchfälle und Flatulenzen werden verneint. Die körperliche Untersuchung ist bis auf einen epigastrischen Druckschmerz unauffällig. Die Temperatur des Pat. beträgt $37,2^{\circ} \mathrm{C}$. Ein von Ihnen durchgeführter Guajak-Test fällt positiv aus, das Blutbild des Patienten weist auf eine Eisenmangel-Anämie hin. Welche weitere diagnostische Maßnahme ist am wenigsten zielführend?
A) H. Pylori Nachweis mittels 13C-Atemtest
B) Gastroduodenoskopie
C) H. Pylori Nachweis mittels Blutkultur
D) Abdominelle Sonographie
E) H. Pylori Nachweis mittels Urease Schnelltest

3. Wo ist die bakterielle Gastritis am häufigsten lokalisiert?
A) Fundus
B) Corpus
C) Antrum
D) Pylorus
E) Die bakterielle Gastritis ist in allen Teilen des Magens gleich häufig zu finden.

4. Sie diagnostizieren bei Ihrem Patienten (50 J., männlich, BMI: $30,4 \mathrm{~kg} / \mathrm{m}^{2}$, Raucher) eine primäre Hypertonie. Welche der folgenden Maßnahmen ist nach der Erstdiagnose nicht indiziert?
A) Gewichtsreduktion
B) Gabe von Alpha-Methyl-DOPA
C) Gabe eines ACE-Hemmers
D) Rauchen einstellen
E) Sportliche Betätigung

5. Welches der folgenden Krankheitsbilder ist ein Grund für einen sekundären Hypertonus?
A) Hypovolämie
B) Hypothyreose
C) Karotissinussyndrom
D) Morbus Addison
E) Nierenarterienstenose

6. Ab welchem Blutdruck- Wert spricht man von einer Hypertonie (Stadium 1)?
A) $>120 / 80 \mathrm{mmHg}$
B) $>80 / 60 \mathrm{mmHg}$
C) $>140 / 90 \mathrm{mmHg}$
D) $>170 / 105 \mathrm{mmHg}$
E) $>180 / 110 \mathrm{mmHg}$ 
7. Welche Antibiotika gehören üblicherweise zur Standardtherapie der (typischen, nichtmultiresistenten) Tuberkulose?
A) Ethambutol + Linezolid
B) Isoniazid + Rifampicin
C) Doxycyclin + Ethambutol
D) Pyrazinamid + Levofloxacin
E) Isoniazid + Clindamycin

8. Welche Aussage zur Diagnostik und Therapie der Tuberkulose trifft zu?

A) Der Quantiferontest erlaubt die Unterscheidung zwischen latenter Infektion und aktiver Erkrankung.

B) Bei der Tbc-Erstinfektion kommt es fast immer zu grippeähnlichen Symptomen.

C) Patienten mit offener Tuberkulose müssen in den meisten Fällen nicht isoliert werden.

D) Eine im Ausland erworbene Tuberkulose ist nicht meldepflichtig.

E) Unter einer immunsuppressiven Therapie kann es zur postprimären Tuberkulose kommen.

9. Welche der genannten Maßnahmen kommt am wenigsten in Betracht, wenn auf einer Krankenhausstation bei einem Patienten eine offene Lungentuberkulose diagnostiziert wird?
A) Isolierung des Patienten durch Unterbringung im Einzelzimmer
B) Meldung an das zuständige Gesundheitsamt
C) spezielle Entsorgung der mit infektiösem Material kontaminierten Abfälle
D) umgehende BCG-Impfung bei den Tuberkulin- positiven Kontaktpersonen
E) Röntgenuntersuchung des Thorax bei Kontaktpersonen, bei denen im aktuellen zeitlichen Zusammenhang eine Tuberkulinkonversion stattgefunden hat.

10. Welche der folgenden Gründe ist kein positiver Faktor für eine Chronifizierung von Kreuzschmerzen (sogenannte "yellow-flag")?
A) Körperliche Schwerarbeit in monotoner Körperhaltung
B) Fieber
C) Depressivität
D) Verlust des Arbeitsplatzes
E) Private oder Berufliche Unzufriedenheit

11. Herr K.M. kommt in Ihre Praxis und berichtet über Schmerzen, die am Vortag beim Heben einer Kiste plötzlich im Bereich der rechten LWS aufgetreten sind. Nach einer genauen Anamnese erfahren Sie, dass die Schmerzen bewegungsabhängig sind und in den Oberschenkel ausstrahlen. Die Ausstrahlung endet oberhalb des Knies und der Patient gibt keinerlei Taubheitsgefühl an. In der Körperlichen Untersuchung stellen Sie eine schmerzbedingte Bewegungseinschränkung der LWS fest, keine Sensibilitätsstörungen oder Kraftminderungen feststellbar. Herr M. ist ansonsten in gutem Allgemeinzustand. Bei den beschriebenen Symptomen handelt sich mit hoher Wahrscheinlichkeit um welche Krankheit?
A) Extraradikuläre Kreuzschmerzen
B) Radikuläre Kreuzschmerzen
C) Komplizierte Kreuzschmerzen
D) Unkomplizierte Kreuzschmerzen
E) Extravertebrale Kreuzschmerzen

12. Das Ott-Maß (z.B. Ott $30 / 34 \mathrm{~cm}$ ) dient am ehesten zur Beurteilung der...
A) Beugefähigheit der Brustwirbelsäule
B) Streckfähigkeit der Brustwirbelsäule
C) Beugefähigkeit der Lendenwirbelsäule
D) Streckfähigkeit der Halswirbelsäule
E) Streckfähigkeit der Lendenwirbelsäule 
13. Sie erhalten folgenden Laborbefund: Welche Anämie-Form liegt hier vor und was ist die wahrscheinlichste Ursache dafür? Erythrozyten 4,1 T/ml; Hämoglobin $9 \mathrm{~g} / \mathrm{dl}$; Hämatokrit $30 \%$; MCV 73 fl; MCH 20 pg; MCHC 27,3 g/dl
A) Makrozytäre, hypochrome Anämie - Vitamin B12 Mangel
B) Mikrozytäre, hyperchrome Anämie - Vitamin B12 Mangel
C) Mikrozytäre, hypochrome Anämie - Eisenmangel
D) Makrozytäre, hyperchrome Anämie - Eisenmangel
E) Normozytäre, normochrome Anämie - Vitamin B12 Mangel

14. Was ist die häufigste Ursache für eine mikrozytäre hypochrome Anämie?
A) Folsäuremangel
B) Hämoglobinmangel
C) Vitamin- B12- Mangel
D) Calciummangel
E) Eisenmangel

15. Womit ist die Anämie im Rahmen eines Intrinsic-Factor-Mangels häufig assoziiert?
A) Bronchialkarzinom (Paraneoplasien)
B) Hämolytische Anämie
C) HIV
D) Typ- A- Gastritis
E) Morbus Crohn

16. Bei einer normalen Gravidität gehört zu den Veränderungen im mütterlichen Organismus am wenigsten:
A) Hyperventilation
B) Erhöhung des Herzminutenvolumens
C) Vermehrung der Blutlipide
D) Diarrhö
E) Ausbildung von Varizen im Bereich von Vulva und Vagina

17. Wie lange dauert eine reguläre Schwangerschaft?
A) 36 Wochen post menstruationem
B) 38 Wochen post menstruationem
C) 40 Wochen post menstruationem
D) 40 Wochen post conceptionem
E) 42 Wochen post menstruationem

18. Die häufigste Ursache einer Schwangerschaftsanämie mit Hämoglobinwerten unter $110 \mathrm{~g} / \mathrm{L}$ ist:
A) ein Eisenmangel
B) ein latenter Folsäuremangel
C) ein Vitamin- B12- Mangel
D) ein erhöhter Folsäurebedarf des Fetus
E) eine verminderte Eisenresorption im Darm

19. und 20. Bei Ihnen wird die 17-jährige Frau Becker vorstellig und klagt über einseitige Kopfschmerzen, die nun schon seit 6 Stunden anhielten. In dieser Zeit hätte sich Frau zweimal übergeben müssen. Außerdem berichtet sie, dass sie vor dem Beginn der Schmerzen "so ein Flimmern im Auge hatte". Auf Nachfrage gibt sie an, dass der Schmerz durch körperliche Anstrengung schlimmer werde. Bei der körperlichen Untersuchung fällt eine deutliche Photophobie auf. Die Temperatur der Pat. beträgt $36,8^{\circ} \mathrm{C}$. 
19. Wie lautet die wahrscheinlichste Verdachtsdiagnose...
A) Meningitis
B) Migräne mit Aura
C) Migräne ohne Aura
D) Spannungskopfschmerz
E) Subarachnoidalblutung

20. ...und welche therapeutischen Sofortmaßnahmen kommen in Frage?

A) Kalkulierte i.v. Antibiose mit Cefotaxim und Ampicillin, zusätzlich Dexamethason i.v.

B) Eisbeutelbehandlung und Auftragen von Pfefferminzöl, sowie Entspannungstherapie

C) Sofortige Überweisung der Patientin in die Stroke Unit via Rettungswagen

D) Behandlung mit ASS 1000 mg i.v., zusätzlich MCP 20 mg i.v., sowie Sumatriptan i.n.

E) Migräneprophylaxe mit Propanolol 80 mg p.o., sowie Amitryptylin 100 mg p.o.

21. Ein 30-jähriger Mann leidet an rezidivierenden Attacken heftiger Kopfschmerzen, die unter Analgetikatherapie nach 1-2 Stunden wieder nachlassen. Welches der genannten Symptome gehört nicht zur Verdachtsdiagnose "Clusterkopfschmerz"?
A) Miosis
B) starkes Schwitzen im Bereich der Stirn und des Gesichts
C) Ptosis
D) Rhinorrhoe
E) Photopsie

22. Der Diabetes mellitus Typ 1 unterscheidet sich vom Diabetes mellitus Typ 2 vorrangig durch:

A) das Entstehen des glykosylierten Blutproteins HbA1c

B) eine erhöhte Nierenschwelle für Glukose

C) eine postprandiale C- Peptid- Konzentration im Blut, die trotz erhöhten Blutzuckerspiegels extrem erniedrigt ist

D) eine Verlangsamung der Nervenleitgeschwindigkeit

E) pathologische Blutzuckerwerte im oralen Glukosetoleranztest

23. Bei einem 57-jährigen, beschwerdefreien Bäckermeister wird anlässlich einer Routineuntersuchung ein Nüchternblutzuckerwert von $137 \mathrm{mg} / \mathrm{dL}$ gemessen. Im daraufhin veranlassten oralen Glukosetoleranztest zeigt sich ein 2-Stundenwert der Plasmaglukose von $266 \mathrm{mg} / \mathrm{dL}$. Es werden außerdem folgende Befunde erhoben: HbA1c $63 \mathrm{mmol} / \mathrm{mol}(7,9 \%)$, Serumkreatinin $0,9 \mathrm{mg} / \mathrm{dL}$, Body-Maß-Index $33,4 \mathrm{~kg} / \mathrm{m}^{2}$. Welche der folgenden Vorgehensweisen ist bei dem Patienten als Erstbehandlung am sinnvollsten?
A) Beginn einer Monotherapie mit Basalinsulin
B) stationäre Aufnahme in eine Diabetesfachklinik zur Gewichtsreduktion
C) Diabetesschulung, Lebensstiländerung und Verordnung von Metformin
D) orale antidiabetische 3-fach- Kombinationstherapie
E) Beginn einer intensivierten Insulintherapie

24. Bei Frau A. wurde ein HbA1c-Wert von $12 \%$ gemessen. Welche der folgenden Aussagen zu diesem Parameter trifft am ehesten zu?

A) Bei Anpassung der Insulindosis anhand des $\mathrm{HbA1c}$ erübrigt sich die Berücksichtigung der Bluglukoseprofile.

B) Anhand des HbA1c lässt sich die Blutglukoseeinstellung während der letzten 2-3 Monate zurückverfolgen.

C) $\mathrm{HbA} 1 \mathrm{c}$ entsteht durch die enzymatische Bindung von Glukose an die endständige $\mathrm{COOH}$ Gruppe der Alphakette des Hämoglobins.

D) HbA1c wird pulsoxymetrisch bestimmt.

E) Der Parameter ist nicht anfällig gegenüber Störfaktoren. 


\subsection{Theoretischer Kurzzeit-Test}

\section{Kasuistik Anhaltender Husten:}

1. Welche Aussage trifft zu?

A) Der THT (Tuberkulinhauttest) erlaubt die Unterscheidung zwischen latenter Infektion und aktiver Erkrankung.

B) Der THT ist als Routinetest bei Gesunden geeignet.

C) Die BCG- Impfung wird heutzutage noch eingeschränkt von der STIKO empfohlen.

D) Bei im Ausland erworbener Tuberkulose besteht keine namentliche Meldepflicht.

E) Bei HIV- positiven Patienten kommt es beim THT zu falsch- negativen Ergebnissen.

2. Welches Antibiotikum gehört nicht zum Standardtherapieschema bei Tuberkulose?

A) Moxifloxacin

B) Isoniazid

C) Rifampicin

D) Pyrazinamid

E) Ethambutol

3. Welche der genannten Maßnahmen kommt am wenigsten in Betracht, wenn auf einer Krankenhausstation bei einem Patienten eine offene Lungentuberkulose diagnostiziert wird?

A) Isolierung des Patienten durch Unterbringung im Einzelzimmer

B) Meldung an das zuständige Gesundheitsamt

C) spezielle Entsorgung der mit infektiösem Material kontaminierten Abfälle

D) umgehende BCG- Impfung bei den Tuberkulin- positiven Kontaktpersonen

E) Röntgenuntersuchung des Thorax bei Kontaktpersonen, bei denen im aktuellen zeitlichen Zusammenhang eine Tuberkulinkonversion stattgefunden hat.

4. Was trifft nicht zur Miliar-Tuberkulose zu?

A) Befällt häufig die Lunge.

B) Nicht selten kommt es zur Hepatosplenomegalie.

C) In der Regel kommt es zu einem isolierten Organbefall.

D) Nebennieren können auch betroffen sein.

E) Es gibt auch eine meningeale Form der Miliar-Tuberkulose

5. Während eines europäischen Kulturfestivals ist eine PJlerin in der Notfallaufnahme eines größeren Krankenhauses tätig. Bei der notfallmäßigen Aufnahmeuntersuchung eines stark betrunkenen Festivalteilnehmers aus Osteuropa hustet dieser die PJlerin mehrmals an. Am Folgetag erfährt diese, dass bei ihm der Verdacht auf eine A)

Tuberkulose besteht. Welche der Aussagen trifft am ehesten zu?

A) Die Inzidenz multiresistenter Tuberkulosebakterien in Osteuropa ist ähnlich wie diejenige in Deutschland.

B) Die Mikroskopie von nach Ziehl-Nielsen gefärbten respiratorischen Materialien weist die höchste diagnostische Sensitivität von Routinemethoden zum Nachweis von Tuberkulosebakterien auf.

C) Gemäß Infektionsschutzgesetz (IfSG) ist bereits der klinische Verdacht auf Tuberkulose nichtnamentlich meldepflichtig (Meldepflicht des behandelnden Arztes).

D) Gemäß Infektionsschutzgesetz (IfSG) ist der Nachweis von Mycobacterium tuberculosis namentlich meldepflichtig (Meldepflicht des Labors).

E) Um bei der PJlerin eine Tuberkulose auszuschließen, sind Serumentnahmen für den Antikörpernachweis direkt nach Exposition sowie drei Wochen später erforderlich.

\section{Kasuistik Dyspnoe und Leistungsminderung:}

1. Ein 5 Monate alter voll gestillter Säugling fällt durch eine blasse Hautfarbe auf. Die Hämoglobinkonzentration im venösen Blut beträgt $91 \mathrm{~g} / \mathrm{l}$. Das MCV beträgt 64 fl. Die Serumferritinkonzentration ist vermindert. Was ist die wahrscheinlichste Ursache der Anämie?
A) B12- Mangel
B) Eisenmangel
C) Folsäuremangel
D) Kugelzellanämie
E) Thalassaemia major

2. Bei einem 15-jährigen Mädchen besteht der Verdacht, dass ihre Anämie auf einem Eisenmangel beruht. Welcher der Blutwerte würde am ehesten den Verdacht einer Eisenmangelanämie stützen?
A) MCV: $64 \mathrm{fl}$
B) Hämatokrit: $49 \%$
C) $\mathrm{MCH}: 35 \mathrm{pg}$

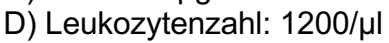
E) Thrombozytenzahl: 350/nl 
3. Eine 45-jährige Patientin mit bekannter rheumatoider Arthritis wird zur Abklärung einer Anämie in einer hämatologischen Klinik vorgestellt. Sie ist seit einigen Monaten weniger leistungsfähig, insbesondere bei körperlicher Belastung. Die Polyarthritis ist derzeit mit einer relativ niedrigen Dosis von Methotrexat eingestellt (10 mg einmal pro Woche). Darunter berichtet die Patientin über wechselnde Arthralgien, die aber deutlich geringer als vor der MTX-Gabe ausgeprägt seien. Klinisch zeigt sich eine blasse Patientin mit geringen Arthritiszeichen - Synovialitis und Überwärmung - im Bereich der Metacarpophalangealgelenke beidseits, sowie im linken Ellenbogengelenk. Welcher Laborwert erlaubt am sichersten die Unterscheidung zwischen einer Eisenmangelanämie und einer sogenannten Anämie der chronischen Erkrankung (Entzündungsanämie)?

A) Serumeisen

B) Haptoglobin

C) Retikulozytenzahl

D) MCV

E) Ferritin

4. Bei einer 44-jährigen, bisher gesunden Verkäuferin wird anlässlich einer Routineuntersuchung ein $\mathrm{Hb}$ Wert von $90 \mathrm{~g} / \mathrm{L}$ gemessen. Die weitere klinisch-chemische Diagnostik ergibt eine erheblich erniedrigte Ferritinkonzentration im Serum und eine erniedrigte Transferrinsättigung. Die Patientin gibt auf Nachfragen an, sich vegetarisch zu ernähren, und berichtet, seit einiger Zeit verstärkte Monatsblutungen zu haben. Der behandelnde Arzt verordnet ein Eisenpräparat zur oralen Anwendung, um den Eisenmangel zu korrigieren. Welche der folgenden Aussagen trifft diesbezüglich am ehesten zu?

A) Zur oralen Therapie eignen sich Eisen-II-Salze.

B) Bei Nüchterneinnahme des Eisenpräparates ist eine bessere Magenverträglichkeit zu erwarten als bei Einnahme zu einer Mahlzeit.

C) Die Bioverfügbarkeit des Eisenpräparates lässt sich durch gleichzeitige Einnahme von Aluminiumphosphat erhöhen.

D) Die Bioverfügbarkeit des Eisenpräparates wird durch gleichzeitige Einnahme von Ascorbinsäure (Vitamin

C) erniedrigt.

E) Im vorliegenden Fall wäre die Gabe von Erythropoetin vor der Eisenbehandlung sinnvoll.

5. Der 2-jährige Emil zeigt eine Entwicklungsverzögerung und eine makrozytäre Anämie. Die 33 Jahre alte Mutter ernährt das Kleinkind rein vegan. Ein Mangel an welchem der folgenden Vitamine könnte am Ehesten Ursache für die erhobenen Befunde sein?
A) Vitamin A
B) Vitamin B6
C) Vitamin B12
D) Vitamin C
E) Vitamin $\mathrm{E}$

\section{Kasuistik Kopfschmerz:}

1. Ein 35-jähriger Kraftfahrer und Kettenraucher wacht - seit 2 Wochen - nachts mit heftigen rechtsseitigen Kopfschmerzen auf, die sich attackenförmig ca. zweistündlich wiederholen und jeweils 30-60 Minuten anhalten. Typische - rechtsseitige - Befunde während der Attacke sind zudem konjunktivale Injektion, Lakrimation, Rhinorrhoe und ein Horner-Syndrom. Welche der folgenden therapeutischen Maßnahmen hat hier die größte Bedeutung?

A) $\mathrm{O}_{2}$-Inhalation

B) Gabe von Glyceroltrinitrat

C) infraorbitale Alkoholinjektion

D) Thermokoagulation des Ganglion stellatum

E) i.v. Infusion von Valproat

2. Was ist bei Migräne am wenigsten zu erwarten?

A) Nasenfluss

B) Aphasie

C) Skotome

D) Lichtscheu

E) Brechreiz

3. Ein 49-jähriger Lehrer leidet seit Jahren mindestens jeden zweiten Tag unter jeweils mehrere Stunden anhaltenden, drückenden, beidseitigen Kopfschmerzen mit bifrontalem Schwerpunkt. Es fühle sich dann etwa so an, als habe er einen Helm auf dem Kopf. Zu Übelkeit oder Erbrechen komme es nicht, auch könne er trotz dieser Kopfschmerzen durchaus weiterarbeiten. Dennoch beeinträchtigten sie seine Lebensqualität erheblich, zumal die üblichen Kopfschmerzmittel wie Acetylsalicylsäure, Paracetamol oder Ibuprofen nicht anschlügen. Er rauche ca. 20 Zigaretten pro Tag, schlafe vermutlich stressbedingt schlecht, sei sonst aber gesund. Der klinisch-neurologische Befund ist inkl. einer Augenhintergrundspiegelung normal, ebenso der 
Blutdruck. Welche der folgenden medikamentösen Therapien kommt für diese Kopfschmerzen am ehesten in Betracht?
A) Acetylsalicylsäure $100 \mathrm{mg} / \mathrm{d}$
B) Amitriptylin $50-150 \mathrm{mg} / \mathrm{d}$
C) Carbamazepin 400-1600 mg/d
D) Indometacin 50-150 mg/d
E) Glukokortikoid-Therapie oral (z.B. Prednisolon-Schema beginnend mit $1 \mathrm{mg} / \mathrm{kg} \mathrm{Körpergewicht)}$

4. Welches der folgenden Medikamente ist für die Akuttherapie einer Migräneattacke nicht geeignet?
A) ASS $1000 \mathrm{mg}$ i.v.
B) MCP $20 \mathrm{mg}$ i.v.
C) Sumatriptan i.n.
D) Propanolol $75 \mathrm{mg}$ p.o.
E) Ibuprofen $600 \mathrm{mg}$ i.v.

5. Welche Symptome können nicht zur Aura einer Migräne gezählt werden?

A) Gesichtsfeldausfälle

B) Konjunktivale Injektion

C) Flimmerskotome

D) Szintillationen

E) Ophthalmoplegie

\section{Kasuistik Kreuzschmerz:}

1. Das Ott-Maß (z.B. Ott 30/34 cm) dient am ehesten zur Beurteilung der...

A) Beugefähigkeit der Brustwirbelsäule

B) Streckfähigkeit der Brustwirbelsäule

C) Streckfähigkeit der Lendenwirbelsäule

D) Streckfähigkeit der Halswirbelsäule

E) Beugefähigkeit der Lendenwirbelsäule

2. Was gehört nicht zu den Red Flags des Kreuzschmerzes?

A) B- Symptomatik

B) Schlafstörungen

C) Blasen- und Mastdarmstörungen

D) Sensibilitätsstörungen der unteren Extremität

E) osteoporotische Wirbelkörperfraktur bei systemischer Steroidtherapie

3. Was siehst du hier im Bild? (Bild eines Tannenbaumphänomens bei Osteoporose)

A) Tannenbaumphänomen bei Skoliose

B) Tannenbaumphänomen bei Osteoporose

C) Wellenzeichen bei Osteoporose

D) Wellenzeichen bei Osteomyelitis

E) Triangle Sign bei Osteomalazie

4. Welche der folgenden Tests und Untersuchungen gehört NICHT zur Untersuchung der Wirbelsäule?

A) Finger- Boden- Abstand

B) Lasègue- Zeichen

C) Inspektion der Kyphosen und Lordosen

D) Varus- und Valgus Stresstest

E) Palpation der Dornfortsätze

5. Eine 63-jährige Patientin leidet an chronischen Rückenschmerzen, die durch eine Osteoporose bedingt sind. Welche der Kombinationen physikalisch-therapeutischer Maßnahmen ist am sinnvollsten?
A) Eispackungen und tonisierende Klopfungen am Rücken
B) örtliche Wärmeanwendungen und Kräftigung der Rückenmuskulatur
C) Unterwasserhochdruckstrahlmassagen und chirotherapeutische Manipulationen der Wirbelsäule
D) Suspension im Schlingenbett und Traktion der Wirbelsäule
E) Bindegewebsmassagen und kalte Blitzgüsse

\section{Kasuistik Polyurie:}

1. Was gehört nicht zum sogenannten Metabolischen Syndrom?

A) arterielle Hypertonie

B) Typ- 2 Diabetes mellitus

C) erhöhte Triglyzeride (>150 mg/dl)

D) Nüchternblutzucker $>100 \mathrm{mg} / \mathrm{dl}$

E) niedriges LDL- Cholesterin $(<50 \mathrm{mg} / \mathrm{dl})$ 
2. Ein 56-jähriger Patient wird seit 30 Jahren wegen Typ-1-Diabetes mit Insulin (intensiviertes Therapieschema) behandelt. Er wurde im letzten Monat zweimal wegen schwerer Hypoglykämie notärztlich versorgt. Seine Ehefrau gibt an, dass er, anders als früher, die Hypoglykämien nicht bemerkt habe und keine Gegenmaßnahmen habe treffen können. Was könnte am Ehesten der Grund für die verminderte Eigenwahrnehmung der Hypoglykämien sein?

A) Neuropathie mit Beteiligung des autonomen Nervensystems

B) besonders schnelle Absenkung des Blutzuckers

C) Wachstumshormon- Überproduktion (Akromegalie)

D) Nebennierenüberfunktion

E) Hyperthyreodismus

3. Bei welchem Antidiabetikum besteht am ehesten die Gefahr einer Laktatazidose?

A) Acarbose

B) Metformin

C) Insulin lispro

D) Glibenclamid

E) Repaglinid

4. Eine 65- jährige Frau mit einem seit 3 Jahren bekannten Diabetes mellitus Typ 2 sucht Sie erstmals auf. Welcher der Nachweise ist am besten geeignet, nach dem ersten Zeichen einer diabetischen Nephropathie zu suchen?
A) Glukosurie (Stix)
B) Serumkreatinin-Konzentration
C) Mikroalbuminurie (Stix oder Elisa)
D) Mikrohämaturie (Stix)
E) Eine Suche ist nicht notwendig, da nach 3- jähriger Diabetesdauer noch keine Nephropathie vorliegt.

5. Ab welchem Nüchternblutzuckerwert (8h Nahrungskarenz) spricht man von einem Diabetes mellitus?

A) $116 \mathrm{mg} / \mathrm{dl}$

B) $126 \mathrm{mg} / \mathrm{dl}$

C) $150 \mathrm{mg} / \mathrm{dl}$

D) $200 \mathrm{mg} / \mathrm{dl}$

E) $210 \mathrm{mg} / \mathrm{dl}$

Kasuistik Schwangerschaft:

1. Die häufigste Ursache einer Schwangerschaftsanämie mit Hämoglobinwerten unter $110 \mathrm{~g} / \mathrm{l}$ ist:

A) ein Eisenmangel

B) ein latenter Folsäuremangel

C) ein Vitamin-B-12- Mangel

D) ein erhöhter Folsäurebedarf des Fetus

E) eine verminderte Eisenresorption im Darm

2. Die Geburt eines Kindes, hier mal Peter genannt, erfolgte am errechneten Geburtstermin. Berechnen Sie diesen gemäß der Naegele-Regel unter Zugrundelegung folgender Angaben: In welchem Zeitraum war bei Annahme eines ungestörten Schwangerschaftsverlaufes - Peters Geburt vorrangig zu erwarten?

Zykluslänge: 28 Tage; 1. Tag der letzten Regelblutung: 5. März; Konzeption: 18. März
A) 10.-12. November
B) 24.-26. November
C) 1.-3. Dezember
D) 10.-12. Dezember
E) 24.-26. Dezember

3. Welche der folgenden Symptome passt nicht zum Bild einer Präeklampsie:
A) Oligurie
B) Proteinurie
C) Hypertonie
D) starke Gewichtsabnahme
E) ggf. Ödeme

4. Welche Serologischen Untersuchungen werden bei schwangeren Frauen NICHT häufig durchgeführt bzw. angeboten?
A) Lues Suchtest (TPHA)
B) HIV-AK
C) HSV-2 PCR
D) CMV-AK
E) $\mathrm{HBs} A \mathrm{G}$ 
5. Nach Bestätigung der Schwangerschaft führt der Frauenarzt ein ausführliches Beratungsgespräch zur Schwangerschaft mit Frau B., bei dem auch die Ernährung zur Sprache kommt. Was empfiehlt der Frauenarzt seiner Patientin am Ehesten?

A) Verzicht auf Rohmilchprodukte, auch auf Weichkäse aus Rohmilch wegen der Infektionsgefahr

B) Reduzierung von Milch und milchhaltigen Produkten insgesamt wegen des hohen Kalziumgehaltes

C) ballaststoffarme Ernährung wegen der verminderten Darmmotilität

D) allgemeine Steigerung der Nahrungszufuhr, vor allem im letzten Trimenon um ca. $1500 \mathrm{kcal} / \mathrm{Tag}$ wegen des erhöhten Bedarfs

E) eiweißarme Mischkost zur Reduzierung des Gestoserisikos

\section{Kasuistik arterielle Hypertonie:}

1. Bei welchem Blutdruck spricht man von Hypertensiver Krise/ Notfall?
A) $200 / 100 \mathrm{mmHg}$
B) $210 / 110 \mathrm{mmHg}$
C) $220 / 120 \mathrm{mmHg}$
D) $230 / 130 \mathrm{mmHg}$
E) $240 / 140 \mathrm{mmHg}$

2. Was gehört nicht zu den typischen Risikofaktoren einer primären Hypertonie?

A) Adipositas

B) Nikotinabusus

C) Diabetes mellitus

D) Hypercholesterinämie

E) erhöhter Vitamin- D Spiegel

3. Was gehört NICHT zu den Ursachen eines sekundären Hypertonus?

A) pAVK (periphere arterielle Verschlusskrankheit)

B) Schwangerschaft

C) Nierenarterienstenose

D) Conn-Syndrom

E) endokrine Hypertonie

4. Eine 62-jährige Patientin mit primärer arterieller Hypertonie hat unter Behandlung mit Hydrochlorothiazid eine unzureichende Blutdrucksenkung gezeigt. Es wird jetzt eine leichte Linksherzinsuffizienz festgestellt. Welche der folgenden Maßnahmen ist als nächste - unter Beachtung der Richtlinien für eine vorsichtige Therapieumstellung - am ehesten zu empfehlen?

A) Ersatz des Hydrochlorothiazids durch Dihydralazin

B) Ersatz des Hydrochlorothiazids durch ein Reserpinhaltiges Antihypertensivum

C) zusätzliche Behandlung mit Furosemid

D) zusätzliche Behandlung mit Doxazosin

E) zusätzliche Behandlung mit Enalapril

5. Bei einem 55-jährigen Patienten wird die Diagnose einer sekundären Hypertonie bei Nierenarterienstenose gestellt. Was kommt als Ursache dieser hochdruckwirksamen Nierenarterieneinengung vorrangig in Betracht?
A) fibromuskuläre Dysplasie
B) Nierenarterienaneurysma
C) Arteriosklerose
D) Nierenarterienhypoplasie
E) Kompression durch benachbarten raumfordernden Prozess 


\subsection{Beispiel Bewertungsbogen im Wahlfach OSCE}

Station $1 \quad$ Herzuntersuchung

OSCE Studentische Poliklinik

Nummer des Studenten:

Zu beurteilen ist die Untersuchungstechnik während der körperlichen Untersuchung bei Verdacht auf eine koronare Herzerkrankung.

Block A

Checkliste körperliche Untersuchung:

Inspektion allgemein

nicht versucht falsch richtig

(0)

(1) (2)

- Allgemeinzustand, Ernährungszustand (normal, adipös) (1/2=1 Pkt., 2/2=2 Pkt.)

- Augen (keine Xanthelasmen oder Arcus lipoides...)

$[$ ] ]............. [ ] .......... [ ]

- Jugularvenen (nicht gestaut)

- Hände (keine Trommelschlegelfinger) $[$ [ ] .............. [ ] .......... [ ]

- Beine (keine Ödeme)

Auskultation Karotiden (unauffällig) []$\ldots \ldots \ldots \ldots . . . . . . . . . . .[]$

Palpation Herzspitzenstoß (5. ICR links MCL)

[ ] ]............. [ ] ......... [ ]

Auskultation Herz

- 2. ICR rechts parasternal, 2. ICR links parasternal,

3. ICR links parasternal, 4. ICR rechts parasternal,

5. ICR links $\mathrm{MCL}$

(HAT rein, kein Strömungsgeräusch)

(2/5=1 Pkt., 4/5=2 Pkt.)

Palpation arterielle Pulse

- A. radialis bds., A. carotis bds., A. femoralis bds. $[$ ] ]............ [ ] ......... [ ]

A. poplitea bds., A. tibialis post. bds.,

A. dorsalis pedis bds. (unauffällig)

(2/6=1 Pkt., 4/6=2 Pkt.)

Block B

Schwerpunkt Untersuchungstechnik und Umgang mit dem Patienten

schlecht mittelmäßig gut

- Optimale Bedingungen

(0)

(1)

(2)

- Sinnvolle Reihenfolge

.. [ ] [............... [ ] [............ [ ]

- Anrede, Vorstellung, Freundlichkeit, Rücksichtnahme

- Sicheres Auftreten

Auswertung:

Bock A _ von 18 Pkt. (entspricht 70\%)

Block B_ von 8 Pkt. (entspricht $30 \%$ )

Gesamtprozentzahl: 


\subsection{Fragebogen Selbsteinschätzung}

1. Eine aktuelle Anamnese erheben können und relevante Daten aus der Vorgeschichte (Vorerkrankungen, Medikamente, Risikofaktoren) erfragen

\begin{tabular}{l|l|l|l|l|l|l|l} 
sehr sicher & 1 & 2 & 3 & 4 & 5 & 6 & überhaupt nicht sicher \\
\hline
\end{tabular}

2. Die korrekte Diagnostik bei häufigen Beratungsanlässen auswählen und durchführen

\begin{tabular}{|c|l|l|l|l|l|l|l|}
\hline sehr sicher & 1 & 2 & 3 & 4 & 5 & 6 & überhaupt nicht sicher \\
\hline
\end{tabular}

3. Eine Diätberatung bei gegebenem Anlass (Hyperlipidämie, Hyperurikämie...) durchführen

\begin{tabular}{|c|l|l|l|l|l|l|l|}
\hline sehr sicher & 1 & 2 & 3 & 4 & 5 & 6 & überhaupt nicht sicher \\
\hline
\end{tabular}

4. Eine Krebsvorsorgeuntersuchung durchführen (Rektale Untersuchung, Palpation der Brust, Anleitung zur Palpation...)

\begin{tabular}{l|l|l|l|l|l|l|l} 
sehr sicher & 1 & 2 & 3 & 4 & 5 & 6 & überhaupt nicht sicher \\
\hline
\end{tabular}

5. Eine Impfberatung durchführen

\begin{tabular}{|c|l|l|l|l|l|l|l|}
\hline sehr sicher & 1 & 2 & 3 & 4 & 5 & 6 & überhaupt nicht sicher \\
\hline
\end{tabular}

6. Im Gespräch angemessen auf den Patienten einzugehen
\begin{tabular}{|c|c|c|c|c|c|c|c|}
\hline sehr sicher & 1 & 2 & 3 & 4 & 5 & 6 & überhaupt nicht sicher
\end{tabular}

7. Überbringen schlechter Nachrichten

\begin{tabular}{|c|l|l|l|l|l|l|l|}
\hline sehr sicher & 1 & 2 & 3 & 4 & 5 & 6 & überhaupt nicht sicher \\
\hline
\end{tabular}

8. Emotionale und psychische Ursachen für Erkrankungen erkennen

\begin{tabular}{|c|l|l|l|l|l|l|l|}
\hline sehr sicher & 1 & 2 & 3 & 4 & 5 & 6 & überhaupt nicht sicher \\
\hline
\end{tabular}

9. Untersuchung des Herzkreislaufsystems

\begin{tabular}{|c|l|l|l|l|l|l|l|}
\hline sehr sicher & 1 & 2 & 3 & 4 & 5 & 6 & überhaupt nicht sicher \\
\hline
\end{tabular}

10. Untersuchung des Abdomens inkl. Rektale Untersuchung

\begin{tabular}{|c|l|l|l|l|l|l|l|}
\hline sehr sicher & 1 & 2 & 3 & 4 & 5 & 6 & überhaupt nicht sicher \\
\hline
\end{tabular}

11. Untersuchung von Venen und Arterien

11. Untersuchung von Venen und Arterien
\begin{tabular}{|c|c|c|c|c|c|c|c|}
\hline sehr sicher & 1 & 2 & 3 & 4 & 5 & 6 & überhaupt nicht sicher \\
\hline
\end{tabular}

12. Untersuchung der Lunge

\begin{tabular}{|c|l|l|l|l|l|l|l|}
\hline sehr sicher & 1 & 2 & 3 & 4 & 5 & 6 & überhaupt nicht sicher \\
\hline
\end{tabular}

13. Untersuchung der Gelenke und Wirbelsäule

13. Untersuchung der Gelenke und Wirbelsäule
\begin{tabular}{|c|c|c|c|c|c|c|c|}
\hline sehr sicher & 1 & 2 & 3 & 4 & 5 & 6 & überhaupt nicht sicher \\
\hline
\end{tabular}

14. Untersuchung lymphatische Organe (Lymphknoten und Milz)

\begin{tabular}{|c|l|l|l|l|l|l|l|}
\hline sehr sicher & 1 & 2 & 3 & 4 & 5 & 6 & überhaupt nicht sicher \\
\hline
\end{tabular}

15. Untersuchung der Haut

15. Untersuchung der Haut
\begin{tabular}{|c|c|c|c|c|c|c|c|}
\hline sehr sicher & 1 & 2 & 3 & 4 & 5 & 6 & überhaupt nicht sicher \\
\hline
\end{tabular}

16. Neurologische Untersuchung (Hirnnerven, Pupillenreflex, Reflexe)

\begin{tabular}{|c|c|c|c|c|c|c|c|}
\hline sehr sicher & 1 & 2 & 3 & 4 & 5 & 6 & überhaupt nicht sicher \\
\hline 17. Untersuchung von Auge, Nase, Rachen und Ohr \\
\begin{tabular}{|l|c|c|c|c|c|c|c|}
\hline sehr sicher & 1 & 2 & 3 & 4 & 5 & 6 & überhaupt nicht sicher \\
\hline
\end{tabular}
\end{tabular}


18. EKG (Durchführung und Befundung)
\begin{tabular}{|c|c|c|c|c|c|c|c|}
\hline sehr sicher & 1 & 2 & 3 & 4 & 5 & 6 & überhaupt nicht sicher \\
\hline
\end{tabular}

19. Blutdruckmessung

\begin{tabular}{|c|l|l|l|l|l|l|l|}
\hline sehr sicher & 1 & 2 & 3 & 4 & 5 & 6 & überhaupt nicht sicher \\
\hline
\end{tabular}

20. Sonographie Abdomen (Durchführung und Befundung)

\begin{tabular}{|c|c|c|c|c|c|c|c|}
\hline sehr sicher & 1 & 2 & 3 & 4 & 5 & 6 & überhaupt nicht sicher \\
\hline
\end{tabular}

\section{Hygienisches Arbeiten}

\begin{tabular}{|c|l|l|l|l|l|l|l|}
\hline sehr sicher & 1 & 2 & 3 & 4 & 5 & 6 & überhaupt nicht sicher \\
\hline
\end{tabular}

22. Legen eines Venenverweilkatheters

\begin{tabular}{|c|c|c|c|c|c|c|c|}
\hline sehr sicher & 1 & 2 & 3 & 4 & 5 & 6 & überhaupt nicht sicher \\
\hline
\end{tabular}

23. Urinstix (Durchführen, Indikation zur weiteren Diagnostik)

\begin{tabular}{|c|l|l|l|l|l|l|l|}
\hline sehr sicher & 1 & 2 & 3 & 4 & 5 & 6 & überhaupt nicht sicher \\
\hline
\end{tabular}

24. I.m. Injektionen und Impfungen

\begin{tabular}{|c|c|c|c|c|c|c|c|}
\hline sehr sicher & 1 & 2 & 3 & 4 & 5 & 6 & überhaupt nicht sicher \\
\hline
\end{tabular}

25. Erhobene Laborwerte in Zusammenschau mit der Krankengeschichte eines Patienten auswerten

\begin{tabular}{|c|l|l|l|l|l|l|l|}
\hline sehr sicher & 1 & 2 & 3 & 4 & 5 & 6 & überhaupt nicht sicher \\
\hline
\end{tabular}

26. Einen Therapieplan für einen Patienten erstellen

26. Einen Therapieplan für einen Patienten erstellen
\begin{tabular}{|c|l|l|l|l|l|l|l|}
\hline sehr sicher & 1 & 2 & 3 & 4 & 5 & 6 & überhaupt nicht sicher \\
\hline
\end{tabular}

27. Einen Bluthochdruck leitliniengerecht medikamentös einstellen

\begin{tabular}{|c|l|l|l|l|l|l|l|}
\hline sehr sicher & 1 & 2 & 3 & 4 & 5 & 6 & überhaupt nicht sicher \\
\hline
\end{tabular}

28. Differentialdiagnosen gegeneinander abwägen

\begin{tabular}{|c|c|c|c|c|c|c|c|}
\hline 28. Differentialdiagnosen gegeneinander abwägen \\
\hline sehr sicher & 1 & 2 & 3 & 4 & 5 & 6 & überhaupt nicht sicher \\
\hline
\end{tabular}

29. Eine Patientenversorgung organisieren (Einbeziehen von anderen Berufsgruppen wie Physiotherapeuten oder Hebammen)

\begin{tabular}{|c|l|l|l|l|l|l|l|}
\hline sehr sicher & 1 & 2 & 3 & 4 & 5 & 6 & überhaupt nicht sicher \\
\hline
\end{tabular}

30. Einen Diabetes mellitus leitliniengerecht einstellen

30. Einen Diabetes mellitus leitliniengerecht einstellen
\begin{tabular}{|c|l|l|l|l|l|l|l|}
\hline sehr sicher & 1 & 2 & 3 & 4 & 5 & 6 & überhaupt nicht sicher
\end{tabular}

31. Eine Routine- Schwangerschaftsuntersuchung durchführen

\begin{tabular}{|c|l|l|l|l|l|l|l|}
\hline sehr sicher & 1 & 2 & 3 & 4 & 5 & 6 & überhaupt nicht sicher \\
\hline
\end{tabular}

32. Einen Mutterpass ausstellen

32. Einen Mutterpass ausstellen
\begin{tabular}{|c|c|c|c|c|c|c|c|}
\hline sehr sicher & 1 & 2 & 3 & 4 & 5 & 6 & überhaupt nicht sicher \\
\hline
\end{tabular}

33. Kreuzschmerzen leitliniengerecht therapieren

33. Kreuzschmerzen leitliniengerecht therapieren
\begin{tabular}{|c|l|l|l|l|l|l|l|}
\hline sehr sicher & 1 & 2 & 3 & 4 & 5 & 6 & überhaupt nicht sicher \\
\hline
\end{tabular}

34. Umgang mit Tuberkulose- Patienten (Diagnostik, Therapie, ...)

\begin{tabular}{|c|c|c|c|c|c|c|c|}
\hline sehr sicher & 1 & 2 & 3 & 4 & 5 & 6 & überhaupt nicht sicher \\
\hline
\end{tabular}




\section{DANKSAGUNG}

An dieser Stelle bedanke ich mich herzlichst bei meinem Doktorvater Prof. Dr.

Dr. Dr. Robert Sader für die Überlassung dieses innovativen und interessanten Themas.

Mein besonderer Dank gilt meinem Betreuer Dr. Lukas Seifert für die Konzeption und Fragestellung dieser Arbeit. Ich danke inm für die stetige Hilfsbereitschaft, Geduld und konstruktive Kritik. Durch seine fachlichen Hinweise und das mühevolle Korrekturlesen hat er wesentlich zum erfolgreichen Abschluss dieser Arbeit beigetragen.

Anschließend danke ich meinen Eltern, Großeltern und meinem Bruder, die mir mein Studium ermöglichten und mir stets unterstützend und liebevoll zur Seite standen. Ihnen ist die Arbeit gewidmet. 